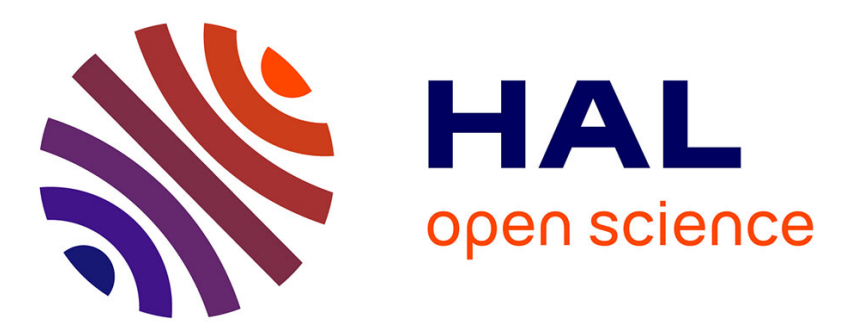

\title{
Low order modeling of vortex driven self-sustained pressure pulsations in solid rocket motors \\ Lionel Hirschberg
}

\section{To cite this version:}

Lionel Hirschberg. Low order modeling of vortex driven self-sustained pressure pulsations in solid rocket motors. Other. Université Paris Saclay (COmUE), 2019. English. NNT : 2019SACLC001 . tel-02019520

\section{HAL Id: tel-02019520 \\ https://theses.hal.science/tel-02019520}

Submitted on 14 Feb 2019

HAL is a multi-disciplinary open access archive for the deposit and dissemination of scientific research documents, whether they are published or not. The documents may come from teaching and research institutions in France or abroad, or from public or private research centers.
L'archive ouverte pluridisciplinaire HAL, est destinée au dépôt et à la diffusion de documents scientifiques de niveau recherche, publiés ou non, émanant des établissements d'enseignement et de recherche français ou étrangers, des laboratoires publics ou privés. 
unıversitè PARIS-SACLAY

\section{Low order modeling of vortex driven self-sustained pressure pulsations in solid rocket motors}

Thèse de doctorat de l'Université Paris-Saclay préparée à CentraleSupélec

Ecole doctorale n`579 Sciences mécaniques et énergétiques, matériaux et géosciences (SMEMaG)

Spécialité de doctorat: Mécanique des Fluides

Thèse présentée et soutenue à Gif-sur-Yvette, le 16 Janvier 2019, par

M. LiONEL HIRSCHBERG

Composition du Jury :

Michel Roger

Professeur, École Centrale de Lyon

Maria Heckl

Professeur, Keele University

Yves Aurégan

Directeur de recherche CNRS, LAUM

Jérôme Anthoine

HDR, ONERA

Steven J. Hulshoff

Professeur assistant, TU Delft

Thierry Schuller

Professeur, IMFT

Christophe Schram

Professeur, VKI

Jean Collinet

Ingénieur, ArianeGroup
Président

Rapporteur

Rapporteur

Examinateur

Examinateur

Directeur de thèse

Co-encadrent

Co-encadrent 



\section{Remerciements}

Thanks is due to my supervisors Ir. Jean Collinet, Prof. Christophe Schram and Prof. Thierry Schuller for their support. Thanks to Dr. Jérôme Anthoine, Dr. Yves Aurégan, Prof. Maria Heckl, Dr. Steven J. Hulshoff, and Prof. Michel Roger for participating as jury members.

Special thanks is due to Dr. Steven J. Hulshoff, who has contributed to this work in unmeasurable ways. Thanks for an efficient and fruitful collaboration.

To Serge Radulovic thank you for your support.

Thanks to Luca Minelli \& folks, Ruben Di Battista, Marta \& Theo Driessen, Andrea Scagliarini \& folks, Kim, Maurice, José \& Herman Fransen, Floor \& Jeroen van Beeck, Stefania \& Daniel Guariglia, Majdouline Borij, Yehuda Dinaii, Marcel van den Berg, Arya Ranucci, Massimo Ranucci, Shoko, Pascal Durrieu, Roger Jeurissen, Lior Geffen, Elena Grof \& Alessandro Zarri, Riccardo Zamponi, Jenny \& John Harper, Enrique Guzman-Vazquez, Coriandre Vilain, Nikita Allgire, Beatrix Barroso, P. T., Sylvie Dequand, Maud Leroux, your friendship is profoundly appreciated.

Thanks to the EM2C support staff: Noï Lavaud, Natalie Rodriquez and Brigitte llobel and Sébastien Turgis. I thank Evelyne Crochard, Christelle Debeer, Raimondo Giammanco, the members of the HPCC \& IT Service and secretariat of the VKI for their help as well as Catherine Lhopital and Nathalie Cornay of SMEMaG.

Thanks to my comrades in arms of the EM2C lab: Aurélien Genot, Robin Nez, Valentin Dupif, Claire Beauchesne, Arnaud Galland, Milan Pelletier, David Marchal, Gilles Bailet and Kevin Torres-Monclard.

Last but not least my close and extended family. I am grateful for your love and support, which undoubtedly canalized this accomplishment.

This work is dedicated to the memory of my grandmother Lydia HirschbergNémirovsky (RIP June 25th 2018). 


\section{Abstract}

Solid Rocket Motors (SRMs) can display self-sustained acoustic oscillations driven by coupling between hydrodynamic instabilities of the internal flow and longitudinal acoustic standing waves. The hydrodynamic instabilities are triggered by the acoustic standing wave and results in the formation of coherent vortical structures. For nominal ranges of flow conditions the sound waves generated by the interaction between these vortices and the choked nozzle at the end of the combustion chamber reinforces the acoustic oscillation. Most available literature on this subject focuses on the threshold of instability using a linear model. The focus of this work is on the prediction of the limit-cycle amplitude. The limit cycle is reached due to nonlinear saturation of the source, as a consequence of the formation of large coherent vortical structures. In this case the vortex-nozzle interaction becomes insensitive to the amplitude of the acoustic standing wave. Hence, one can focus on the sound generation of a vortex with the nozzle. Sound production can be predicted from an analytical two-dimensional planar incompressible frictionless model using the so-called Vortex Sound Theory. In this model the vorticity is assumed to be concentrated in a line vortex. Experiments indicate that the volume of cavities around so-called "integrated nozzles" have a large influence on the pulsation amplitude for large SRMs. This is due to the acoustical field normal to the vortex trajectory, induced by the compressibility of the gas in this cavity. As an alternative to the incompressible analytical model a compressible frictionless model with an internal Euler Aeroacoustic (EIA) flow solver is used for simulations of vortex-nozzle interaction. A dedicated numerical simulation study focusing on elementary processes such as vortex-nozzle and entropy spot-nozzle interaction allows a systematic variation of relevant parameters and yields insight which would be difficult by means of limit cycle studies of the full engine. A systematic study of the vortex-nozzle interaction in the case of a choked nozzle has been undertaken by varying: the upstream Mach number, the vortex-core radius, the upstream point where the vortex is released and the vortex circulation. The results are summarized by using a lumped element model for plane wave propagation, which is based on theoretical scaling laws. This model provides physical insight and predicts the acoustic pulse amplitude due to vortex nozzle-interaction within $25 \%$. Simulations are made for integrated nozzles with a surrounding cavity and nozzle inlets mounted flush in a wall normal to the 
combustion chamber wall. The Euler code is also used to explore the potential use of nozzle with a gradual ramp as an inlet. Such a nozzle could reduce the limit-cycle pulsation amplitude. From EIA simulations it appears that sound due to vortex-nozzle interaction is mainly generated during the approach phase and that for the relevant parameter range there is no impingement of the vortex on the nozzle wall as has been suggested in the literature. In the incompressible analytical model the effect of the nozzle cavity is represented by a fluctuating volume line source. Using an energy balance approach, a single fit-parameter model is formulated which qualitatively predicts limit-cycle observations in cold gas-scale experiments reported in the literature. Finally the Euler model is used to compare the sound production by vortex-nozzle interaction with that due to the ingestion of an entropy non-uniformity also called entropy spot. In addition to insight, this study provides a systematic procedure to develop a lumped element model for the sound source due to non-homogeneous flow-nozzle interactions in SRMs. Such lumped models based on experimental data or a limited number of flow simulations can be used to ease the design of SRMs. 


\section{Contents}

Abstract

Nomenclature $\quad$ Xv

1 Introduction $\quad 1$

1.1 Large solid rocket motors . . . . . . . . . . . . . . . 1

1.2 Statement of the problem . . . . . . . . . . . . 4

1.3 Focus of the study . . . . . . . . . . . . . . . . . . . 9

1.4 Literature review . . . . . . . . . . . . . . . . . . . . 10

1.5 Research objectives . . . . . . . . . . . . . . . . 21

1.6 Thesis overview . . . . . . . . . . . . . . . . . 23

2 Global lumped element model for vortex sound in large Solid Rocket Motors $\quad 25$

2.1 Vortex sound analogy . . . . . . . . . . . . . 25

2.2 Low Mach number approximation derivation of the pulsation amplitude harmonic oscillator equation. . . . . . . . . . . . 29

2.3 Conclusion . . . . . . . . . . . . . . . . . . . . 34

3 Analytical incompressible frictionless two-dimensional planar model for vortex-nozzle interaction $\quad 35$

3.1 Introduction . . . . . . . . . . . . . . . . . . 36

3.2 Potential theory . . . . . . . . . . . . . . . . . 37

3.3 Schwartz-Christoffel transformation . . . . . . . . . . . . 38

3.4 Vortex path . . . . . . . . . . . . . . . . 41

3.5 Vortex sound theory: Howe's approximation . . . . . . . . . . 42

3.6 Sound generation . . . . . . . . . . . . . . . . . . . . . 42

3.7 Results. . . . . . . . . . . . . . . . . . . . 46

3.8 Conclusions . . . . . . . . . . . . . . . . . . . . 52

4 Vortex-nozzle interaction: two-dimensional plane Euler simulations based lumped element sound source model 53

4.1 Introduction . . . . . . . . . . . . . . . . . . 54

4.2 Relevant parameter ranges . . . . . . . . . . . . 56 
4.3 Nozzle inlet geometries . . . . . . . . . . . . . . . . . . 58

4.4 Sound production and scaling rules . . . . . . . . . . . . 60

4.5 Lumped element model . . . . . . . . . . . . . . . . . . . 62

4.6 Euler two-dimensional plane model . . . . . . . . . . . . . 68

4.7 Parametric study of vortex-nozzle interaction with nozzle cavity 78

4.8 Parametric study of vortex-nozzle interaction for the right angle nozzle inlet without cavity . . . . . . . . . . . . . 84

4.9 Effective volume $\tilde{V}_{\text {eff }} \ldots \ldots \ldots \ldots$

4.10 Fit of data using lumped element model . . . . . . . . . . . . . . 93

4.11 Response of a gradual ramp inlet nozzle . . . . . . . . . . . . 99

4.12 Conclusion . . . . . . . . . . . . . . . . . . . 105

5 Analytical energy balance prediction of limit-cycle amplitude 109

5.1 Introduction . . . . . . . . . . . . . . . . . . . 110

5.2 Cold gas model with axial injection . . . . . . . . . . . . 113

5.3 Theoretical analysis . . . . . . . . . . . . . . . . . . 118

5.4 Results . . . . . . . . . . . . . . . . . . . . . . 126

5.5 Conclusion . . . . . . . . . . . . . . . . . . . . . . . 134

6 Entropy indirect noise compared to vortex driven indirect noise in large solid rocket motors: a numerical investigation of the $\begin{array}{ll}\text { influence of a nozzle cavity } & 137\end{array}$

6.1 Introduction . . . . . . . . . . . . . . . . . . . . 138

6.2 Description of the computational approach . . . . . . . . . . 139

6.3 Influence of upstream Mach number on upstream acoustic response140

6.4 Influence of core radius on upstream acoustic response . . . . . 142

6.5 Influence of release height on upstream acoustic response . . . . 144

6.6 Comparison of the effect of a nozzle cavity volume on vortexnozzle and entropy-spot-nozzle induced indirect noise . . . . . . 146

6.7 Conclusion . . . . . . . . . . . . . . . . . . . . . . . . . . 148

$\begin{array}{ll}\text { Conclusion } & 151\end{array}$

$\begin{array}{ll}\text { A Orthogonality condition } & 159\end{array}$

B Details derivation harmonic oscillator model 161

C Ratio of volume integral to surface integral 165

$\begin{array}{ll}\text { D Schwartz-Christoffel transformation } & 167\end{array}$

E Vortex path in two-dimensional incompressible flow theory 173

F Derivation of equation used to determine the order of accuracy 179

$\begin{array}{lll}\text { G } & \text { Vortex passing through a nozzle with cavity } & 181\end{array}$ 
H Acoustic loss models 183

H.1 Nozzle radiation . . . . . . . . . . . . . . . . . . . . . . 183

H.2 Vortex shedding at the orifice . . . . . . . . . . . . . . 184

H.3 Viscous damping at the porous wall . . . . . . . . . . . 185

I Euler Internal Aeroacoustics code 187

I.1 Euler equations . . . . . . . . . . . . . . . . . . . . 187

I.2 Advantages and disadvantages applying Euler equations . . . . 188

I.3 Boundary conditions . . . . . . . . . . . . . . . . . . . . . . . . 189

I.4 Numerical techniques . . . . . . . . . . . . . . . . . . . . . . . 191

$\begin{array}{lll}\text { J Entropy spot generation } & 199\end{array}$

K List of publications $\quad 205$

K.1 Peer reviewed conference proceedings . . . . . . . . . . . . 205

K.2 Peer reviewed published journal articles . . . . . . . . . 205

K.3 Manuscript submitted for peer review . . . . . . . . . 206

K.4 Manuscript being prepared . . . . . . . . . . . . . 206

L Résumé en Français $\quad 207$

$\begin{array}{ll}\text { References } & 217\end{array}$ 



\section{List of Tables}

5.1 Nozzle numbers and volumes . . . . . . . . . . . . . . . 126

5.2 Overview of the results of experiments, with axial injection obtained with the cold flow scale model of the VKI . . . . . . . 128

5.3 Influence of cavity volume $V_{c} \ldots \ldots \ldots \ldots \ldots \ldots$

5.4 Influence of pipe length $L_{p} \ldots \ldots \ldots \ldots \ldots \ldots$

5.5 Influence of the distance between the orifice and nozzle inlet $L_{o} \quad 132$

5.6 Influence of orifice radius $r_{o}, L_{o}=7.1 \times 10^{-2} \mathrm{~m} \ldots \ldots \ldots 133$

5.7 Influence of orifice radius $r_{o}, L_{o}=4.6 \times 10^{-2} \mathrm{~m} \ldots \ldots \ldots$ 



\section{List of Figures}

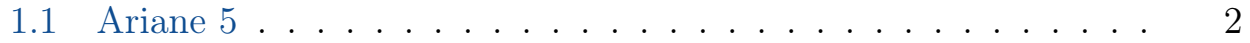

1.2 Ariane 5 P230 SRM . . . . . . . . . . . . . . . 2

1.3 Star-shaped S1 propellant segment of a P230 . . . . . . . . 3

1.4 Nozzle during launch . . . . . . . . . . . . . . . . . . . . 3

1.5 Feedback loop in self-excited systems. . . . . . . . . . . 4

1.6 St as a function of $R e$ smooth cylinder . . . . . . . . . . 7

1.7 Cold gas scale model sketch . . . . . . . . . . . . . 7

1.8 High amplitude pulsations in a small SRM . . . . . . . . . . . 11

1.9 Types of vortex shedding . . . . . . . . . . . . . 13

1.10 Frequency signature Titan $4 \ldots \ldots \ldots . \ldots . \ldots . \ldots 14$

1.11 Frequency signature Ariane 5 P230 . . . . . . . . . . . 15

1.12 OVS simulation LP3 motor . . . . . . . . . . . . . . 15

1.13 Taylor-Culick flow . . . . . . . . . . . . . . . . 18

1.14 Comparison of complex frequency $\omega=\omega_{r}+i \omega_{i}$ to LP9 scalemodel data. . . . . . . . . . . . . . . . . . . . 19

3.1 Smooth contraction geometry . . . . . . . . . . . . . . 40

3.2 Vortex path analytical model vortex nozzle interaction . . . . . 47

3.3 Pulse in total enthalpy $B^{\prime}$ generated by the vortex $(|\tilde{\Gamma}|=0.8)$ interaction with the contraction. . . . . . . . . 48

3.4 Evolution of the coordinates of the vortex $(|\tilde{\Gamma}|=0.8)$ position $\left(x_{\Gamma}, y_{\Gamma}\right)$ as a function of time. . . . . . . . . . 48

3.5 Analytical model acoustic response sharp contraction . . . . . . 49

3.6 Total enthalpy pulse calculated with analytical model . . . . . . 49

3.7 Analytical vortex nozzle interaction model: influence of circulation 50

3.8 Analytical vortex nozzle interaction model: influence of circulation scaled results . . . . . . . . . . . . . . 51

3.9 Influence of width $d / S_{1}$ of contraction (curvature of edge) sound pulse generated by the vortex with dimensionless circulation $|\tilde{\Gamma}|=0.8$ released at height $h / S_{1}=0.4$ for a contraction $S_{1} / S_{2}=3.51$

3.10 Influence of the circulation $d / S_{1}$ on the dimensionless time integral of the acoustical pulse $\frac{2 S_{1} U \int B^{\prime} \mathrm{d} t}{\Gamma^{2}}$ for a contraction $S_{1} / S_{2}=352$ 
4.1 Laminar radial injection simulation for $M=0.09$ taken from (Anthoine 2000) . . . . . . . . . . . . . . . 57

4.2 Nozzle inlet geometries studied . . . . . . . . . . . . . . . 58

4.3 Relation cylindrical configuration to rectangular. . . . . . . . 59

4.4 Influence influence of image vortex . . . . . . . . . . . 62

4.5 Vortex passing through compressibility induced $\nabla G_{c} \ldots \ldots$

4.6 Sketch acoustic lumped element model . . . . . . . . . . . . 64

4.7 Lumped element model, symmetric $\Delta B_{c}^{\prime}$ and $\Delta B_{r}^{\prime}$ for $L_{\text {eff }}=0.66$

4.8 Lumped element model, symmetric $\Delta B_{c}^{\prime}$ and $\Delta B_{r}^{\prime}$ for $L_{\text {eff }}=1.67$

4.9 Example of right angle inlet nozzle mesh . . . . . . . . . . . . 69

4.10 Initial condition base flow for $M=0.197 \ldots \ldots \ldots$. . . . 71

4.11 Snapshots of vortex during the generation process . . . . . . . 73

4.12 Definition of relative coordinates for momentum source f . . . . 74

4.13 Generation phase of vortex circulation $\tilde{\Gamma}=-0.86 \ldots \ldots 75$

4.14 The vorticity $\omega_{z}$ distribution at different stages of vortex generation for a vortex of $\tilde{\Gamma}=-0.86 \ldots \ldots \ldots \ldots$

4.15 Vortex passing through a right angle inlet nozzle . . . . . . . . 77

4.16 Location of the probe used to record the upstream acoustic response due to vortex-nozzle interaction . . . . . . . . . . 78

4.17 Integrated nozzle influence of vortex-core radius $R_{\Gamma} \ldots \ldots$. . 80

4.18 Integrated nozzle influence of vortex release height $h \ldots \ldots 1$

4.19 Integrated nozzle influence of dimensionless circulation $\tilde{\Gamma} \ldots . .82$

4.20 Integrated nozzle influence of dimensionless circulation $\tilde{\Gamma}= \pm 0.10883$

4.21 Integrated nozzle inlet influence of Mach number $M \ldots$. . . 85

4.22 Integrated nozzle inlet influence of Mach number $M \ldots$. . . . 86

4.23 Sketch of transversal mode frequencies in spectrum . . . . . . . 86

4.24 Right angle nozzle inlet influence of vortex-core radius $R_{\Gamma} \ldots 87$

4.25 Right angle nozzle inlet influence of vortex release height $h$. . 89

4.26 Right angle nozzle inlet geometry influence of dimensionless circulation $\tilde{\Gamma} \ldots \ldots \ldots \ldots \ldots$

4.27 Right angle nozzle inlet influence of Mach number $M$. . . . . . 91

4.28 Right angle nozzle inlet influence of Mach number $M$. . . . . . 92

4.29 Stages of compression on nozzle wall . . . . . . . . . . . 94

4.30 Estimation of $L_{0}$ sketch . . . . . . . . . . . . . . 95

4.31 Residual length $L_{0}$ influence $h \ldots \ldots \ldots \ldots$

4.32 Influence of scaling with $\tilde{L}_{\text {eff }} \ldots \ldots \ldots \ldots \ldots$

4.33 Sketch $\sin ^{2}(\omega t)$ on $0 \leq \omega t \leq T \ldots \ldots \ldots \ldots \ldots$. . . . . 97

4.34 Sketch $\sin ^{2}(\omega t)$ on $0 \leq \omega t \leq T / 2 \ldots \ldots \ldots$. . . . . . 98

4.35 Lumped element model data fit $\tilde{\Gamma} \simeq-0.1 \ldots \ldots . \ldots . \ldots 100$

4.36 Lumped element model data fit $\tilde{\Gamma} \simeq-0.9 \ldots \ldots$. . . . . . . 101

4.37 Lumped element model data fit $\tilde{\Gamma} \simeq-0.1$ Mach varried . . . . . 102

4.38 Ramp nozzle inlet influence of vortex-core radius $R_{\Gamma} \ldots \ldots$. . . 103

4.39 Ramp nozzle inlet influence of vortex release height $h \ldots . . . .104$ 
4.40 Ramp nozzle inlet geometry transition to from linear $\tilde{\Gamma}$ to quadratic $\tilde{\Gamma}^{2}$ scaling . . . . . . . . . . . . . . 105

5.1 Time dependence of the frequency of pulsations observed in a SRM . . . . . . . . . . . . . . . . . 112

5.2 Axial injection cold gas scale experimental setup . . . . . . . . 114

5.3 Oscillation frequency with respect to the acoustic mode frequency (left) and relative rms pressure oscillation level (right) as a function of the Mach number . . . . . . . . . . . . . . 115

5.4 Schwarz-Christoffel transformation of backward facing step with uniform approach flow . . . . . . . . . . . . . . 121

5.5 Influence of cavity volume $V_{c}$ for $n=2 \ldots \ldots \ldots \ldots$

5.6 Influence of nozzle on the rms pressure as a function of the Mach

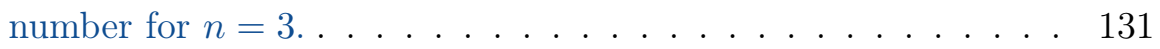

5.7 Influence of geometric parameters of the nozzle on model predictions. . . . . . . . . . . . . . . . . 134

5.8 Influence of the cavity volume $V_{c} \ldots \ldots \ldots \ldots$

6.1 Nozzle inlet geometries considered . . . . . . . . . . . . . . 140

6.2 Influence of upstream Mach number on upstream acoustic response: entropy-spot-nozzle interaction with and without nozzle cavity . . . . . . . . . . . . . . .

6.3 Influence of the entropy-spot-core radius on upstream acoustic response: entropy-spot-nozzle interaction with and without nozzle cavity . . . . . . . . . . . . . . . .

6.4 Influence of the upstream release height on entropy-spot-nozzle interaction acoustic response: with and without nozzle cavity . 145

6.5 Upstream acoustic response, for both a right angle nozzle inlet geometry and an integrated nozzle. . . . . . . . . . . . 147

6.6 P9 scale experiments with combustion, with and without a nozzle cavity after (Gallier et al. 2009). . . . . . . . . . . . . . 147

D.1 Abrupt contraction mapping . . . . . . . . . . . . . . . 171

E.1 Crighton analytical solution validation . . . . . . . . 177

G.1 Vortex passing through nozzle with cavity . . . . . . . . . . 182

I.1 Arbitrary control volume, drawing taken from (Hulshoff 2016b). 188

I.2 Example of numerical domain which is a truncated region of the physical domain with Inflow/Outflow and Wall boundary conditions. . . . . . . . . . . . . . . . . . . . 189

I.3 Subsonic inflow and outflow cases. . . . . . . . . . . . . . 191

I.4 Supersonic inflow and outflow cases. . . . . . . . . . . . . . 192 
I.5 Example of a symmetrical problem in which symmetry boundary conditions are used to reduce the size of the numerical domain. Figure taken from (Hulshoff 2016b). . . . . . . . . . . . 192

I.6 Overview of the numerical discretization technique. Figure taken from (Hulshoff 2016b). . . . . . . . . . . . . . . . . 193

I.7 Structured grid block (left) and mapping to a Cartesian domain (right). Figure taken from (Hulshoff 2016b). . . . . . . . . . . 193

I.8 Examples of neighboring blocks allowed in EIA . . . . . . . . . 194

I.9 Cell centred finite volume discretization. Figure taken from (Hulshoff 2016b). . . . . . . . . . . . . . . . . 195

I.10 Halo cells used for the enforcement of boundary conditions. Figure taken from (Hulshoff 2016b). . . . . . . . . . . . . 196

J.1 Example of right angle inlet nozzle mesh . . . . . . . . . . . . 199

J.2 Snapshots of entropy spot during and after the generation process 200

J.3 The normalized density distribution at different stages of entropy spot generation for an entropy spot of maximum deviation

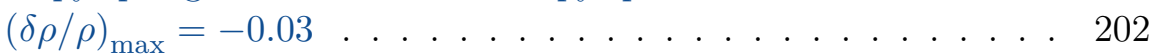

J.4 Relative density profile of entropy spot for different core radii. . 203

J.5 Shape of entropy spot for different upstream Mach numbers. . . 203 


\section{Nomenclature}

The following file gives the nomenclature :

\section{Latin Characters :}

$\begin{array}{llll}A & \text { Area } & m & \text { Mass flux } \\ B & \text { Total enthalpy } & \mathbf{n} & \text { Outer unit normal } \\ b_{n} & \text { Amplitude of nth mode } \psi_{n} & R & \text { Used for radius and re- } \\ c & \text { Sound speed } & & \text { flection coefficients speci- } \\ e & \text { Specific internal energy } & & \text { fied in the text } \\ \mathcal{E}_{t} & \text { Total energy density } & p & \text { Pressure } \\ f & \text { Frequency } & r_{p} & \text { Pipe radius } \\ \mathbf{f} & \text { Specific force density } & r_{o} & \text { Orifice radius } \\ G & \text { Green's function } & s & \text { Specific entropy } \\ F(z) & \text { Complex potential } & S_{1} & \text { Upstream channel height } \\ \mathcal{H} & \text { Heaviside function } & S_{2} & \text { Contraction throath height } \\ h & \text { Upstream vortex release } & T & \text { Oscillation period } \\ & \text { height also used for en- } & V_{c} & \text { Nozzle cavity volume } \\ & \text { thalpy } & V_{\text {eff }} & \text { Effective volume } \\ \text { i } & \text { Imaginary unit } & V_{0} & \text { Residual volume } \\ k & \text { Wavenumber } & \mathbf{v} & \text { Velocity vector } \\ L_{\text {eff }} & \text { Effective length } & v & \text { y-velocity component } \\ \mathfrak{L}_{\text {eff }} & \text { Effective chamber length } & \mathcal{Q} & \text { Volume line source } \\ L_{o} & \text { Distance between nozzle } & \mathcal{Q}_{E} & \text { Energy source term } \\ & \text { inlet and orifice } & U & \text { Upstream main flow veloc- } \\ L_{p} & \text { Pipe length } & & \text { ity } \\ \delta L_{p} & \text { Pipe length correction } & u & \text { x-velocity component } \\ M & \text { Mach number } & \mathbf{x} & \text { Observer coordinate } \\ m & \text { Hydrodynamic mode num- } & \mathbf{y} & \text { Source coordinate } \\ & \text { ber } & z & \text { Complex number }\end{array}$




\title{
Notation :
}

$(\ldots)^{\prime} \quad$ Fluctuation

(...) Dimensionless quantity

$(\ldots)^{*}$ Complex conjugate

$\Delta(\ldots)$ Difference

$\delta(\ldots)$ Difference

$(\ldots)_{0}$ Observer state value with the exception of $V_{0}$ which is the residual volume

$\langle\ldots\rangle \quad$ Time averaging over one period of oscillation

\section{Greek Characters :}

\begin{tabular}{|c|c|c|c|}
\hline$\delta_{n m}$ & Kronecker delta & $\tau$ & Time at which punctual \\
\hline$\delta(x)$ & Dirac delta function & & source is activated or \\
\hline$\epsilon$ & $\begin{array}{l}\text { Small value or numerical } \\
\text { error }\end{array}$ & & $\begin{array}{l}\text { source time coordinate, } \\
\text { unless otherwise specified }\end{array}$ \\
\hline$\Gamma$ & Vortex circulation & & in the text \\
\hline$\Phi$ & Scalar potential & $\rho$ & Density \\
\hline$\phi$ & Potential of incompress- & $\omega$ & Angular frequency \\
\hline & ible frictionless flow used & $\psi_{n}$ & nth mode \\
\hline & for Howe's compact low- & $\Psi$ & Stream function \\
\hline & frequency Green's function & $\omega$ & Vorticity \\
\hline & & $\zeta$ & $\begin{array}{l}\text { Complex number in zeta } \\
\text { plane }\end{array}$ \\
\hline
\end{tabular}

\author{
Abbreviations : \\ SRM Solid Rocket Motor \\ AVS Angle Vortex Shedding \\ DLR Deutsches Zentrum für Luft- und Raumfahrt e.V. \\ OVS Obstacle Vortex Shedding \\ SVS Surface Vortex Shedding \\ TVD Total Variation Diminishing
}




\section{Chapter 1}

\section{Introduction}

\subsection{Large solid rocket motors}

In this work the aeroacoustics of large solid rocket motors (SRMs) is considered. SRMs form part of launch vehicles such as Ariane 5 (Fig. 1.1), and are used as boosters for take off. Two boosters are used. Both have a propellant mass of approximately 230 tons, hence the name P230 where the P stands for "Poudre". These provide more than $90 \%$ of the thrust (combined $10^{4} \mathrm{kN}$ ) during take off (Anthoine 2000).

In a SRM, the oxidizer and fuel are mixed and cast as a propellant segment. The segments (S1, S2 and S3 in Fig. 1.2) occupy most of the volume of the combustion chamber. The propellant of Ariane 5 is composed of a hydroxyterminated polybutadiene, binder, aluminum powder, ammonium perchlorate as oxidizer and additives (Anthoine 2000). Fig. 1.2, shows the internal configuration of the P230 booster. It contains solid propellant segments separated by thermal inhibitors. Combustion occurs after ignition in a thin flame on the surface of the propellant. The first segment (S1) has a star shaped inner surface. It provides a high thrust at lift off. A picture of a $1 / 35$ scale version of this segment is shown in Fig. 1.3. Because of its large surface area, after ignition it is completely burnt within 40 seconds. Segments S2 and S3 are hollow cyclinders (instead of the star shape of S1) and burn for the remainder of the flight of the launcher with attached P230 boosters, continuing to provide thrust. Total flight time with boosters attached is about two minutes. During flight the inner surface of the propellant segments burns. This causes gas injection into the combustion chamber and resulting in a flow which exits through the downstream nozzle, which is choked. I.e. the Mach number of the flow at the nozzle throat is unity.

An integrated nozzle with nozzle cavity around the nozzle inlet is used. In Fig. 1.4, sketches of the nozzle at different stages of flight are shown. Just after 


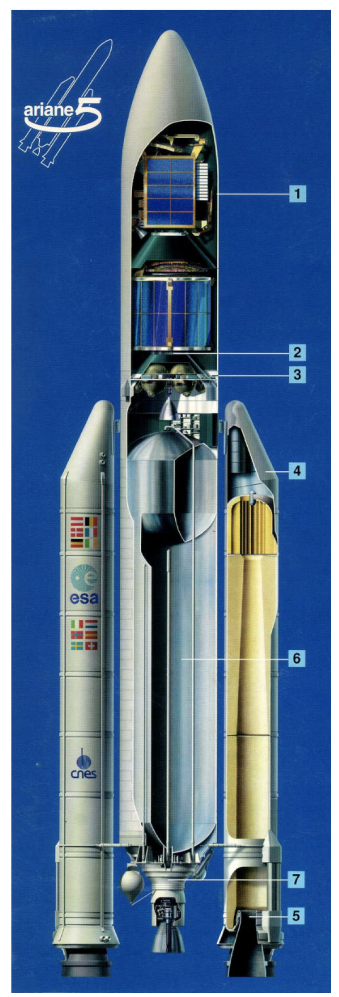

(a) Ariane 5

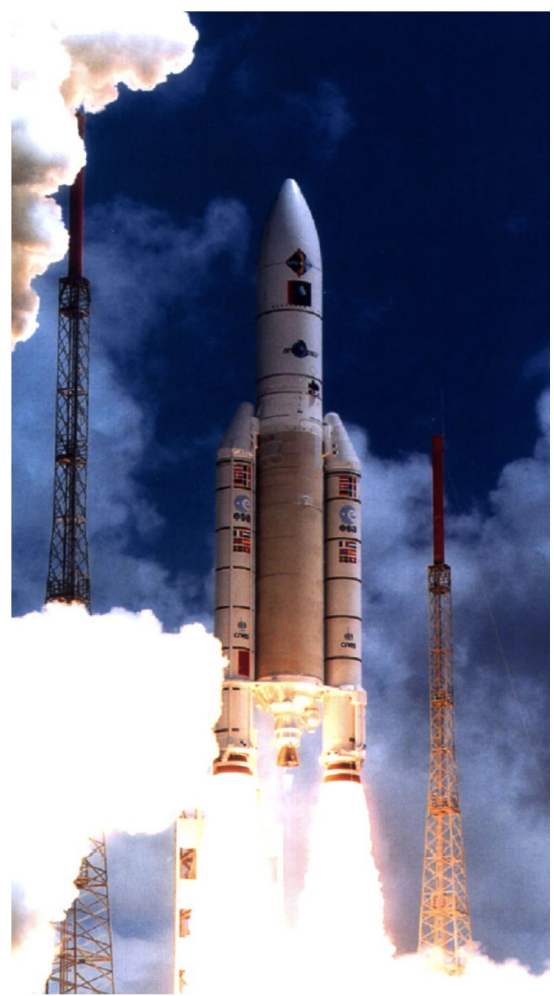

(b) Ariane 5 take off

Figure 1.1: Subfigure (a) 1: Fairing, 2: L9/EPS storable propellant stage, 3: Vehicle equipment bay, 4: P230 SRM booster, 5: Booster nozzle, 6: H155/EPC main cryogenic stage, 7: Vulcain engine. Subfigure (b) ignited after the Vulcain engine, the two P230 boosters deliver more than 90\% of total thrust at take off. Pictures taken from (Anthoine 2000).

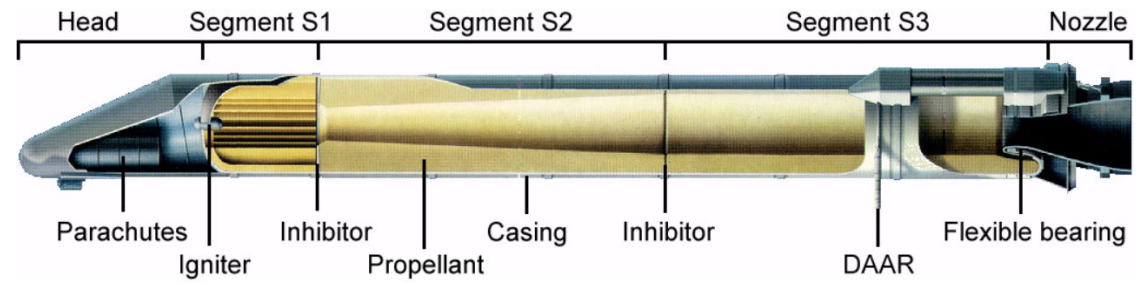

Figure 1.2: Ariane 5 P230 SRM. Picture taken from (Anthoine 2000) 


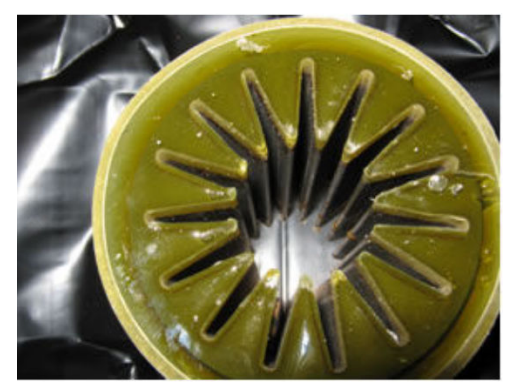

Figure 1.3: Star-shaped S1 propellant segment of a P230 1/35 scale motor. Picture taken from (Doisneau 2013)

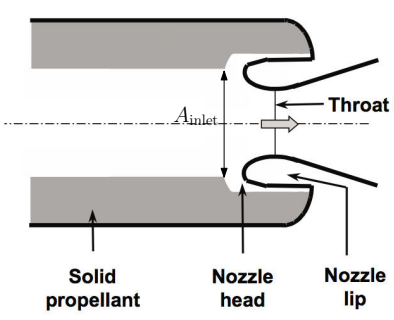

(a) Beginning launch

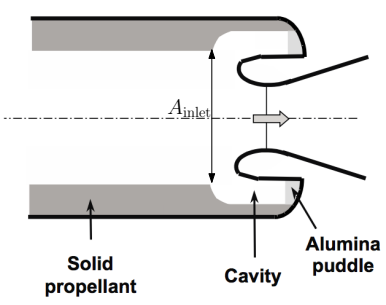

(b) During flight

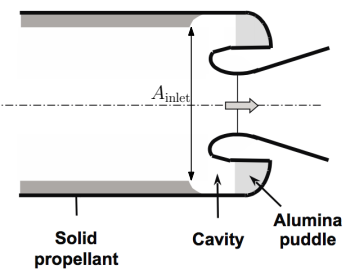

(c) Near the end of flight

Figure 1.4: Propellant regression during different stages of flight. The variation of the acoustically available cavity volume during flight is shown. Pictures taken from (Anthoine 2000). 


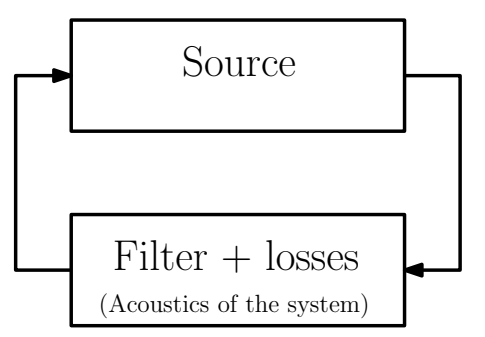

Figure 1.5: Feedback loop in self-excited systems.

ignition, the nozzle cavity is filled with solid propellant (Fig. 1.4 (a)). Using the fact the throat is choked the Mach number at the nozzle inlet can be estimated. It is in quasi-steady approximation determined by the geometry of the nozzle (Thompson 1972). The solid propellant surface regresses as it burns changing the nozzle inlet geometry. Namely, the cross-sectional surface of the inlet $A_{\text {inlet }}$ increases. This causes the inlet Mach number $M \equiv U / c$ ( $U$ average axial inlet flow velocity and $c$ the local speed of sound) to decrease because the crosssectional area of the throat remains almost constant (Anthoine 2000). Thus, due to the combustion of the propellant the inlet Mach number $M$ decreases as a function of flight time. After about a minute, a large part of the propellant has burned around the nozzle inlet and the cavity is filled with gas (Fig. 1.4 (b)). Near the end of the flight, aluminium-oxide droplets have accumulated in the cavity reducing the volume accessible to gas (Fig. 1.4 (c)).

\subsection{Statement of the problem}

After approximately one minute of flight, spurious sustained low frequency pressure pulsations are observed in the P230 boosters of Ariane 5. These pulsations are due to standing longitudinal acoustic waves in the combustion chamber of the SRMs. While the acoustic pressure fluctuations are only on the order of $10^{-3}$ of the static pressure in the chamber, the force associated with these pressure pulsations is approximately 5\% of the thrust (Fabignon et al. 2003; Anthoine 2014). To avoid that pulsations damage the payload, mechanical damping devices are installed, levying a cost on the amount of payload transported per flight. It is thus imperative that the nature of these pressure pulsations be understood. Indeed, a good grasp of what fundamentally drives these pulsations might allow one to take remedial action.

It should be noted that only a small part of the available energy produced by combustion in a SRM is involved in the establishment of sustained pressure pulsations. These sustained pressure pulsations are self-sustained. Namely, a feedback loop exists in which a source of sound is coupled to the acoustics of the system, see Fig. 1.5. The source can be due to direct or indirect combustion noise. Direct combustion noise is related to the chemically reactive region viz. 
where chemical kinetics takes place. Direct combustion noise is due to fluctuations in combustion rate mainly driven by pressure or velocity fluctuations (Culick 2006; Gallier and Godfroy 2009; Genot et al. 2017; Genot et al. 2018). Indirect combustion noise concerns the interaction of flow field inhomogeneities such as vortices and entropy spots with the nozzle as a source of sound (Bake et al. 2009; Kings and Bake 2010; Kings 2014; Dowling and Mahmoudi 2015; Morgans and Duran 2016). In SRMs direct combustion sound sources are:

- unsteady surface combustion of the propellant grain (Kuentzmann et al. 2004; Culick 2006),

- unsteady volume combustion of Aluminum particles (Dupays 2002; Culick 2006; Gallier and Godfroy 2009; Kuentzmann et al. 2004; Genot et al. 2017; Genot et al. 2018),

and indirect combustion noise sources are:

- convection of an entropy inhomogeneity through the nozzle (Bake et al. 2009; Morgans and Duran 2016),

- vortex $^{1}$ shedding leading to vortex-nozzle interaction (Vuillot 1995; Dotson et al. 1997; Anthoine 2000; Culick 2006).

Direct combustion noise as a source has been the main focus of study as established in the literature (Culick 2006; Kuentzmann et al. 2004; Gallier and Godfroy 2009; Genot et al. 2017; Genot et al. 2018). However, research by ONERA (Hijlkema et al. 2011), ArianeGroup (Gallier et al. 2009) and the von Karman institute (VKI) have demonstrated using scale model experiments (Anthoine et al. 2015), numerical simulations (Vuillot 1995; Vuillot and Casalis 2004; Anthoine et al. 2003; Gallier et al. 2009) and cold-gas-scale experiments (Anthoine 2000), that vortex shedding followed by vortex-nozzle interaction can induce pulsation amplitudes of the order of pulsations observed in full scale SRMs. Cold-gas-scale experiments will be discussed later in more detail. As will be explained later, the time evolution of the oscillation frequency provides a clue that limit-cycle pulsations are driven by a feedback loop involving the convection of perturbations (entropy spots or vortices) before these produce sound by interaction with the nozzle.

The "exact" description of the flow and combustion in a large SRM is an extremely difficult task. Even if the equations describing the flow can be formulated they involve a enormous number of empirical parameters which are difficult to determine accurately. In general complex processes such as combustion are described by simplified models (Vervisch et al. 2017). Even if all the processes and parameters are accurately known the flow remains very difficult to predict because of the nonlinearity of the equations. This nonlinearity im-

\footnotetext{
${ }^{1} \mathrm{~A}$ vortex is a coherent spatial vorticity distribution $\omega$. Vorticity is by definition the rotation of a velocity field $\mathbf{v}$ viz. $\boldsymbol{\omega} \equiv \nabla \times \mathbf{v}$. It is twice the angular velocity of the local spin of a fluid particle. The strength of a vortex is characterized by its circulation $\Gamma$. Where $\Gamma=\int_{C} \mathbf{v} \cdot \mathrm{d} \mathbf{x}=\int_{S} \boldsymbol{\omega} \cdot \mathbf{n} d^{2} x$, where surface $S$ is enclosed by the contour $C$ (Prandtl and Tiejens 1934; Saffman 1992).
} 
plies chaotic solutions such as turbulence (Landau and Lifshitz 1987; Kundu 1990; Piomelli et al. 2018). According to Fabigion et al. (Fabignon et al. 2016) an "exact" description of turbulence in a full scale large SRM, using a highly simplified model in which e.g. combustion of the propellant is replaced by injection of a non-reactive gas (cold gas) through walls, would involve calculations with $10^{11}$ grid points to resolve the smallest length scales of turbulence. That being said, calculations with $10^{9}$ grid points appear to provide reasonably satisfactory results (Fabignon et al. 2016).

Indeed, the global flow behavior is not very sensitive to the finest details of the flow when one is mainly interested in global flow oscillations and associated pressure oscillations. This is because the phenomenon considered is not critically sensitive to the Reynolds number $R e$ of the flow as long as it is sufficiently large. In the case of a SRM the Reynolds number can be determined using $R e=U D / \nu$ here $U=M c$ is the average velocity upstream from the nozzle inlet, the diameter $D$ of the combustion chamber and $\nu$ the kinematic viscosity.

To illustrate insensitivity of flow behavior with respect to Reynolds number consider the flow around a long cylinder placed normal to a steady uniform flow (Achenbach and Heinecke 1981). There are different critical values of Reynolds number $R e_{\text {cylinder }}=U_{\infty} D_{\text {cylinder }} / \nu$ (average upstream velocity $U_{\infty}$, cylinder diameter $D_{\text {cylinder }}$ and kinematic viscosity of the fluid $\nu$ ) distinguishing drastically different flow behavior. Around $R e=10^{2}$ the wake becomes unstable and a so-called von Kármán vortex street appears consisting of vortices shed with alternating circulation. In the region of $R e=10^{3}$ the wake becomes turbulent with a laminar viscous boundary layer on the cylinder. Around $R e=10^{5}$ the viscous boundary layers at the surface of the cylinder become turbulent with a turbulent wake. In the range $10^{3} \leq R e \leq 10^{5}$ the flow is only weakly dependent on the Reynolds number, at least the dimensionless vortex shedding frequency $S t=f_{\text {shedding }} D_{\text {cylinder }} / U_{\infty}$ (Strouhal number) remains quasi-constant (Fig. 1.6).

When performing reduced-scale-model experiments as described by Hijlkema et al. (Hijlkema et al. 2011) one fundamentally assumes that global pulsation of the flow in a SRM is not critically sensitive to the Reynolds number. Costs and complexity are very significantly reduced when considering a reduced-scalemodel experiment instead of doing test using a full-scale SRM. However, it is important to realize that scale models will not always produce results that can be directly scaled up to a full-scale systems. An example of a failure is reported by Bruggeman et al. (Bruggeman et al. 1991). Nonetheless scale-model experiments are useful to advance basic understanding of the role of various parameters, for instance the influence of a cavity surrounding the nozzle inlet (Gallier et al. 2009; Hijlkema et al. 2011) passive remedial measures (Ribereau 


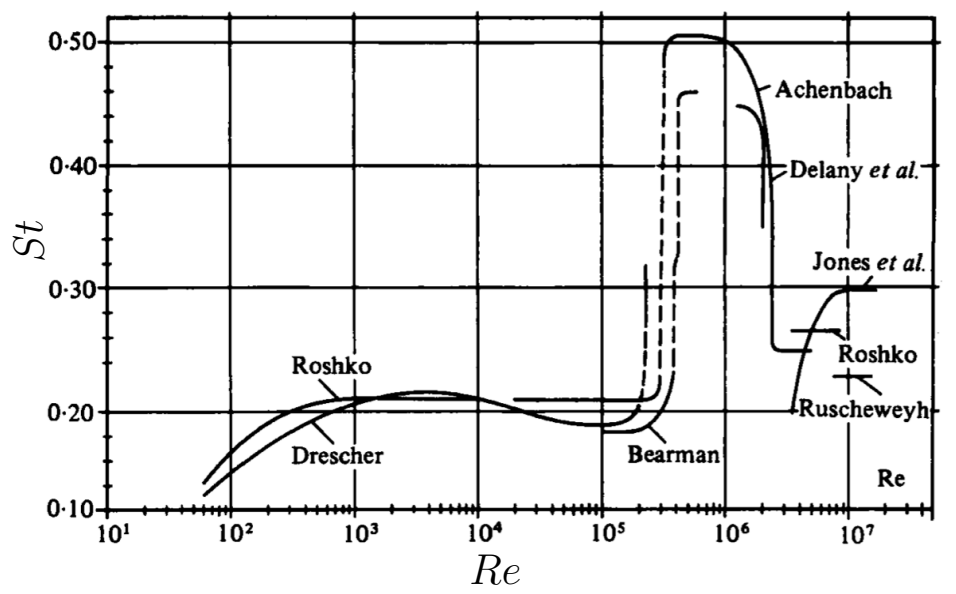

Figure 1.6: Strouhal number St as a function of Reynolds number Re for a smooth cylinder placed normal to a uniform flow. Figure taken from (Achenbach and Heinecke 1981)

et al. 2005) or variable fuel grains burn velocities (Anthoine et al. 2015).

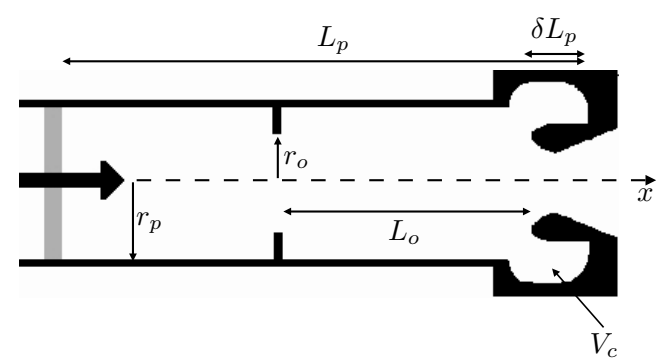

Figure 1.7: Scale model with axial injection through the upstream porous wall and an integrated nozzle at the downstream side. Figure taken form Anthoine (Anthoine 2000). In these experiments the volume $V_{c}$ around the nozzle inlet was varied. Anthoine also studied the effect of the chamber length $L_{p}$, orifice radius $r_{o}$, orifice position $L_{o}$ and Mach number $M$. The Mach number was varied by partial obstruction of the nozzle throat by means of a needle.

Further reduction in costs and complexity is achieved when cold-gas scale-model experiments are performed. In these experiments the flow field normally caused by burning propellant in SRMs is modeled by means of air injection through porous walls. Seminal cold flow experiments were carried out by Anthoine (Anthoine 2000) on a $1 / 30$ scale model for Ariane 5. Two types of experiments were carried out:

1. axial injection experiments (air is injected through a porous head end wall of the "combustion" chamber, a sketch is shown in Fig. 1.7),

2. radial injection experiments (air is injected through a porous side wall of 
the chamber).

The pressure difference across the porous walls used was so large that these can be assumed to behave acoustically as almost perfect reflecting walls. In axial injection experiments, the steady flow velocity is constant along the pipe axis. In radial injection experiments, the flow velocity increases linearly with the distance from the upstream pipe termination. Anthoine (Anthoine 2000) observed pressure pulsation amplitudes $p^{\prime}$ of the order of one thousandth of the static pressure $p$ in the "combustion" chamber. This is of the order of pulsations observed in actual large SRMs (Fabignon et al. 2003; Anthoine 2014). The key feature of these experiments is an integrated nozzle (Fig. 1.7) with variable cavity volume at the downstream end. This corresponds to the actual configuration of SRMs in Ariane 5. At ignition, the cavity is filled with solid propellant (Fig. 1.4 (b)). After about a minute, a large part of this propellant has burned around the nozzle inlet and the cavity is filled with gas (Fig. 1.4 (b)). Later, aluminium-oxide droplets captured by the cavity reduce the acoustically accessible cavity volume (Fig 1.4 (c)). Anthoine (Anthoine 2000) found that the relative pressure pulsations $p^{\prime} / p$ (pressure fluctuations over the static pressure in the chamber) where proportional to the volume of the cavity around the nozzle inlet. This could explain the delayed appearance of pulsations to one minute after combustion starts. The importance of the nozzle cavity volume has been confirmed in LP9 scale experiments with combustion of non-metallized solid propellant (Gallier et al. 2009; Hijlkema et al. 2011) and numerical simulations (Anthoine 2000; Gallier et al. 2009; Stella and Paglia 2011). Later Anthoine and Lemma (Anthoine and Lema 2009) used cold-gas-scale experiments to test passive control solutions. This approach has also been used to study fundamental aspects of surface vortex shedding (Kuentzmann et al. 2004; Gazanion et al. 2013).

A limitation of most scale-model experiments is that only measurements of the static pressure and (acoustic) pressure oscillations can be obtained at a few points in the combustion chamber. This is true for experiments involving combustion of propellant as well as cold-gas-scale experiments. Numerical simulations are a valuable tool to provide complementary information. When tuned to reproduce experiments one can assume that these provide information on details of the internal flow field.

As explained earlier, even without combustion an "exact" numerical solution of the equations describing the flow is impossible in view of the enormous numerical effort involved (Fabignon et al. 2016). In general trying to use the same numerical model and approximation for the entire flow is inefficient (Hulshoff 2016a). In large SRMs sound production occurs in the nozzle region which is small compared to the length of the combustion chamber. Within the nozzle region the nonlinear convective effects are essential but the acoustic wave propagation effects are not. Upstream from the nozzle in the combustion chamber 
the pressure perturbations propagate as acoustic waves, which have a relatively small amplitude $\left(p^{\prime} / p=\mathcal{O}\left(10^{-3}\right)\right)$. A linear theory for wave propagation can be used. The use of different theoretical approximations in different regions of a flow is standard practice in fluid mechanics (van Dyke 1964). The systematic use of this approach has been introduced by Prandtl (Prandtl 1904; Schlichting 1979), at the beginning of the $20^{\text {th }}$ century, to model separately and match the viscous flow near walls (boundary layers) with the effectively frictionless flow outside of the boundary layers. This is the spirit of the aeroacoustic hybrid methods, in which a certain approximation is used to describe a sound producing region and another is used to describe the radiated sound field far from the source region (Colonius and Lele 2004).

In the present work this approach is applied to the feedback loop in Fig. 1.5. Elementary processes in the feedback loop are isolated, then studied and modeled separately. For each process a specific theoretical or numerical approach is used. An example of such an approach is the T-Burner experiment used to study the acoustic response of solid propellant (Price and Sofferis 1958; Culick 2006). T-Burner experiments have led to the development of one-dimensional models as proposed by Hart and McCurle (Hart and McClure 1959) and Culick (Culick 2006).

\subsection{Focus of the study}

It is striking that considerable effort has been made to study the formation of vortices (Vuillot 1995; Casalis et al. 1998; Kuentzmann et al. 2004; Gazanion et al. 2013; Cerqueira and Sipp 2014; Lacassagne 2017), various losses of acoustic energy (Culick 2006) and direct combustion noise (Kuentzmann et al. 2004; Culick 2006; Gallier and Godfroy 2009; Kuentzmann et al. 2004; Genot et al. 2017; Genot et al. 2018). Here indirect sound production due to both vortex-nozzle and entropy-inhomogeneity-nozzle interaction is focused on. Entropy-inhomogeneity-nozzle interaction as a source of indirect sound has been investigated widely, albeit without a focus on SRMs as an application, both experimentally (Bake et al. 2009; Kings 2014; Tao et al. 2017) as well as theoretically (Marble and Candel 1977; Ffowcs Williams and Howe 1975; Leyko, Moreau, Nicoud, and Poinsot 2011). Apart from the pioneering work by Hulshoff et al. (Hulshoff et al. 2001), Kings et al. (Kings and Bake 2010; Kings 2014), no conclusive systematic study on vortex-nozzle interaction as a sound source has been found in the literature. Thus advancing the state of the art with regard to this mechanism was one of the aims of the research project reported here.

If the feedback loop (Fig. 1.5) is linearly unstable, after an initially exponential growth stage at low amplitudes, an essentially nonlinear process saturates 
growth causing the system to reach a limit cycle. Linear models predict the exponential growth but cannot $^{2}$ predict a limit cycle. When the limit cycle is stable, periodic pulsations result. This implies a balance between the time average over a period of acoustic power produced ${ }^{3}\left\langle\mathcal{P}_{\text {source }}\right\rangle$ and the acoustic power dissipated $\left\langle\mathcal{P}_{\text {loss }}\right\rangle$. In most cases a stable limit cycle involves a single dominant acoustic standing wave (mode of the combustion chamber). The oscillation frequency is close to the natural frequency of this mode (acoustical oscillation frequency without sources nor losses, due to initial perturbations).

In the case of vortex-nozzle interaction the saturation mechanism is the nonlinearity of the vortex shedding process, which occurs upstream from the nozzle. When vortex shedding occurs in the moderate amplitude regime (Bruggeman et al. 1991), the vortex circulation of the mature vortex which arrives at the nozzle will be independent of the amplitude of the pulsation. A linear theory of the flow perturbation would predict a "vortex" circulation proportional to the pulsation amplitude.

\subsection{Literature review}

Here phenomena reported in the literature are discussed. This discussion should provide some indication of the limits of the work presented in the rest of this work.

\subsubsection{Nonlinear effects and one-dimensional models}

At the limit-cycle, acoustic wavelengths encountered in SRMs are of the order of the length of the combustion chamber, which is much larger than its diameter. Thus, the traveling pressure waves are plane and can be described using a one-dimensional model.

However, such waves are not always accurately described by linear theory (acoustics). An example of a nonlinear effect is pressure wave steepening due to the increase of the speed of sound $c$ with the temperature $(c \propto \sqrt{T}$ with $T$ the absolute temperature) combined with the increase convection due to $u^{\prime}$ (wave speed is $U+u^{\prime}+c$ ). This effect is similar to the deformation of shallow water waves approaching the shore. After some distance the top of the water wave overtakes the foot, implying that at one position there are three water levels causing wave breaking (Thompson 1972). In a compression wave one cannot have three different values of pressure. Hence, extreme wave steeping will lead to the formation of a traveling pressure discontinuity called a shockwave. Due to the extremely large velocity and temperature gradients across a shockwave it

\footnotetext{
${ }^{2}$ This is by definition as the exponential growth keeps on indefinitely in a linear model.

${ }^{3}$ The brackets $\langle\cdots\rangle$ indicate time averaging over a period of oscillation.
} 


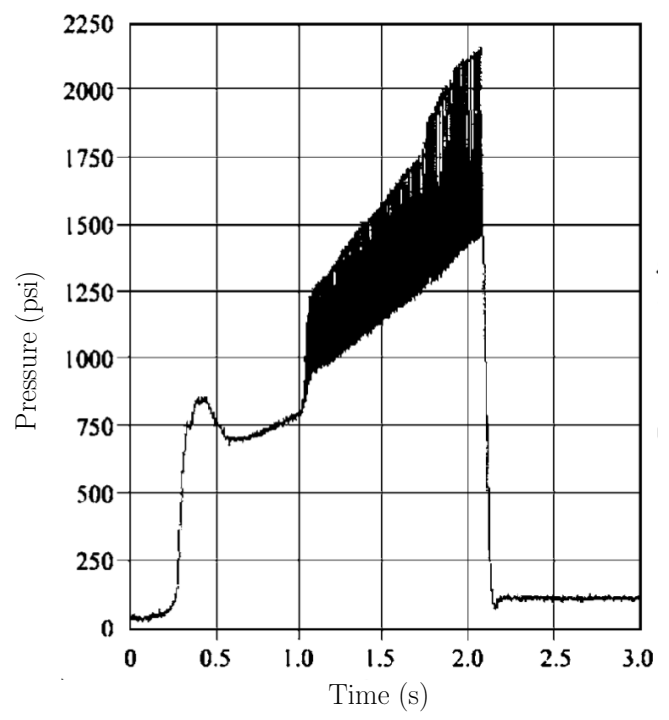

Figure 1.8: Small SRM firing reported by Flandro et al. (Flandro, Fischbach, and Majdalani 2007), displaying pulsation levels of $p^{\prime} / p=\mathcal{O}\left(10^{-1}\right)$. Note that, in contrast to data by Dotson et al. (Dotson et al. 1997) and Antoine (Anthoine 2000) no pulsation amplitude "bumps" are not observed, indicating that here vortex driven indirect sound does not play a role. Reproduction of Fig. 9 (a) in (Flandro, Fischbach, and Majdalani 2007).

involves dissipation of energy. The power dissipated scales with $\left(p^{\prime} / p\right)^{3}$ (Pierce 1989). If $p^{\prime} / p=\mathcal{O}\left(10^{-1}\right)$ as in small (tactical) SRMs e.g. Fig. 1.8, this can cause limit-cycle saturation and is the main mechanism considered in the literature (Levine and Baum 1982; Baum and Levine 1986; Culick 2006; Flandro et al. 2007; Greatrix 2011). Baum and Levine (Baum and Levine 1986) as well as Greatrix (Greatrix 2011) consider limit-cycle feedback loop caused by a pressure coupling of the flame on the surface of the propellant and the acoustic standing wave. These authors propose one-dimensional models which describe limit-cycle saturation due to shock formation in the case of direct combustion instability.

Ferretti et al. (Ferretti et al. 2011; Ferretti 2011) propose a one-dimensional nonlinear model of a SRM with a vortex sound source. Closer inspection of the publications by Ferretti et al. (Ferretti et al. 2011; Ferretti 2011) shows that production of sound is assumed to be due to the variation (gradient) of the cross section (at the nozzle of the SRM) but no discernible physical justification is provided for the approach. The presence of a nozzle cavity inlet viz. the absence of a single valued cross-sectional area, would imply the introduction of singularity in this modeling approach. As such it is unclear if this model is able to predict the important effect of the cavities around the nozzle observed by Antoine (Anthoine 2000). 
In a one-dimensional standing acoustic wave with pressure fluctuation of amplitude $p^{\prime}$ at pressure antinodes the particle velocity fluctuation $u^{\prime}$ at pressure nodes will have the magnitude $u^{\prime}=p^{\prime} / \rho c$ as $1<\gamma<1.4$ this implies that the ratio $u^{\prime} /(M c)$ of acoustic particle velocity and main flow velocity is, given by $p^{\prime} /(\gamma p M)$ with $\gamma \equiv \frac{C_{p}}{C_{v}}$ the ratio between heat capacity at constant pressure $C_{p}$ and volume $C_{v}$. This implies that for $p^{\prime} / p=\mathcal{O}\left(10^{-1}\right)$ one has $u^{\prime} /(M c)=\mathcal{O}(1)$. When considering vortex shedding nonlinear behavior is to be expected. This involves power losses scaling with at most $\left(u^{\prime} / c M\right)^{3}$ (Howe 1984) which can be a cause of saturation due to increased losses and thus attaining a limit-cycle. It is noteworthy that in the literature this important effect is not taken into account for small SRMs for which $p^{\prime} / p=\mathcal{O}\left(10^{-1}\right)$. Pressure pulsation amplitudes in large SRMs are relatively small $p^{\prime} / p=\mathcal{O}\left(10^{-3}\right)$ for large SRMs (Anthoine 2000; Fabignon et al. 2003). Thus one can assume that acoustic wave propagation is linear.

The nozzle inlet Mach number is low $0.05 \leq M \leq 0.2$ (Anthoine 2000). In a standing wave, convective effects on wave propagation of the incoming wave is partially compensated by the opposite convective effects on the outgoing wave. Hence, convective effects on acoustic wave propagation can in first-order be neglected (Wang and Kassoy 1992). However, convective effects are important to take into account for acoustic radiation losses at the nozzle (Culick 2006).

In large SRMs limit cycles are reached due to the essentially nonlinear character of the sound source. The nonlinearity, believed to be responsible for the amplitude saturation, is that once as a result of flow instability the distributed vorticity has concentrated into a vortex (coherent structure) it cannot concentrate further and its circulation is independent of the acoustic oscillation amplitude (in moderate amplitude regime (Bruggeman et al. 1991)). Estimating the largest possible vortex circulation is a step in a method to estimate the largest pulsation amplitudes than can occur. This is the approach taken in this work.

\subsubsection{Vortex driven self-sustained pressure oscillations in SRMs}

There are three possible causes of vortex shedding in SRMs (see Fig. 1.9):

- Obstacle vortex shedding (OVS), which occurs as a result of flow separation at obstacles in the flow such as thermal inhibitors placed between segments of propellent grain (Anthoine 2000; Culick 2006; Kuentzmann et al. 2004).

- Angle vortex shedding (AVS), occurs in case of flow separation at an abrupt increase of combustion chamber cross section in the main flow direction (Vuillot 1995; Fabignon et al. 2016). 


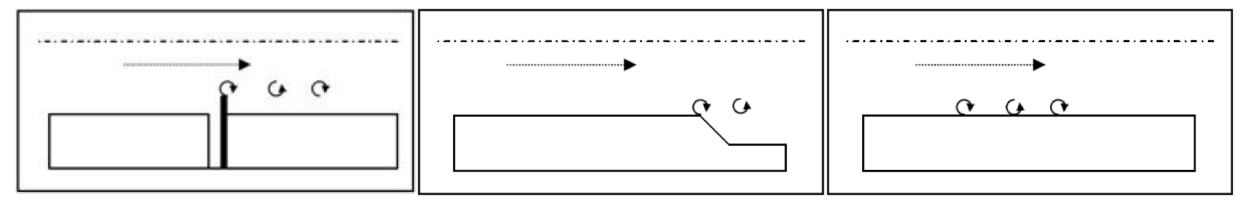

Figure 1.9: From left to right: obstacle vortex shedding (OVS), angle vortex shedding (AVS) and surface vortex shedding (SVS).

- Surface vortex shedding (SVS), due to the intrinsic instability of the Taylor-Culick flow generated by surface combustion of the propellant resulting in the concentration of existing vorticity (Casalis and Vuillot 2004; Fabignon et al. 2016; Vuillot and Casalis 2004).

Regardless of the mechanism by which the vortices are created, the formation of vortices triggered by the acoustic oscillation implies sound absorption. The created vortices are then convected towards the nozzle and it is their interaction with the nozzle that produces sound, which can drive self-sustained oscillations. The convection time from the point where vortex formation is initiated, to the nozzle, is referred to as the convective delay time. For critical ratios of delay time to acoustic oscillation period ${ }^{4}$, the sound generated by the source reinforces the oscillations. As shown in Fig. 1.10 (results for Titan 4 SRM (Dotson et al. 1997)) the pulsation displays several amplitude maxima at frequency around the frequency of the first longitudinal acoustic mode (chamber length is half a wavelength). The frequency decreases around each amplitude maximum with decreasing Mach number at the inlet of the nozzle (this corresponds with increasing time). This can be explained by assuming that the flow is quasisteady. As the nozzle is critical, the upstream Mach number $M$ at the nozzle inlet is determined by the ratio of the combustion chamber cross-sectional area to nozzle throat cross-sectional area. The Mach number decreases with time, due to the increase of the combustion chamber diameter, with the regression of the propellant surface. The throat diameter of the nozzle remains approximately constant. Similar hydrodynamic modes appear in scale experiments with burning propellant carried out by ONERA (Gallier et al. 2009; Anthoine et al. 2015), in cold-gas scale experiments (Anthoine 2000) and in P230 Ariane 5 boosters see Fig. 1.11. The frequency signature as a function of time with succesive hydrodynamic modes has been recognized as a clue that sound generated by vortices is important in large SRMs (Dotson et al. 1997; Anthoine 2000; Fabignon et al. 2003; Anthoine 2014).

Each maximum of the pulsation amplitude with time corresponds to a hydro-

${ }^{4}$ This corresponds to so-called hydrodynamic modes $m_{h}$ of the system. 


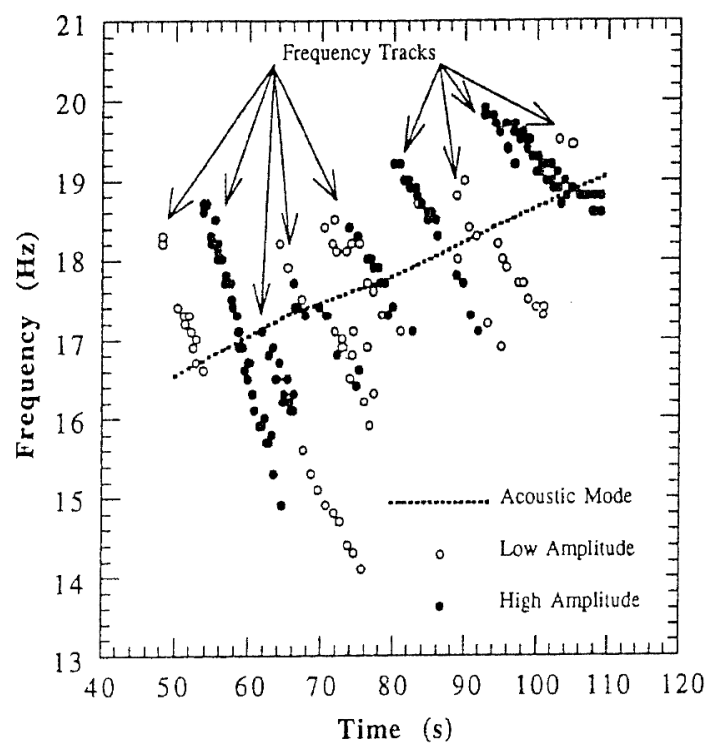

Figure 1.10: Titan 4 results, Fig. taken from Dotson et al. (Dotson et al. 1997). The Strouhal lines (frequency tracks) crossing acoustic mode line are evidence of the presence of vortices i.e. of the importance of hydrodynamic effects. Each frequency track line corresponds to a fixed number of vortices traveling downstream from the point of shedding to the inlet of the nozzle. The Mach number at the nozzle inlet decreases as a function of time due to the increase of combustion chamber diameter. These effects are common and have been observed and published recently e.g. see Fig. 13a in Anthoine et al. (Anthoine et al. 2015).

dynamic mode ${ }^{5}, m_{h}$. The ratio of Mach number ${ }^{6} M \equiv U / c_{0}$ and the difference $\Delta M$ between two successive modes provides an estimate of the number of vortices $m_{h} \simeq M / \Delta M$ present between the vortex shedding location and the nozzle inlet. Fig. 1.11 shows frequencies in a Ariane 5 P230 booster. According to Anthoine (Anthoine 2000) the lowest frequency track (hydrodynamic mode) line corresponds to $M=0.13$ and the top one to $M=0.07$. One can deduce that there are initially approximately five vortices $\left(m_{h}=0.07 / \frac{0.06}{4} \simeq 5\right)$ at onset of the pulsations. This is indeed confirmed by numerical simulations, in the case of OVS Vuillot et al. (Vuillot and Casalis 2004) find five vortices (see Fig. 1.12).

\footnotetext{
${ }^{5}$ The hydrodynamic mode $m_{h}$, corresponds to the vortex convection time plus the time of acoustical feedback divided by the natural acoustic oscillation period of the combustion chamber $m_{h}=\frac{f L_{\text {convection }}}{U}$. Since a new vortex is shed every period, $m_{h}$ corresponds to the number of vortices present between the vortex shedding point and the nozzle.

${ }^{6}$ Where $c_{0}$ is the speed of sound at the observer's position and $U$ a scale for the main flow velocity.
} 


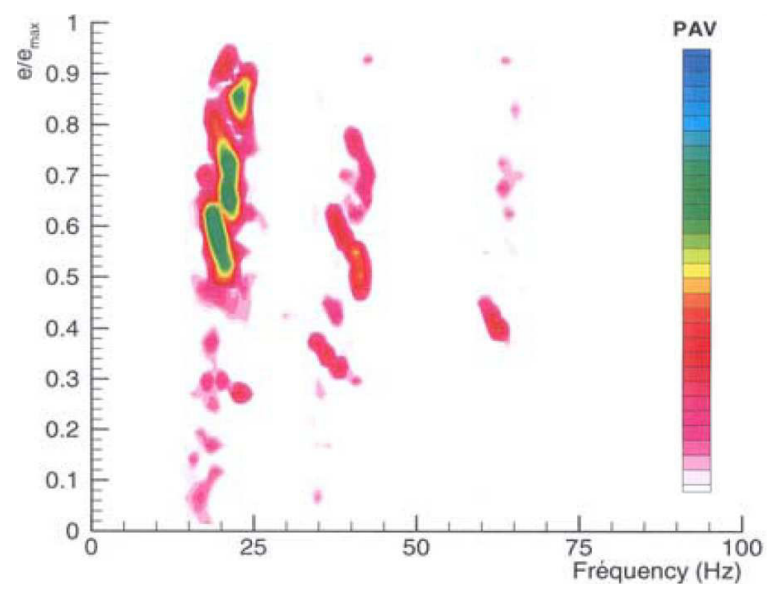

Figure 1.11: Figure taken from Fabignon et al. (Fabignon et al. 2003). Frequency tracks also called Strouhal lines in Ariane P230 boosters. According to Anthoine (Anthoine 2000) the lowest Strouhal (green lines around the first acoustic mode $20 \mathrm{~Hz}$ ) line corresponds to $M=0.13$ and the top one to $M=0.07$.

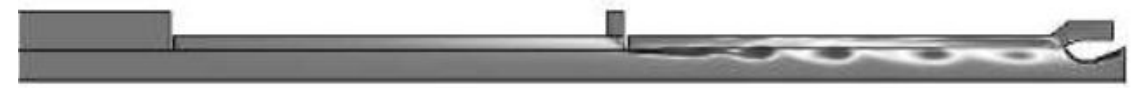

Figure 1.12: Figure taken from Vuillot et al. (Vuillot and Casalis 2004). Simulation of obstacle vortex shedding (OVS) in a LP3 motor, leading to five vortices traveling downstream from the inhibitor to the nozzle. This situation corresponds to $m_{h} \simeq 5$. 


\subsubsection{Aeroacoustic sound sources}

Aeroacoustics is the study of flow induced sound and more generally the interaction between flow and acoustic fields. In the following, first an illustrative example of sound production due to vortex dynamics is given. This is followed by a brief discussion of entropy-inhomogeneity driven sound production.

Consider the oscillating flow around a wing under incidence (with lift). A vortex with circulation $\Gamma$ is formed in the wake of the wing. When the incoming flow is started from rest and remains uniform, the total circulation in the viscous boundary layers around the wing will be equal and opposite to the circulation of the vortex in the wake. In a quasi-steady approximation, assuming a sufficiently low Strouhal number, the lift force on the wing (force normal to the incoming flow) has a magnitude proportional to the incoming flow velocity and the vortex circulation. It can be demonstrated that a force on the wing results in a reaction force of the wing on the fluid, which if it is unsteady, according to Curle is a source of sound (Curle 1953; Powell 1961). Hence, the vortex in the wake is not the source of sound. However from the time dependence of the circulation in the wake one can deduce the behavior of the hydrodynamic force on the wing. The reaction force of the wing on the fluid is the source of sound. When the wing is small compared to the typical acoustic wavelength of the radiated sound, the flow around the wing and in the nearby wake can be described by means of an incompressible approximation. Under free-field conditions the sound generated by the unsteady force from the wing on the fluid is a dipolar sound field. It does not correspond to a net change of volume of the fluid. In a duct at low frequencies, plane wave propagation prevails and such a dipole can be represented as a time-dependent pressure discontinuity in the one-dimensional acoustic field. This is an example of what is referred to as a lumped element aeroacoustic sound source model. This compact sound source approximation will be used chapter 3 when considering vortex nozzle interaction. Vortex sound interaction also occurs when a vortex is generated. In this case linear quasi-steady loss models will be used (chapter 5).

Entropy inhomogeneities convected through a contraction in a ducted flow are a source of sound. This was pointed out theoretically by Ffowcs Williams and Howe and coined acoustic "Bremsstrahlung" (Ffowcs Williams and Howe 1975; Howe 2014). If an entropy inhomogeneity (or entropy spot) has a small difference of density with respect to its surrounding fluid. It is convected with the flow. When the spot meets a contraction in the duct, a buoyancy force acts on it, pushing the spot through the contraction. This force induces a timedependent force on the walls of the contraction, which by virtue of Newtons third law induces a force on the fluid generating sound. The existence of this dipolar sound source was confirmed experimentally by Bake et al. (Bake et al. 2009; Bake et al. 2009). 


\subsubsection{Influence of propellant}

The properties of the propellant used in SRMs appears to play a significant role. In particular the effect of aluminum particles mixed in the propellant (Gallier and Godfroy 2009; Anthoine 2014; Genot et al. 2017; Genot et al. 2018). It appears that not only the amount of aluminum is important, but also the size distribution of the particles. It appeared from scale-model experiments that particles of density $\rho_{p}$ and diameter $D_{p}$ with a Stokes number $S t=\frac{\rho_{p} \pi f D_{p}^{2}}{9 \rho \nu}$ of order unity tend to increase the pulsation levels (Anthoine 2014). Aluminum particles contribute to the combustion and it is not surprising that they affect the stability of the process. An interesting result from numerical simulations is that passive particles also enhance pulsations for $S t=\mathcal{O}(1)$ (Guery et al. 2008; Anthoine 2014). This was verified using scale-model experiments (Hijlkema et al. 2011). These types of effects are not considered in this work.

\subsubsection{Hydrodynamic stability of a Taylor-Culick flow}

Due to the fact that pulsations without the presence of thermal inhibitors and abrupt angles can occur (Dotson et al. 1997), a considerable research effort was undertaken to study the stability of a Taylor-Culick flow in a pipe.

A Taylor-Culick flow is created in a cylinder of radius $R$ by a uniform gas injection through the side wall (Fig. 1.13). Due to the injection at the wall the flow velocity along the center line accelerates in the downstream $(x)$ direction while the fluid injected at the wall has for $x>>R$ a relatively low velocity. This results for $x>R$, even in the case viscosity is neglected, in a non-uniformaxial velocity profile $\left(u_{\text {axial }}=\pi x \cos \left(\frac{\pi}{2} r^{2}\right)\right.$ where $x$ and $r$ are the axial position and radius in polar coordinates, respectively). This non uniform flow profile implies the presence of vorticity in this flow. As this flow is quasi-parallel, the pressure in a cross section is uniform. There will therefore be an associated entropy difference between the fluids at the centreline and at the wall.

Considering a two-dimensional planar geometry Varapaev and Yagodkin (Varapaev and Yagodkin 1969) did pioneering work on the stability of such a flow indicating it becomes unstable for sufficiently high Reynolds numbers. The state of the art was considerably advanced in a series of papers by Casalis et al. (Casalis et al. 1998; Griffond and Casalis 2000; Griffond et al. 2000; Griffond and Casalis 2001; Casalis and Vuillot 2004; Chedevergne and Casalis 2006; Chedevergne et al. 2006; Chedevergne et al. 2012; Boyer et al. 2013; Boyer et al. 2013), who investigated various aspects of the instability of this flow. Cold-gas-scale models VECLA and VALDO, with a uniform duct crosssection and downstream open pipe termination, developed at ONERA allowed 

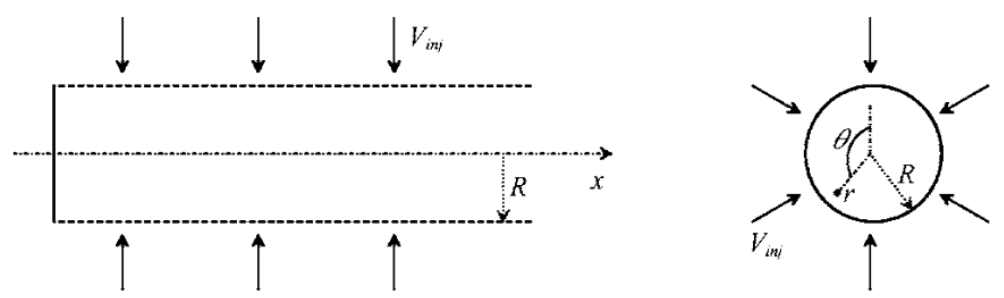

(a) Taylor-Culick flow generation.

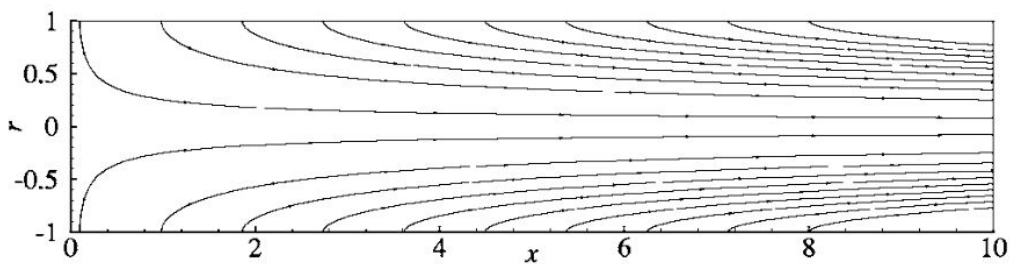

(b) Taylor-Culick flow

Figure 1.13: (a) Flow generated by injection of gas through the cylindrical wall located at $r=R$ with the injection velocity $V_{\mathrm{inj}}$. (b) The pipe is bounded by a solid wall located at $x=0$. Figures taken from (Chedevergne et al. 2006)

the experimental study of the instability of this flow. The theoretical and experimental contributions show that perturbations of the flow result in the propagation of vorticity waves (termed hydrodynamic modes in the literature not to be confused with the limit-cycle-hydrodynamic modes $m_{h}$ as defined in section 1.4.2) with growing amplitude as these travel downstream. After an initial perturbation, the perturbation of the flow decays in time as the vorticity waves are convected out of the pipe. Note also that, the finite length of the duct in the experiments presents a challenge for comparison between theory and experiments (Chedevergne and Casalis 2006; Chedevergne et al. 2006). In particular what boundary conditions to use at the downstream open pipe is not evident.

The linear perturbation analysis presented by Chevergne et al. (Chedevergne and Casalis 2006; Chedevergne et al. 2006; Anthoine 2014) has been compared to experimental results of SRM scale-model experiments LP9, LP10 and LP6 (Chedevergne and Casalis 2006; Chedevergne et al. 2006). This is done despite the fact that that there is no obvious application of stability theory for an open duct with radial injection results to systems displaying limit-cycle behavior. Chevergne and Casalis remark: "the link between the energy-balance method and the hydrodynamic stability is not fully understood yet, but matching the two approaches may result in a new way of characterizing the influence of the acoustic modes in solid rocket motors" (Chedevergne and Casalis 2006). 


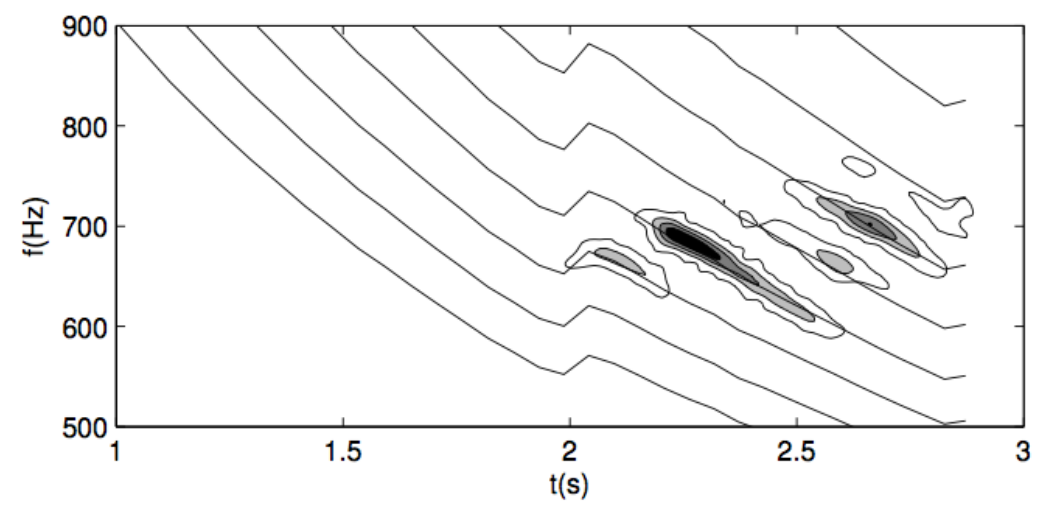

(a) Measured limit-cycle frequency $f$ compared to $\omega_{r} / 2 \pi$ as a function of time.

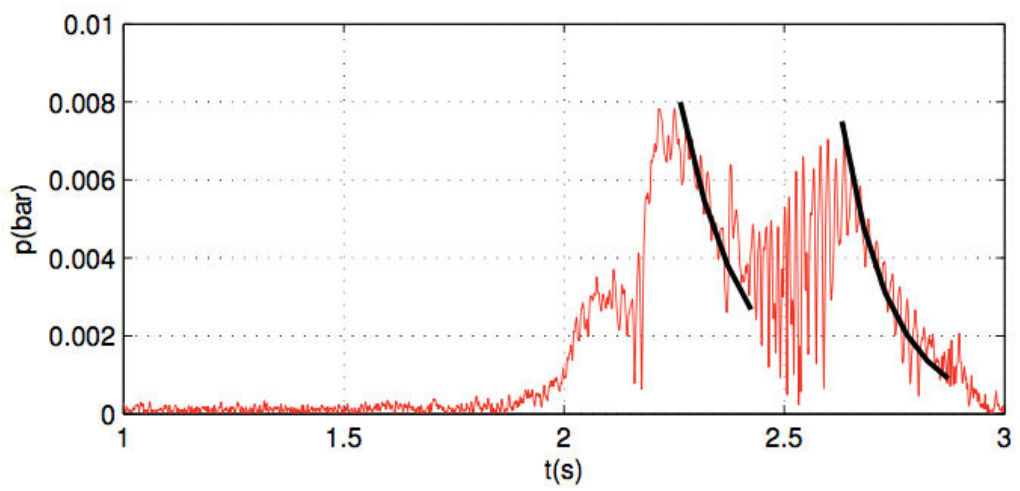

(b) Measured limit-cycle pressure signal $p$ compared to $\omega_{i}$ as a function of time.

Figure 1.14: Comparison of complex frequency $\omega=\omega_{r}+\mathrm{i} \omega_{i}$ derived from TaylorCulick flow stability analysis with LP9 scale-model data. Figures taken from (Chedevergne and Casalis 2006)

Chevergne and Casalis determine complex frequencies $\omega=\omega_{r}+\mathrm{i} \omega_{i}$ of instability modes (Chedevergne and Casalis 2006). Modes with $\omega_{i}<0$ decay exponentially in time as $\exp \left(\omega_{i} t\right)$. The local amplitude oscillates harmonically as $\exp \left(-\mathrm{i} \omega_{r} t\right)$. The complex frequency $\omega$ is obtained as a function of time for the experiments on the basis of an estimated geometry and flow conditions as a function of time. The real part $\omega_{r}$ is compared with the frequency of the limit-cycle pulsations observed in the scale-model experiments. The measured oscillation limit-cycle frequency $f$ is found to evolve in time as $\omega_{r} / 2 \pi$ for one of the hydrodynamic modes. In the experiments as well as in full-scale motors (Fig. 1.10 and 1.11) characteristic frequency signatures (frequency tracks) corresponding to limit-cycle-hydrodynamic modes $m_{h}$ are observed (Fig. 1.14 (a)). These hydrodynamic modes decay with time because the flow conditions 
and geometry evolve in time. The authors compare this decay in time with the calculated $\exp \left(\omega_{i} t\right)$ and find that it fits the time evolutions of some of the experimentally observed modes (Fig. 1.14 (b)). This seems to be coincidental.

Indeed, the evolution of the oscillation frequency with time in the experiment can be considered as a result of the time-dependence of the convective effect in the feedback loop (Fig. 1.5) describing the limit-cycle oscillation. As it happens, the magnitude of the variation in frequency with respect to the Mach number $\frac{M}{f} \frac{\mathrm{d} f}{\mathrm{~d} M}$ is determined by the acoustic losses of the system. This has clearly been demonstrated in similar self-sustained oscillations, described using a harmonic oscillator model for the amplitude (Tonon et al. 2011). Such a harmonic oscillator model will be derived for the present problem in chapter 2. Essentially when a limit cycle is reached the total phase shift of the perturbation traveling around the feedback loop (round trip time) should be an integer of $2 \pi$. Part of this phase shift is due to the convection of the vortices from the point where these are shed to the nozzle where sound is generated. When the frequency of oscillation matches the acoustic resonance frequency (of a dominant acoustic mode) the pulsation amplitude is maximized. When the Mach number increases the convective contribution to the round trip time decreases. The system compensates this by an increase in oscillation frequency. The increase in frequency implies that, the acoustic response of the resonator changes, contributing to the change of the phase delay in the feedback loop. Both effects contribute to maintain the sum of all phase shifts along the feedback loop equal to $2 \pi m$ (where $m$ is an integer characterizing the hydrodynamic mode of the oscillation). When the quality factor of the harmonic oscillator is large a minute increase in frequency induces a relatively large change in phase of the resonator response. Hence $\frac{f}{M} \frac{\mathrm{d} f}{\mathrm{~d} M}$ is very small. If the quality factor is low the change in frequency needed to compensate is larger. When the acoustic phase shift needed approaches $\pi / 2$ the system jumps to the lower hydrodynamic mode $2 \pi(m-1)$ corresponding to one vortex less in the flow. The system could also chose to jump to a higher acoustic mode, increasing the natural oscillation frequency.

When attempting to apply the approach of Chevergne and Casalis (Chedevergne and Casalis 2006) to a limit-cycle oscillation in a cold-gas-scale experiment at fixed Mach number $M$, it becomes clear that the $\exp \left(\omega_{i} t\right)$ timedependence is not pertinent because there is no decay of the pulsation amplitude in time. Thus, the use of results of stability theory of Taylor-Culick flow to analyze a limit-cycle in SRMs is unwarranted. In other words, the experimentally observed time dependence of the pulsation amplitude in SRMs is actually a dependence on Mach number. In the experiments involving combustion of propellant, it becomes a time dependence because in these the inlet Mach number decreases in time. However, the stability theory remains fundamental for the study of the formation of vortices due to surface vortex shedding (SVS). 


\subsubsection{Two and three dimensional simulations and the effect of turbulence}

Flow at high Reynolds numbers display chaotic behavior induced by convective nonlinearity. Turbulence affects heat transport, bulk combustion and the structure of large scale vortices. A detailed description of the turbulent flow in SRMs by using a direct solution of the equations of motion would imply threedimensional numerical simulations with about $10^{9}$ grid points (Fabignon et al. 2016). To save on numerical cost it is appealing to perform two-dimensional or axis-symmetrical simulations with simplified turbulence models such as URANS or $\mathrm{LES}^{7}$. As the flow velocity ranges from very small near the head end to $M c$ at the nozzle inlet one encounters in the SRM the fundamental difficulty of the transition from laminar to turbulent flow, which is particularly difficult to model (Kuentzmann et al. 2004). The importance and indeed the exact role of turbulence on limit-cycle pulsation in SRMs remains uncertain.

Some authors use commercial codes such as FLUENT and ANSYS with and without turbulence models (Stella and Paglia 2009; Stella and Paglia 2011; Zhang et al. 2012; Waxing et al. 2013). Results obtained using CPS a in house ArianeGroup code indicate that the pulsation level is reduced by $30 \%$ to $50 \%$ when turbulence is modeled (Gallier et al. 2009). For experiments with nozzle cavity, using URANS, a fair prediction of pulsation level in LP9 scale-model experiments is found. Using LES, a large discrepancy between experiments and simulation is observed for the case without nozzle cavity. This is likely due to the fact that the pulsation amplitudes without cavities are too low to predict accurately. Indeed, when in the low-amplitude range pulsation amplitudes become extremely sensitive to small variations in set-up parameters (Bruggeman et al. 1991; Tonon et al. 2011).

\subsection{Research objectives}

The main objective of the research is to develop reduced-order analytical or semi-analytical models of self-sustained pressure pulsations in large SRMs, with focus on limit-cycle behavior (Fig. 1.5). No ready made analytical "plug and play" models that can predict limit cycles are available in the literature for vortex driven pulsations. Namely, new models will have to be formulated from the basics. In this text two strategies are explored: the nonlinear energy balance approach and the global lumped element model approach.

\subsubsection{Energy balance approach}

The "energy balance" approach assumes that the system has reached a stable limit cycle. The limit cycle is taken to be dominated by a single estimated

${ }^{7}$ LES simulations are three-dimensional (Piomelli et al. 2018). 
acoustic mode in its fundamental harmonic. I.e. a standing wave is assumed to exist allowing the user to estimate the amplitude. In first approximation, one can assume that the oscillation frequency $\omega$ is equal to the natural frequency $\omega_{n}$ of the mode. A problem with considering the energy balance in acoustics is that one should include all relevant second-order terms, which adds considerable complication (Culick 2006; Majdalani et al. 2006). However, a simplified model can be used for order of magnitude estimates (Hirschberg et al. 2016; Hirschberg et al. 2018), this will be demonstrated in chapter 5. Another problem is that the limit cycle oscillation frequency $\omega$ used in the energy balance is an input provided by the user. I.e. the model does not predict $\omega$. However, as calculation costs are quite low, the energy balance can easily be repeated for several acoustic modes. As such it can be viewed as a global engineering tool for order of magnitude prediction of pulsation amplitudes.

\subsubsection{Global lumped element model}

The formulation of a global lumped element model for the amplitudes of the acoustic modes, with direct combustion as a source, as suggested by Culick (Culick 2004; Culick 2006) is an established method in the literature. Such methods are commonly used to study the linear stability of SRM systems. The combination with nonlinear source terms for the prediction of limit cycles remains undeveloped. In this case, a set of coupled ordinary differential equations for the amplitude of acoustic modes as a function of time results. To obtain a limit cycle solution, one could use approximations for slow time evolution using e.g. the method of van der Pol (method of averaging) (Culick 2006). This allows for the prediction of the time evolution of amplitude and frequency of the oscillations, or even unstable limit cycle behavior. In the following text, a vortex sound analogy is derived (chapter 2). The analogy is then used to derive the lumped model for vortex driven oscillations, in chapter 2.

In particular the integral form of the vortex sound analogy will be used. In what follows a brief description of the necessary concepts is given. Using Green's theorem an integral equation of an aeroacoustic analogy can be constructed. The corresponding Green's function is the pulse response of the wave equation, and obeys causality conditions. When it satisfies the same linear boundary conditions as the physical problem of interest, it is referred to as a tailored Green's function (Ffowcs Williams and Howe 1975; Howe 2014). The integral formulation of the aeroacoustic analogy is the convolution integral over time and space of the aeroacoustic sound sources with the Green's function. When a tailored Green's function is used it combines the effect of sources, propagation, boundary conditions and initial conditions in a single formula. The integral representation is useful as it splits up the problem into a purely linear problem of finding a Green's function and a second problem where the integral equation is solved. The integral equation is also convenient when introducing approxima- 
tions, because integration tends to smooth out approximation errors (Crighton et al. 1992; Howe 2014; Rienstra and Hirschberg 2015).

\subsection{Thesis overview}

In chapter 2, a vortex sound analogy, for an acoustically closed-closed pipe, is derived. The integral form of this analogy is used to derive a harmonic oscillator equation for the pulsation amplitude due to vortex-nozzle interaction driven indirect sound. This equation can be used to formulate a global lumped element model for limit-cycle pulsations in large solid rocket motors. However, before this can occur a sound source model of vortex-nozzle interaction needs to be developed.

To this end, detailed investigations of vortex-nozzle interaction have been performed. A dedicated analytical incompressible two-dimensional plane model for vortex-nozzle interaction as a sound source is first derived in chapter 3. Specifically, in chapter 3 sound generation due to interaction of a line vortex with a subcritical contraction in an infinite channel is considered. Then, to study the influence of compressibility effects on vortex-nozzle interaction and a choked contraction, dedicated simulations were carried out using an Euler code for Internal Aeroacoustics (EIA). This study is described in chapter 4. A lumped element source model and scaling rules for the sound source is proposed. These calculations also provide information on the influence of the nozzle geometry on the sound source.

In chapter 5 , the formulation of an energy balance model for limit cycle pulsations is described. This model incorporates the incompressible vortex dynamics model developed in chapter 3. The results are compared to cold-gas scale experiments reported by Anthoine (Anthoine 2000). The energy balance model does not allow for the prediction of the variation of oscillation frequency with Mach number $M$, as observed in experiments and real flight (Dotson et al. 1997; Anthoine 2000; Fabignon et al. 2003; Anthoine et al. 2015). To include the variation of the oscillation frequency a result derived from the harmonic oscillator equation (global lumped model approach chapter 2) is used.

In order to compare the contributions of entropy and vortex driven indirect sound in large SRMs, systematic dedicated vortex-nozzle and entropy spotnozzle interaction simulations have also been performed. Interpretation of these results in combination with information learned from cold-gas scale model experiments (Anthoine 2000) and scale model experiments with combustion (Gallier et al. 2009), provides insight into the relative importance of these two indirect sound sources. The results of this study are described in chapter 6 . 
After chapter 6, global conclusions are drawn. These are followed by suggestions for further research. 


\title{
Chapter 2
}

\section{Global lumped element model for vortex sound in large Solid Rocket Motors}

\begin{abstract}
A detailed derivation of a harmonic oscillator equation for the pulsation amplitude using a vortex sound analogy is given. The analogy is similar to Howe's vortex sound analogy. However, it contains an extra source term, which proves essential for finding the correct acoustic radiation losses at the nozzle. When a volume integral in the analogy is neglected one recovers a result very similar to the expected results. The approximation is accurate both for axial and radial gas injection into a cylindrical combustion chamber. The accuracy of the approximation remains to be established for arbitrary chamber geometries and actual combustion conditions.
\end{abstract}

\subsection{Vortex sound analogy}

To derive the vortex sound analogy, the mass conservation law

$$
\frac{1}{\rho} \frac{\mathrm{D} \rho}{\mathrm{D} t}=-\nabla \cdot \mathbf{v}
$$

and (neglecting viscous friction because of the assumed high Reynolds number

This chapter is in based on the conference paper: L. Hirschberg, T. Schuller, C. Schram, J. Collinet, "Interaction of a vortex with a contraction in a 2-dimensional channel: incompressible flow prediction of sound pulse", 24th AIAA/CEAS Aeroacoustics Conference, AIAA AVIATION Forum (2018) 
of the flow) the Euler equations of motion

$$
\frac{\mathrm{D} \mathbf{v}}{\mathrm{D} t}=-\frac{\nabla p}{\rho}
$$

for a single phase flow are used. Here $\rho$ is the density, $\mathbf{v}$ is the velocity and $p$ the pressure. The operator $\mathrm{D} / \mathrm{D} t \equiv \partial / \partial t+\mathbf{v} \cdot \nabla \mathbf{v}$ is the material derivative. Substituting the identity

$$
\mathbf{v} \cdot \nabla \mathbf{v}=\nabla \frac{|\mathbf{v}|^{2}}{2}+\boldsymbol{\omega} \times \mathbf{v}
$$

in the Euler equations one obtains Crocco's equation:

$$
\frac{\partial \mathbf{v}}{\partial t}+\nabla \frac{|\mathbf{v}|^{2}}{2}+\frac{\nabla p}{\rho}=-\boldsymbol{\omega} \times \mathbf{v}
$$

where $\boldsymbol{\omega} \equiv \nabla \times \mathbf{v}$ is the vorticity. It is now assumed that the flow is homentropic and isentropic. As the Prandtl number of a gas flow is order unity, if one neglects viscous effects, heat transfer should be negligible as well. The flow is isentropic $\mathrm{D} s / \mathrm{D} t=0$. In a Taylor-Culick flow gas at temperature $T_{w}$ is injected at low velocity $V_{\text {inj }}$ at the wall. Due to accumulation of mass the main flow velocity grows linearly with the distance $x$ along the pipe axis, involving an adiabatic expansion. As the nozzle inlet is reached between the wall and the axis of the combustion chamber the relative temperature difference reaches the value:

$$
\frac{T_{w}-T}{T}=\frac{\gamma-1}{2} M^{2}
$$

here $\left(V_{\text {inj }} / c\right)^{2}$ has been neglected, $M$ is the nozzle inlet Mach number, and $\gamma=\frac{C_{p}}{C_{V}}$ is the ratio of the heat capacity at constant pressure and volume, respectively (Thompson 1972). For air as used in cold-gas scale models $\gamma=1.4$. As the inlet Mach number range is $0.05 \leq M \leq 0.2$, relative temperature differences due to flow are at most of the order $10^{-2}$. It is reasonable to assume an homentropic flow $(\nabla s=0)$ in cold-gas scale experiments and in cases involving combustion if one assumes combustion to be restricted to a thin region near the propellant wall. If combustion ${ }^{2}$ occurs outside this thin region, e.g. in traveling vortices as occurs in hybrid rocket motors (Carmincino 2009), this approximation might not apply. However, these cases are not considered here. I.e. for modeling purposes the flow in a SRM is assumed to behave as in a cold-gas scale experiment.

\footnotetext{
${ }^{2}$ For simulations involving combustion products Gallier and Godfroy use $\gamma=1.16$ (Gallier and Godfroy 2009).
} 
For such a isentropic-homentropic flow one has

$$
\frac{\nabla p}{\rho}=\nabla h
$$

where $h$ is the specific enthalpy defined by:

$$
h=e+\frac{p}{\rho}
$$

with $e$ the specific internal energy. Introducing the total specific enthalpy

$$
B=h+\frac{|\mathbf{v}|^{2}}{2}
$$

Crocco's equation (Eq. (2.4)) becomes

$$
\frac{\partial \mathbf{v}}{\partial t}+\nabla B=-\boldsymbol{\omega} \times \mathbf{v} .
$$

Taking the divergence of this equation one obtains:

$$
\nabla^{2} B=-\nabla \cdot(\boldsymbol{\omega} \times \mathbf{v})-\frac{\partial \nabla \cdot \mathbf{v}}{\partial t} .
$$

Using the conservation of mass (Eq. (2.1)) this becomes

$$
\nabla^{2} B=-\nabla \cdot(\boldsymbol{\omega} \times \mathbf{v})+\frac{\partial}{\partial t}\left(\frac{1}{\rho} \frac{\mathrm{D} \rho}{\mathrm{D} t}\right)
$$

The key idea is now is to derive a wave equation with source terms at the righthand side, which when the source terms vanish, describes the acoustic wave propagation at an observer position. Assuming this observer is in the combustion chamber near the head end wall we assume around this position an acoustic field described by the homogeneous wave equation of d'Alembert for a stagnant uniform fluid

$$
\frac{1}{c_{0}^{2}} \frac{\partial^{2} B^{\prime}}{\partial t^{2}}-\nabla^{2} B^{\prime}=0
$$

where $c_{0}$ is the time-averaged local speed of sound near the observer and the acoustic perturbation $B^{\prime}=B-B_{0}$ is defined relative to the time-averaged state around the observer where the total enthalpy has the average value $B_{0}$. 
Subtracting the factor $\frac{1}{c_{0}^{2}} \frac{\partial^{2} B^{\prime}}{\partial t^{2}}$ on both sides of Eq. 2.11 and defining the perturbations as deviations from the observer state, one obtains the analogy:

$$
\frac{1}{c_{0}^{2}} \frac{\partial^{2} B^{\prime}}{\partial t^{2}}-\nabla^{2} B^{\prime}=\nabla \cdot(\boldsymbol{\omega} \times \mathbf{v})+\frac{\partial}{\partial t}\left(\frac{1}{c_{0}^{2}} \frac{\partial^{2} B^{\prime}}{\partial t^{2}}-\frac{1}{\rho} \frac{\mathrm{D} \rho^{\prime}}{\mathrm{D} t}\right)
$$

where the perturbation $\rho^{\prime} \equiv \rho-\rho_{0}$ is defined with respect to the observer state average value of the density $\rho_{0}$. Note that $B_{0}, c_{0}$ and $\rho_{0}$ are constants. As the head end wall of the combustion chamber is solid one has a full acoustic reflection. This results in an acoustic field dominated by standing waves (composed of traveling longitudinal plane waves of opposite direction and equal amplitude). In such a case the convective effects during wave propagation in one direction are compensated by the convective effects while traveling in the opposite direction (Morfey 1971; Wang and Kassoy 1992). At this point Howe ${ }^{3}$ (Howe 1998) states without detailed argument that the only relevant term on the righthand side is $\nabla \cdot(\boldsymbol{\omega} \times \mathbf{v})$ and neglects all other source terms.

However, using Howe's approximation to construct a global lumped element model one finds that it neglects convective acoustic radiation losses at the nozzle. These are essential (Buffum et al. 1966; Culick 2006). In order to recover these loss terms the second term on the righthand side of Eq. 2.13 is kept but linearized with respect to the observer state. The following ad hoc approximations are used

$$
B^{\prime} \simeq \frac{p^{\prime}}{\rho_{0}}
$$

here convective effects are neglected. Using the isentropic hypothesis in linear approximation we have furthermore that

$$
\rho^{\prime}=\frac{p^{\prime}}{c_{0}^{2}}
$$

The convective term in $\frac{1}{\rho} \frac{\mathrm{D} \rho}{\mathrm{D} t}$ is linearized to find

$$
\frac{1}{\rho} \frac{\mathrm{D} \rho}{\mathrm{D} t}=\frac{1}{\rho_{0}}\left(\frac{\partial \rho^{\prime}}{\partial t}+\mathbf{U} \cdot \nabla \rho^{\prime}\right)
$$

where $\mathbf{U}$ is the time averaged flow velocity. Using this approximations one finds

$$
\frac{1}{c_{0}^{2}} \frac{\partial^{2} B^{\prime}}{\partial t^{2}}-\nabla^{2} B^{\prime}=\nabla \cdot(\boldsymbol{\omega} \times \mathbf{v})-\frac{1}{c_{0}^{2}} \frac{\partial}{\partial t}\left(\mathbf{U} \cdot \nabla B^{\prime}\right) .
$$

\footnotetext{
${ }^{3}$ Howe (Howe 1998) defines the acoustic velocity $\mathbf{u}^{\prime}$ using the linearized Euler equation $\frac{\partial \mathbf{u}^{\prime}}{\partial t}=-\nabla B^{\prime}$. This implies that $\mathbf{u}^{\prime}$ is a irrotational potential flow.
} 
Using these ad hoc approximations it will be shown that by using the integral formulation one can recover the correct nozzle convective radiation losses in a global lumped element model.

\subsection{Low Mach number approximation derivation of the pulsation amplitude harmonic oscillator equa- tion.}

To derive a global lumped model, the integral form of the vortex sound analogy (Eq. 2.17) is used, this involves the use of a Green's function. One can find a Green's function $G=G(\mathbf{x}, \mathbf{y}, t-\tau)$ such that

$$
\frac{1}{c_{0}^{2}} \frac{\partial^{2} G}{\partial t^{2}}-\nabla^{2} G=\delta(\mathbf{x}-\mathbf{y}) \delta(t-\tau)
$$

with suitable boundary and/or initial conditions. The Green's function has the following causality properties $G=0$ and $\partial G / \partial t=0$ for $t-\tau \leq 0$.

After Bruggeman et al. (Bruggeman et al. 1991) the Green's function for a ducted problem is taken to be

$$
G=\sum_{n}\left(\frac{c_{0}}{E_{n}}\right)^{2} \frac{\mathcal{H}(t-\tau) \psi_{n}(\mathbf{x}) \psi_{n}(\mathbf{y}) \sin \left(\omega_{n}(t-\tau)\right)}{\omega_{n}}
$$

where $\mathcal{H}(t-\tau)$ is a Heaviside function, $\psi_{n}$ is an acoustic mode of the system and $\omega_{n}$ is the resonance frequency corresponding to that mode. The mode $\psi_{n}(\mathbf{x})$ is a solution of the Helmholtz equation viz.

$$
\nabla^{2} \psi_{n}+k_{n}^{2} \psi_{n}=0
$$

where $k_{n}=\omega_{n} / c_{0}$. It is assumed that $k_{n} \neq k_{m}$ and either $\psi_{n}=0$ or $\mathbf{n} \cdot \nabla \psi_{n}=0$ on the various parts of surface $S$ enclosing $V$ (delimiting the system considered), where $\mathbf{n}$ is the outer normal on $S$. From the Helmholtz equation one can derive the well known orthogonality condition

$$
\int_{V} \psi_{n} \psi_{m} \mathrm{~d}^{3} x=E_{n}^{2} \delta_{n m},
$$

where $E_{n}^{2}=\int_{V} \psi_{n}^{2} \mathrm{~d}^{3} x$ is a normalization factor and $\delta_{n m}$ is a Kronecker delta $\left(\delta_{n m}=0\right.$ for $n \neq m$ and $\delta_{n m}=0$ for $\left.n=m\right)$. The derivation for this relation 
is given in appendix A.

The stagnation enthalpy fluctuation is also expanded in modes after Bruggeman et al. (Bruggeman et al. 1991), viz.

$$
B^{\prime}=\sum_{n} b_{n}(t) \psi_{n}(\mathbf{x})
$$

where $\psi_{n}(\mathbf{x})$ is the nth mode and $b_{n}(t)$ is the associated amplitude.

Multiplying Eq. (2.17) and Eq. (2.18) by $G$ and $B^{\prime}$ respectively and integrating over $\mathbf{y}$ and $\tau$, yields

$$
\begin{aligned}
\int_{-\Theta}^{\Theta} \int_{V}\left(\frac{G}{c_{0}^{2}} \frac{\partial^{2} B^{\prime}}{\partial \tau^{2}}-G \nabla_{\mathbf{y}}^{2} B^{\prime}\right) \mathrm{d}^{3} y \mathrm{~d} \tau & =\int_{-\Theta}^{\Theta} \int_{V} G \nabla_{\mathbf{y}} \cdot(\boldsymbol{\omega} \times \mathbf{v}) \mathrm{d}^{3} y \mathrm{~d} \tau \\
& -\int_{-\Theta}^{\Theta} \int_{V} G \frac{\mathbf{U}}{c_{0}^{2}} \cdot \nabla_{\mathbf{y}} \frac{\partial B^{\prime}}{\partial \tau} \mathrm{d}^{3} y \mathrm{~d} \tau \\
\int_{-\Theta}^{\Theta} \int_{V}\left(\frac{B^{\prime}}{c_{0}^{2}} \frac{\partial^{2} G}{\partial \tau^{2}}-B^{\prime} \nabla_{\mathbf{y}}^{2} G\right) \mathrm{d}^{3} y \mathrm{~d} \tau & =\int_{-\Theta}^{\Theta} \int_{V} B^{\prime} \delta(\mathbf{x}-\mathbf{y}) \delta(t-\tau) \mathrm{d}^{3} y \mathrm{~d} \tau \\
& =B^{\prime}(\mathbf{x}, t) .
\end{aligned}
$$

Subtracting Eq. (2.23) from Eq. (2.24) and rearranging the terms, the following is found

$$
\begin{aligned}
B^{\prime}(\mathbf{x}, t) & =\int_{-\Theta}^{\Theta} \int_{V}\left(\frac{B^{\prime}}{c_{0}^{2}} \frac{\partial^{2} G}{\partial \tau^{2}}-\frac{G}{c_{0}^{2}} \frac{\partial^{2} B^{\prime}}{\partial \tau^{2}}\right) \mathrm{d}^{3} y \mathrm{~d} \tau \\
& +\int_{-\Theta}^{\Theta} \int_{V}\left(G \nabla_{\mathbf{y}}^{2} B^{\prime}-B^{\prime} \nabla_{\mathbf{y}}^{2} G\right) \mathrm{d}^{3} y \mathrm{~d} \tau \\
& +\int_{-\Theta}^{\Theta} \int_{V} G \nabla_{\mathbf{y}} \cdot(\boldsymbol{\omega} \times \mathbf{v}) \mathrm{d}^{3} y \mathrm{~d} \tau \\
& -\int_{-\Theta}^{\Theta} \int_{V} G \frac{\mathbf{U}}{c_{0}^{2}} \cdot \nabla_{\mathbf{y}}\left(\frac{\partial B^{\prime}}{\partial \tau}\right) \mathrm{d}^{3} y \mathrm{~d} \tau .
\end{aligned}
$$

The integrals in this equation will be integrated by parts. To make the following step clearer the four integrals above are defined $I_{1}, I_{2}, I_{3}$ and $I_{4}$ respectively. Starting with $I_{1}$, after partial integration one finds 


$$
\begin{aligned}
I_{1} & =\frac{1}{c_{0}^{2}} \int_{V}\left[B^{\prime} \frac{\partial G}{\partial \tau}-G \frac{\partial B^{\prime}}{\partial \tau}\right]_{-\Theta}^{\Theta} \mathrm{d}^{3} y \\
& -\frac{1}{c_{0}^{2}} \int_{V} \int_{-\Theta}^{\Theta}\left(\frac{\partial G}{\partial \tau} \frac{\partial B^{\prime}}{\partial \tau}-\frac{\partial B^{\prime}}{\partial \tau} \frac{\partial G}{\partial \tau}\right) \mathrm{d} \tau \mathrm{d}^{3} y .
\end{aligned}
$$

The second integral obviously vanishes. The first integral vanishes by virtue of causality $G=0$ for $\Theta \geq t$, and when it is assumed for $\tau \rightarrow-\infty$ one has $B^{\prime}=0$. As both integrals vanish one finds $I_{1}=0$.

Partially integrating $I_{2}$, yields

$$
\begin{aligned}
I_{2} & =\int_{-\Theta}^{\Theta} \int_{S}\left(G \nabla_{\mathbf{y}} B^{\prime}-B^{\prime} \nabla_{\mathbf{y}} G\right) \cdot \mathbf{n} \mathrm{d}^{2} y \mathrm{~d} \tau \\
& -\int_{-\Theta}^{\Theta} \int_{V}\left(\nabla_{\mathbf{y}} G \cdot \nabla_{\mathbf{y}} B^{\prime}-\nabla_{\mathbf{y}} B^{\prime} \cdot \nabla_{\mathbf{y}} G\right) \mathrm{d}^{3} y \mathrm{~d} \tau
\end{aligned}
$$

The second integral in Eq. 2.27 obviously vanishes, thus

$$
I_{2}=\int_{-\Theta}^{\Theta} \int_{S}\left(G \nabla_{\mathbf{y}} B^{\prime}-B^{\prime} \nabla_{\mathbf{y}} G\right) \cdot \mathbf{n} \mathrm{d}^{2} y \mathrm{~d} \tau
$$

The factor $(\boldsymbol{\omega} \times \mathbf{v}) \cdot \mathbf{n}$ vanishes on the surface $S$ (this can either be because $\mathbf{v}=0$ or in case that injection $\mathbf{v}$ is parallel to $\mathbf{n}$ at $S$ ) of the control volume the partial integral of $I_{3}$ becomes

$$
I_{3}=-\int_{-\Theta}^{\Theta} \int_{V} \nabla_{\mathbf{y}} G \cdot(\boldsymbol{\omega} \times \mathbf{v}) \mathrm{d}^{3} y \mathrm{~d} \tau
$$

Assuming $\nabla \cdot \mathbf{U}=0$, corresponding to a low Mach number approximation $M^{2}<<1$ and an isentropic flow approximation $p^{\prime}=c_{0}^{2} \rho^{\prime}$, one finds

$$
I_{4}=\int_{-\Theta}^{\Theta} \int_{V} \nabla_{\mathbf{y}} G \cdot \frac{\mathbf{U}}{c_{0}^{2}} \frac{\partial B^{\prime}}{\partial \tau} \mathrm{d}^{3} y \mathrm{~d} \tau-\int_{-\Theta}^{\Theta} \int_{S} \frac{G(\mathbf{U} \cdot \mathbf{n})}{c_{0}^{2}} \frac{\partial B^{\prime}}{\partial \tau} \mathrm{d}^{2} y \mathrm{~d} \tau .
$$

The second integral corresponds to energy losses by convection at the boundaries. The first integral will be neglected. The validity of this approximation for a cylindrical combustion chamber is discussed in appendix C (for complex geometries, the validity of this approximation remains to be verified). This yields 


$$
\begin{aligned}
B^{\prime}(\mathbf{x}, t) & \simeq \int_{-\Theta}^{\Theta} \int_{S}\left(G \nabla_{\mathbf{y}} B^{\prime}-B^{\prime} \nabla_{\mathbf{y}} G\right) \cdot \mathbf{n} \mathrm{d}^{2} y \mathrm{~d} \tau \\
& -\int_{-\Theta}^{\Theta} \int_{V} \nabla_{\mathbf{y}} G \cdot(\boldsymbol{\omega} \times \mathbf{v}) \mathrm{d}^{3} y \mathrm{~d} \tau \\
& -\int_{-\Theta}^{\Theta} \int_{S} G \frac{(\mathbf{U} \cdot \mathbf{n})}{c_{0}^{2}} \frac{\partial B^{\prime}}{\partial \tau} \mathrm{d}^{2} y \mathrm{~d} \tau .
\end{aligned}
$$

In the following description algebraic details of the steps taken are omitted, details on these can be found in appendix B.

Eq. 2.19 is used for the Green's function G. Multiplying Eq. (2.25) by $\psi_{n}(\mathbf{x})$ and integrating over $V$ yields, using the orthogonality condition (Eq. (2.21)), an equation for the amplitude $b_{n}=b_{n}(t)$ of the $n^{\text {th }}$ mode viz.

$$
\begin{aligned}
b_{n}(t) & =\left(\frac{c_{0}}{E_{n}}\right)^{2} \int_{-\Theta}^{\Theta} \int_{S}\left[\mathcal{H}(t-\tau) \frac{\psi_{n}(\mathbf{y}) \sin \left(\omega_{n}(t-\tau)\right)}{\omega_{n}} \nabla_{\mathbf{y}} B^{\prime}\right] \cdot \mathbf{n} \mathrm{d}^{2} y \mathrm{~d} \tau \\
& -\left(\frac{c_{0}}{E_{n}}\right)^{2} \int_{-\Theta}^{\Theta} \int_{V} \mathcal{H}(t-\tau) \frac{\nabla_{\mathbf{y}} \psi_{n}(\mathbf{y}) \sin \left(\omega_{n}(t-\tau)\right)}{\omega_{n}} \cdot(\boldsymbol{\omega} \times \mathbf{v}) \mathrm{d}^{3} y \mathrm{~d} \tau \\
& -\left(\frac{c_{0}}{E_{n}}\right)^{2} \int_{-\Theta}^{\Theta} \int_{S} \mathcal{H}(t-\tau) \frac{\psi_{n}(\mathbf{y}) \sin \left(\omega_{n}(t-\tau)\right)}{\omega_{n}}\left(\frac{\mathbf{U} \cdot \mathbf{n}}{c_{0}^{2}} \frac{\partial B^{\prime}}{\partial \tau}\right) \mathrm{d}^{2} y \mathrm{~d} \tau .
\end{aligned}
$$

To obtain the above result, the boundary condition $\mathbf{n} \cdot \nabla_{\mathbf{y}} \psi_{n}(\mathbf{y})=0$ on $S$ was used. The equation above can be simplified by taking the partial derivative with respect to time of Eq. (2.32), and using the fact that under integration $\delta(t-\tau) \sin \left(\omega_{n}(t-\tau)\right)$ terms vanish. Taking the second partial derivative of the stagnation enthalpy fluctuation $B^{\prime}$ with respect to time and recognizing that three of the resulting sums equal $\sum_{n}-\omega_{n}^{2} b_{n} \psi_{n}(\mathbf{x})$, one then finds

$$
\begin{aligned}
& \sum_{n}\left(\frac{c_{0}}{E_{n}}\right)^{2} \psi_{n}(\mathbf{x})\left(\frac{\mathrm{d}^{2} b_{n}}{\mathrm{~d} t^{2}}+\omega_{n}^{2} b_{n}\right)= \\
+ & \sum_{n}\left(\frac{c_{0}}{E_{n}}\right)^{2} \psi_{n}(\mathbf{x}) \int_{S}\left(\psi_{n}(\mathbf{y}) \nabla_{\mathbf{y}} B^{\prime}-B^{\prime} \nabla_{\mathbf{y}} \psi_{n}(\mathbf{y})\right) \cdot \mathbf{n} \mathrm{d}^{2} y \\
- & \sum_{n}\left(\frac{c_{0}}{E_{n}}\right)^{2} \psi_{n}(\mathbf{x}) \int_{V}(\boldsymbol{\omega} \times \mathbf{v}) \cdot \nabla_{\mathbf{y}} \psi_{n}(\mathbf{y}) \mathrm{d}^{3} y \\
- & \sum_{n}\left(\frac{c_{0}}{E_{n}}\right)^{2} \psi_{n}(\mathbf{x}) \int_{S} \psi_{n}(\mathbf{y}) \frac{\mathbf{U} \cdot \mathbf{n}}{c_{0}^{2}} \frac{\partial B^{\prime}}{\partial \tau} \mathrm{d}^{2} y
\end{aligned}
$$


Chapter 2 - Global lumped element model for vortex sound in large SOLID ROCKET MOTORS

defining $I_{n}^{(s)}, I_{n}^{(v)}$ and $I_{n}^{(c)}$ respectively, as follows ${ }^{4}$

$$
\begin{aligned}
I_{n}^{(s)} & =\left(\frac{c_{0}}{E_{n}}\right)^{2} \int_{S}\left(\psi_{n}(\mathbf{y}) \nabla_{\mathbf{y}} B^{\prime}-B^{\prime} \nabla_{\mathbf{y}} \psi_{n}(\mathbf{y})\right) \cdot \mathbf{n d}^{2} y \\
I_{n}^{(v)} & =-\left(\frac{c_{0}}{E_{n}}\right)^{2} \int_{V}(\boldsymbol{\omega} \times \mathbf{v}) \cdot \nabla_{\mathbf{y}} \psi_{n}(\mathbf{y}) \mathrm{d}^{3} y \\
I_{n}^{(c)} & =-\left(\frac{c_{0}}{E_{n}}\right)^{2} \int_{S} \psi_{n}(\mathbf{y}) \frac{\mathbf{U} \cdot \mathbf{n}}{c_{0}^{2}} \frac{\partial B^{\prime}}{\partial t} \mathrm{~d}^{2} y
\end{aligned}
$$

If one choses the boundary condition $\nabla_{\mathbf{y}} \psi_{n}(\mathbf{y}) \cdot \mathbf{n}=0$ at the head end and the nozzle (closed-closed pipe approximation) $I_{n}^{(s)}$ becomes

$$
I_{n}^{(s)}=\left(\frac{c_{0}}{E_{n}}\right)^{2} \int_{S} \psi_{n}(\mathbf{y}) \nabla_{\mathbf{y}} B^{\prime} \cdot \mathbf{n} \mathrm{d}^{2} y .
$$

Multiplying by $\psi_{n}(\mathbf{x})$ and integrating over $V$, Eq. (2.33) takes the form

$$
\frac{\mathrm{d}^{2} b_{n}}{\mathrm{~d} t^{2}}+\omega_{n}^{2} b_{n}=I_{n}^{(s)}+I_{n}^{(v)}+I_{n}^{(c)} .
$$

Hence the integral equation (2.31) has been replaced by a set of ordinary differential equations for the amplitudes $b_{n}$ of the modes $\psi_{n}(\mathbf{x})$. Each of these equations describes a harmonic oscillator. These oscillators are coupled through the phase condition for the vortex shedding and the non-linearity of source terms. When reaching a limit-cycle with period of oscillation $T$, the oscillation frequency of all the modes are either identical to $2 \pi / T$ or multiple of a fundamental frequency $2 \pi / T$.

Following the quasi-steady theory of Marble and Candel (Marble and Candel 1977), one has at the nozzle inlet (using Eq. (2.9) with $\boldsymbol{\omega}=0$ )

$$
\begin{aligned}
\nabla_{\mathbf{y}} B^{\prime} \cdot \mathbf{n} & =-\frac{\partial \mathbf{u}_{a c}}{\partial t} \cdot \mathbf{n} \\
& =-\frac{\gamma-1}{2} \frac{M}{\rho_{0} c_{0}} \frac{\partial p^{\prime}}{\partial t} \\
& \simeq-\frac{\gamma-1}{2} \frac{M}{c_{0}} \frac{\partial B^{\prime}}{\partial t}
\end{aligned}
$$

which can be substituted in $I_{n}^{(s)}$ (Eq. (2.34)) on the inlet surface of the nozzle. Combined with the convective term $I_{n}^{(c)}$, one finds the nozzle radiation losses as

\footnotetext{
${ }^{4}$ The boundary condition for $\nabla_{\mathbf{y}} B^{\prime} \cdot \mathbf{n}$ is obtained using the equation of Euler: $\nabla_{\mathbf{y}} B^{\prime} \cdot \mathbf{n}=$ $-\frac{\partial \mathbf{v}^{\prime} \cdot \mathbf{n}}{\partial t}$. For local boundary conditions $\mathbf{v}^{\prime} \cdot \mathbf{n}$ is related to the fluctuations in $B$ by an impedance $Z(\omega)$ in the frequency domain. In principle this a convolution in the time domain.
} 
calculated by Culick ${ }^{5}$ (Buffum et al. 1966; Culick 2006) including convection effects:

$$
I_{n}^{(s)}+I_{n}^{(c)}=-\left(\frac{c_{0}}{E_{n}}\right)^{2} \int_{S_{\text {nozzle }}} \psi_{n}(\mathbf{y}) \frac{\gamma+1}{2} \frac{M}{c_{0}} \frac{\partial B^{\prime}}{\partial t} \mathrm{~d}^{2} y
$$

\subsection{Conclusion}

A derivation of a lumped model for vortex driven pressure pulsations is provided. To derive this lumped model a vortex sound analogy is employed. The analogy is similar to Howe's vortex sound analogy. However, it differs due to the inclusion of an extra source term. This extra term proves essential to recover the expression for radiative acoustic losses at the nozzle including convection losses (Buffum et al. 1966; Culick 2006). During the derivation a term is neglected (Eq. (2.30)). This is justified for the case of a simplified cylindrical tube geometry. For a cylindrical combustion chamber, this approximation is accurate both for axial and radial gas injection into the combustion chamber. For an arbitrary combustion chamber geometry with actual combustion, the accuracy of the approximation remains to be established.

${ }^{5}$ Eq. (6.184) on page 6-49 in (Culick 2006) 


\title{
Chapter 3
}

\section{Analytical incompressible frictionless two-dimensional planar model for vortex-nozzle interaction}

\begin{abstract}
Sound generation by a vortex ingested by the nozzle is modelled by considering the interaction of a line vortex with a contraction in a channel. The flow is described by two-dimensional incompressible potential flow theory. Using conformal mapping, an analytical solution is found for the potential describing the base flow and the convection velocity of the vortex. The vortex path is obtained numerically, using a $4^{\text {th }}$ order Runge-Kutta integration method. A low-frequency and low Mach number approximation of vortex sound theory is used to deduce the sound wave generated by a vortex interacting with a contraction in an infinitely long channel. Results are compared to results obtained in the literature using either a compressible frictionless flow model or an incompressible model in which a discrete panel describes the interaction between the vortex and the wall. The sound appears to be mainly produced in the approach phase, where the flow is essentially subsonic. This explains why a low Mach number incompressible flow approximation is meaningful. A conformal mapping is then found to map a channel with a contraction with rounded edge onto a plane wall. This allows the effect of the edge shape on sound production to be explored. While the amplitude of the acoustic response pulse is reduced by rounding off the edge, the time integral of the pulse appears to be only weakly dependent on the edge shape. Furthermore, this integral is not sensitive to the contraction ratio and to the height at which the vortex approaches the nozzle, but is proportional to the square of the vortex circulation. The model can be used as a test case for more complex numerical models and provides some
\end{abstract}


insight into the influence of parameters on the sound production.

\subsection{Introduction}

In the absence of nozzle cavity the pulsation level in a SRM is much lower (Anthoine 2000; Gallier et al. 2009). In the present chapter, the acoustic pulse generated by a vortex passing through a sudden contraction in an infinitely long channel, without the presence of a nozzle cavity, is considered. Hulshoff et al. (Hulshoff et al. 2001) considered this interaction in a two-dimensional frictionless compressible flow. They demonstrated that the sound pulse generated by ingestion of a vortex by a choked nozzle can reasonably well be predicted by considering a point vortex in an incompressible potential flow. The sound production in the limit of vanishing Mach number is described by a low-frequency approximation of the vortex sound theory proposed by Howe (Howe 2014). The interaction of the point vortex with the walls was described by using a discrete panel method (Hulshoff et al. 2001).

The present chapter considers a conformal mapping method, which yields an analytical solution for the flow field. This allows for a systematic study of the influence of parameters such as the nozzle inlet shape.

Using a conformal mapping proposed by Henrici (Henrici 1974) one can study the effect of rounding off the edge of a sudden contraction in the channel geometry. One can furthermore study the effect of the ratios $S_{1} / S_{2}$ of a channel cross-section up and down stream of the contraction. The effect of vortex circulation $\Gamma$ and height $h$ at which it approaches the contraction can be studied as well.

The vortex sound theory of Howe (Howe 2014) uses the total enthalpy fluctuations $B^{\prime}$ as the aeroacoustic variable. This allows convective effects in the boundary conditions to be taken into account while neglecting these effects in the wave propagation (Nicoud and Wieczorek 2009). This approximation neglects second order terms $M^{2}$ in the Mach number $M=U / c$ of the main flow $U$ (with $c$ the speed of sound). Results of Hulshoff et al. (Hulshoff et al. 2001) indicate that this is a reasonable assumption for the conditions of interest.

The following sections describe the components of the model: the potential flow theory used is described in section 3.2, the conformal mapping method is described in section 3.3. This mapping transforms the channel in physical plane

This chapter is in based on a conference paper: L. Hirschberg, T. Schuller, C. Schram, J. Collinet, M. Yiao, A. Hirschberg. "Interaction of a vortex with a contraction in a twodimensional channel: incompressible flow prediction of sound pulse", 23rd AIAA/CEAS Aeroacoustics Conference, AIAA AVIATION Forum, (AIAA 2017-3701) 
Chapter 3 - AnAlytical incompressible frictionless tWo-Dimensional

PLANAR MODEL FOR VORTEX-NOZZLE INTERACTION

$z=x+\mathrm{i} y$ into the half infinite space in the $\zeta$-plane. In the $\zeta$-plane analytical expression for the flow potential and flow velocity are obtained. In section 3.4 the path of a line vortex with circulation $\Gamma$ is considered. This path is obtained by time integration using a $4^{\text {th }}$ order Runge-Kutta method (Howe 2014).

The transformation from the $\zeta$-plane back to the physical $z$-plane is explicit. Therefore the time integration is carried out in the $\zeta$-plane. The integration method is first tested by considering the flow without a vortex. As an additional test, the case of a line vortex passing around the edge of a semi-infinite plane is used, for which there is an analytical solution (Crighton 1972). Section 3.5 provides a summary of the vortex sound theory. Section 3.6 discusses a method for the prediction of the sound radiation using a low-frequency Green's function in which one assumes the channel to be infinitely long (Howe 2014). In section 3.7 results are discussed. Section 3.8 provides conclusions.

\subsection{Potential theory}

Consider a two dimensional flow with velocity $\mathbf{v}=(u, v, 0)$ in the physical z-plane $(z=x+\mathrm{i} y)$ with complex potential (Prandtl and Tiejens 1934):

$$
F(z)=\Phi(x, y)+\mathrm{i} \Psi(x, y)
$$

where the scalar potential $\Phi$ is defined, such that:

$$
\begin{aligned}
& u=\frac{\partial \Phi}{\partial x}, \\
& v=\frac{\partial \Phi}{\partial y} .
\end{aligned}
$$

And the stream function $\Psi$ is defined, such that:

$$
\begin{aligned}
& u=\frac{\partial \Psi}{\partial y} \\
& v=-\frac{\partial \Psi}{\partial x} .
\end{aligned}
$$

The variation $d F$ in $F$ is given by:

$$
\mathrm{d} F=\frac{\partial \Phi}{\partial x} \mathrm{~d} x+\frac{\partial \Phi}{\partial y} \mathrm{~d} y+\mathrm{i}\left(\frac{\partial \Psi}{\partial x} \mathrm{~d} x+\frac{\partial \Psi}{\partial y} \mathrm{~d} y\right)=(u-\mathrm{i} v)(\mathrm{d} x+\mathrm{i} \mathrm{d} y)=V^{*} \mathrm{~d} z
$$

where $V=u+\mathrm{i} v$. Hence $\mathrm{d} F / \mathrm{d} z=V^{*}$.

The potential $F_{Q}$ for a volume line source of strength $\mathrm{Q}$ placed at position $z_{Q}$ in an unbounded space is:

$$
F_{Q}=\frac{Q}{2 \pi} \ln \left(z-z_{Q}\right)
$$


The potential $F_{\Gamma}$ for a line vortex of circulation $\Gamma$ placed at position $z_{\Gamma}$ in an unbounded space is:

$$
F_{\Gamma}=-\mathrm{i} \frac{\Gamma}{2 \pi} \ln \left(z-z_{\Gamma}\right) .
$$

For the sake of brevity, we will henceforth refer to the line source and line vortex as source and vortex.

\subsection{Schwartz-Christoffel transformation}

Consider in the complex plane $z=x+\mathrm{i} y$, a wedge delimited between two halfinfinite lines joining at point $z_{1}$ with an exterior angle $\alpha_{1}$ of the vertex. The angle is between vectors along the two wedge boundaries, in directions that one follows walking along the path on top of the z-plane, keeping the inner (relevant) domain of the wedge at the left hand. The conformal mapping

$$
z=K\left(\zeta-\zeta_{1}\right)^{1-\frac{\alpha_{1}}{\pi}}
$$

maps (for a specific choice of the constant $K$ ) the interior of the wedge onto the upper half plane in the $\zeta$-plane with $\zeta_{1}$ the image of the vertex $z_{1}$. The position $\zeta_{1}$ can be chosen arbitrarily on the real axis. In differential form, this transformation is

$$
\frac{\mathrm{d} z}{\mathrm{~d} \zeta}=\frac{K^{\prime}}{\left(\zeta-\zeta_{1}\right)^{\frac{\alpha_{1}}{\pi}}}
$$

This transformation is conformal everywhere except at $z_{1}$. Its singularity at $z_{1}$ allows the vertex boundaries to be bent onto a straight line in the $\zeta$-plane. One can map the interior of a polygon with $\mathrm{N}$ vertices $z_{i}(i=1,2,3 \ldots N)$ in the z-plane, onto the upper $\zeta$-plane by using the so called Schwartz-Christoffel transformation

$$
\frac{\mathrm{d} z}{\mathrm{~d} \zeta}=K^{\prime} \Pi_{i=1}^{N}\left(\zeta-\zeta_{i}\right)^{-\frac{\alpha_{i}}{\pi}}
$$

The position of the images of three vertices can be chosen arbitrarily on the real axis of the $\zeta$-plane, as long as the sequence of positions (along the continuous path around the domain boundaries keeping the domain on the lefthand side) is respected.

In the following, the mapping of an abrupt contraction and a contraction with a rounded off edge are briefly discussed. A more detailed derivation is provided 
Chapter 3 - AnAlytical incompressible frictionless tWo-Dimensional

PLANAR MODEL FOR VORTEX-NOZZLE INTERACTION

in appendix D.

The mapping of an abrupt contraction at $x=0$ from a channel height $S_{1}$ $(x>0)$ to a channel height $S_{2}(x<0)$ is described by the transformation (Milne-Thomson 1968; Brown and Churchill 2009)

$$
\frac{\mathrm{d} z}{\mathrm{~d} \zeta}=K^{\prime} \zeta^{-1}(\zeta-1)^{1 / 2}(\zeta-a)^{-1 / 2}
$$

where $K^{\prime}=S_{1} / \pi$ and $a=\left(S_{1} / S_{2}\right)^{2}$. The first vertex at $z \rightarrow-\infty$ is mapped at $\zeta=0$. The second vertex at $z=\mathrm{i}\left(S_{1}-S_{2}\right)$ is mapped onto $\zeta=1$. The third vertex at $z=0$ is mapped onto $\zeta=a$. The integral version of this transformation is (Milne-Thomson 1968; Brown and Churchill 2009)

$$
z=\frac{S_{1}}{\pi}\left[\ln \frac{1+\tau}{1-\tau}-\frac{1}{b} \ln \frac{b+\tau}{b-\tau}\right]
$$

with:

$$
b=\frac{S_{1}}{S_{2}}=\sqrt{a}
$$

and

$$
\tau=\sqrt{\frac{\zeta-a}{\zeta-1}} .
$$

The square edge at $z=\mathrm{i}\left(S_{1}-S_{2}\right)$ can be replaced by a gradual transition over a distance $d$ (from $z=0$ to $z=-d+\mathrm{i}\left(S_{1}-S_{2}\right)$ ) (see Fig. 3.1) by replacing the factor $K^{\prime}(\zeta-1)^{1 / 2}$ in the transformation (equation (3.12)) by a factor $\left[\alpha(\zeta-1)^{1 / 2}+\beta(\zeta-1)^{1 / 2}\right]$ as proposed by Henrici (Henrici 1974):

$$
\frac{\mathrm{d} z}{\mathrm{~d} \zeta}=\zeta^{-1}\left[\alpha(\zeta-1)^{1 / 2}+\beta(\zeta-a)^{1 / 2}\right](\zeta-a)^{-1 / 2} .
$$

The transformation becomes in integral form:

$$
z=\alpha\left[\ln \frac{1+\tau}{1-\tau}-\frac{1}{b} \ln \frac{b+\tau}{b-\tau}\right]+\beta \ln \left[\frac{\zeta}{a}\right]
$$

where the integration constant has been found by assuming that $z=0$ is mapped onto $\zeta=a=b^{2}$ (implying $\tau=0$ ). The constants $\alpha$ and $\beta$ are found 


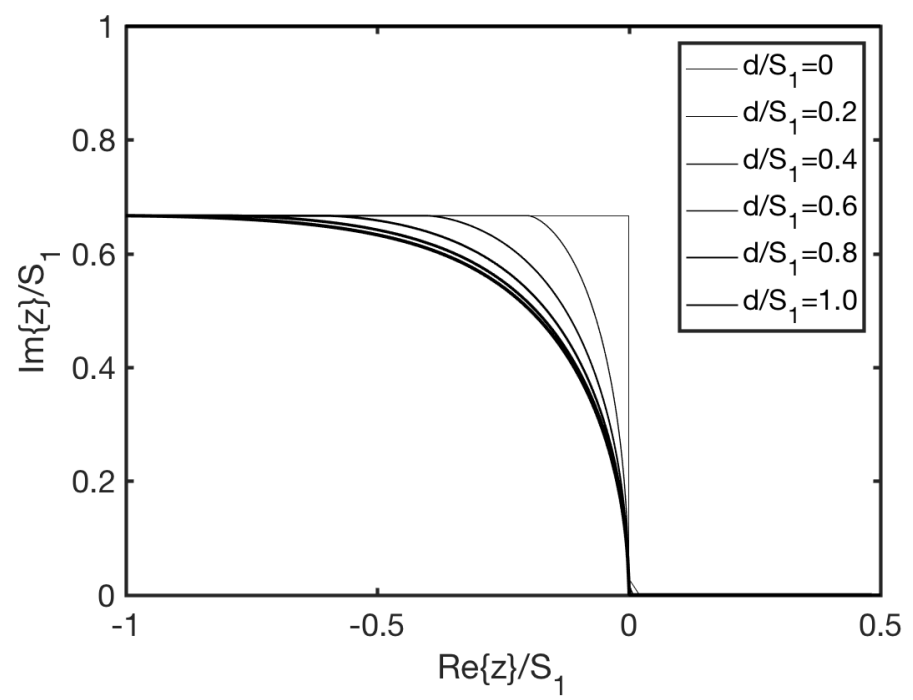

Figure 3.1: Geometry of channel with smooth contraction. The parameter $d$ can be considered as the length of the nozzle inlet. Geometries for various $d / S_{1}$ are shown. For $d / S_{1}=0$ one recovers the abrupt contraction, as $d / S_{1}$ is increased it an asymptotic solution is approached, which resembles the result for $d / S_{1}=1.0$.

by considering a flow (with potential $F=-\left(U S_{1} / \pi\right) \ln \zeta$ ) through the channel induced by a point source at $\zeta=0$ (see appendix D for more details), one finds

$$
\begin{gathered}
\alpha=\frac{S_{1}-S_{2}}{\pi}\left(\frac{b}{b-1}\right) \\
\beta=\frac{b S_{2}-S_{1}}{\pi(b-1)}
\end{gathered}
$$

The constant $b$ in equation (3.16) is found by substituting $\tau \rightarrow \infty$. This corresponds with $\zeta=1$ and $z=-d+\mathrm{i}\left(S_{1}-S_{2}\right)$ i.e. equation (3.16) becomes

$$
\begin{aligned}
-d+\mathrm{i}\left(S_{1}-S_{2}\right) & =\alpha \frac{S_{1}}{\pi b} \ln (-1)\left(1-\frac{1}{b}\right)-\beta \ln b^{2} \\
& =-2 \beta \ln b+\mathrm{i} \pi \alpha \frac{S_{1}}{\pi b}\left(1-\frac{1}{b}\right)
\end{aligned}
$$

Using equation (3.19) the real part of this result becomes

$$
\begin{aligned}
d & =2 \beta \ln b \\
& =2 \frac{b S_{2}-S_{1}}{\pi(b-1)} \ln b .
\end{aligned}
$$

One then finds an implicit equation for $b$ :

$$
b=\frac{S_{1}}{S_{2}}\left(1+\frac{\pi d}{2 S_{1} \ln b}(b-1)\right)
$$


The equation above can be solved by successive substitution, using the sharpedge $(d=0)$ limit, and $b=S_{1} / S_{2}$ as initial value for small values of $\pi d /\left(2 S_{2}\right)<$ 2 . For large values of $\pi d /\left(2 S_{2}\right)>2$ one can use $b=\exp \left(\pi d /\left(2 S_{2}\right)\right)$ as an initial value and solve equation (3.24) in the form

$$
b=\exp \left(\frac{\pi d}{2 S_{2}} \frac{1-\frac{1}{b}}{1-\frac{S_{1}}{S_{2} b}}\right)
$$

using successive substitution.

\subsection{Vortex path}

The main flow is induced by a $\operatorname{sink} Q=-S_{1} U$ at $\zeta=0$. The vortex with a circulation $\Gamma$ is at $\zeta_{\Gamma}$. This results in a potential:

$$
\mathcal{F}(\zeta)=-\frac{S_{1} U}{\pi} \ln (\zeta)-\mathrm{i} \frac{\Gamma}{2 \pi} \ln \left(\frac{\zeta-\zeta_{\Gamma}}{\zeta-\zeta_{\Gamma}^{*}}\right)
$$

where $\zeta_{\Gamma}^{*}$ is the position of the image of the vortex in the real axis of the $\zeta$-plane. The magnitude of the velocity of the flow far upstream of the contraction is $U$. The vortex is convected in the z-plane with the local flow velocity $V_{\Gamma}$ which is calculated from the derivative of the potential $F(z)$, from which the potential of the vortex is subtracted (avoiding self-induced velocity)

$$
V_{\Gamma}^{*}=\lim _{z \rightarrow z_{\Gamma}} \frac{\mathrm{d} \zeta}{\mathrm{d} z}\left(-\frac{S_{1} U}{\pi \zeta}+\mathrm{i} \frac{\Gamma}{2 \pi\left(\zeta-\zeta_{\Gamma}^{*}\right)}\right)-\mathrm{i} \frac{\Gamma}{2 \pi} \frac{\mathrm{d}}{\mathrm{d} z}\left(\ln \frac{\zeta-\zeta_{\Gamma}}{z-z_{\Gamma}}\right)
$$

Expanding the mapping $\zeta=f(z)$ in a Taylor series ${ }^{2}$ around $\zeta=\zeta_{\Gamma}$ one obtains the Routh correction (Clements 1973; Howe 2014):

$$
V_{\Gamma}^{*}=\left(\frac{\mathrm{d} \zeta}{\mathrm{d} z}\right)_{\zeta_{\Gamma}}\left(-\frac{S_{1} U}{\pi \zeta_{\Gamma}}+\mathrm{i} \frac{\Gamma}{2 \pi\left(\zeta_{\Gamma}-\zeta_{\Gamma}^{*}\right)}\right)-\mathrm{i} \frac{\Gamma}{4 \pi} \frac{\left(\frac{\mathrm{d}^{2} f}{\mathrm{~d} z^{2}}\right)_{z_{\Gamma}}}{\left(\frac{\mathrm{d} f}{\mathrm{~d} z}\right)_{z_{\Gamma}}^{2}}
$$

which corresponds in the $\zeta$-plane to a vortex convection velocity:

$$
\frac{\mathrm{d} \zeta_{\Gamma}}{\mathrm{d} t}=\left[V_{\Gamma}^{*}\right]^{*} \frac{1}{\left(\frac{\mathrm{d} z}{\mathrm{~d} \zeta}\right)_{\zeta_{\Gamma}}}=\frac{1}{\left|\frac{\mathrm{d} z}{\mathrm{~d} \zeta}\right|_{\zeta_{\Gamma}}^{2}}\left[-\frac{S_{1} U}{\pi \zeta_{\Gamma}}+\mathrm{i} \frac{\Gamma}{2 \pi\left(\zeta_{\Gamma}-\zeta_{\Gamma}^{*}\right)}+\mathrm{i} \frac{\Gamma}{4 \pi} \frac{\left(\frac{\mathrm{d}^{2} z}{\mathrm{~d} \zeta^{2}}\right)_{\zeta_{\Gamma}}}{\left(\frac{\mathrm{d} z}{d \zeta}\right)_{\zeta_{\Gamma}}}\right]^{*}
$$

\footnotetext{
${ }^{2}$ As $z \rightarrow z$ the factor $\left(\zeta-\zeta_{\Gamma}\right) /\left(z-z_{\Gamma}\right)$ will be undetermined for $\zeta=\zeta_{\Gamma}$. l'Hôpital's rule is needed to solve this problem.
} 
Where $\mathrm{d} z / \mathrm{d} \zeta$ is an explicit function of $\zeta$ (equation (3.16)), from which $\mathrm{d}^{2} z / \mathrm{d} \zeta^{2}$ can be found analytically for given $\zeta$.

A detailed derivation of this equation is presented in appendix E. A $4^{\text {th }}$ order Runge-Kutta integration scheme is used to calculate $\zeta_{\Gamma}(t)$ while the vortex position $z_{\Gamma}(t)$ is calculated using the explicit transformation given by equation (3.17).

As a first test case for the integration procedure we compared the streamline $\arg (\zeta)=\pi h / S_{1}$ with the streamline obtained by time integration. In these tests the parameter $h$ corresponds to the height above the bottom of the channel at which the vortex has been released. As a second test case we compared the path of a vortex around the edge of a semi-infinite plate with the analytical expression obtained by Crighton (Crighton 1972). In both cases, the difference between numerical integration and the analytical expression was of the order of the machine accuracy $\left(10^{-15}\right)$.

\subsection{Vortex sound theory: Howe's approximation}

As discussed in chapter 2. The sound production of a vortical flow at low Mach numbers is most efficiently predicted by using the so-called vortex sound theory (Howe 2014). This is a formal solution of a non-homogeneous wave equation, derived from the mass conservation law and the momentum equation respectively. Howe proposes the following analogy (chapter 2) (Howe 1998):

$$
\frac{1}{c_{0}^{2}} \frac{\partial^{2} B^{\prime}}{\partial t^{2}}-\nabla^{2} B^{\prime}=\nabla \cdot(\boldsymbol{\omega} \times \mathbf{v})
$$

In the next section an equivalent integral equation is provided in terms of a Tailored Green's function. Note that this is not the same vortex sound analogy as was used in chapter 2. Indeed, Eq. (2.17) is the vortex sound analogy used to formulate the global lumped element model for an acoustically closed-closed pipe. Here Eq. (3.30) will be used to find sound production due to the interaction of a line vortex with a contraction in an infinite duct.

\subsection{Sound generation}

A key step in the use of the analogy is to formulate the solution of Eq. (3.30) as a convolution of the source $\nabla \cdot(\boldsymbol{\omega} \times \mathbf{v})$ with the acoustic response of the system to a pulse in time generated at time $\tau$ by a point source placed at position $\mathbf{y}=\left(y_{1}, y_{2}, y_{3}\right)$, as observed at time $t$ by a listener at position $\mathbf{x}=\left(x_{1}, x_{2}, x_{3}\right)$. 
Chapter 3 - AnAlytical incompressible frictionless tWo-Dimensional

PLANAR MODEL FOR VORTEX-NOZZLE INTERACTION

The $x_{1}$ direction is along the channel axis in the direction of the segment with cross section $S_{1}$. This is the so-called Green's function $G(\mathbf{x}, \mathbf{y},(t-\tau))$ or the solution of the equation:

$$
\frac{1}{c_{0}^{2}} \frac{\partial^{2} G}{\partial t^{2}}-\nabla^{2} G=\delta(\mathbf{x}-\mathbf{y}) \delta(t-\tau)
$$

for initial and boundary conditions which are chosen by the "user". This Green's function does not depend on $t$ and $\tau$ individually. It depends on the difference $t-\tau$. Furthermore, the reciprocity principle $G(\mathbf{x}, \mathbf{y}, t-\tau)=G(\mathbf{y}, \mathbf{x}, t-\tau)$ applies.

When the initial and boundary conditions are the same as for the physical problem, the Green's function is called a tailored Green's function. In that case the integral form of Howe's analogy is given by:

$$
B^{\prime}=\int_{-\infty}^{\infty} \int_{V} G(\mathbf{x}, \mathbf{y}, t-\tau) \nabla_{\mathbf{y}} \cdot(\boldsymbol{\omega}(\mathbf{y}, \tau) \times \mathbf{v}(\mathbf{y}, \tau)) \mathrm{d}^{3} y \mathrm{~d} \tau
$$

Where $V$ is the volume of the system in which the source $\nabla \cdot(\boldsymbol{\omega} \times \mathbf{v})$ is nonvanishing. The integrand vanishes for all $\tau$ such that $t<\tau$ due to causality considerations. Note that it is here assumed that for $t \rightarrow-\infty B^{\prime}$ and $\partial B^{\prime} / \partial t$ are zero, i.e. the initial condition in the integral formulation of the analogy is that at the beginning of time there was no acoustic perturbation. Partial integration and assuming anechoic (non-reflecting) terminations of the channel yields

$$
B^{\prime}=-\int_{-\infty}^{\infty} \int_{V} \nabla_{\mathbf{y}} G(\mathbf{x}, \mathbf{y}, t-\tau) \cdot(\boldsymbol{\omega}(\mathbf{y}, \tau) \times \mathbf{v}(\mathbf{y}, \tau)) \mathrm{d}^{3} y \mathrm{~d} \tau .
$$

From this point the specific problem of a sudden contraction in an infinitely long channel is considered, which implies anechoic terminations. This differs from a rocket engine in that for the latter, additional surface integrals appear to take acoustic reflections into account or losses at the boundaries (chapter 2).

The gradient $\nabla_{\mathbf{y}} G(\mathbf{y}, \mathbf{x}, t-\tau)$ of the Green's function corresponds to the acoustic velocity induced at $\mathbf{y}$ by a pulse $\delta(\mathbf{x}-\mathbf{y}) \delta(t-\tau)$ released at $\mathbf{x}$. The observer is assumed to be placed upstream of the contraction in the uniform channel of section $S_{1}$.

We limit our study to low frequencies, for which the acoustic wave length is much larger than twice the channel height, so that only plane waves propagate 
over large distances. When considering acoustic waves generated by a point source located at $y_{1}=x_{1}$, the plane waves will prevail at distances of a few channel heights from the source.

The volume $V$ is chosen to enclose the point source, and is delimited by the channel walls and cross sections at a few diameters from the source. The length of the compact source region is taken to be $2 \epsilon$, such that the integration volume has boundaries with outer normals parallel to the plane wave propagation direction at $y_{1}=x_{1}-\epsilon$ (lefthand side) and $y_{1}=x_{1}+\epsilon$ (righthand side). One can integrate equation (3.31) over $V$ to find

$$
\int_{V}\left[\frac{1}{c_{0}^{2}} \frac{\partial^{2} G}{\partial t^{2}}-\nabla^{2} G\right] d^{3} y=\int_{V} \delta(\mathbf{x}-\mathbf{y}) \delta(t-\tau) \mathrm{d}^{3} y=\delta(t-\tau),
$$

because by definition $\int_{V} \delta(\mathbf{x}-\mathbf{y}) d^{3} y=1$.

When $V$ is compact (small compared to the wave length) the volume integral of the time derivative term can be neglected. Then, using the fact that $\mathbf{n} \cdot \nabla G=0$ at the walls, while the acoustic field is uniform on the other surfaces enclosing $V$ (plane wave solution) one obtains relations between the solution $\left(G^{-}\right)_{y_{1}=x_{1}-\epsilon}$ left of the source and $\left(G^{+}\right)_{y_{1}=x_{1}+\epsilon}$ right of the source:

$$
-W S_{1}\left[\left(\frac{\partial G^{+}}{\partial y_{1}}\right)_{y_{1}=x_{1}+\epsilon}-\left(\frac{\partial G^{-}}{\partial y_{1}}\right)_{y_{1}=x_{1}-\epsilon}\right]=\delta(t-\tau)
$$

where $W$ is the channel width. Symmetry requires that:

$$
\begin{aligned}
\left(G^{+}\right)_{y_{1}=x_{1}+\epsilon} & =\left(G^{-}\right)_{y_{1}=x_{1}-\epsilon} \\
\left(\frac{\partial G^{+}}{\partial y_{1}}\right)_{y_{1}=x_{1}+\epsilon} & =-\left(\frac{\partial G^{-}}{\partial y_{1}}\right)_{y_{1}=x_{1}-\epsilon}
\end{aligned}
$$

When $x>0$ one has for the $G^{-}$wave propagating towards the contraction (at $\left.x_{1}=0\right)$ a solution of d'Alembert:

$$
2 W S_{1}\left[\frac{\partial G^{-}}{\partial y_{1}}\right]=\delta\left(t-\tau+\frac{y_{1}-x_{1}}{c_{0}}\right)
$$

The pressure wave upstream of the contraction $\left(x_{1}-y_{1}\right)>0$ is obtained by integration of equation (3.38) and is given by a Heaviside time dependence

$$
G^{-}=\frac{c_{0}}{2 W S_{1}} \mathcal{H}\left(t-\tau+\frac{y_{1}-x_{1}}{c_{0}}\right)
$$


Chapter 3 - AnAlytical incompressible frictionless tWo-Dimensional

PLANAR MODEL FOR VORTEX-NOZZLE INTERACTION

At low frequency, the wave $G^{-}$induces a reflection $G_{R}^{+}$at the contraction $y=0$ given by $^{3}$

$$
\begin{aligned}
G_{R}^{+} & =G^{-} R_{B} \\
& =G^{-} \frac{S_{1}-S_{2}}{S_{1}+S_{2}} .
\end{aligned}
$$

In the contraction the Green's function is

$$
G=\frac{c_{0}}{2 W S_{1}}\left[\mathcal{H}\left(t-\tau+\frac{\phi-x_{1}}{c_{0}}\right)+\frac{S_{1}-S_{2}}{S_{1}+S_{2}} \mathcal{H}\left(t-\tau-\frac{\phi+x_{1}}{c_{0}}\right)\right]
$$

The first term between square brackets is the incoming wave and the second term is the reflected wave. Here $\phi=\phi(\mathbf{y})$ is the potential of an incompressible frictionless flow induced by a uniform flow of velocity for which $\lim _{y_{1} \rightarrow \infty} \nabla_{\mathbf{y}} \phi=$ $(1,0)$ viz. far upstream of the contraction (Howe 1975). The gradient $\nabla_{\mathbf{y}} G$ of the Green's function in the contraction is

$$
\nabla_{\mathbf{y}} G=\frac{\nabla_{\mathbf{y}} \phi}{2 W S_{1}}\left[\delta\left(t-\tau+\frac{\phi-x_{1}}{c_{0}}\right)-\frac{S_{1}-S_{2}}{S_{1}+S_{2}} \delta\left(t-\tau-\frac{\phi+x_{1}}{c_{0}}\right)\right] .
$$

As the contraction is compact, one can use $\phi<<x_{1}$, which yields

$$
\nabla_{\mathbf{y}} G \simeq \frac{S_{2} \nabla_{\mathbf{y}} \phi}{W S_{1}\left(S_{1}+S_{2}\right)} \delta\left(t-\tau-\frac{x_{1}}{c_{0}}\right)
$$

In the case of the channel considered here one has $\phi=\left(S_{1} / \pi\right) \ln \zeta$. In terms of Howe's low frequency Green's function equation (3.33) becomes:

$$
B^{\prime}=-\frac{S_{2}}{W S_{1}\left(S_{1}+S_{2}\right)} \int_{V}\left[\nabla_{\mathbf{y}} \phi \cdot(\boldsymbol{\omega}(\mathbf{y}, \tau) \times \mathbf{v}(\mathbf{y}, \tau))\right]_{\tau=t_{e}} \mathrm{~d}^{3} y
$$

where $t_{e}=t-x_{1} / c_{0}$ is the emission time. The wave propagation within the contraction is neglected (compact source). This corresponds to the result of Howe (Howe 2014) ${ }^{4}$ as used by Hulshoff et al. (Hulshoff et al. 2001). Convective effects in the wave propagation (limit $M \rightarrow 0$ ) which are taken into account by Howe (Howe 1975) and Hulshoff et al. (Hulshoff et al. 2001) are neglected here.

\footnotetext{
${ }^{3}$ At the junction due to pressure being continuous $G^{-}+G_{R}^{+}=G_{T}^{-}$and flux $S_{1}\left(G^{-}-G_{R}^{+}\right)=$ $S_{2} G_{R}^{-}$, eliminating $G_{T}^{-}$, we find $G^{-}+G_{R}^{+}=\frac{S_{1}}{S_{2}}\left(G^{-}-G_{R}^{+}\right)$. This can be used to find Eq. (3.41) in the text.

${ }^{4}$ Note that in Howe (Howe 1975; Howe 2014) $\phi$ is noted as $\phi^{*}$ which is not the complex conjugate of a potential $\phi$. The star has been introduced by Howe (Howe 1975) and is omitted here to avoid confusion.
} 
For a line vortex of circulation $\Gamma$ of position $\mathbf{y}_{\Gamma}=\left(y_{1 \Gamma}, y_{2}, 0\right)$ in a two dimensional flow $\mathbf{v}=\left(v_{1}, v_{2}, 0\right)$ the vorticity is

$$
\boldsymbol{\omega}=(0,0, \Gamma) \delta\left(y_{1}-y_{1 \Gamma}\right) \delta\left(y_{2}-y_{2 \Gamma}\right)
$$

and

$$
\nabla_{\mathbf{y}} \phi=\left(\frac{\partial \phi}{\partial y_{1}}, \frac{\partial \phi}{\partial y_{2}}, 0\right)
$$

Hence one finds:

$$
\nabla_{\mathbf{y}} \phi \cdot(\boldsymbol{\omega}(\mathbf{y}, \tau) \times \mathbf{v}(\mathbf{y}, \tau))=\Gamma \delta\left(y_{1}-y_{1 \Gamma}\right) \delta\left(y_{2}-y_{2 \Gamma}\right)\left[-v_{2} \frac{\partial \phi}{\partial y_{1}}+v_{1} \frac{\partial \phi}{\partial y_{2}}\right] .
$$

In complex potential notation this yields:

$$
B^{\prime}(\mathbf{x}, t)=-\frac{\Gamma S_{2}}{S_{1}\left(S_{1}+S_{2}\right)} \operatorname{Re}\left[i\left(\frac{d \phi}{d z}\right)_{z_{\Gamma}}^{*} \frac{\mathrm{d} z_{\Gamma}}{\mathrm{d} t}\right]_{t=t_{e}} .
$$

When considering a choked nozzle with an upstream flow Mach number $M$ one should replace the coefficient $2 S_{2} /\left(S_{1}+S_{2}\right)=1-R_{B}$ in equation (3.49) by the result of Marble and Candel (Marble and Candel 1977) .

\subsection{Results}

Consider firstly the case of a contraction $S_{2} / S_{1}$ with a sharp edge $(d=0)$. In Fig. 3.2 the path of a vortex with dimensionless vortex circulation $\tilde{\Gamma} \equiv$ $\Gamma /\left(U S_{1}\right)=0.8$ is given in a main flow $U=1$. The vortex is released at height $h / S_{1}=0.4$ far upstream of the contraction. The potential flow lines corresponding to acoustic field streamlines are shown as well. The vortex path cuts through the acoustic field streamlines just upstream of the contraction, in the region $0<x / S_{1}<0.5$. The acoustical stagnation enthalpy pulse generated is shown as function of time in Fig. 3.3. The largest amplitude of the pulse is reached around $t U / S_{1}=5.8$. As shown in Fig. 3.4 the vortex position is $x_{\Gamma} / S_{1} \simeq 0.2$ at that instant. So we clearly see the effect of the difference between vortex path and acoustic streamlines on the sound generation term $\nabla_{\mathbf{y}} \phi \cdot(\boldsymbol{\omega} \times \mathbf{v})$. Note that when the vortex path is parrallel to the vortex streamlines $\phi=$ const, the triple product in the source term vanishes. For times $t U / S_{1}<5$ Fig. 3.4 shows that $(1 / U) \mathrm{d} x_{\Gamma} / \mathrm{d} t \simeq-1$ corresponding to $U=1$.

It is interesting to note that the sound is mainly generated upstream of the contraction. This partially explains the result of Hulshoff et al. (Hulshoff et al. 
Chapter 3 - AnAlytical incompressible frictionless tWo-Dimensional PLANAR MODEL FOR VORTEX-NOZZLE INTERACTION

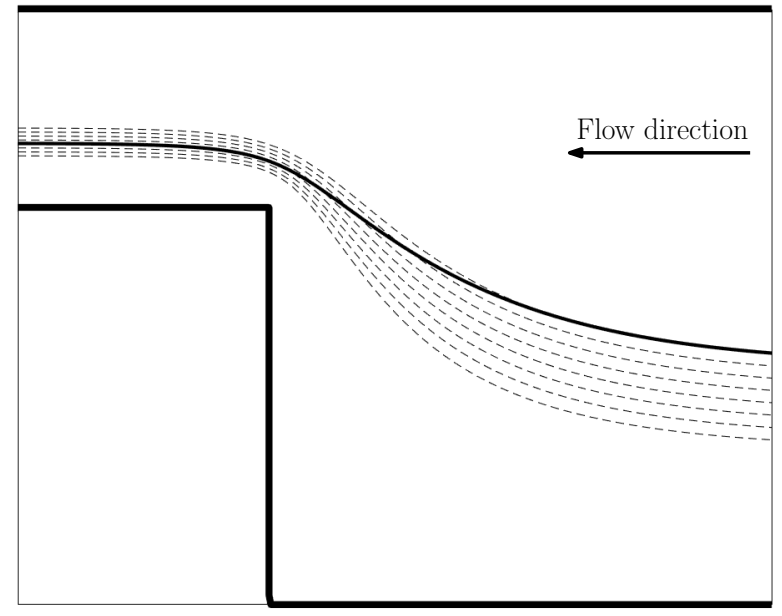

Figure 3.2: Path of vortex $|\tilde{\Gamma}|=0.8$ (solid) cutting acoustic field lines (dashed) mainly on approach to the contraction, $S_{2} / S_{1}=1 / 3, h / S_{1}=0.4$.

2001), which shows that an incompressible model yields results similar to a compressible flow model with critical nozzle (choked nozzle). As long as the upstream flow remains subsonic $M<1$ an incompressible model is a reasonable first order approximation. This justifies the use the model discussed here to obtain insight on the sound production by ingestion of vortices by a nozzle in a rocket engine.

In Fig. 3.5 the influence of variation of the height $h / S_{1}$ at which the vortex is released is considered. This corresponds to Fig. 6 of Hulshoff et al. (Hulshoff et al. 2001). The height of the pulses agrees well with the results of Hulshoff et al. (Hulshoff et al. 2001), however the time scale appears a factor three longer in the results of Hulshoff et al. (Hulshoff et al. 2001). The reason for this difference is a different scaling by Hulshoff (Hulshoff 2016c) for the data obtained with a single vortex model. Here this scaling difference has been corrected for.

Hulshoff et al. (Hulshoff et al. 2001) compare the numerical results obtained with a Euler model generating acoustic waves (at $\mathrm{M}=0.01$ ) with the results obtained by simulating the flow with a cloud of de-singularized point vortices (vortex blob method at $M=0$ ) and using the vortex sound theory to predict the sound pulse. One sees that not only the vortex sound theory correctly predicts the sound field, but also a single point vortex yields a reasonable prediction of the behavior of a cloud of vortices.

In Fig. 3.6 results obtained using the model for $d / S_{1}=0.3,|\tilde{\Gamma}|=1.5$ and 


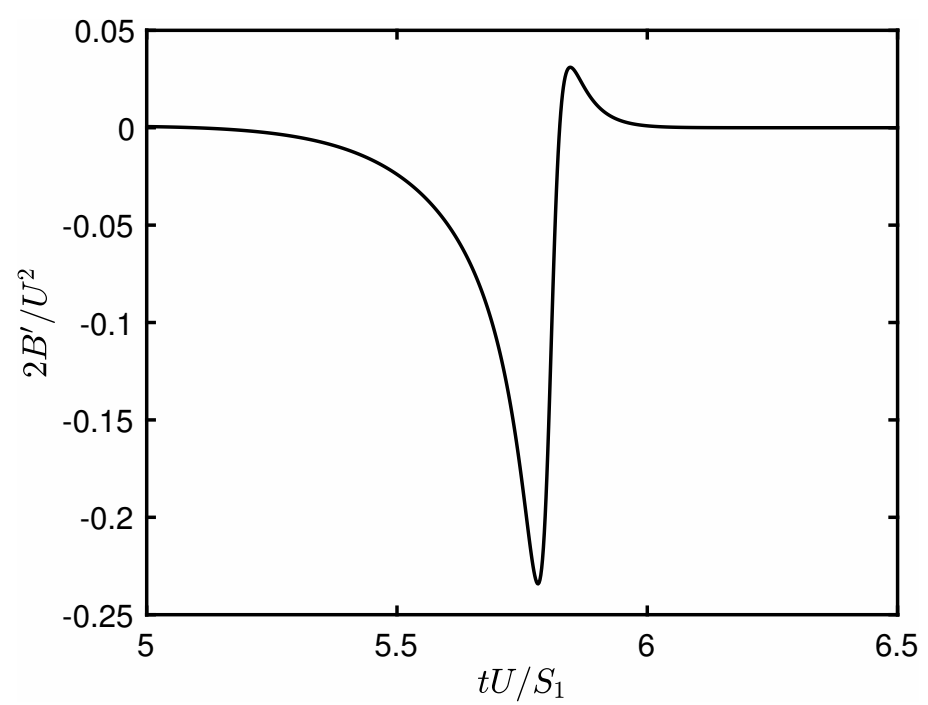

Figure 3.3: Pulse in total enthalpy $B^{\prime}$ generated by the vortex $(|\tilde{\Gamma}|=0.8)$ interaction with the contraction.

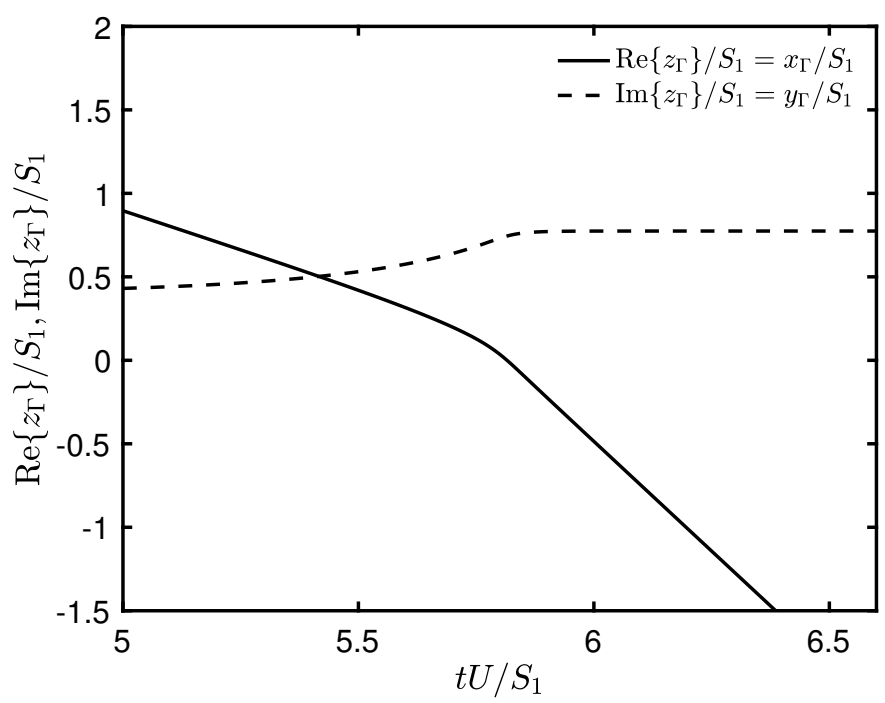

Figure 3.4: Evolution of the coordinates of the vortex $(|\tilde{\Gamma}|=0.8)$ position $\left(x_{\Gamma}, y_{\Gamma}\right)$ as a function of time. We observe that the acceleration in the horizontal velocity and the increase at $t \simeq 5.8$ in $y_{\Gamma}$ corresponds to the sound pulse production and the position $x \simeq 0.2$ around which the vortex path intersects the acoustic field lines. 
Chapter 3 - Analytical incompressible frictionless tWo-Dimensional PLANAR MODEL FOR VORTEX-NOZZLE INTERACTION

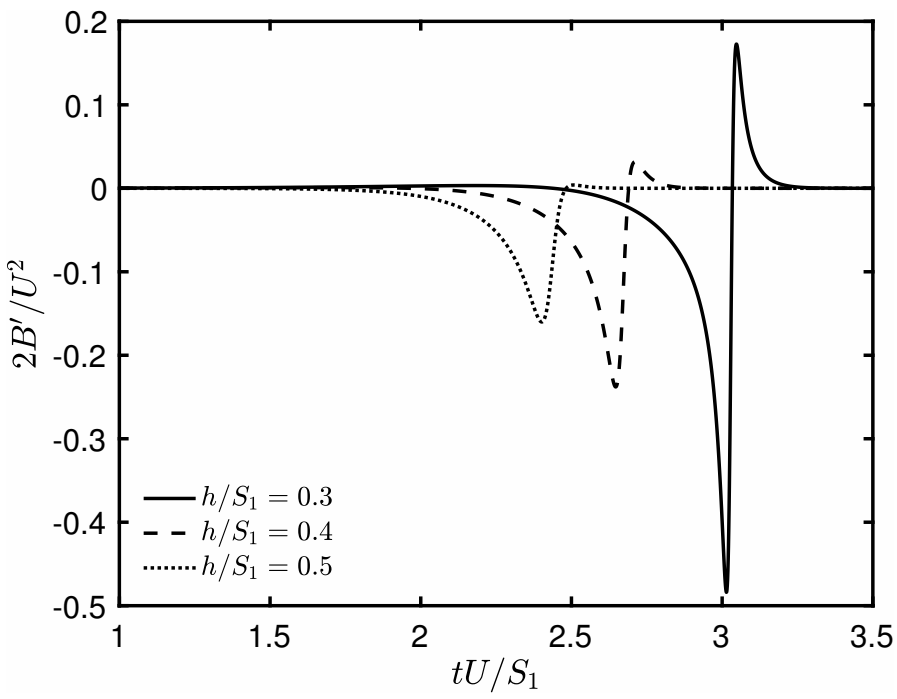

Figure 3.5: Influence of height $h / S_{1}$ at which the vortex with circulation $|\tilde{\Gamma}|=0.8$ is released on the acoustic pulse due to passage at a contraction $S_{1} / S_{2}=3$ for a sharp rectangular edge $d=0$.

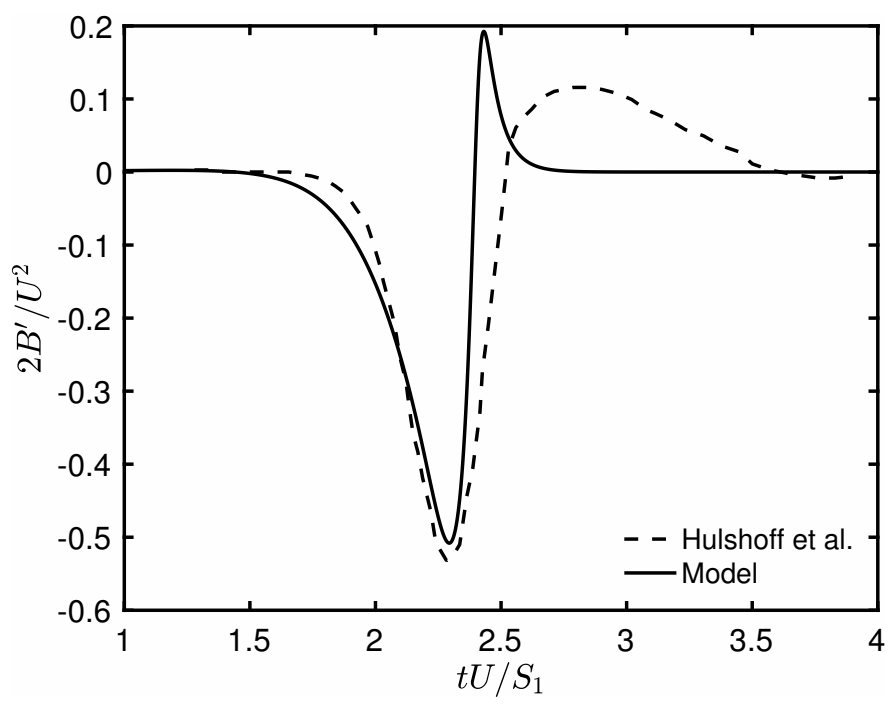

Figure 3.6: The acoustic stagnation enthalpy pulse generated for $d / S_{1}=0.3,|\tilde{\Gamma}|=$ 1.5 and $h / S_{1}=0.4$ (solid line) compared to Hulshoff et al. (Hulshoff et al. 2001) (dashed line) with $|\tilde{\Gamma}|=1.5$. 
Chapter 3 - Analytical incompressible frictionless two-dimensional PLANAR MODEL FOR VORTEX-NOZZLE INTERACTION

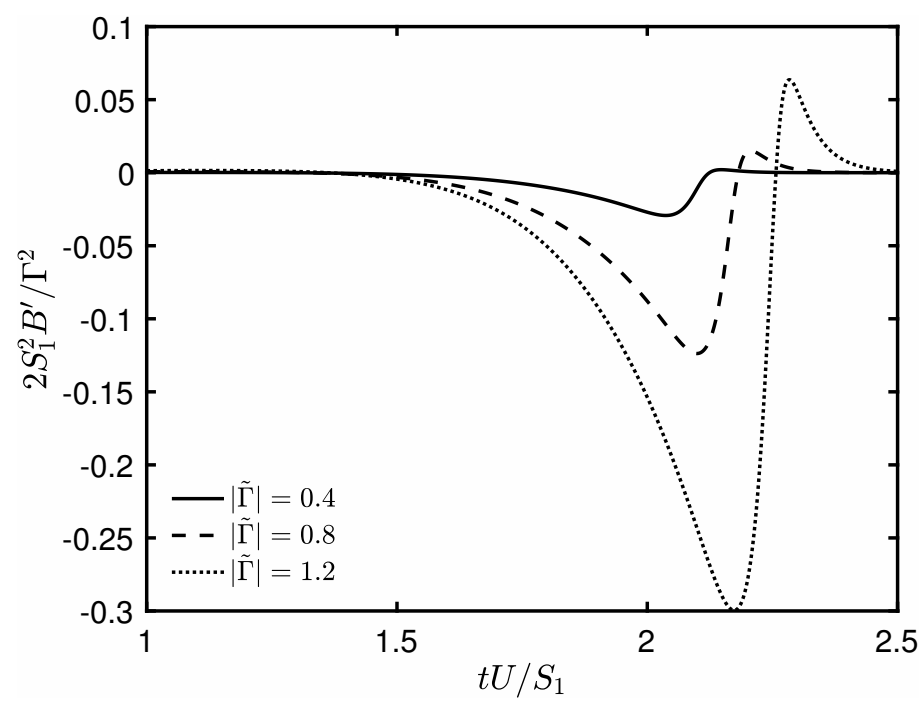

Figure 3.7: Influence of the dimensionless vortex circulation $\tilde{\Gamma}$. Vortex released at height $h / S_{1}=0.4$ on the acoustical pulse for a contraction $S_{1} / S_{2}=3$ with a nozzle inlet length $\left(d / S_{1}=1 / 3\right)$.

$h / S_{1}=0.4$ are compared to results obtained by Hulshoff et al. (Hulshoff et al. 2001) using the compressible Euler code and vortex method respectively. One observes that the large negative peak is rather well reproduced. Whilst the small positive aft peak differs. Note that our geometry only approximates the contraction part of the nozzle, Hulshoff et al. (Hulshoff et al. 2001) consider a convergent divergent nozzle while in the present work a contraction is considered. Agreement is judged satisfactory, as this positive aft peak is is not the principal contribution to vortex-nozzle interaction sound source.

The influence of the circulation is shown for vortices released at $h / S_{1}=0.4$ in Fig. 3.7 and Fig. 3.8. The effect of the circulation $\Gamma$ on the pulse amplitude is almost quadratic. This result is quite similar to the result of Hulshoff et al. (Hulshoff et al. 2001). The pulse height is again quite well predicted.

In Fig. 3.9 the influence of the shape of the edge (nozzle inlet length $d$ ) of the contraction on the acoustical pulse is shown for $0 \leq d / S_{1} \leq 1$. It is interesting to note that while the pulse depth decreases with increasing contraction length $d$, the integral of the pulse over the time appears to be almost invariant, see Fig. 3.10. For pulses short compared to the oscillation period of the combustion chamber, this implies that the oscillation amplitude is not sensitive to the shape of the nozzle. However for pulse durations comparable to or longer than the oscillation period a longer pulse involves a reduction of oscillation amplitude. 
Chapter 3 - Analytical incompressible frictionless tWo-Dimensional PLANAR MODEL FOR VORTEX-NOZZLE INTERACTION

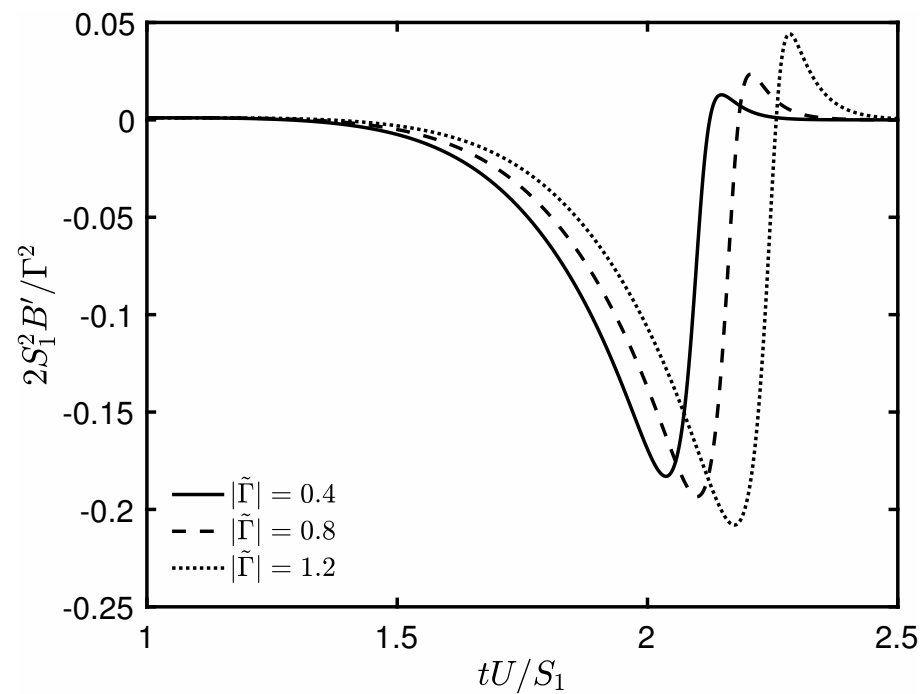

Figure 3.8: Influence of the dimensionless vortex circulation $|\tilde{\Gamma}|$ for a vortex released at height $h / S_{1}=0.4$ on the acoustical pulse for a contraction $S_{1} / S_{2}=3$ with a nozzle inlet length $\left(d / S_{1}=1 / 3\right)$. Scaled by $\frac{1}{2}\left(\frac{\Gamma}{S_{1}}\right)^{2}$. One sees that the total enthalpy perturbation scales with the square of the vortex circulation.

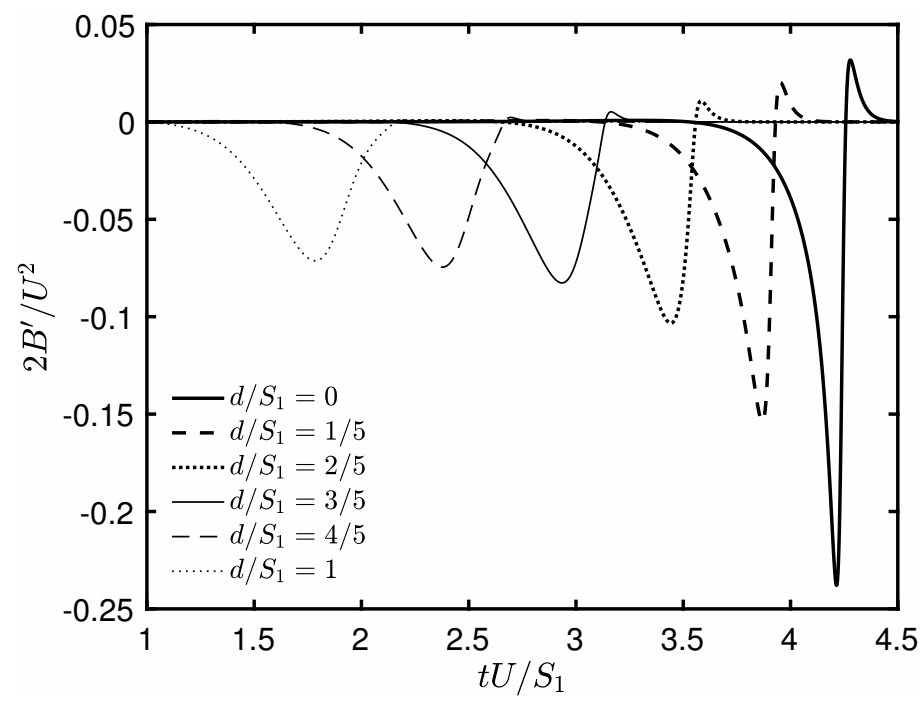

Figure 3.9: Influence of width $d / S_{1}$ of contraction (curvature of edge) sound pulse generated by the vortex with dimensionless circulation $|\tilde{\Gamma}|=0.8$ released at height $h / S_{1}=0.4$ for a contraction $S_{1} / S_{2}=3$. While the shape of the pulse is strongly affected by the geometry of the edge, the time integral of the pulse appears to remain almost constant as illustrated in Fig. 3.10. 


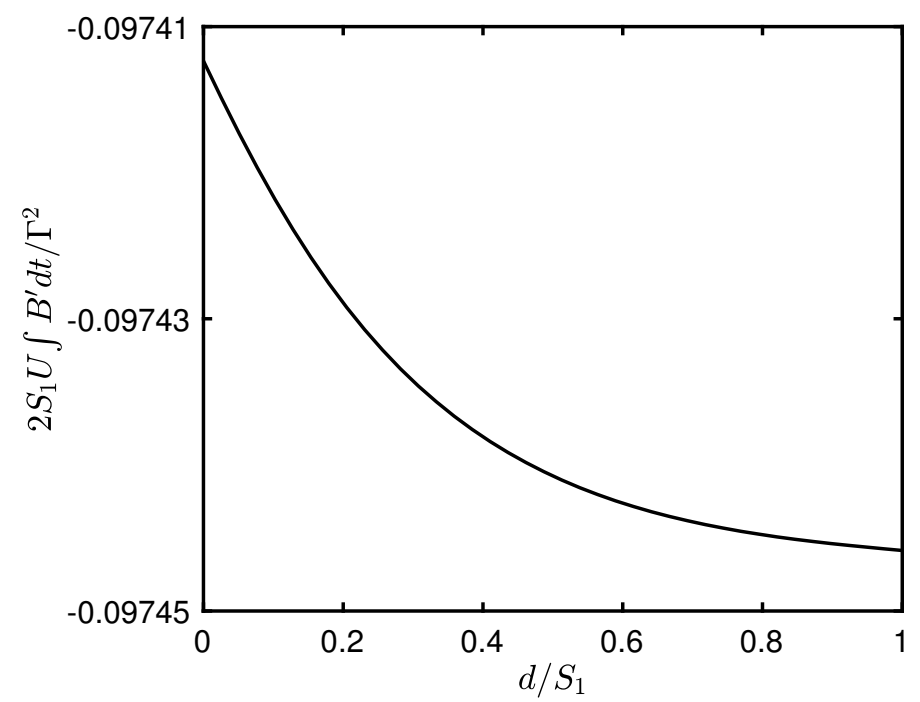

Figure 3.10: Influence of the circulation $d / S_{1}$ on the dimensionless time integral of the acoustical pulse $\frac{2 S_{1} U \int B^{\prime} \mathrm{d} t}{\Gamma^{2}}$ for a contraction $S_{1} / S_{2}=3$. The dimensionless time integral of the total enthalpy pulse is almost independent of the nozzle edge shape. It also appears to be only weakly dependent on other parameters such as the contraction ratio $S_{1} / S_{2}$, the release height $h / S_{1}$ and the dimensionless vortex circulation $\tilde{\Gamma}$.

\subsection{Conclusions}

An analytical model has been developed allowing to study the effect of nozzle parameters on the sound generated by ingestion of a vortex by a nozzle. This model considers the incompressible two-dimensional (planar) frictionless flow during passage of a line vortex through an abrupt contraction in channel height. The sound is observed to be mainly produced during the approach to the nozzle when the flow remains subsonic, so that the incompressible flow approximation is not unreasonable. The two-dimensional planar flow approximation is probably a more severe restriction. However, the model can be used for validation of a more complex numerical code or to obtain insight into the influence of parameters on the sound production.

The model has been used to study the effect of the nozzle inlet length $d$. While rounding the edge of the contraction reduces the amplitude of the stagnation enthalpy pulse, the time integral $\int B^{\prime} \mathrm{d} t$ of this pulse remains almost independent of the shape of the edge, the nozzle contraction ratio $S_{1} / S_{2}$ and the initial approach height $h$ of the vortex. This integral is approximately proportional to the square of the vortex circulation $\Gamma$. 


\title{
Chapter 4
}

\section{Vortex-nozzle interaction: two-dimensional plane Euler simulations based lumped element sound source model}

\begin{abstract}
A lumped element model for the sound source due to vortex-nozzle interaction in a Solid rocket motor (SRM) based on numerical simulations of this interaction using an Euler model for two-dimensional frictionless flow is presented. This study is limited to the acoustic response of a semi-infinite pipe terminated by a choked nozzle. The study focuses on three nozzle geometries: an integrated nozzle with a cavity surrounding the nozzle inlet, a nozzle inlet placed flush in a wall forming a right angle to the walls of the combustion chamber, and a nozzle with a gradual linear ramp inlet. The dimensionless amplitude of the acoustic pressure pulse generated by the vortex-nozzle interaction is proportional to the dynamic pressure of the upstream flow, multiplied by dimensionless vortex circulation and the upstream Mach number. The vortex-core radius and vortex approach height appear to have a minor effect. For the first two configurations, the amplitude is also proportional to an effective cavity volume, the sum of a volume taking into account the compressibility of the flow in the absence of a cavity and when present the actual cavity volume. One distinguishes the sound production due to interaction of the vortex with the effective volume and sound production due to the ingestion of the vortex by the nozzle. In the first case the sound source is represented by a fluctuating total enthalpy discontinuity, proportional to the vortex circulation, placed at the inlet of the cavity. In the second case the sound source is placed at the nozzle inlet and has an amplitude, proportional to the square of the vortex circulation. Using six fit parameters the behavior of the vortex-nozzle interaction is predicted quantitatively. In
\end{abstract}


Chapter 4 - Vortex-nozzle interaction: two-dimensional Plane Euler SIMULATIONS BASED LUMPED ELEMENT SOUND SOURCE MODEL

the Euler model with integrated nozzle, one observes minor oscillations, due to transversal acoustic oscillations of the cavity/combustion chamber system. This is not predicted by the proposed lumped element model. A gradual ramp inlet reduces the pulse amplitude, however the integral of the pressure pulse over time does not change. Hence, a reduction of limit-cycle pulsation amplitude by using a gradual ramp is only expected when the pulse duration becomes longer than the global SRMs oscillation period.

\subsection{Introduction}

Sustained acoustic oscillations at frequencies $f \sim c /(2 L)$ ( $L$ is the length of the combustion chamber and $c$ is the speed of sound) close to the first longitudinal mode of the combustion chamber can occur in solid rocket motors (SRMs). Scale model experiments demonstrate that for the integrated nozzle geometries the limit-cycle pulsation amplitude increases almost linearly with the volume $V_{c}$ of the nozzle cavity surrounding the nozzle inlet. This was verified for cold gas scale experiments, in which combustion is represented by cold air injection through porous walls (Anthoine 2000). The importance of the nozzle cavity volume was confirmed for scale-model experiments and numerical simulations involving combustion of propellant (Gallier et al. 2009).

Lumped element models are interesting as engineering tools for studying the influence of the variation of parameters on the amplitude of these spurious pulsations. In the present chapter the focus is on the development of such a lumped model, which describes the sound production due to the interaction of vortices with a choked nozzle. The formation of these vortices is assumed to be due to flow instabilities (Anthoine 2000; Casalis and Vuillot 2004; Hirschberg et al. 2018). It is also assumed that sound production occurs as a result of a hydrodynamic interaction of the fully mature vortices with the choked nozzle as these leave the combustion chamber of the SRM.

In a previous study (Hirschberg et al. 2017) a model for sound production due to vortex-nozzle interaction was proposed in which the flow is assumed to be locally two-dimensional planar frictionless incompressible and the vortices are represented as line vortices (point vortices in the plane). The prediction of sound production was obtained using vortex sound theory (Hirschberg et al. 2017). The effect of the compressibility of the gas in the cavity around the nozzle inlet was later taken into account by assuming an acoustic point volume source placed at the right angle corner between the upstream lower channel wall of the combustion chamber and the inlet of the nozzle (Hirschberg et al. 2018). Using a conformal mapping yields an analytical solution for the vortex

This chapter is in part based on a Journal of the Acoustical Society of America Express Letter. With co-authors L. Hirschberg, S. J. Hulshoff, J. Collinet, C. Schram, T. Schuller 
Chapter 4 - Vortex-nozzle interaction: two-dimensional plane Euler SIMULATIONS BASED LUMPED ELEMENT SOUND SOURCE MODEL

path, given the vortex circulation $\Gamma$ and the upstream vortex release height $h$. This model explains results of cold gas scale model experiments for vortex shedding at an orifice which simulates the presence of a thermal inhibitor (Anthoine 2000). Using a two-dimensional planar frictionless flow Euler model (EIA) Hulshoff et al. (Hulshoff et al. 2001) studied production during the ingestion of a vortex for a right angle nozzle inlet geometry without cavity around the nozzle inlet. Upon analysis of Hulshoff's et al. work it was concluded that simple scaling rules obtained from dedicated vortex-nozzle interaction simulations provide a valuable insight.

In the present chapter the Euler model (EIA) is used for a systematic numerical simulations of sound produced through vortex-nozzle interaction for three types of inlet geometries of a critical nozzle: one with a nozzle cavity, one with an inlet that forms a right angle with the upstream lower channel wall, and one with a ramp inlet. Hulshoff et al. (Hulshoff et al. 2001) only studied a single nozzle inlet geometry that formed a right angle with the lower upstream combustion chamber wall. Most of Hulshoff's et al. results were obtained with a subcritical nozzle. Results published with a critical nozzle were obtained for a single upstream Mach number only (a fixed nozzle contraction ratio of 3). In this chapter results are obtained for critical nozzles (which is closer to actual SRM flow conditions), through variation of the contraction ratio results have been obtained for upstream Mach numbers that span the range $0.05 \leq M \leq 0.2$ observed in Anthoine's experiments (Anthoine 2000). In addition a study of the influence of the following parameters has been undertaken: the dimensionless vortex circulation $\tilde{\Gamma}=\Gamma /\left(U S_{1}\right)$, the vortex-core radius $R_{\Gamma}$ and its upstream release height $h$.

From these systematic parameter studies combined with vortex sound theory, scaling laws have been derived. These scaling laws have been used to formulate a lumped element model of sound production and radiation due to vortexnozzle interaction. The lumped model provides insight into the basic physical mechanisms that play a role in the vortex-nozzle sound production. It notably explains the role played by the presence of a nozzle cavity on the acoustic response.

While vortex formation in a SRM is determined by the longitudinal acoustic oscillation of the gas in the combustion chamber, it is assumed that afterwards the hydrodynamic flow, including the trajectory of the vortex as it travels through the nozzle, is not significantly affected by this acoustic oscillation. Therefore instead of considering a closed combustion chamber, the vortex-nozzle interaction is studied in a semi-infinite channel terminated by a choked nozzle. This approach avoids long numerical calculations for the establishment of a limit-cycle. It also allows for the varying of parameters such as $R_{\Gamma}$ and $\Gamma$ independently of the physics and the special conditions that occur during limit-cycle behavior. 
Chapter 4 - Vortex-nozzle interaction: two-dimensional plane Euler SIMULATIONS BASED LUMPED ELEMENT SOUND SOURCE MODEL

In the following sections the lumped model and the numerical simulations are described in detail. In section 4.2 a description and estimation of the relevant parameter ranges is provided. The nozzle geometries used for systematic vortexnozzle interactions studies are described in section 4.3. Scaling rules for sound production due to vortex-nozzle interaction are discussed in section 4.4. The derivation of a lumped element model for sound production due to vortexnozzle interaction is provided in section 4.5. In section 4.6, a brief description of the internal Aeroacoustics code (EIA) and its use for vortex-nozzle interaction simulations is given. Results of systematic vortex-nozzle interaction simulations are described in sections 4.7, 4.8 and 4.11 obtained with the integrated cavity, the right angle inlet and gradual ramp nozzle inlet geometries respectively. The determination of a residual volume $V_{0}$ is described in section 4.9. The lumped element model is used to fit and compare to EIA data in section 4.10. A summary of conclusions is given in section 4.12.

\subsection{Relevant parameter ranges}

\subsubsection{Mach number range}

Anthoine argues that relevant Mach number range for SRMs is $0.05 \leq M \leq 0.2$ (Anthoine 2000). Thus, to numerically investigate the production of sound due to vortex nozzle interaction, five upstream Mach numbers are chosen in this range. As the nozzle is choked, changing the contraction ratio $S_{2} / S_{1}$ sets the upstream Mach number. The later can be estimated using a quasi onedimensional flow approximation (Thompson 1972), based on the ratio of inlet the cross-sectional area $A_{1}=4 S_{1}^{2}$ to the cross-sectional area of the nozzle throat $A^{*}=4 S_{1} S_{2}$. Five contraction ratios were considered $A^{*} / A_{1}=S_{1} / S_{2}=3,4,6$, 7 and 10, which correspond to upstream Mach numbers of $M=0.197,0.146$, $0.0967,0.083$ and 0.058 , respectively.

\subsubsection{Estimation of vortex-core radius $R_{\Gamma}$ and circulation $\Gamma$ from laminar computations performed by Anthoine}

In the literature there is a lack of information concerning the properties of vortices in SRMs. An exception is the report on the laminar radial injection simulations of the cold gas scale experiments of Anthoine for $M=0.09$ (Anthoine 2000). A snapshot of a resulting vorticity field is shown in Fig. 4.1. From Fig. 4.1 one can estimate the vortex-core radius $R_{\Gamma}$. The estimation of $R_{\Gamma}$ is a lower bound, as in real systems turbulence induces diffusion of vorticity, increasing $R_{\Gamma}$.

To estimate the lower bound vortex-core radius consider the vortex nearest to 
Chapter 4 - Vortex-nozzle interaction: two-dimensional Plane Euler SIMULATIONS BASED LUMPED ELEMENT SOUND SOURCE MODEL

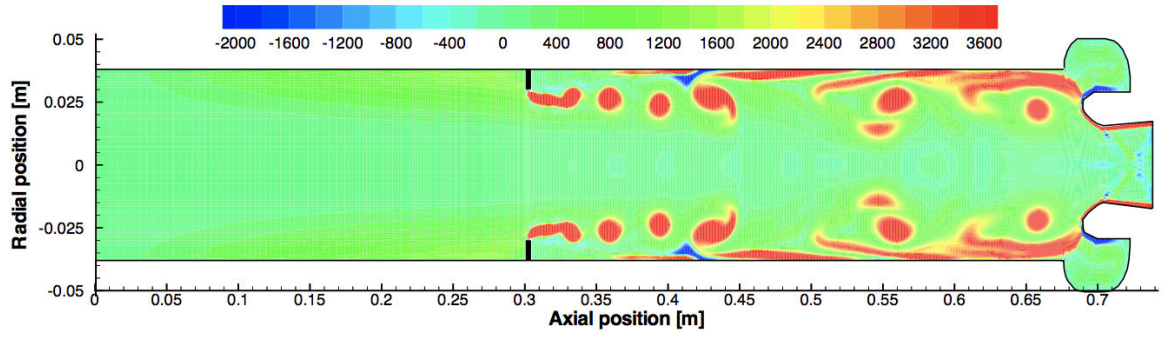

Figure 4.1: Laminar radial injection simulation taken from (Anthoine 2000). This figure is used to estimate the smallest vortex-core radius $R_{\Gamma} / S_{1}$ expected in $S R M s$ and its associated vortex circulation $\tilde{\Gamma}$. The vortex (orange red vorticity distribution) closest to the nozzle inlet is used.

the nozzle inlet viz. the circular orange red vorticity blob (Fig. 4.1). Using the scale on the left hand side provided in the figure, a dimensionless radius $R_{\Gamma} / R_{\text {pipe }}=0.13$ is found where $R_{\text {pipe }}$ is the radius of the channel upstream from the nozzle. For the Euler model (EIA) simulation of vortex nozzle interaction presented in this chapter the vortex radius was chosen to be approximately twice as large $R_{\Gamma} / S_{1}=0.3$. Furthermore, simulations were carried out with $R_{\Gamma} / S_{1}=0.2$ and 0.4 in order to study the influence of $R_{\Gamma}$ on sound production.

The vortex in Fig. 4.1 has a orange red color, which according to the color scale corresponds to $\omega_{z} \simeq 3300 \mathrm{~s}^{-1}$. The vortex circulation is estimated as follows: $\Gamma \simeq-\omega_{z} \pi R_{\Gamma}^{2}=-0.26 \mathrm{~m}^{2} / \mathrm{s}$. Which in dimensionless form corresponds to $\Gamma / U R_{\text {pipe }}=-0.22$. Where the mean velocity is estimated using $U=M c$, with $M=0.09$ the reported upstream inlet Mach number (Anthoine 2000) and the speed of sound $c=340 \mathrm{~m} / \mathrm{s}$ (for cold gas experiments).

\subsubsection{Estimation of the range of dimensionless vortex circula- tion in SRMs}

The maximum circulation $\Gamma_{\max }$ carried by vortices in SRMs, can be estimated as the product of the upstream mean flow velocity $U=M c$ at the nozzle inlet with a hydrodynamic wavelength of order $U / f$. The oscillation frequency $f$ is of the order of $c / 2 L$, where $L$ is the length of the combustion chamber.

Considering the lower half of a two-dimensional channel with upstream half channel height $S_{1}\left(A_{1}=4 S_{1}^{2}\right)$ the maximum dimensionless circulation $^{2}$ is $\left|\tilde{\Gamma}_{\max }\right|=2 L M / S_{1}$ where $\tilde{\Gamma} \equiv \Gamma /\left(S_{1} U\right)$. For typical SRMs $M=\mathcal{O}\left(10^{-1}\right)$ and $L / S_{1}=\mathcal{O}(10)$, hence $\left|\tilde{\Gamma}_{\max }\right|=\mathcal{O}(1)$. Using $\tilde{\Gamma}=\tilde{\Gamma}_{\max } / 3$, an analytical model (Hirschberg et al. 2018) was able to reproduce the order of magnitude

\footnotetext{
${ }^{2}$ Using the frequency of the first mode $f_{1}=c /(2 L)$, one estimates the hydrodynamic wavelength $\lambda_{h} \simeq U / f_{1}$, which is used to estimate the maximum circulation $\Gamma_{\max } \simeq \lambda_{h} M c=$ $2 L M^{2} c$.
} 
Chapter 4 - Vortex-nozzle interaction: two-dimensional plane Euler SIMULATIONS BASED LUMPED ELEMENT SOUND SOURCE MODEL

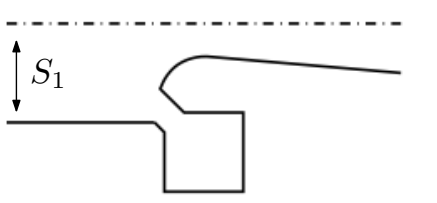

(a)

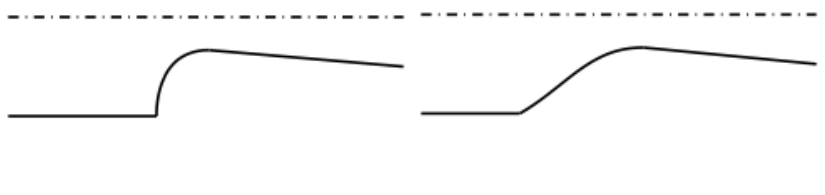

(b) (c)

Figure 4.2: $\quad$ The studied nozzle inlet geometries. Three nozzle inlet geometries considered: integrated nozzle with cavity volume (a), nozzle inlet with a right angle corner (b), and a gradual ramp nozzle inlet (c). For all inlet geometries the flow direction is from left to right. The ratio of nozzle inlet to nozzle throat cross-sectional area $A_{1} / A^{*}$ is varied to set the upstream Mach number $M$ at the inlet.

of pressure pulsation amplitudes observed in cold-gas scale experiments (Anthoine 2000). Hence, on the basis of the available information, one expects the range of circulations carried by vortices in SRMs to be $-1 \leq \tilde{\Gamma} \leq-0.1$. The negative sign of the vorticity is due to the fact that the lower half of the channel is focused on. In the upper half a positive circulation complementary vortex is present (forming a vortex pair, or two-dimensional "vortex ring")

\subsection{Nozzle inlet geometries}

For the present study three nozzle inlet geometries are considered: an integrated nozzle with cavity volume (Fig. 4.2 (a)), a nozzle inlet flush in a wall perpendicular to the side wall (forming a right angle corner Fig. 4.2 (b)), and a gradual (linear) ramp nozzle inlet (Fig. 4.2 (c)).

Sketches of a right angle inlet nozzle configuration for cylindrical and rectangular configurations respectively are shown in Fig. 4.3 (a) and (b). Fig. 4.3 is used to illustrate the relation between a rectangular channel configuration (two-dimensional plane) used in the model and the actual cylindrical configuration of e.g. scale experiments. The relation between these two configurations is through an equal contraction ratio of the upstream and throat surfaces viz. $S 1 / S 2=(R 1 / R 2)^{2}$. The upstream pipe radius $R_{1}$ and throat radius $R_{2}$ of the cylindrical tube, relates to the upstream channel height $S_{1}$ and the throat channel height $S_{2}$ of the rectangular channel, as follows $R_{1}=S_{1} \sqrt{4 / \pi}$ and $R_{2}=\sqrt{4 S_{1} S_{2} / \pi}$.

The surface used to model the presence of the integrated cavity volume $V_{c}$, was chosen of the order of a square segment $0.7 S_{1}^{2}$. This corresponds to the cavity volume $V_{c} \simeq 3 S_{1}^{3}$ or nozzle 2 used in the cold-gas scale experiments reported by Anthoine (Anthoine 2000). 
Chapter 4 - Vortex-nozzle interaction: two-dimensional plane Euler

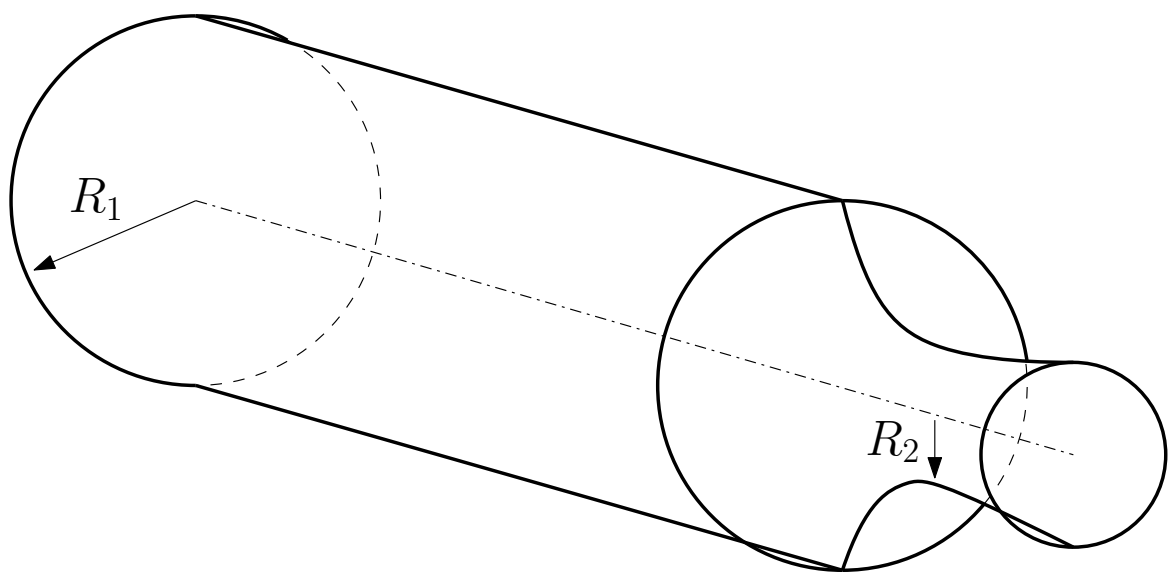

(a) Cylindrical tube with sharp angle inlet nozzle.

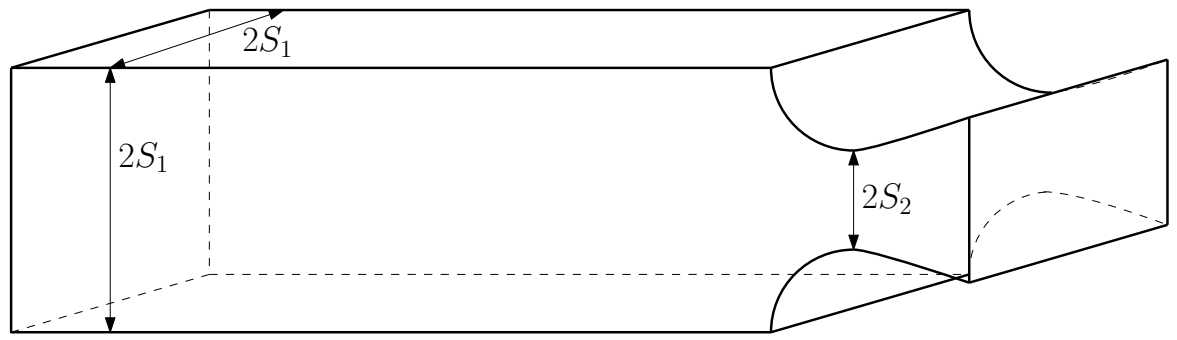

(b) Rectangular channel with sharp angle inlet nozzle.

Figure 4.3: Relation cylindrical and rectangular configuration. Where the relation between the two configurations is trough the contraction ratio $S 1 / S 2=(R 1 / R 2)^{2}$ with $R_{1}=S_{1} \sqrt{4 / \pi}$ and $R 2=\sqrt{4 S_{1} S_{2} / \pi}$. 
The right angle inlet geometry (Fig. 4.2 (b)) was drawn using Henrici's (Henrici 1974) conformal mapping as used in previous analytical models (Hirschberg et al. 2017; Hirschberg et al. 2018). This does not precisely correspond to a geometry used in scale-model experiments. However, similar right angle inlet geometries have been used in both hot-gas (with combustion) and cold-gas scale model experiments (Anthoine 2000; Gallier et al. 2009; Genot et al. 2017).

A gradual ramp inlet geometry has been used in C1xb scale experiments (Lacassagne 2017). It has been included in the present study to investigate its impact on the scaling behavior of the vortex nozzle interaction sound source and investigate the possible reduction of limit-cycle pulsation amplitude.

\subsection{Sound production and scaling rules}

\subsubsection{Low Mach number behavior without cavity}

In chapter 3 an incompressible frictionless two-dimensional plane model was considered for the interaction of a point vortex of circulation $\Gamma$ with a contraction in a channel from height $S_{1}$ to $S_{2}$. Vortex sound theory and the Green's function formalism was used to predict the upstream sound response generated in a semi-infinite channel. A fair agreement was found between this theory and the Euler model (numerical) prediction by Hulshoff et al. (Hulshoff et al. 2001) for the case of an upstream Mach number $M=0.01$ in the case of a contraction ratio $S_{1} / S_{2}=3$.

A systematic variation of parameters indicated for this incompressible model a change of the amplitude of the produced upstream acoustic wave proportional to $\tilde{\Gamma}^{2}$, where $\tilde{\Gamma} \equiv \Gamma /\left(U S_{1}\right)$ is the dimensionless vortex circulation. The theory uses a conformal mapping allowing the variation of the length $d$ of the contraction. It was found that upon variation of $d$ the time integral of the acoustic pulse $\int B^{\prime} d t$ divided by $\Gamma^{2} /\left(2 U S_{1}\right)$ remained quasi-invariant for a constant contraction ratio $S_{1} / S_{2}=3$.

The $\tilde{\Gamma}^{2}$ scaling can be understood by using the expression for the total enthalpy wave generation $B^{\prime}$ based on vortex sound theory (chapter 3 ):

$$
B^{\prime}=-\int_{-\infty}^{t} \int_{V} \nabla G \cdot(\boldsymbol{\omega} \times \mathbf{v}) \mathrm{d}^{3} y \mathrm{~d} \tau
$$

where $\boldsymbol{\omega} \equiv \nabla \times \mathbf{v}$ and $G(\mathbf{x}, \mathbf{y}, t-\tau)$ is the tailored Green's function viz. the acoustic response of the system measured at $(\mathbf{x}, t)$ for a unit pulse $\delta(t-\tau)$ released at $(\mathbf{y}, \tau)$. 
Chapter 4 - Vortex-nOzzle interaction: two-dimensional Plane Euler SIMULATIONS BASED LUMPED ELEMENT SOUND SOURCE MODEL

The Green's function $G(\mathbf{x}, \mathbf{y}, t-\tau)$ can be calculated by releasing a pulse $\delta(t-\tau)$ at the observer's position $\mathbf{x}$ and measuring the sound field at the source position $\mathbf{y}$. For large distances $|\mathbf{x}|$ and low frequencies this corresponds to the field induced by a Heaviside plane wave of amplitude $\frac{1}{2 W S_{1}} \mathcal{H}\left(t-\frac{x}{c-U}\right)$ upon reflection at the nozzle. Note that in order to obtain the correct time, the flow velocity $U$ in the channel has been inverted. This is the so-called reversed flow reciprocity principle of Howe (Howe 2014).

When considering a critical nozzle without cavity, $\nabla G$ corresponds to a potential flow through the nozzle $\mathbf{v}_{\text {pot }}$. Eq. 4.1 indicates that when convection velocity vector, $\mathbf{v}$, is parallel to the potential flow $\nabla G$, there is no sound production. Sound is produced due to deviation between the directions of $\mathbf{v}$ and $\nabla G$. There are two causes for such a deviation:

1. the influence of images in the wall of the vortex on the vortex path,

2 . the influence of local compressibility on the $\nabla G$ field at the nozzle.

The effect of images is illustrated in Fig 4.4 for a negative circulation $\Gamma=$ $\int_{S} \boldsymbol{\omega} \cdot \mathbf{n} d^{2} x$. For small values of vortex circulation magnitude $|\tilde{\Gamma}|<1$, the magnitude of the deviation between the directions of $\mathbf{v}$ and $\nabla G$ will increase linearly with $\tilde{\Gamma}$. As $\tilde{\Gamma}$ and $\boldsymbol{\omega}$ are also linearly related one expects $B^{\prime}$ to scale with $\tilde{\Gamma}^{2}$. This is indeed what one observes when using the incompressible model. The acoustic velocity $\nabla G_{r}$ related to the radiation at the nozzle will scale with $\left(1-\mathcal{R}_{B}\right)$. Using a quasi-steady approximation as proposed by Marble and Candel (Marble and Candel 1977), the plane total enthalpy wave reflection coefficient at a choked nozzle is

$$
\mathcal{R}_{B}=\left(\frac{1-\frac{\gamma-1}{2} M}{1+\frac{\gamma-1}{2} M}\right)\left(\frac{1-M}{1+M}\right)
$$

where $M=U / c$ is the Mach number of the flow at the nozzle inlet. For low Mach numbers $M^{2}<<1$ this corresponds to a proportionality factor of $(\gamma+1) M$. Hence, linear scaling of the pulse with Mach number is expected.

\subsubsection{Influence of a nozzle cavity}

When a cavity of volume $V_{c}$ is present around the nozzle inlet, the steady potential flow through the nozzle, provides the first order approximation for the convective velocity vortex, $\mathbf{v}$. The potential around the vortex path will not be strongly affected by the presence of the cavity and its volume. However, the compression of the gas in the cavity will induce an acoustic flow $\nabla G_{c}$. This is the acoustic flux induced by density variation within the cavity. 


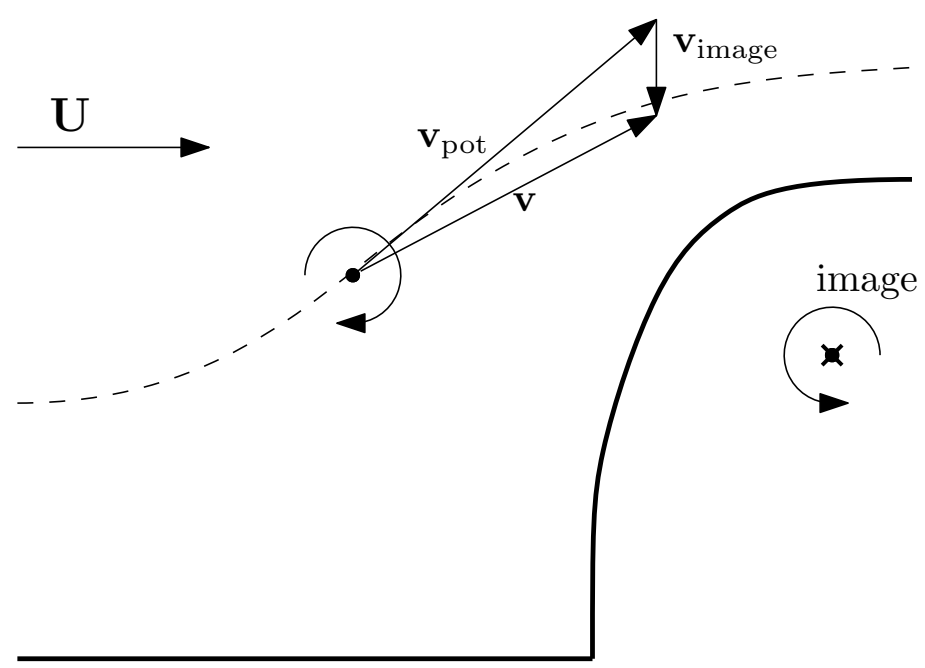

Figure 4.4: Influence of image vortex on the vortex traveling along it trajectory (dashed line) through a right angle inlet nozzle. The image induces a small deviation $\mathbf{v}_{\text {image }}$ of the vortex velocity $\mathbf{v}$ relative to the potential flow velocity $\mathbf{v}_{\mathrm{pot}} \propto \nabla G$. This deviation induced by the image is a source of sound, which scales with the square of the dimensionless vortex circulation $\tilde{\Gamma}^{2}$.

When the vortex passes along the opening of the cavity $\nabla G_{c}$ is roughly normal to the vortex convection velocity $\mathbf{v}$. This is illustrated in Fig. 4.5.

The result is a large contribution to the generation of the acoustic wave emitted upstream by vortex-nozzle interaction. Furthermore, this contribution is not expected to be sensitive to small deviations in the vortex path. $\nabla G_{c}$ is expected to scale linearly with $V_{c}$ because the cavity is small compared to the wavelength of the acoustic waves associated with self-sustained pressure pulsations, which themselves are on the order of the length of the combustion chamber. Hence, the radiated sound $B^{\prime}$ should be proportional to the product of $\tilde{\Gamma}$ and $V_{c}$.

\subsection{Lumped element model}

When considering a lumped element model for vortex sound at low frequencies in a duct such as the combustion chamber of a SRM, the acoustic field is in first order approximation described as traveling plane waves. Deviations from plane waves due to discontinuities are locally described as an incompressible potential flow. For low Mach number flows, the acoustic field is best described in terms of total enthalpy fluctuations $B^{\prime}$ (Bruggeman et al. 1991; Howe 2014). In first order approximation, the acoustic pressure fluctuation is simply $p^{\prime} \simeq \rho B^{\prime}$. Modeling of configurations such as vortex shedding at side branches or flue 


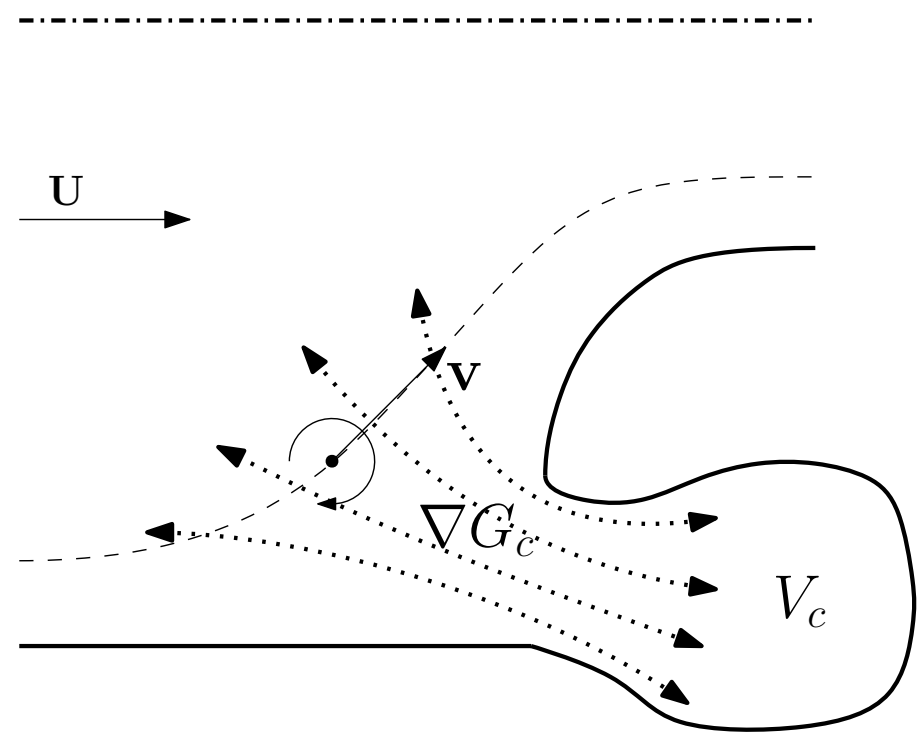

Figure 4.5: The interaction of a point vortex of circulation $\Gamma$ with the cavity compressibility induced acoustic flow $\nabla G_{c} . A s \nabla G_{c}$ is almost normal to $\mathbf{v}$, the sound production is not sensitive to deviation of the path of the vortex due to images in the wall. The sound production scales linearly with $\tilde{\Gamma}$ and $V_{c}$, and is not expected to be sensitive to variation in vortex path.

instruments demonstrates that the sound source can be represented as a total enthalpy discontinuity in the plane wave region (Verge et al. 1997; Tonon et al. 2011). The mass flux across this total enthalpy discontinuity is continuous.

As explained in section 4.4.1, in the case of vortex-nozzle interaction two sound production mechanisms have been identified. The first source is due to the deviation of the vortex path relative to acoustic field lines in the nozzle. This deviation is due to the images of the vortex as it approaches the end wall of the channel (Fig. 4.4). This occurs when the vortex is going through the nozzle inlet. This sound source will be referred to as, $\Delta B_{r}^{\prime}$, the nozzle radiation sound source.

The second sound source is due to the interaction of the vortex when it passes through the acoustic velocity field $\nabla G_{c}$ induced by the compression of the gas in the nozzle cavity (Fig. 4.5). This sound source will be referred to as, $\Delta B_{c}^{\prime}$, the compressibility sound source. The location of $\Delta B_{c}^{\prime}$ is taken to be at the nozzle cavity opening. Taking both sources into account one can formulate an acoustic lumped element model as shown in Fig. 4.6.

For small $M$, the nozzle inlet region including cavity is small compared to the characteristic wavelength $\tau c \sim S_{1} / M$ (where $\tau \sim S_{1} / U$ ). Therefore the total enthalpy can be assumed to be uniform within the cavity and at the junction 


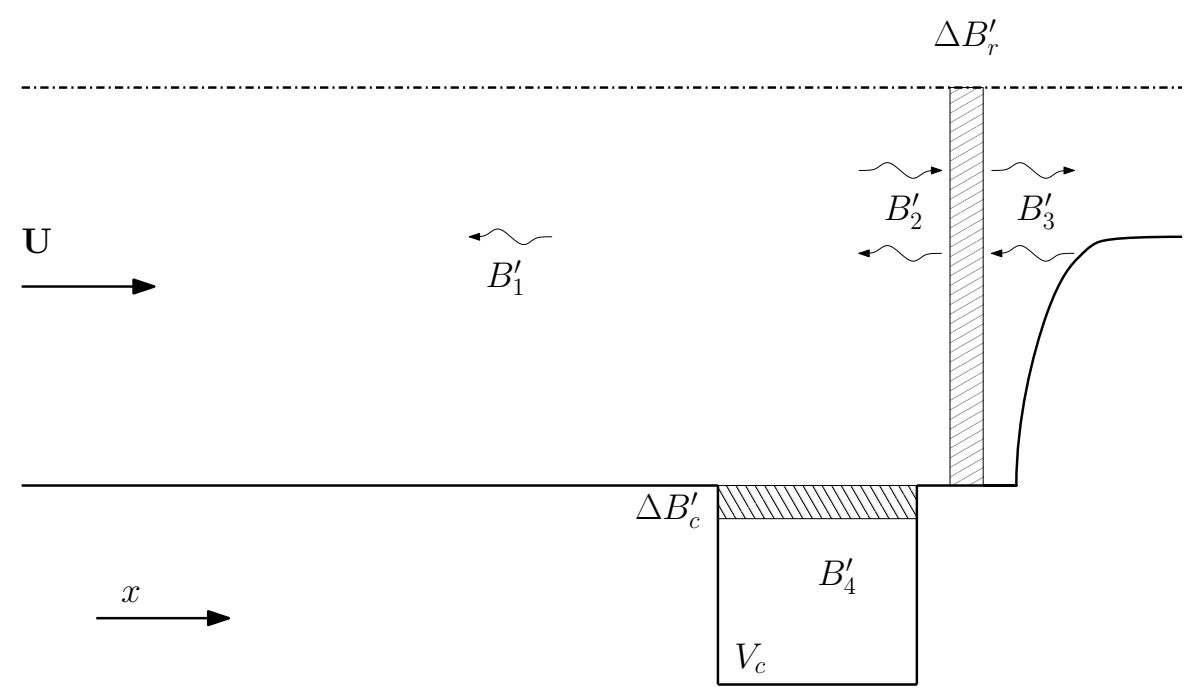

Figure 4.6: Lumped element model with two vortex sound sources $\Delta B_{c}^{\prime}$ and $\Delta B_{r}^{\prime}$.

between the cavity and nozzle inlet:

$$
B_{1}^{\prime}=B_{2}^{\prime}=B_{3}^{\prime}-\Delta B_{r}^{\prime}=B_{4}^{\prime}-\Delta B_{c}^{\prime}
$$

see Fig. 4.6 for the definition of regions 1, 2, 3, and 4. Using the continuity of mass fluxes

$$
m_{1}^{\prime}-m_{2}^{\prime}-\frac{\rho L_{\mathrm{eff}}}{c^{2}} \frac{\mathrm{d} B_{4}^{\prime}}{\mathrm{d} t}=0
$$

where $m_{i}^{\prime}=\left(\rho u_{i}\right)^{\prime}=\rho_{i} u_{i}^{\prime}+\rho_{i}^{\prime} u_{i}$ with $i=1,2,3,4$ and

$$
m_{2}^{\prime}=m_{3}^{\prime}
$$

where the mass flux fluctuation $m_{4}^{\prime}$ leaving the cavity has been calculated from the integral mass-conservation law applied to the cavity and $L_{\text {eff }}=\left(V_{c}+V_{0}\right) / S_{p}$ ( $S_{p}$ is the channel cross-sectional area). The volume $V_{\text {eff }}$ is the sum of the cavity volume $V_{c}$ and a residual volume $V_{0}$. This residual volume captures the compressibility effect of the fluid around the nozzle inlet when $V_{c}=0$ (more detail about $V_{0}$ can be found in section 4.9). Assuming plane waves in regions 1,2 and 3 :

$$
B_{i}^{\prime}=B_{i}^{+}+B_{i}^{-}
$$

one finds after some algebra:

$$
\frac{m_{i}^{\prime}}{\rho}=\frac{B_{i}^{+}}{c}-\frac{B_{i}^{-}}{c} .
$$


Chapter 4 - Vortex-nozzle interaction: two-dimensional plane Euler

Furthermore, the boundary conditions yield

$$
B_{1}^{+}=0
$$

because the channel is semi-infinite (anechoic) and

$$
B_{3}^{-}=\mathcal{R}_{B} B_{3}^{+}
$$

where the quasi-stationary model of Marble and Candel (Marble and Candel 1977) is used for $\mathcal{R}_{B}$ (Eq. 4.2).

This model leads to a differential equation for the amplitude $B_{1}^{-}$of the upstream radiated sound wave:

$$
\frac{L_{\text {eff }}}{c} \frac{\mathrm{d} B_{1}^{-}}{\mathrm{d} t}=-\frac{2 B_{1}^{-}}{1+\mathcal{R}_{B}}-\frac{1-\mathcal{R}_{B}}{1+\mathcal{R}_{B}} \Delta B_{r}^{\prime}-\frac{L_{\text {eff }}}{c} \frac{\mathrm{d} \Delta B_{c}^{\prime}}{\mathrm{d} t} .
$$

For low Mach numbers a Taylor expansion of $\mathcal{R}_{B}$ yields

$$
\mathcal{R}_{B} \simeq 1-(\gamma+1) M .
$$

Furthermore, assuming a characteristic time $\tau=S_{1} / U$ for the radiation of $\Delta B_{c}^{\prime}$ implies that $\frac{L_{\text {eff }}}{c} \frac{\mathrm{d} \Delta B_{c}^{\prime}}{\mathrm{d} t}$ also scales linearly with $M$. Hence, one expects the radiated total enthalpy wave amplitude $B_{1}^{-}$to be proportional to the upstream Mach number $M$.

Based on the discussion above, one expects that the sound source $\Delta B_{r}^{\prime}$ will scale with $\tilde{\Gamma}^{2}$ (the square of the dimensionless vortex circulation $\tilde{\Gamma}=\Gamma / U S_{1}$ ) while $\Delta B_{c}^{\prime}$ will scale linearly with $\tilde{\Gamma}$. Furthermore, both sources are supposed to be proportional to the dynamic pressure divided by the density $U^{2} / 2$. It is assumed that both sources are pulses with a time duration of the order of $S_{1} / U$.

As a first approximation, the following time-dependent sound sources are considered:

$$
\Delta B_{c}^{\prime}(t)=\left\{\begin{array}{l}
\alpha \tilde{\Gamma} \frac{U^{2}}{2} \sin ^{2}(\omega t) \text { for } 0 \leq t \leq T / 2 \\
0 \text { for all other } t
\end{array}\right.
$$

and

$$
\Delta B_{r}^{\prime}(t)=\left\{\begin{array}{l}
\beta \tilde{\Gamma}^{2} \frac{U^{2}}{2} \sin ^{2}(\omega(t-\tau)) \text { for } 0 \leq(t-\tau) \leq T / 2 \\
0 \text { for all other }(t-\tau)
\end{array}\right.
$$




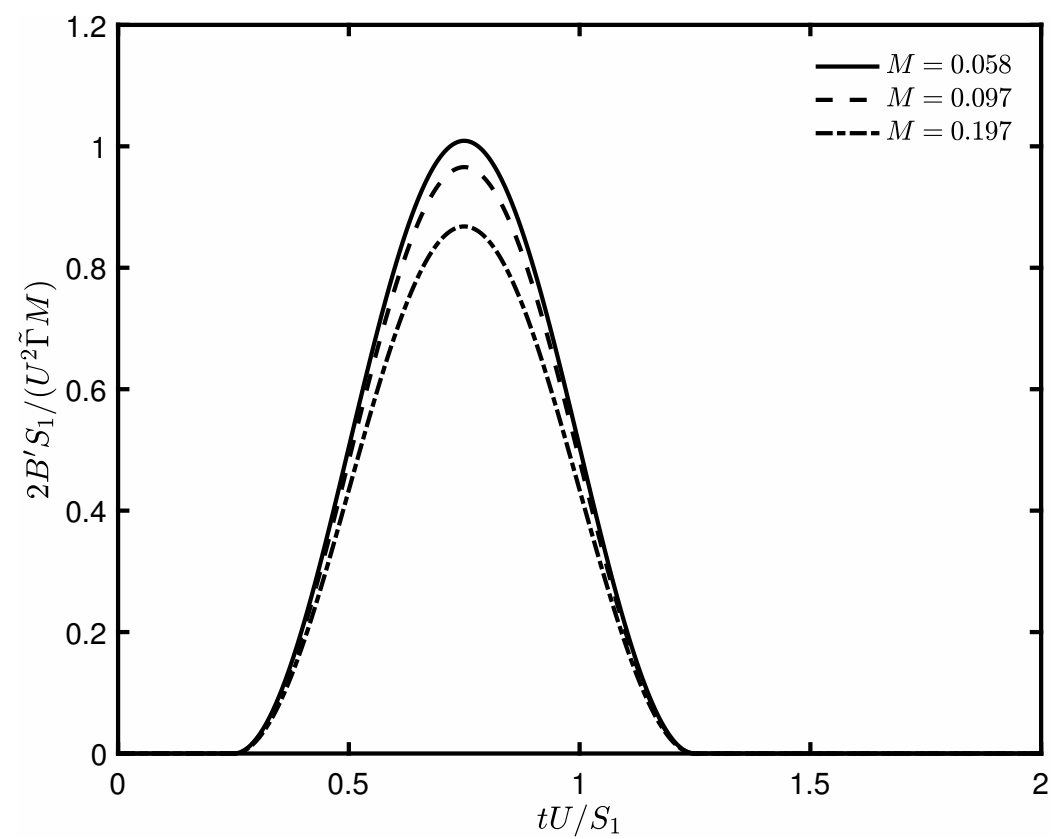

Figure 4.7: Lumped model for $L_{\mathrm{eff}}=0, \tilde{\Gamma}=-0.9, \omega=\pi U / S_{1}$ and $\alpha=\beta=1$ thus $a \sin ^{2}(\omega t)$ time-dependence of pulse $\Delta B_{r}^{\prime}$.

where the coefficients $\alpha$ and $\beta$ can for now be viewed as fit parameters. The frequency is given by $\omega=2 \pi / T=\pi U /\left(2 S_{1}\right)$ ( $T$ is the period of oscillation) and the delay between the sources is assumed to be $\tau=S_{1} /(4 U)$. It will be shown that this very basic model provides some insight into the physics of the vortexnozzle interaction sound source, when compared to numerical simulations with the Euler model (section 4.10).

In Fig. 4.7 the upstream acoustic response due to vortex-nozzle interaction, predicted using the above formulated lumped element model, is shown in absence of cavity $\left(V_{c}=0\right)$ for a vortex of dimensionless circulation $\tilde{\Gamma}=-0.9$. The total enthalpy $B^{\prime}(t)$ is normalized by dividing by $U^{2} \tilde{\Gamma} M / 2$. As $\tilde{\Gamma}<0$, the total enthalpy pulse $B^{\prime}(t)$ is negative (expansion). The normalized pulse amplitude is only weakly dependent on the nozzle Mach number $(0.05 \leq M \leq 0.2)$ and has a maximum dimensionless amplitude close to $(\gamma+1) / 2 \simeq 1.2$, corresponding to $\left(1-\mathcal{R}_{B}\right) / 2$ as expected from Eq. 4.10, when $L_{\text {eff }}=0$.

In Fig. 4.8 the results for an effective cavity length $L_{\text {eff }}=V_{c} / S_{p}=1$ with $S_{p}$ the upstream cross-sectional area of the channel. This corresponds to a value of $L_{\text {eff }}$ as expected in Ariane 5 following Anthoine (Anthoine 2000). The calculated amplitude of the acoustic response is now much larger, by a factor three compared to the case without $L_{\text {eff }}$. Its time dependence corresponds roughly to the time derivative of the original pulse $\Delta B_{c}^{\prime}$, given by Eq. (4.12). 
Chapter 4 - Vortex-nOzzle interaction: two-dimensional Plane Euler SIMULATIONS BASED LUMPED ELEMENT SOUND SOURCE MODEL

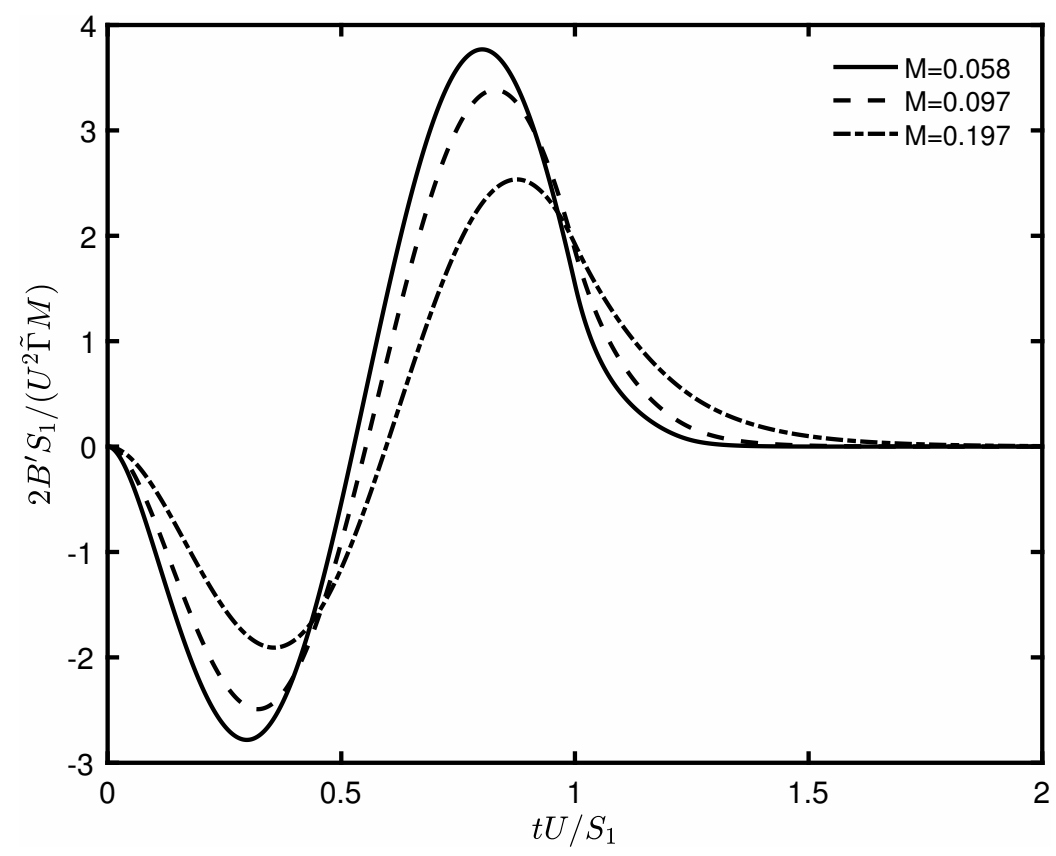

Figure 4.8: Lumped model for $L_{\mathrm{eff}}=1, \tilde{\Gamma}=-0.9, \omega=\pi U / S_{1}$ and $\alpha=\beta=1$ thus a $\sin ^{2}(\omega t)$ time dependence of pulses generated by $\Delta B_{c}^{\prime}$ and $\Delta B_{r}^{\prime}$.

This can be understood by assuming that the nozzle acts acoustically as a closed wall. In this limit the source $\Delta B_{c}^{\prime}(t)$ generates the acoustic field for an upstream observer at distance $x$ from the nozzle:

$$
B^{\prime}(x, t)=-\frac{1}{2}\left[\Delta B_{c}^{\prime}\left(t+\frac{x}{c}\right)-\Delta B_{c}^{\prime}\left(t+\frac{x-2 L_{\mathrm{eff}}}{c}\right)\right] \simeq-\left(\frac{\mathrm{d} \Delta B_{c}^{\prime}}{\mathrm{d} t}\right)_{t+\frac{x}{c}} \frac{L_{\mathrm{eff}}}{c}
$$

where the convective effects have been ignored and $L_{\mathrm{eff}} U /\left(S_{1} c\right)$ is assumed to be small. This is a dipole sound source. The first term $-\frac{1}{2} \Delta B_{c}^{\prime}\left(t+\frac{x}{c}\right)$ is the directly upstream traveling wave. The second term $\frac{1}{2} \Delta B_{c}^{\prime}\left(t+\frac{x-2 L_{\text {eff }}}{c}\right)$ is the initially downstream traveling wave reflected againt the bottom of the cavity. Experiments and numerical simulations indeed confirm that the signal with cavity approaches the shape of the time derivative predicted by Eq. (4.14).

A fit of pulses obtained using an Euler model will be performed in section 4.10. Fitting will be done by adjusting the parameters $\alpha, \beta, \tau$ and $\omega$ modifying the pulse shape. It will also be argued that $\alpha$ and $\beta$ should be proportional to some positive power of the time $t$, in order to obtain a skewed pulse shape. This should take into account the effect of the acceleration of the vortex during 
Chapter 4 - Vortex-nozzle interaction: two-dimensional plane Euler SIMULATIONS BASED LUMPED ELEMENT SOUND SOURCE MODEL

sound production (ingestion by the nozzle).

\subsection{Euler two-dimensional plane model}

In this section a description of the governing equations used for the Euler model are given (subsection 4.6.1). The meshing process is discussed in subsection 4.6.2. The establishment of a stationary base flow to initialise vortex-nozzle interaction simulations is described in subsection 4.6.3. Setting up a vortex-nozzle interaction simulation and the detection of the acoustic response produced is described in subsection 4.6.4.

\subsubsection{The governing Euler equations}

The governing equations solved by the Euler internal Aeroacoustics code (EIA) are the following

$$
\begin{aligned}
\frac{\partial \rho}{\partial t}+\nabla \cdot[\rho \mathbf{v}] & =0 \\
\frac{\partial \rho \mathbf{v}}{\partial t}+\nabla \cdot\{\rho \mathbf{v v}+p \mathbf{1}\} & =\rho \mathbf{f} \\
\frac{\partial \mathcal{E}_{t}}{\partial t}+\nabla \cdot\left[\left(\mathcal{E}_{t}+p\right) \mathbf{v}\right] & =\mathcal{Q}_{E}
\end{aligned}
$$

where $\mathcal{E}_{t}=\rho\left(e+\mathbf{v}^{2} / 2\right)$ is the total energy density (total energy per unit volume), $\rho$ f external momentum source density and $\mathcal{Q}_{E}$ the energy source. The external momentum source $\mathbf{f}$ is used to generated vortices, a description of this process is provided in subsection 4.6.4. The energy source term $\mathcal{Q}_{E}$ contains work performed by the external force density $\rho \mathbf{f}$, an optional external energy source which can be used to generate entropy spots and can be used for entropy control during vortex generation (Hulshoff 2018). Entropy control was not used as early comparisons of results with and without this function showed a marginal influence on the results. The ideal gas law was used as an equation of state.

\subsubsection{Mesh generation}

Three types of meshes have been generated: a right angle nozzle inlet (Fig. 4.2 (a)), an integrated nozzle with a nozzle cavity (Fig. 4.2 (b)) and a gradual ramp inlet (Fig. 4.2 (c)). In this subsection details of mesh generation for the right angle inlet nozzle configuration are given. The description broadly applies to the integrated nozzle and ramp inlet nozzle configurations as well.

In Fig. 4.9 the mesh for the nozzle configuration with a right angle inlet is shown. The mesh consists of from left to right Blocks 1 (green), 2 (red), 3 (yellow) and 4 (blue). Vortices are generated in Block 1, in this block the cells have a square shape. The vortices are generated by application of a non-uniform external force density $\rho \mathbf{f}$ (details in subsection 4.6.4) in this block. Block 2 serves 
Chapter 4 - Vortex-nozzle interaction: two-dimensional Plane Euler SIMULATIONS BASED LUMPED ELEMENT SOUND SOURCE MODEL

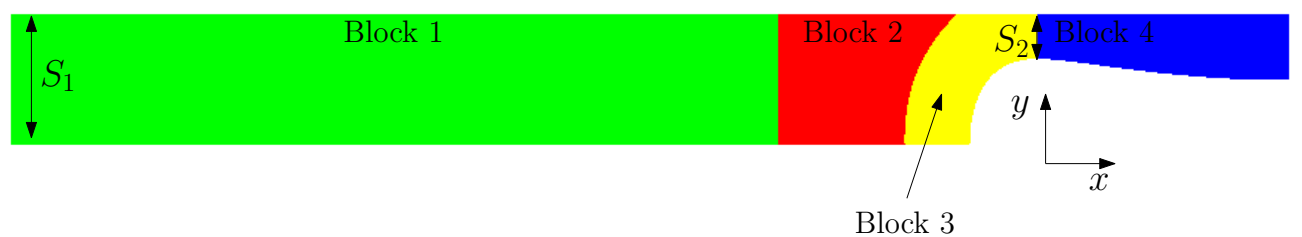

Figure 4.9: Example of right angle inlet nozzle mesh.

as a transition from the generation block to Block 3. This is done to gradually change the mesh geometry whilst keeping the cells as square as possible. Block 3 contains the contraction (nozzle). The lower wall of the contraction in Block 3 is generated using the Henrici transformation (Henrici 1974) for the analytical model (with contraction length $d / S_{1}=1 / 2$ chapter 3 ). Block 4 is the diverging channel downstream from the contraction. The height of the upstream channel is $S_{1}$ and that of the throat is $S_{2}$. The contraction ratio $S_{2} / S_{1}$ of the mesh in Fig. 4.9 is 3, which corresponds, for a critical nozzle, to an upstream Mach number of $M=0.197$. This contraction ratio is varied to change the upstream Mach number $M$. The discussion is restricted to choked nozzles.

The number of points the mesh consists of is largely determined by the need to have sufficient number of points per core-radius $R_{\Gamma}$ of the generated vortex to ensure grid independent solutions. The spatial discretization used for the vortex-nozzle interaction simulations was based on a second-order totalvariation-diminishing (TVD) Roe approximate Riemann solver with a van Leer limiter (Venkatakrishnan and Jameson 1988). A five stage Runge-Kutta time marching method was used for time integration. Using three meshes with 36, 54 and 81 points per vortex-core radius $R_{\Gamma}=0.3 S_{1}$ the estimated order of accuracy (Hulshoff 2016a) was determined to be 1.8 with a discretization error of approximately 1\% (appendix F) for the current mesh.

Grid studies confirm that using a mesh with 120 points on the upstream inlet wall, (resulting in approximately points (36) per vortex-core radius $R_{\Gamma}=0.3 S_{1}$ ) is sufficient to be in the asymptote region. The same number of points on the lefthand (inlet) boundary of Block 1 have been used on the righthand side of Block 1 as well as the left- and righthand sides of Block 2. Furthermore, the number of points on the upper and lower channel walls of Block 1 are chosen such that the resulting cells are to a satisfactory degree square. The change of cells surface from cell to cell was kept as small as possible. In addition, the number of points on the sides of the individual blocks was kept divisible by four to allow a three level explicit multi-grid (EMG) method to be used to initialize the Euler base flow (Hulshoff 2016b; Hulshoff 2018). The initialization 
Chapter 4 - Vortex-nozzle interaction: two-dimensional plane Euler SIMULATIONS BASED LUMPED ELEMENT SOUND SOURCE MODEL

procedure of the Euler base flow is briefly described in the following section.

\subsubsection{Initialization of the Euler model base flow without pres- ence of vortex}

Before the vortex nozzle interaction can be studied on Euler solution without vortices in the domain has to be set as an initial condition i.e. a stationary base flow has to be calculated. The boundary conditions (Hulshoff 2016b) imposed to find the base flow are as listed below.

- A "Usoft" boundary condition on the upstream wall (on the lefthand side of Fig. 4.9) this boundary condition imposes:

- a desired normal time averaged inflow velocity $U_{\text {des }}$,

- the local speed of sound (which is set to one),

- the local density (which is set to one).

- Wall boundary conditions on the lower and upper walls of the channel (vanishing normal velocity).

- Connection boundary conditions on all the interfaces connecting the constituent blocks of the channel.

- A non-reflective boundary condition on the downstream domain boundary.

To determine the upstream inlet velocity, $U_{\text {des }}$, for a choked nozzle, the quasione dimensional isentropic approximation:

$$
S_{2} / S_{1}=M\left(\frac{\gamma+1}{2\left(1+\frac{\gamma-1}{2} M^{2}\right)}\right)^{\frac{\gamma+1}{2(\gamma-1)}}
$$

is used (Thompson 1972). The equation is solved numerically to determine which inlet Mach number $M$ corresponds to the critical Mach number for choking.

The individual blocks of the mesh are given initial values of the density, $\rho_{\text {initial }}$, the velocity $\mathbf{v}_{\text {initial }}=(u, v)$ and the pressure $p_{\text {initial }}$. For the case of the right angle nozzle inlet (Fig. 4.9) in Blocks 1, 2 and 3 the following values are set, $\rho_{\text {initial }}=1, \mathbf{v}_{\text {initial }}=\left(U_{\text {des }}, 0\right)$ and $p_{\text {initial }}=c^{2} \rho_{\text {initial }} / \gamma$ where $\gamma$ is the Poisson constant for diatomic gas and $c$ is the speed of sound which is fixed to one in the upstream blocks 1 and 2 through the "usoft" boundary condition (Hulshoff 2016b). In the downstream section of the mesh (Block 4), the state values are set to $\rho_{\text {initial }}=1, \mathbf{v}_{\text {initial }}=(1,0)$ and $p_{\text {initial }}=c^{2} \rho_{\text {initial }} /(2 \gamma)$. Consequently, the pressure in Block 4 is lower by a factor two compared to the upstream Blocks 1,2 , and 3 . This ensures that no shock wave occurs downstream from the sonic line in the contraction and that the flow that will ultimately be reached remains supersonic in Block 4. 
Chapter 4 - Vortex-nozzle interaction: two-dimensional plane Euler SIMULATIONS BASED LUMPED ELEMENT SOUND SOURCE MODEL

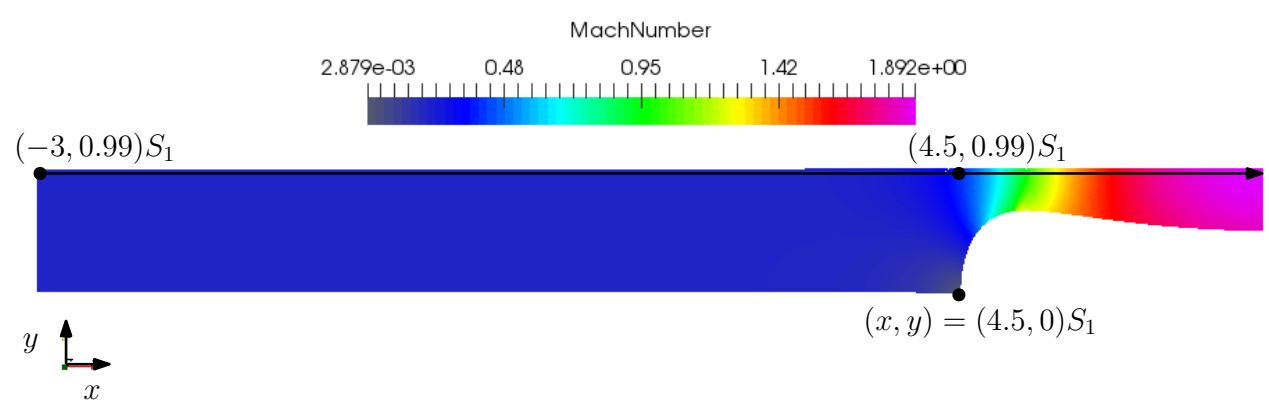

(a) Initial condition base flow for critical nozzle contraction ratio $S_{1} / S_{2}=3$ i.e. an upstream inlet Mach number $M=0.197$

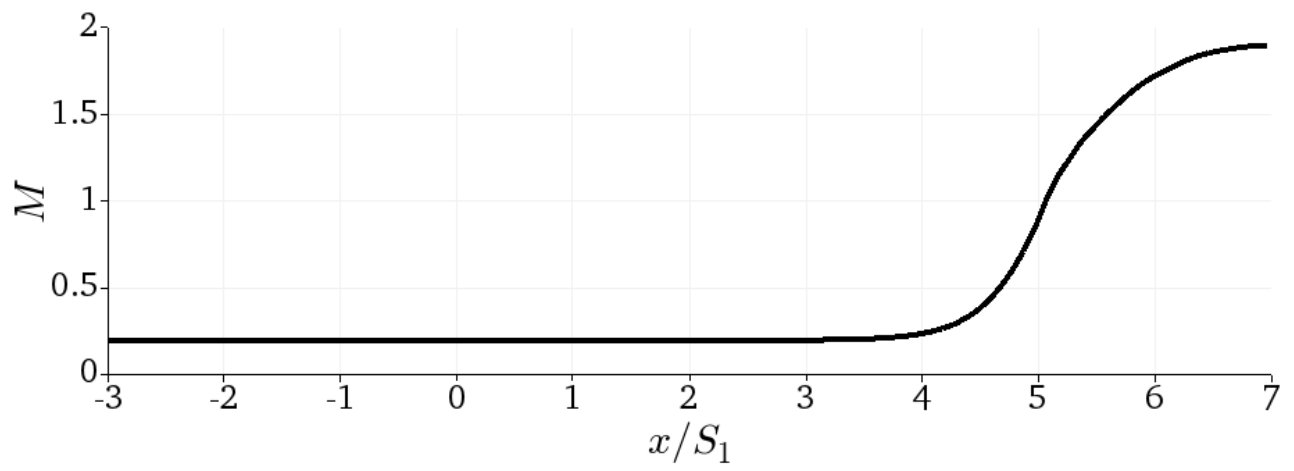

(b) Value of the local Mach number along the arrow at $y=0.99$ viz. close to the symmetry line in the $x-y$ plane shown in (a).

Figure 4.10: Initial condition base flow for $M=0.05$. 
The initial condition base flow (IC) to be used for vortex-nozzle interaction simulations is calculated using a three level explicit multi grid relaxation scheme. Spatial integration is performed using a second order total variation diminishing (TVD) Roe approximate Riemann solver with a van Leer limiter (Venkatakrishnan and Jameson 1988; Hulshoff 2016b). Time marching is done with a $(5,2)$ Runge-Kutta scheme with a Courant number $C o=3$ (Hulshoff 2016b). The calculation is run for a hundred-thousand $\left(10^{5}\right)$ time steps. A resulting IC for a contraction ratio $S_{1} / S_{2}=3$ or an upstream inlet Mach number $M=0.197$ is shown in Fig. 4.10.

\subsubsection{Vortex generation and boundary conditions used for vortex- nozzle interaction}

To study vortex-nozzle interaction a vortex needs to be generated. This is done starting from the vortex-less base flow, the established flow was described in subsection 4.6.3.

The boundary conditions used in vortex-nozzle interaction simulations are as follows.

- An acoustically non-reflective condition on the upstream wall (on the lefthand side of Fig. 4.9). This boundary condition:

- mimics a connection to an infinite upstream channel,

- maintains the local average inflow velocity, density and sound speed imposed by the base flow.

- Wall boundary conditions on the lower and upper walls of the channel.

- Connection boundary conditions on all the interfaces connecting the constituent blocks of the channel.

- A non-reflective boundary condition on the downstream end boundary.

A few snapshots of a vortex during the generation process are shown in Fig. 4.11. The snapshots were obtained with upstream Mach number $M=0.197$, release height $h=0.4 S_{1}$, vortex-core radius $R_{\Gamma}=0.3 S_{1}$. The point at which the generation process of this vortex was started was $\mathbf{x}_{0}=\left(x_{0}, y_{0}\right)=(-2,0.4) S_{1}$.

To generate a fully mature vortex like the one shown in Fig. 4.11 (c), a momentum source $\mathbf{f}$ is used. This source causes the generation of a vortex by means of the local injection of momentum around a point which is advected with the base flow $^{3}$. The momentum source $\mathbf{f}=\left(f_{x}, f_{y}\right)$ used is

$$
\begin{aligned}
& f_{x}=\mathcal{A}_{\text {local }} \sin (\theta) \\
& f_{y}=-\mathcal{A}_{\text {local }} \cos (\theta)
\end{aligned}
$$

\footnotetext{
${ }^{3}$ At low Mach numbers $M^{2}<1$ the two dimensional vorticity transport equation is $\frac{\partial \omega_{z}}{\partial t}+$ $\mathbf{v} \cdot \nabla \omega_{z}=\frac{\partial f_{y}}{\partial x}+\frac{\partial f_{x}}{\partial y}$ i.e. to generate a vortex the momentum source $\mathbf{f}$ has to be convected with the flow (Saffman 1992).
} 
Chapter 4 - Vortex-nozzle interaction: two-dimensional plane Euler SIMULATIONS BASED LUMPED ELEMENT SOUND SOURCE MODEL

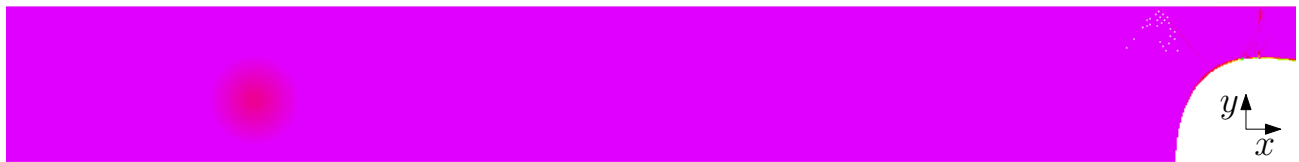

(a) $t U / S_{1} \simeq 0.59$

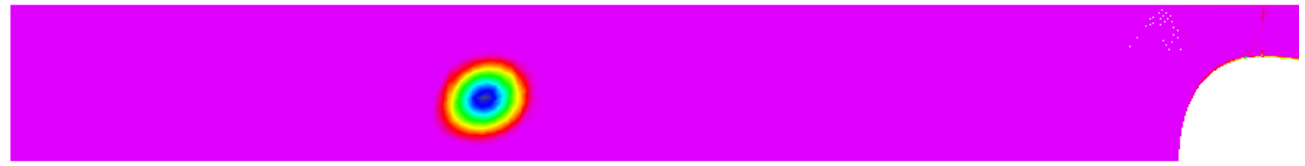

(b) $t U / S_{1} \simeq 2.1$

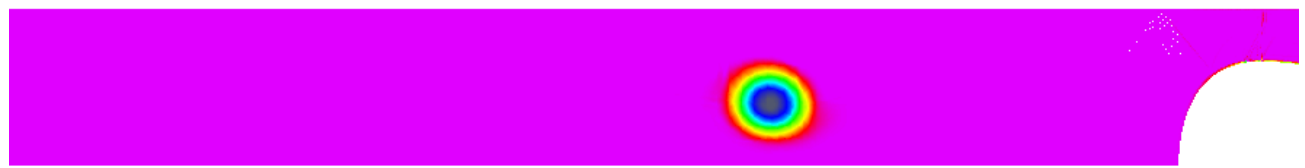

(c) $t U / S_{1} \simeq 4.0$

Figure 4.11: Snapshots of vortex during the generation process. The results shown here were obtained with upstream Mach number $M=0.197$, release height $h=0.4 S_{1}$, vortex-core radius $R_{\Gamma}=0.3 S_{1}$. The generation time interval is $0 \leq t / U / S_{1} \leq 3.0$. In subfigures $(a)$ and $(b)$ the vortex is still being generated, whilst in subfigure $(c)$ a fully mature vortex with vortex circulation $\tilde{\Gamma}=-0.86$ is shown.

where the angle $\theta$ is defined in Fig. 4.12. The position of a point $\mathbf{x}_{\text {relative }}$ vis-á-vis the moving source center is described in polar coordinates $\mathbf{x}_{\text {relative }}=$ $r(\cos (\theta), \sin (\theta))$ with $r=\left|\mathbf{x}_{\text {relative }}\right|$. The amount of momentum injected varies with respect to the distance $r$ from the source center which moves with the flow, where by definition $r>0$. This variation is controlled by a local $\mathcal{A}_{\text {local }}$ amplitude which depends on the distance $r$ is as follows

$$
\mathcal{A}_{\text {local }}=\frac{\mathcal{A}_{\text {core }}}{2 \pi r}
$$

where the core amplitude $\mathcal{A}_{\text {core }}$ is

$$
\mathcal{A}_{\text {core }}=\left\{\begin{array}{l}
\mathcal{A}\left[-2\left(\frac{r}{R_{\Gamma}}\right)^{3}+3\left(\frac{r}{R_{\Gamma}}\right)^{2}\right] \text { if } \frac{r}{R_{\Gamma}} \in[0,1) \\
\mathcal{A} \forall \frac{r}{R_{\Gamma}} \notin[0,1)
\end{array} .\right.
$$

One observes the local momentum source is irrotational $(\nabla \times \mathbf{f}=0)$ for $r>R_{\Gamma}$. Vorticity is only produced for $r<R_{\Gamma}$. The vortex-core radius $R_{\Gamma}$ is a user chosen quantity. The local amplitude $\mathcal{A}_{\text {local }}$ depends on a global amplitude $\mathcal{A}$. 
Chapter 4 - Vortex-nozzle interaction: two-dimensional plane Euler SIMULATIONS BASED LUMPED ELEMENT SOUND SOURCE MODEL

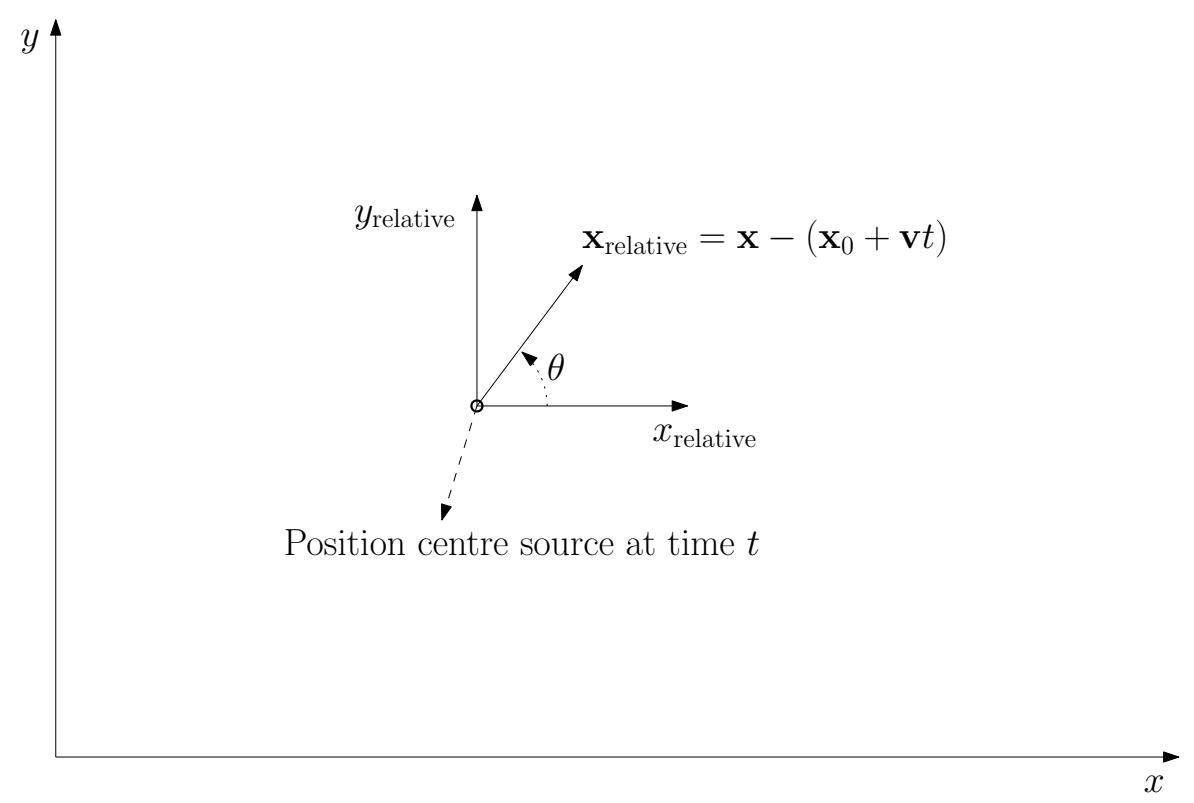

Figure 4.12: Definition of relative coordinates for momentum source $\mathbf{f}$. The position of a point with respect to the moving source center is described with local polar coordinates viz. $\mathbf{x}_{\text {relative }}=r(\cos (\theta), \sin (\theta))$ with $r=\left|\mathbf{x}_{\text {relative }}\right|$.

This global amplitude is determined as follows

$\mathcal{A}=\left\{\begin{array}{l}0.5 \mathcal{A}_{\max }\left[1-\cos \left(\frac{\pi t}{\tau_{\text {start }}}\right)\right] \text { if } t \in\left[0, \tau_{\text {start }}\right] \\ \mathcal{A}_{\text {max }} \text { if } t \in\left(\tau_{\text {start }}, \tau_{\text {start }}+\tau_{\text {max }}\right] \\ 0.5 \mathcal{A}_{\max }\left[1+\cos \left(\frac{\pi\left(t-\left(\tau_{\text {start }}+\tau_{\text {max }}\right)\right)}{\tau_{\text {end }}}\right)\right] \text { if } t \in\left(\tau_{\text {start }}+\tau_{\text {max }}, \tau_{\text {start }}+\tau_{\text {max }}+\tau_{\text {end }}\right] \\ 0 \text { for } t \in\left(\tau_{\text {start }}+\tau_{\text {max }}+\tau_{\text {end }}, t_{\text {end }}\right)\end{array}\right.$

where the maximum global amplitude $\mathcal{A}_{\max }$, the startup time $\tau_{\text {start }}$ of generation, the amount of time $\tau_{\max }$ at which the generation is done with the maximum global amplitude, the time $\tau_{\text {end }}$ during which the generation process is ramped down are chosen by the user, $t=t_{\text {end }}$ is the time at which the simulation ends. I.e. vortex generation comprises three phases of duration $\tau_{\text {start }}$, $\tau_{\max }$ and $\tau_{\text {end }}$.

In Fig. 4.13 an example of the evolution of the dimensionless vortex circulation form $\tilde{\Gamma}=0$ to -0.86 during the generation process is shown. The results shown in Fig. 4.13 were obtained with maximum amplitude $\mathcal{A}_{\max }=1.92 \times 10^{-2}$, $R_{\Gamma}=0.3, \tau_{\text {start }} U / S_{1}=1.18, \tau_{\max } U / S_{1}=0.592$ and,$\tau_{\text {end }} U / S_{1}=1.18$. The initial position of the vortex was $\mathbf{x}_{0}=(-2,0.4) S_{1}$ i.e. an upstream vortex release height $h=0.4 S_{1}$. In Fig. 4.14 the vorticity distribution for this case is 
Chapter 4 - Vortex-nOzzle interaction: two-dimensional Plane Euler SIMULATIONS BASED LUMPED ELEMENT SOUND SOURCE MODEL

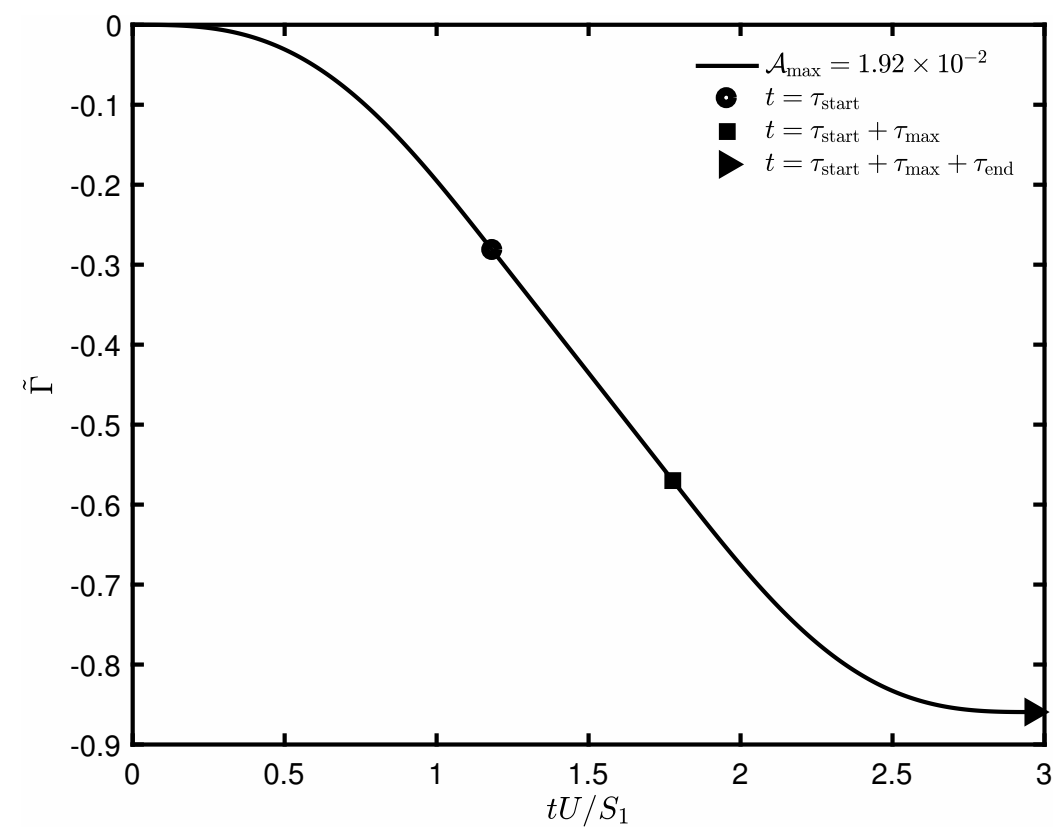

Figure 4.13: Generation phase of vortex circulation $\tilde{\Gamma}=-0.86$. To generate this vortex the user choses the amount of time during which of the vortex is being generated with the maximum global amplitude $\mathcal{A}_{\max }$ which in this case is $\tau_{\max } U / S_{1}=0.592$ and $\mathcal{A}_{\text {max }}=1.92 \times 10^{-2}$ respectively. The amount of time it takes to ramp up the global vortex generation amplitude $\mathcal{A}$ to this user set maximum is user difined and in this case equal to $\tau_{\text {start }} U / S_{1}=1.18$. To ramp it down the generation process the global amplitude $\mathcal{A}$ is lowered to zero in a time interval $\tau_{\text {end }} U / S_{1}$, which in this case is 1.18 . The end of intervals $\tau_{\text {start }}, \tau_{\max }$ and $\tau_{\text {end }}$ are indicated with a circle, a square and side-ways triangle respectively.

shown at four different times: during the startup phase, during the generation with $\mathcal{A}_{\max }$, the end of vortex generation and full maturity.

When the generation process is completed, the vortex circulation is conserved. The mature vortex is convected downstream with the base flow where eventually it passes thorough the nozzle as shown in Fig. 4.15 (a), (b),...(g). As the vortex approaches and passes through the nozzle, sound is produced. This process is referred to as vortex-nozzle interaction. In the following sections (4.7, 4.8 and 4.11) systematic parameter studies of this vortex-nozzle interaction are reported, for the three different nozzle inlet geometries (Fig. 4.2).

To record the upstream acoustic response, $p^{\prime}$, a probe is placed at $\mathbf{x}_{\text {probe }}=$ $(-2.75,0.5) S_{1}$ close to the upstream end wall (Fig. 4.16). This probe records the pressure $p_{\text {probe }}=p_{\text {probe }}(t)$ at this point. The acoustic response is determined using $p^{\prime}=p_{\text {probe }}(t)-p_{\text {probe }}\left(t_{\text {end }}\right)$. The simulations are run for a time sufficiently long so that at the end, when $t=t_{\text {end }}$, the field has returned to its 
Chapter 4 - Vortex-nozzle interaction: two-dimensional plane Euler SIMULATIONS BASED LUMPED ELEMENT SOUND SOURCE MODEL

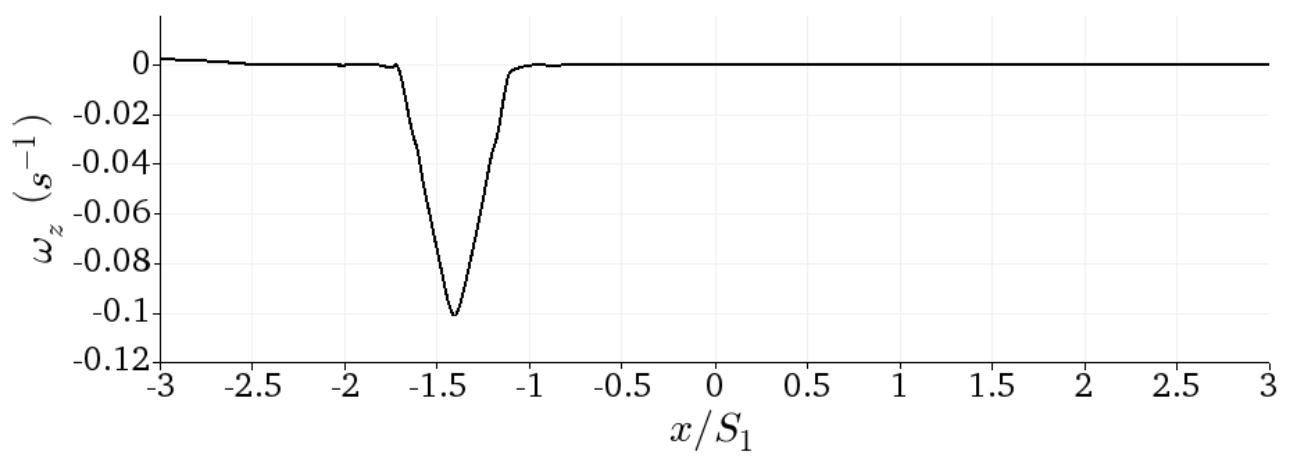

(a) $t U / S_{1} \simeq 0.59$ during the startup phase

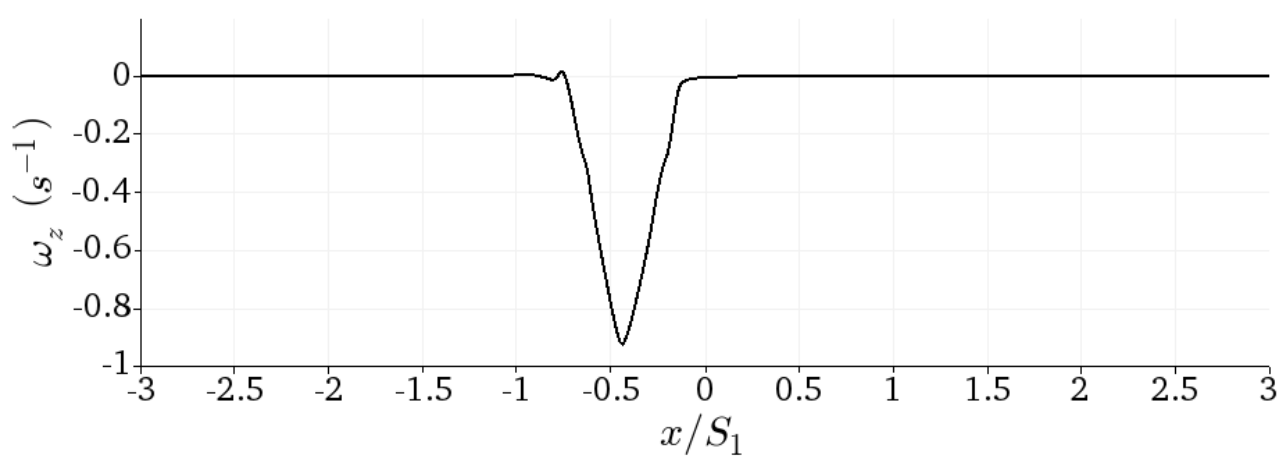

(b) $t U / S_{1} \simeq 1.6$ during the maximum generation phase

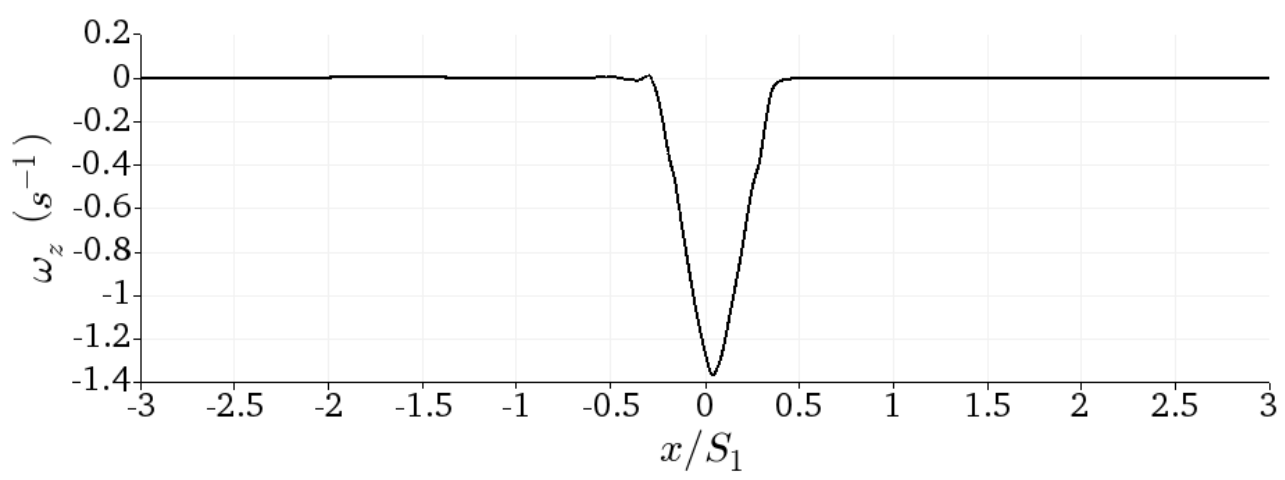

(c) $t U / S_{1} \simeq 2.1$ during the end phase

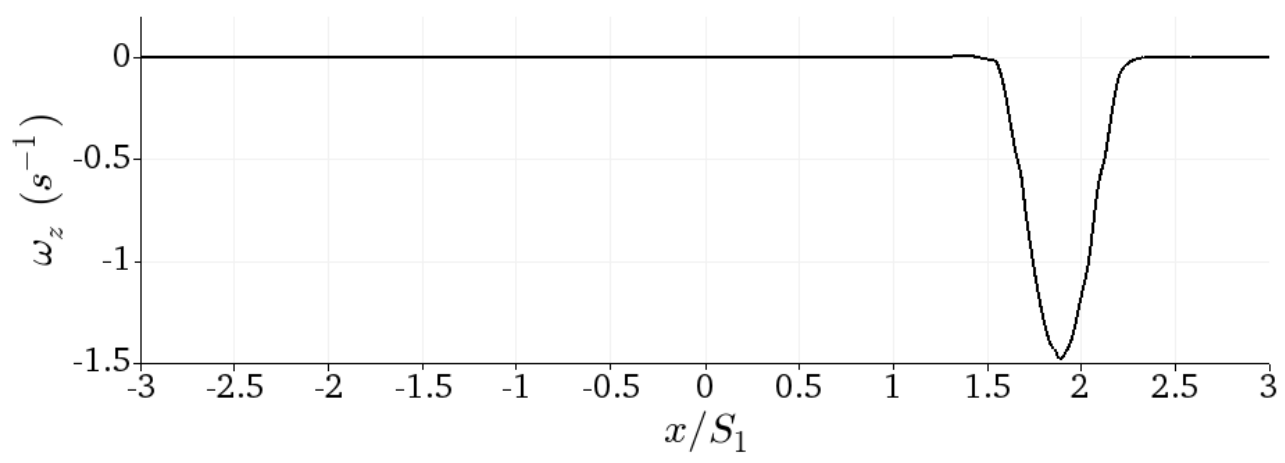

(d) $t U / S_{1} \simeq 4.0$ at full maturity after the completion of the vortex generation phases

Figure 4.14: The vorticity $\omega_{z}$ distribution along a line at $y=0.4 S_{1}$ at different stages of vortex generation for a vortex of $\tilde{\Gamma}=-0.86$. 
Chapter 4 - Vortex-nozzle interaction: two-dimensional plane Euler SIMULATIONS BASED LUMPED ELEMENT SOUND SOURCE MODEL

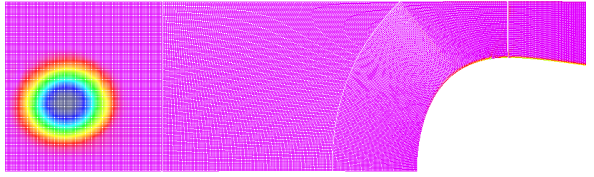

(a) $\left(t-t_{\text {travel }}\right) U / S_{1} \simeq 4.6$

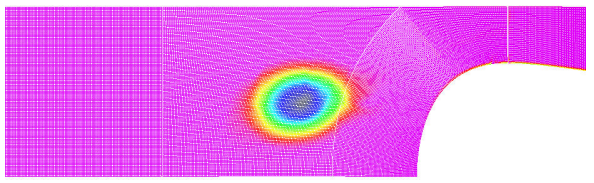

(c) $\left(t-t_{\text {travel }}\right) U / S_{1} \simeq 6$.

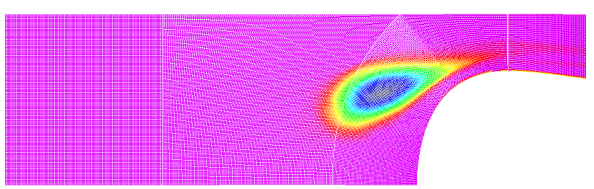

(e) $\left(t-t_{\text {travel }}\right) U / S_{1} \simeq 6.8$

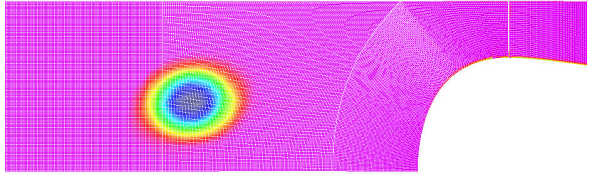

(b) $\left(t-t_{\text {travel }}\right) U / S_{1} \simeq 5.3$

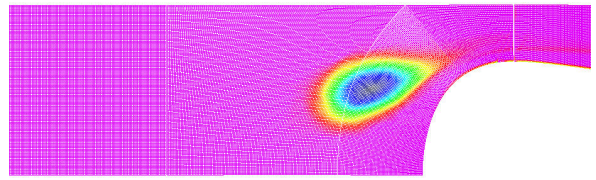

(d) $\left(t-t_{\text {travel }}\right) U / S_{1} \simeq 6.5$

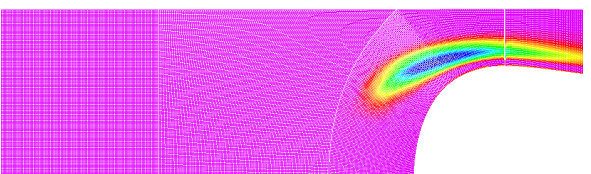

(f) $\left(t-t_{\text {travel }}\right) U / S_{1} \simeq 7.1$

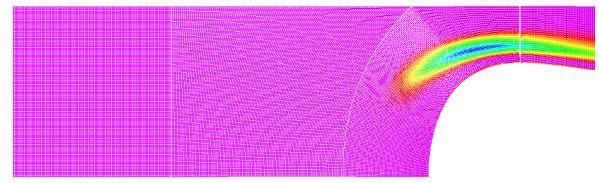

(g) $\left(t-t_{\text {travel }}\right) U / S_{1} \simeq 7.8$

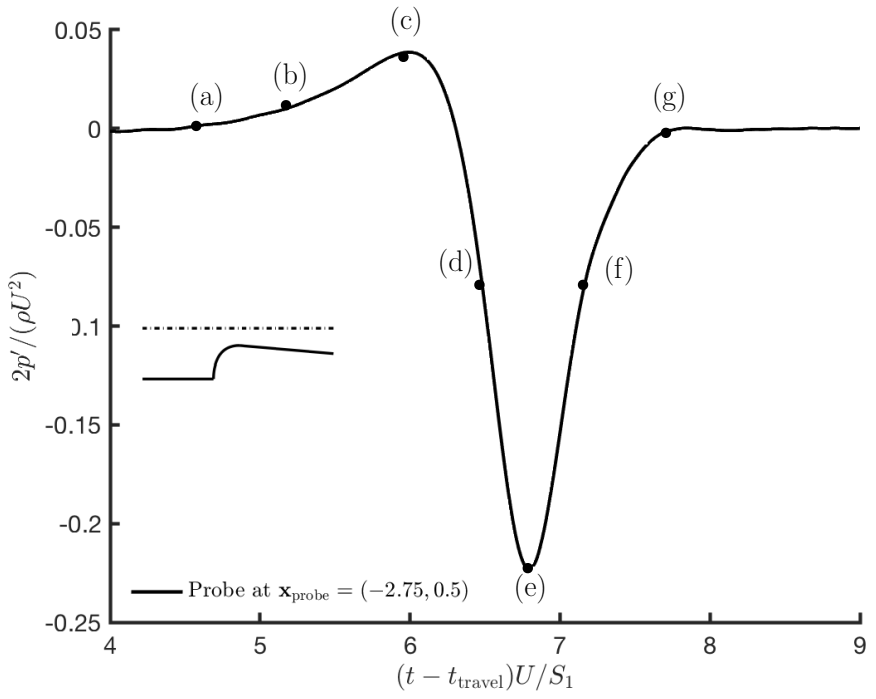

(h) Recorded signal at upstream probe

Figure 4.15: Vortex of $\tilde{\Gamma}=-0.86$ and $R_{\Gamma}=0.3 S_{1}$ passing through a right angle inlet nozzle. The vortex was released from $(-2,0.4) S_{1}$ i.e. the vortex release height used was $h=0.4 S_{1}$. 
Chapter 4 - Vortex-nozzle interaction: two-dimensional plane Euler SIMULATIONS BASED LUMPED ELEMENT SOUND SOURCE MODEL

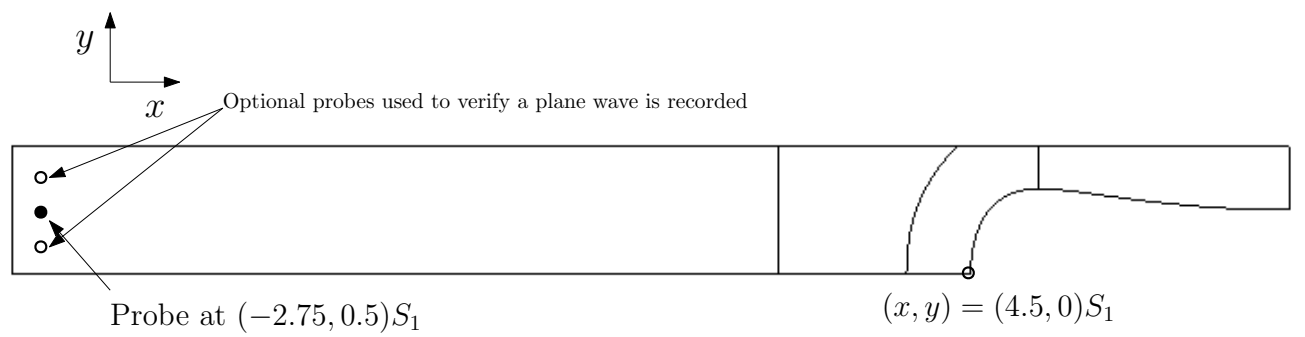

Figure 4.16: Position of the probe for acoustic response recording, the position of the probe is highlighted with a black dot at $(-2.75,0.5) S_{1}$. The acoustic response is a plane wave, thus to verify that a plane wave is being recored two additional probes placed at $(-2.75,0.25) S_{1}$ and $(-2.75,0.75) S_{1}$ these are indicated with the circles.

stationary base flow state. The acoustic response was found to be a plane wave, this was verified using two additional probes are placed at $(-2.75,0.52) S_{1}$ and $(-2.75,0.75) S_{1}$ these are indicated with the open circles in Fig. 4.16.

The travel time, $t_{\text {travel }}$, the time the acoustic wave takes to reach the probe coming from the nozzle, is estimated as follows:

$$
t_{\text {travel }}=\frac{\Delta x_{\text {probe }}}{c-U}
$$

where $\Delta x_{\text {probe }}=7.25 S_{1}$ is the distance from the nozzle inlet $\left(x_{\text {inlet }}=4.5 S_{1}\right)$ to the probe $\left(x_{\text {probe }}=-2.75\right)$. In Fig. $4.15(\mathrm{~h})$ The signal recored $p^{\prime}$ by the probe at $\mathbf{x}_{\text {probe }}=(-2.75,0.5) S_{1}$ scaled by the dynamic pressure $\rho U^{2} / 2$ is shown as a function of $\left(t-t_{\text {travel }}\right) U / S_{1}$. On the signal labels (a), (b),... (g) have been added. These labels correspond to the stages of vortex-nozzle interaction shown in Fig. 4.15 (a), (b), .. (g).

\subsection{Parametric study of vortex-nozzle interaction with nozzle cavity}

The results of parameter studies of vortex-nozzle interaction for an integrated inlet nozzle geometry (Fig. 4.2 (a)) are shown and discussed in this section.

Simulations with varied vortex-core radius $R_{\Gamma}$ have been carried out. These simulations were done with fixed values of the vortex circulation $\tilde{\Gamma}=-0.862$, upstream vortex release height $h=0.5 S_{1}$ and upstream Mach number $M=$ 0.197. The results are shown in Fig. 4.17. In this graph the scaled upstream acoustic response $\frac{2 p^{\prime}}{\rho U^{2} \tilde{\Gamma} M}$ is shown as a function of dimensionless time $t U / S_{1}$. Three vortex-core radii were used $R_{\Gamma} / S_{1}=0.2,0.3$ and 0.4 . The acoustic responses for these three vortex-core radii were found to be qualitatively the same. The deviation of the pulse amplitude maximum between the reference 
Chapter 4 - Vortex-nozzle interaction: two-dimensional plane Euler SIMULATIONS BASED LUMPED ELEMENT SOUND SOURCE MODEL

case $\left(R_{\Gamma}=0.3 S_{1}\right)$ and the case with the largest peak value was less than $17 \%$. Thus, the vortex-core radius for further parameter studies (e.g. variation of the upstream vortex release height $h$ ) was fixed at $R_{\Gamma}=0.3 S_{1}$.

Looking at the first part of the acoustic response (Fig. 4.17), in the time range $6 \leq t U / S_{1} \leq 9$, one recognizes the time derivative of a pulse as shown in Fig. 4.8. For later times $t U / S_{1}>9$ one observes a decaying oscillation, which corresponds to a plane wave generated by the transversal oscillations of the cavity/main duct combination. The acoustic wavelength of this transversal acoustic oscillation is for the given cavity about is $5 S_{1}$. This corresponds to a dimensionless oscillation period $5 S_{1} U /\left(c S_{1}\right)=5 M \simeq 1$ for $M=0.197$. It will be shown later that this dimensionless transversal oscillation period indeed decreases linearly with decreasing Mach number (Fig. 4.21 and 4.22). The quality factor of the oscillation is approximately three. This oscillation decays rapidly because of a plane wave radiation it generates along the main flow channel for this transverse acoustic standing wave with a pressure antinode on the symmetry axis (upper wall in the simulations). In appendix $\mathrm{G}$, a figure similar to Fig. 4.15 shows in the presence of a nozzle cavity sound production occurs on approach to the nozzle as well.

The influence of the of the upstream vortex release height $h$, measured with respect to the lower wall of the upstream channel, has been investigated. This was done using simulations with the following fixed parameters: vortex-core radius $R_{\Gamma}=0.3 S_{1}$, vortex circulation $\tilde{\Gamma}=-1.73$ and upstream Mach number $M=0.197$. The upstream release height $h$ was varied, the results are shown in Fig. 4.18. The solid line is for $h=0.4 S_{1}$, the dashed line is for $h=0.5 S_{1}$ and $h=0.6 S_{1}$. In Fig. 4.18, the scaled upstream acoustic response $\frac{2 p^{\prime}}{\rho U^{2} \tilde{\Gamma} M}$ is shown as a function of dimensionless time $t U / S_{1}$. One observes that the scaled upstream acoustic response $\frac{2 p^{\prime}}{\rho U^{2} \tilde{\Gamma} M}$ is qualitatively the same for the three release heights. The difference in peak amplitudes between results is less than $15 \%$. Overall, one can conclude that the upstream acoustic response due to vortexnozzle interaction is not sensitive to variation of the vortex release height for $0.4 \leq h / S_{1} \leq 0.6$. This confirms the assumption that small deviations in vortex path will not influence the sound production, when it is dominated by the cavity response. Hence, in the remaining results are shown for fixed upstream release height $h=0.4 S_{1}$.

The influence of dimensionless vortex circulation $\tilde{\Gamma}=\frac{\Gamma}{S_{1} U}$ was investigated with the fixed vortex-core radius $R_{\Gamma}=0.3 S_{1}$, upstream Mach number $M=0.197$ and release height $h=0.4 S_{1}$. Simulations with four vortex circulations were carried out, $\tilde{\Gamma}=-0.108, \tilde{\Gamma}=-0.860, \tilde{\Gamma}=-1.20$ and $\tilde{\Gamma}=-1.73$. The results are shown in Fig. 4.19. The dimensionless upstream acoustic response is qualitatively the same for these vortex circulations. The most pronounced 


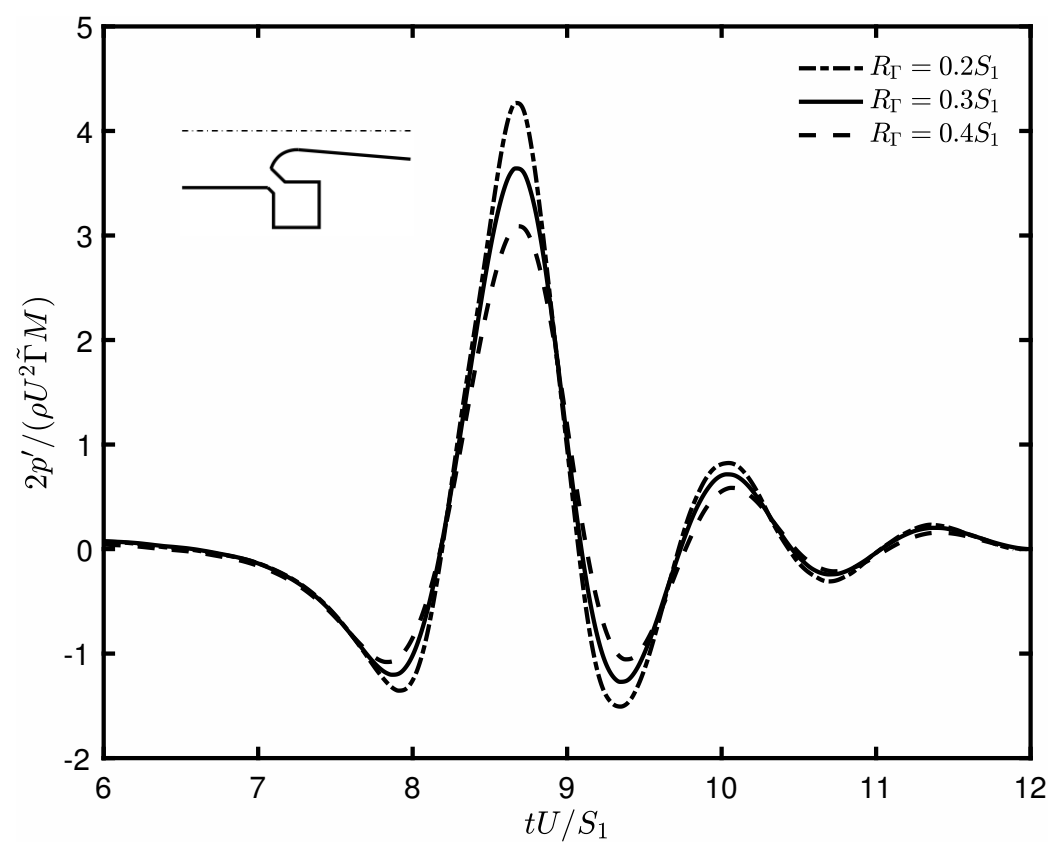

Figure 4.17: Influence of vortex-core radius $R_{\Gamma}$. The scaled upstream acoustic response $\frac{2 p^{\prime}}{\rho U^{2} \tilde{\Gamma} M}$ due to vortex-nozzle interaction as a function of dimensionless time $t U / S_{1}$ is shown in this figure. Three vortex-core radii were used $R_{\Gamma}=0.2 S_{1}$ (unevenly dashed line), $0.3 S_{1}$ (solid line) and $0.4 S_{1}$ (dashed line). One observes that the upstream acoustic response is qualitatively the same for these three vortex-core radii. These results were obtained with an upstream vortex release height $h=0.5 S_{1}$, vortex circulation $\tilde{\Gamma}=-0.862$ and upstream Mach number $M=0.197$. 
Chapter 4 - Vortex-nozzle interaction: two-dimensional plane Euler SIMULATIONS BASED LUMPED ELEMENT SOUND SOURCE MODEL

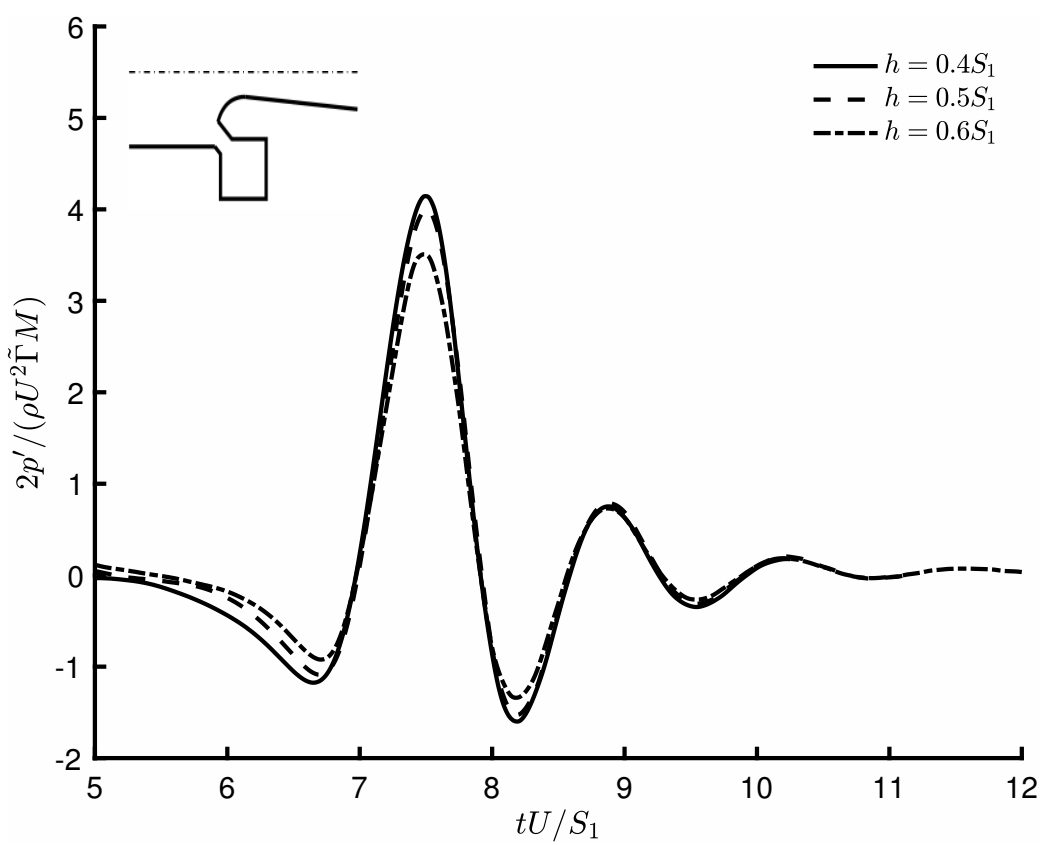

Figure 4.18: Influence of varied upstream release height $h$. The scaled upstream acoustic response $\frac{2 p^{\prime}}{\rho U^{2} \tilde{\Gamma} M}$ due to vortex-nozzle interaction as a function of dimensionless time $t U / S_{1}$ is shown. Three upstream release heights were used $h=0.4 S_{1}$ ( solid line), $h=0.5 S_{1}$ (dashed line) and $h=0.6 S_{1}$ (unevenly dashed line). One observes that the upstream acoustic response is qualitatively the same for these three release heights. These results were obtained with an upstream Mach number $M=0.197$, vortex-core radius $R_{\Gamma}=0.3 S_{1}$ and vortex circulation $\tilde{\Gamma}=-1.73$. 
Chapter 4 - Vortex-nozzle interaction: two-dimensional plane Euler SIMULATIONS BASED LUMPED ELEMENT SOUND SOURCE MODEL

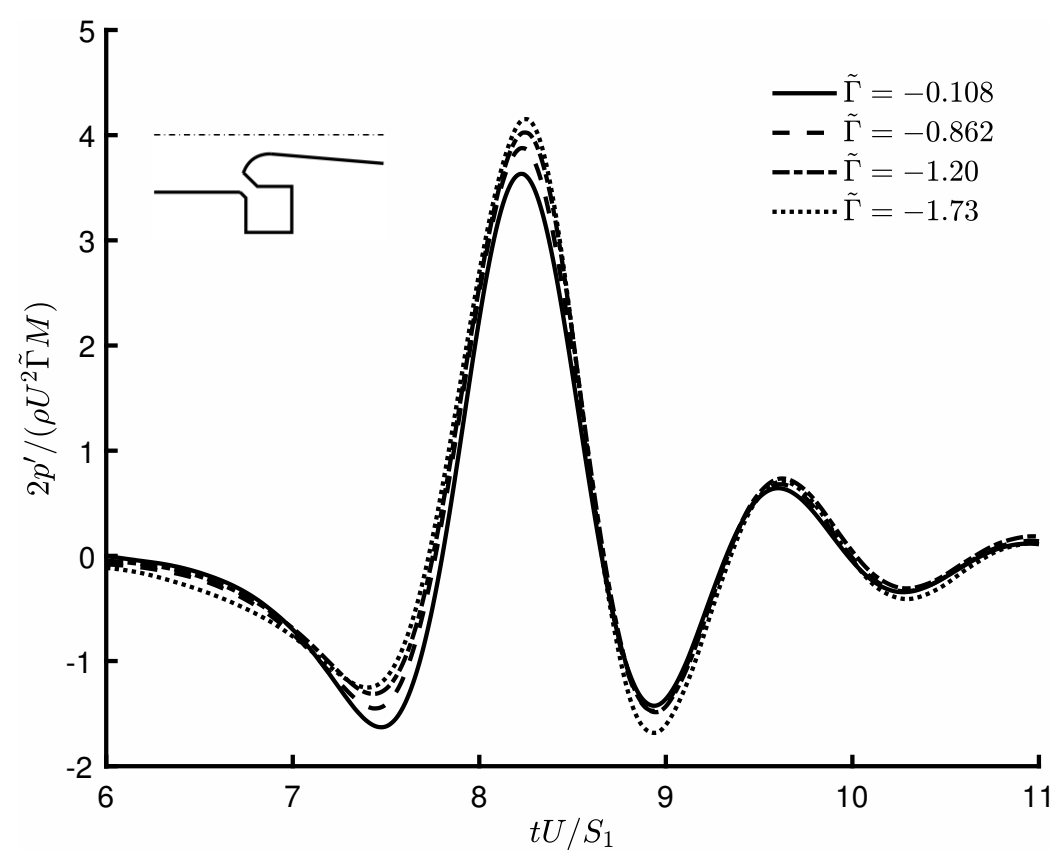

Figure 4.19: Influence dimensionless vortex circulation $\tilde{\Gamma}$. The scaled upstream acoustic response $\frac{2 p^{\prime}}{\rho U^{2} \tilde{\Gamma} M}$ due to vortex-nozzle interaction, as a function of dimensionless time $t U / S_{1}$. The influence of dimensionless vortex circulation $\tilde{\Gamma}=\Gamma / U S_{1}$ was investigated. Four vortex circulations were used $\tilde{\Gamma}=-0.108$ (solid line), -0.860 (dashed line), -1.20 (unevenly dashed line) and -1.73 (finely dashed line). One observes that the upstream acoustic response is qualitatively the same for these vortex circulations. These results were obtained with a vortex-core radius $R_{\Gamma}=0.3 S_{1}$, upstream release height $h=0.4 S_{1}$ and upstream Mach number $M=0.197$.

deviation in response peak is $14 \%$ between $\tilde{\Gamma}=-0.108$ and $\tilde{\Gamma}=-1.73$. This confirms that in the presence of a large cavity volume, $V_{c}$, the sound production is dominated by the compressibility effect, and depends linearly on $\tilde{\Gamma}$.

While the vortex circulation $\tilde{\Gamma}$ is negative in the actual flow, it is interesting to compare the response for vortex circulations of opposite sign. In Fig. 4.20 the results for $\tilde{\Gamma}= \pm 0.108$ are shown. A deviation of approximately $10 \%$ in peak value for $\tilde{\Gamma}=-0.108$ (solid line) and $\tilde{\Gamma}=0.108$ (dashed line) is observed.

To investigate the influence of the upstream Mach number, $M$, on the acoustic response, simulations with $M=0.058,0.097$ and 0.197 have been carried out. This implies a change in nozzle contraction ratio in as explained in section 4.6.3. It has been observed that the signal due to vortex-nozzle interaction is proportional $M$ as expected from theory. Results of simulations for $|\tilde{\Gamma}| \simeq 0.1$, are shown in Fig. 4.20 and 4.21 and for $|\tilde{\Gamma}| \simeq 0.9$ in Fig. 4.22. In Fig. 4.21 and Fig. 4.22 the scaled acoustic response $2 p^{\prime} /\left(\rho U^{2} \tilde{\Gamma} M\right)$ is shown as a function 
Chapter 4 - Vortex-nozzle interaction: two-dimensional plane Euler SIMULATIONS BASED LUMPED ELEMENT SOUND SOURCE MODEL

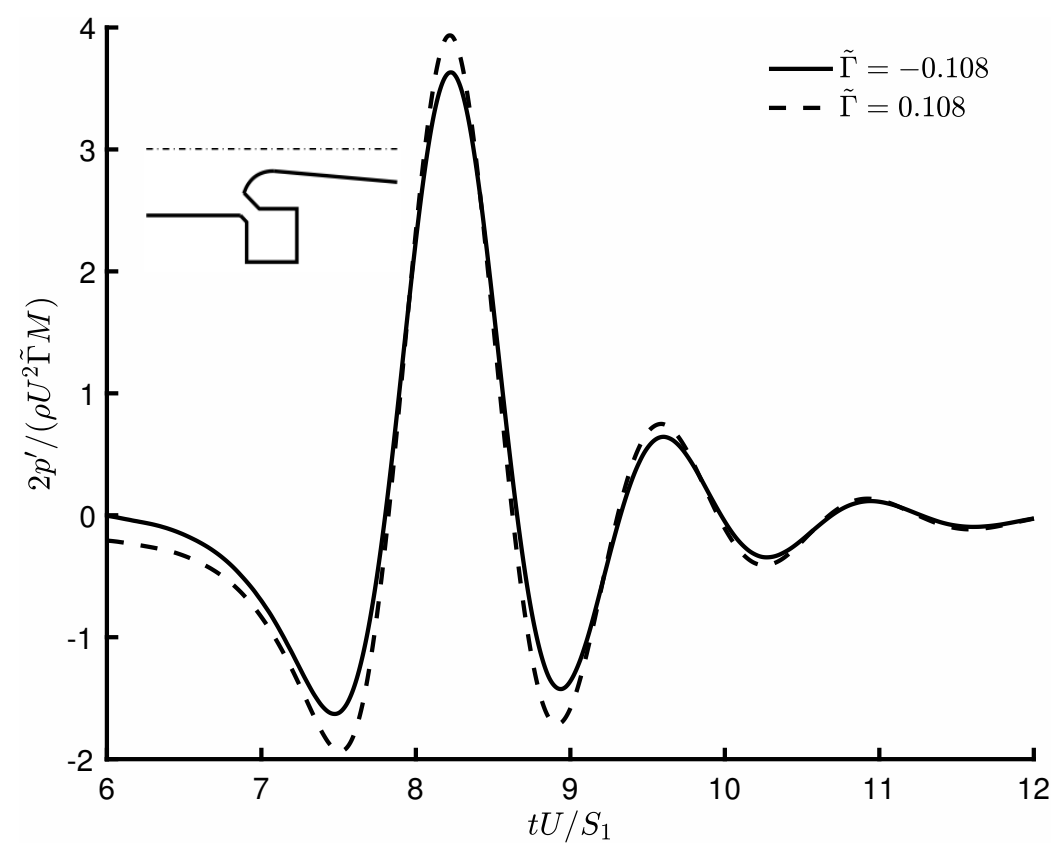

Figure 4.20: Influence of the sign of the circulation. The scaled upstream acoustic response $\frac{2 p^{\prime}}{\rho U^{2} \tilde{\Gamma} M}$ due to vortex-nozzle interaction for $\tilde{\Gamma}= \pm 0.108$ as a function of dimensionless time $t U / S_{1}$. In this graph $\tilde{\Gamma}=-0.108, \tilde{\Gamma}=108$ correspond to the solid and dashed lines respectively. The upstream acoustic response displays qualitatively the same behavior for these vortex circulations. These results were obtained with upstream Mach number $M=0.197$, vortex release height $h=0.4 S_{1}$ and vortex-core radius $R_{\Gamma}=0.3 S_{1}$. 
of the dimensionless time $t U / S_{1}$. As was alluded to earlier, the transversal cavity-channel mode of oscillation at a frequency of the order $c /\left(5 S_{1}\right)$ has a dimensionless frequency that increases as $1 /(5 M)$ with decreasing Mach number. Furthermore, as the dimensionless pulse width is fixed, but decreases in reality with increasing upstream velocity $U=M c$, the relative position of the transversal resonance frequency shifts with respect to the excitation pulse spectrum. This is illustrated in Fig. 4.23. As a consequence, the amplitude of the transversal mode oscillation decreases with decreasing Mach number. This is observed in Fig 4.21 and 4.22. In the following discussion the first part of the acoustic response is focused on. The largest observed deviation of approximately $15 \%$ is between the peak value for $M=0.197$ and $M=0.097$. In Fig. 4.21 , the influence of the upstream Mach number $M$ on the scaled acoustic response $2 p^{\prime} /\left(\rho U^{2} \tilde{\Gamma} M\right)$ is shown as a function of dimensionless time $t U / S_{1}$. The results in Fig. 4.21 were obtained for $h=0.4 S_{1}, R_{\Gamma}=0.3 S_{1}$ and $\tilde{\Gamma} \simeq-0.1$. In Fig. 4.21, the solid line is for $M=0.197$, the dashed line $M=0.097$ and the dotted line $M=0.058$. The largest deviation between the results for $\tilde{\Gamma} \simeq-0.1$ is $29 \%$. One concludes that in the presence of a cavity the pressure pulse amplitude scales as $\rho U^{2} \tilde{\Gamma} M / 2$.

\subsection{Parametric study of vortex-nozzle interaction for the right angle nozzle inlet without cavity}

The results of parameter studies of vortex-nozzle interaction for a right angle inlet nozzle geometry are shown and discussed in this section.

Simulations with a fixed upstream Mach number $M=0.197$, release height $h=0.5 S_{1}$ and vortex circulation $\tilde{\Gamma}=-0.858$ were carried out to investigate the influence of the vortex-core radius $R_{\Gamma}$. In Fig. 4.24 results for simulations with $R_{\Gamma}=0.2 S_{1}, 0.3 S_{1}$ and $0.4 S_{1}$ are shown. One observes that the upstream acoustic response is qualitatively the same for these three vortex-core radii. There is a $30 \%$ deviation between the peak values of the acoustic response for $R_{\Gamma}=0.4 S_{1}$ and $R_{\Gamma} / S_{1}=0.2$ relative to the case where $R_{\Gamma}=0.3 S_{1}$. This significantly larger than for the case of the nozzle with cavity (Fig. 4.17), but it is still relatively small. Before the acoustic response peak, a less pronounced pressure dip, than in the case of a large cavity is observed. The presence of this dip indicates that there are still some compressibility effects acting a an residual cavity volume in terms of the lumped element model. This residual volume $V_{0}$ will be discussed in section 4.9. The oscillations of the transversal mode after the acoustic response peak are drastically reduced and have shifted to the high dimensionless frequency $1 /(2 M)$ (transversal mode of the main channel). With respect to the case with the cavity volume (section 4.7), the acoustic response peak value has been reduced by a factor three. 
Chapter 4 - Vortex-nozzle interaction: two-dimensional plane Euler SIMULATIONS BASED LUMPED ELEMENT SOUND SOURCE MODEL

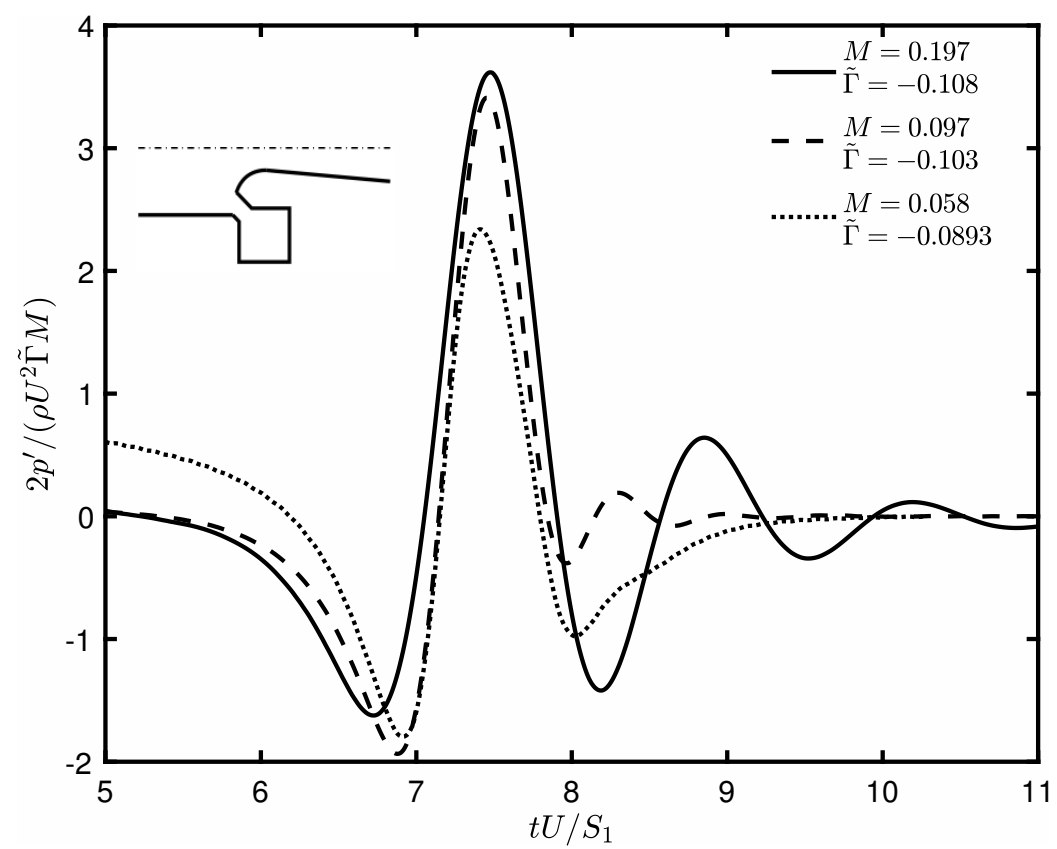

Figure 4.21: Influence of the upstream Mach number $M$ for $\tilde{\Gamma} \simeq-0.1$. Vortex nozzle interaction simulation with three upstream Mach numbers $M=\{0.197,0.097,0.058\}$ (solid, dashed and dotted lines respectively) with $\tilde{\Gamma} \simeq-0.1$ were carried out. A linear dependence of the Mach number on the signal was found. In this figure the scaled acoustic response $\frac{2 p^{\prime}}{\rho U^{2} \tilde{\Gamma} M}$ as a function of dimensionless time $t U / S_{1}$ is shown. These results were obtained with upstream vortex release height $h=0.4 S_{1}$, vortex-core radius $R_{\Gamma}=0.3 S_{1}$. 
Chapter 4 - Vortex-nozzle interaction: two-dimensional plane Euler SIMULATIONS BASED LUMPED ELEMENT SOUND SOURCE MODEL

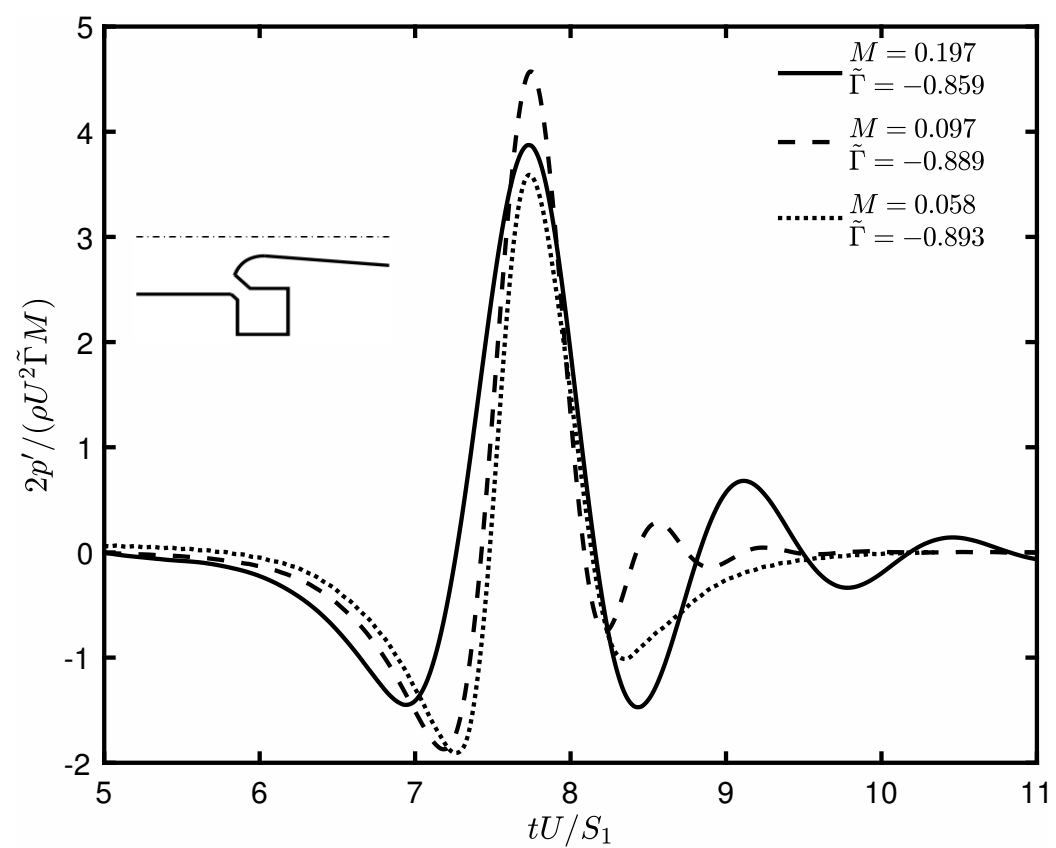

Figure 4.22: Influence of the upstream Mach number $M$ for $\tilde{\Gamma} \simeq-0.9$. Vortex nozzle interaction simulation with three upstream Mach numbers $M=\{0.197,0.097,0.058\}$ were carried out. A linear dependence of the Mach number on the signal was found. In this figure the scaled acoustic response $\frac{2 p^{\prime}}{\rho U^{2} \tilde{\Gamma} M}$ as a function of dimensionless time $t U / S_{1}$ is shown. These results were obtained with upstream vortex release height $h=$ $0.4 S_{1}$, vortex-core radius $R_{\Gamma}=0.3 S_{1}$.

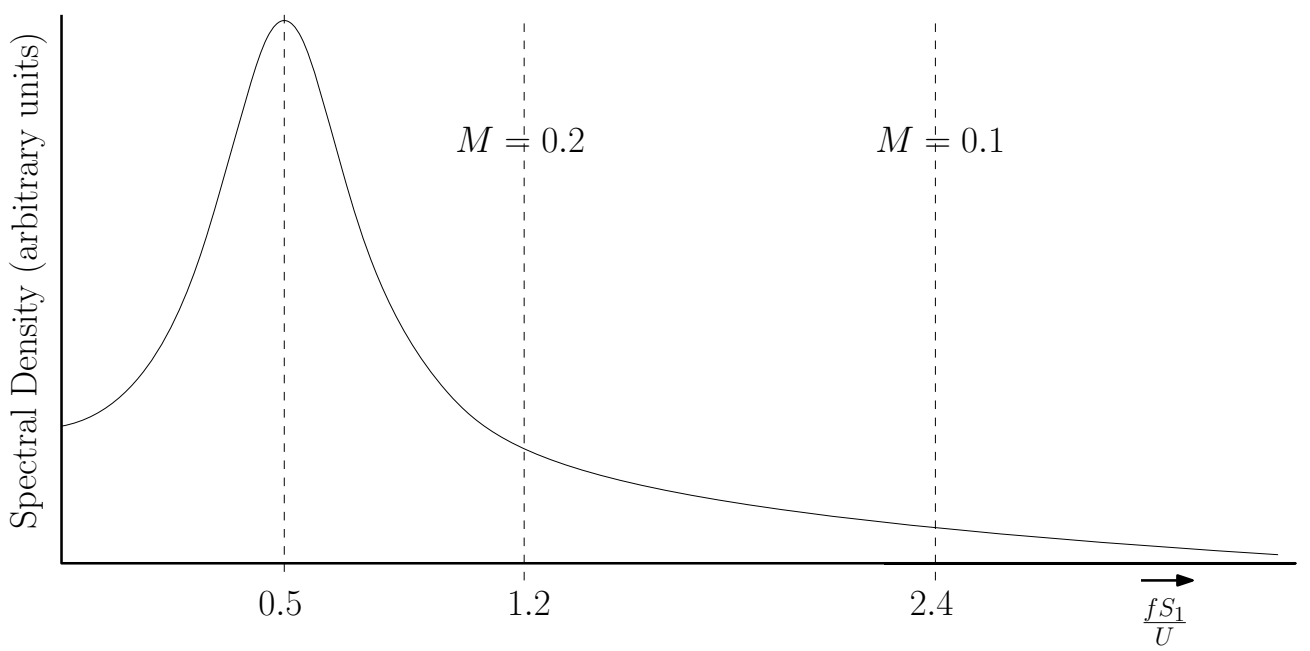

Figure 4.23: Sketch of transversal mode frequencies in spectrum. The dimensionless transversal cavity-channel mode oscillation frequency shifts away from the excitation spectrum maximum with decreasing Mach number $M$. This decreases the transversal wave excitation amplitude with decreasing Mach number. 
Chapter 4 - Vortex-nozzle interaction: two-dimensional plane Euler SIMULATIONS BASED LUMPED ELEMENT SOUND SOURCE MODEL

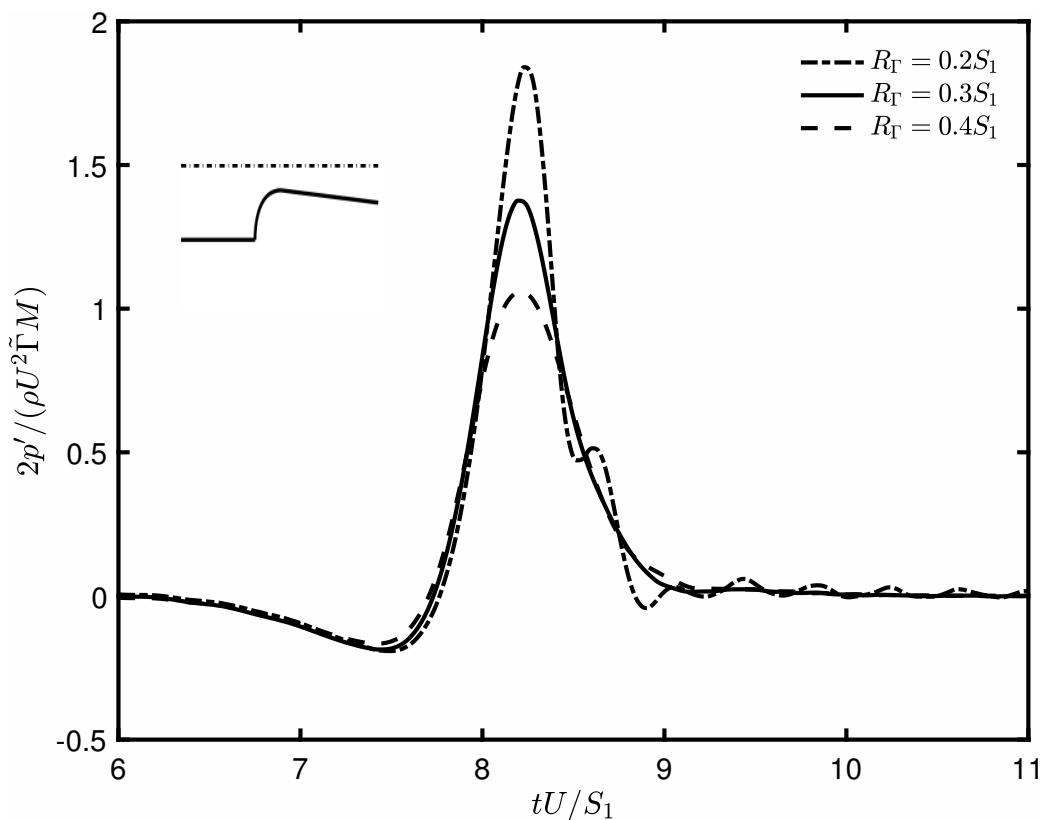

Figure 4.24: Influence of vortex-core radius $R_{\Gamma}$. The scaled upstream acoustic response $2 p^{\prime} /\left(\rho U^{2} \tilde{\Gamma} M\right)$ due to vortex-nozzle interaction for three vortex-radii $R_{\Gamma}=0.2 S_{1}$ (unevenly dashed line), $R_{\Gamma}=0.3 S_{1}$ (solid line) and $R_{\Gamma}=0.4 S_{1}$ (dashed line) as a function of dimensionless time $t U / S_{1}$ is shown. The upstream acoustic response is qualitatively the same for these three vortex-core radii. There is a $30 \%$ deviation between the peak values of the acoustic response for $R_{\Gamma}=0.4 S_{1}$ and $R_{\Gamma}=0.2 S_{1}$ relative to the case for $R_{\Gamma}=0.3 S_{1}$. These results were obtained with vortex circulation $\tilde{\Gamma}=-0.858$, upstream vortex release height $h=0.5 S_{1}$ and Mach number $M=0.197$. 
The influence of the vortex upstream release height $h$ (measured from the lower channel wall to the center of the vortex) on the acoustic response has been investigated. Simulations with three release heights $h=0.4 S_{1}, 0.5 S_{1}$ and $0.6 S_{1}$ have been carried out. For these simulations, the following parameters have been fixed: the dimensionless vortex circulation $\tilde{\Gamma}=-1.72$, the vortex-core radius $R_{\Gamma}=0.3 S_{1}$ and the upstream Mach number $M=0.197$. The results are shown in Fig. 4.25. One observes that the upstream acoustic response is qualitatively the same for the three release heights. The deviation between the peak values of the acoustic response for $h=0.4 S_{1}$ and $h=0.6 S_{1}$ are within $10 \%$ of relative to the case for $h=0.5 S_{1}$. One concludes that overall, the upstream acoustic response due to vortex-nozzle interaction is not influenced significantly for release heights in the range $0.4 \leq h / S_{1} \leq 0.6$. This implies that the acoustic response is not strongly dependent on the vortex path. This indicates that the compressibility of the gas around the nozzle inlet is important. This effect will be represented by an effective cavity volume $V_{\text {eff }}=V_{c}+V_{0}$. Where $V_{0}$ is the residual volume, which accounts for the compressibility of the gas around the inlet of the nozzle when $V_{c}=0$.

The influence of the dimensionless vortex circulation, $\tilde{\Gamma}$, including its sign have been investigated for a right angle inlet geometry. In Fig. 4.26 results of vortexnozzle interaction simulations for fixed Mach number $M=0.197$, vortex-core radius $R_{\Gamma}=0.3 S_{1}$ and upstream vortex release height $h=0.4 S_{1}$ are shown. The scaled acoustic response $2 p^{\prime} /\left(\rho U^{2} \tilde{\Gamma} M\right)$ is shown as a function of the dimensionless time $t U / S_{1}$ in Fig. 4.26. The thin lines in Fig. 4.26 are for vortex circulations $\tilde{\Gamma}= \pm 0.107$ and $\tilde{\Gamma}= \pm 0.429$. The negative values fall within the estimated vortex circulation range $-1 \leq \tilde{\Gamma} \leq-0.1$ discussed in section 4.2.3. The thick lines are for vortex circulations $\overline{\tilde{\Gamma}}=1.72$ and $\tilde{\Gamma}=-1.72$, which fall outside of the estimated range of vortex circulations (section 4.2.3). For $\tilde{\Gamma}= \pm 0.107$, the thin dashed and solid line respectively, one observes that the scaling results in similar peak values of the acoustic response. When the absolute value of the circulation is increased to $|\tilde{\Gamma}|=0.429$ a deviation of the peak values of approximately $40 \%$ is observed. The emergence of a fluctuation after the positive peak around $t U / S_{1}=8.75$ on the signal for vortex circulation $\tilde{\Gamma}=0.429$ is observed. When the vortex circulation strength is further increased to either $\tilde{\Gamma}= \pm 1.72$ (the thick solid line and dashed line in Fig. 4.26 , respectively) it is observed that the qualitative behavior of the acoustic response is drastically different. This is interpreted to mean that for the case of a right angle nozzle inlet geometry, the linear scaling of acoustic response with respect to the vortex circulation $\tilde{\Gamma}$ of the acoustic response no longer applies for $|\tilde{\Gamma}|>1$. For $|\tilde{\Gamma}|=1.72$ transitional behavior to a quadratic scaling is observed, as predicted by both the incompressible model (Hirschberg et al. 2017) (chapter 3) or the lumped model without cavity. In this transitional regime the linear and quadratic contributions are of the same order of magnitude. 
Chapter 4 - Vortex-nozzle interaction: two-dimensional plane Euler SIMULATIONS BASED LUMPED ELEMENT SOUND SOURCE MODEL

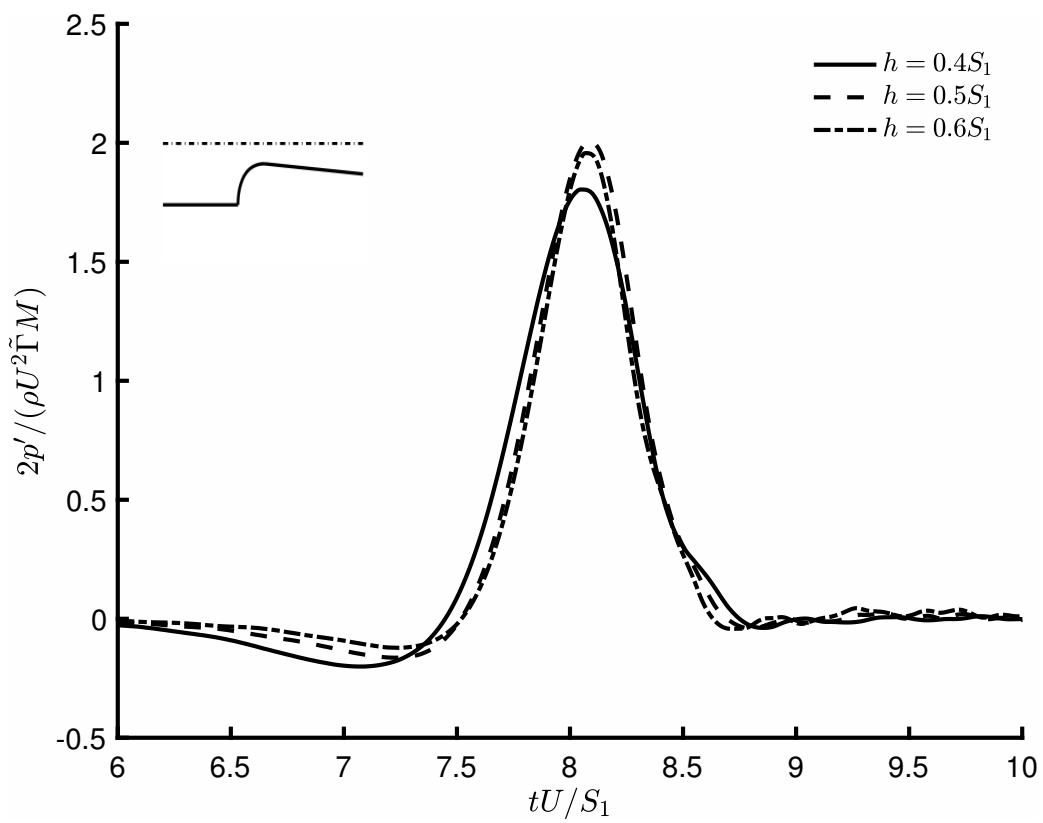

Figure 4.25: Influence of upstream vortex release height $h$ for $\tilde{\Gamma}=-1.72$. The scaled upstream acoustic response $2 p^{\prime} /\left(\rho U^{2} \tilde{\Gamma} M\right)$ due to vortex-nozzle interaction for upstream vortex release heights $h=0.4 S_{1}$ (solid line), $h=0.5 S_{1}$ (dashed line) and $h=0.6 S_{1}$ (unevenly dashed line) as a function of dimensionless time $t U / S_{1}$ is shown. One notices that the upstream acoustic response is qualitatively the same for these three release heights. The deviation between the peak values of the acoustic response for $h=0.4 S_{1}$ and $h=0.6 S_{1}$ is $10 \%$ vis-à-vis the case for $h=0.5 S_{1}$. One concludes that overall the upstream acoustic response due to vortex-nozzle interaction is not influenced significantly for $0.4 \leq h / S_{1} \leq 0.6$. These results were obtained with upstream Mach number $M=0.197$, vortex release height $h=0.4 S_{1}$ and vortex circulation $\tilde{\Gamma}=-1.72$. 


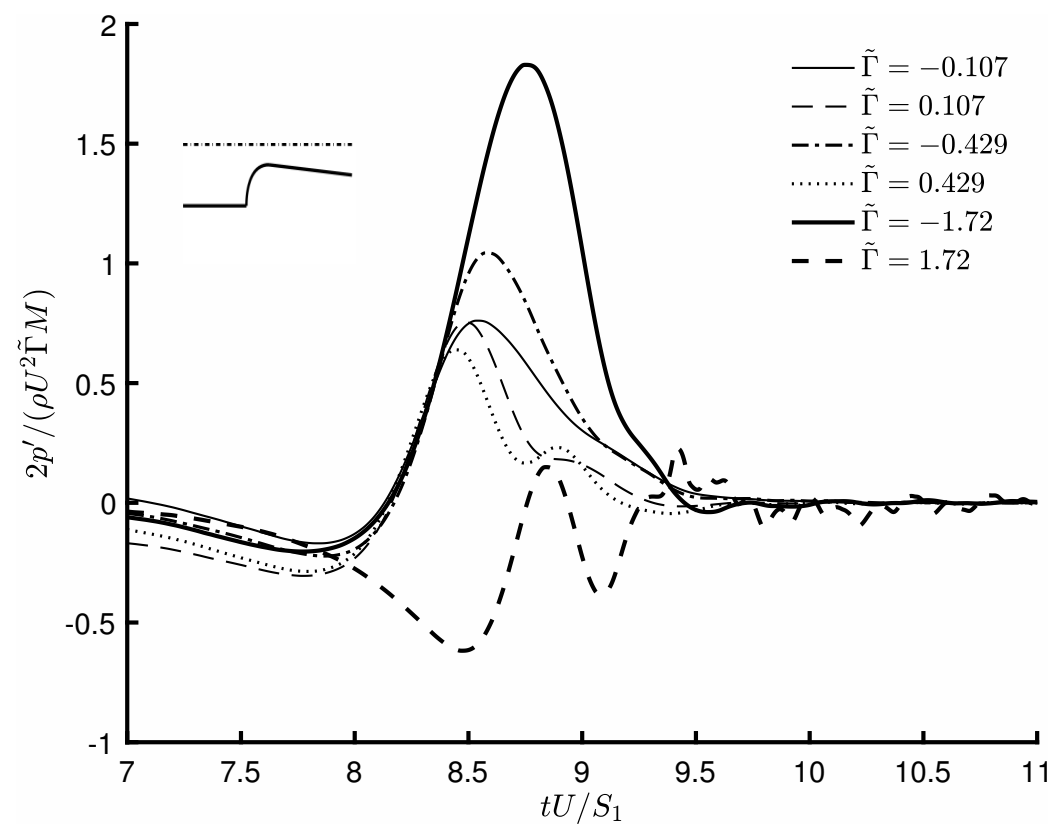

Figure 4.26: Influence vortex circulation $\tilde{\Gamma}$. The scaled upstream acoustic response $2 p^{\prime} /\left(\rho U^{2} \tilde{\Gamma} M\right)$ due to vortex-nozzle interaction for varied vortex circulation $\tilde{\Gamma}$ as a function of dimensionless time $t U / S_{1}$ is shown. The thin lines in this graph are for vortex circulations $\tilde{\Gamma}= \pm 0.107$ and $\tilde{\Gamma}= \pm 0.429$, the negative values fall within the estimated vortex circulation range $-1 \leq \tilde{\Gamma} \leq-0.1$. The thick lines are for vortex circulations $\tilde{\Gamma}=1.72$ and $\tilde{\Gamma}=-1.72$, which fall outside of the estimated range of vortex circulations. These results were obtained with upstream Mach number $M=0.197$, vortex release height $h=0.4 S_{1}$ and vortex-core radius $R_{\Gamma}=0.3 S_{1}$. 
Chapter 4 - Vortex-nozzle interaction: two-dimensional Plane Euler SIMULATIONS BASED LUMPED ELEMENT SOUND SOURCE MODEL

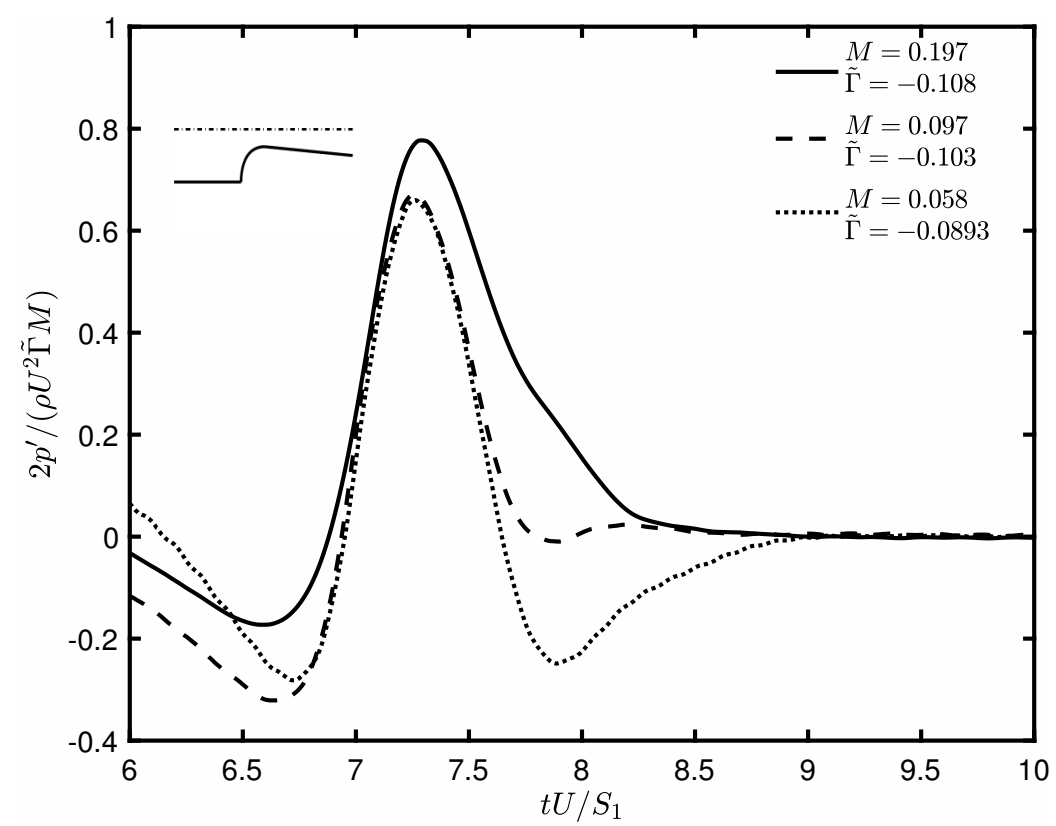

Figure 4.27: Influence upstream Mach number $M$ for vortex circulation $\tilde{\Gamma} \simeq-0.1$. The influence of the upstream Mach number $M$ was investigated. Vortex nozzle interaction simulation with three upstream Mach numbers $M=\{0.197,0.097,0.058\}$ were carried out. A linear dependence of the Mach number on the signal was found. In this figure the scaled acoustic response $2 p^{\prime} / \rho U^{2} \tilde{\Gamma} M$ as a function of dimensionless time $t U / S_{1}$ is shown.

Finally, the influence of the upstream Mach number $M$ on the acoustic response due to vortex-nozzle interaction for a right angle inlet geometry has also been investigated. In Fig. 4.27 the scaled acoustic response $\frac{2 p^{\prime}}{\rho U^{2} \tilde{\Gamma} M}$ as a function of dimensionless time $t U / S_{1}$ obtained with vortex circulation $\tilde{\Gamma} \simeq-0.1$, upstream release height $h=0.4 S_{1}$ and vortex-core radius $R_{\Gamma}=0.3 S_{1}$ is shown for $M=0.197,0.097$ and 0.058 . In Fig. 4.27 for $\tilde{\Gamma} \simeq-0.1$ the qualitative behavior is broadly the same for the three Mach numbers. A deviation of approximately $14 \%$ between the peak value for $M=0.197$ and the other two upstream Mach numbers obtained with $\tilde{\Gamma} \simeq-0.1$ is observed.

In Fig. 4.28 the influence of the upstream Mach number is investigated as well but for vortex circulation $\tilde{\Gamma} \simeq-0.9$. In Fig. 4.28 the scaled acoustic response $2 p^{\prime} /\left(\rho U^{2} \tilde{\Gamma} M\right)$ as a function of dimensionless time $t U / S_{1}$ is shown for fixed upstream release height $h=0.4 S_{1}$, vortex-core radius $R_{\Gamma}=0.3 S_{1}$ for $M=0.197,0.097$ and 0.058. Comparing the results obtained with $\tilde{\Gamma} \simeq-0.9$, one notices a deviation of approximately $14 \%$ between the peak value for $M=$ 0.197 and the other two upstream Mach numbers. The difference between the results obtained for $\tilde{\Gamma} \simeq-0.1$ is approximately a factor two lower than for $\tilde{\Gamma} \simeq-0.9$. 
Chapter 4 - Vortex-nozzle interaction: two-dimensional plane Euler SIMULATIONS BASED LUMPED ELEMENT SOUND SOURCE MODEL

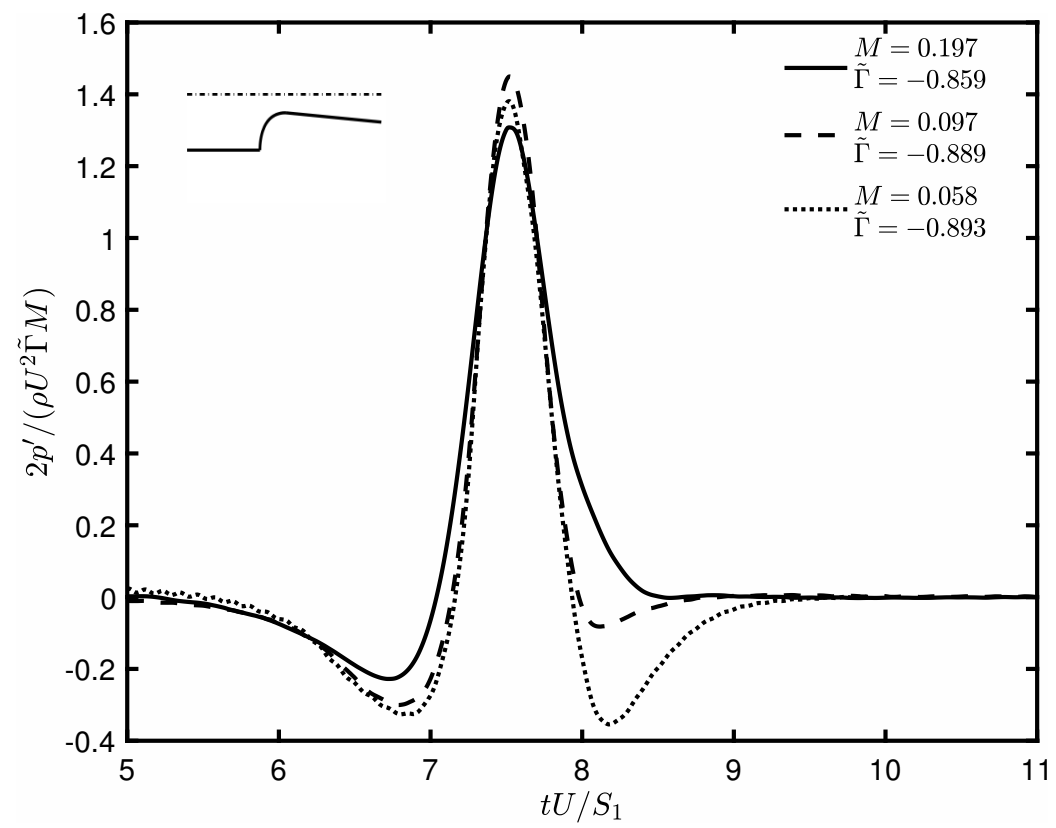

Figure 4.28: Influence upstream Mach number $M$ for vortex circulation $\tilde{\Gamma} \simeq$ -0.9. The influence of the upstream Mach number $M$ was investigated. Vortex nozzle interaction simulation with three upstream Mach numbers $M=\{0.197,0.097,0.058\}$ were carried out. A linear dependence of the Mach number on the signal was found. In this figure the scaled acoustic response $2 p^{\prime} /\left(\rho U^{2} \tilde{\Gamma} M\right)$ as a function of dimensionless time $t U / S_{1}$ is shown. 
Chapter 4 - Vortex-nozzle interaction: two-dimensional plane Euler SIMULATIONS BASED LUMPED ELEMENT SOUND SOURCE MODEL

\subsection{Effective volume $\tilde{V}_{\text {eff }}$}

When vortex-nozzle interaction simulations are visualized it is observed that a upstream-traveling-plane acoustic wave is produced due to compression of gas on the wall of the nozzle inlet. This process is visualized in Fig. 4.29, for a nozzle inlet without nozzle cavity.

This compression effect can be taken into account in the lumped model as a residual cavity volume $V_{0}$ or dimensionless residual length $\tilde{L}_{0}=V_{0} /\left(4 S_{1}^{3}\right)$. The dimensionless effective length $\tilde{L}_{\text {eff }}$ for the proposed lumped element then becomes $\tilde{L}_{\text {eff }}=\tilde{L}_{c}+\tilde{L}_{0}$. To estimate $\tilde{L}_{0}$, simulations for a right angle inlet geometry and an integrated nozzle with cavity volume $V_{c}$ geometry were carried out. These simulations had the same upstream Mach number $M$, vortex release height $h$, vortex circulation $\tilde{\Gamma}$ and vortex-core radius $R_{\Gamma}$. In order to minimize the $\Delta B_{r}$ effect, the simulations were carried out for $\tilde{\Gamma}=-0.1$. In Fig. 4.30 a sketch of the estimation procedure is given. The horizontal axis shows dimensionless length $\tilde{L}_{c}=V_{c} /\left(4 S_{1}^{3}\right)$ and the vertical axis the dimensionless acoustic response peak value $\tilde{p}^{\prime}=\frac{2 p_{\text {peak }}^{\prime}}{\rho U^{2} \tilde{\Gamma}}$. A straight line with slope $\Delta \tilde{p}^{\prime} / \Delta \tilde{L}=\left(\tilde{p}_{\text {cavity }}^{\prime}-\tilde{p}_{\text {sharp }}^{\prime}\right) / \tilde{L}_{c}$ line is used to find $\tilde{L}_{0}=\tilde{p}_{\text {sharp }}^{\prime} /\left(\Delta \tilde{p}^{\prime} / \Delta \tilde{L}\right)$ by extrapolation (where $\tilde{L}_{0}>0$ by definition).

The influence of the upstream release height $h$ for $\tilde{\Gamma} \simeq-0.1$ on the residual length $L_{0}$ has been determined for upstream Mach numbers that are in the range $0.05 \leq M \leq 0.2$ of interest, the results are shown in Fig. 4.31. From Fig. 4.31 one determines that using $\tilde{L}_{0}=0.2$ is a fair approximation for the residual compressibility length $\tilde{L}_{0}$ making $\tilde{L}_{\text {eff }}=0.9$. The value $\tilde{L}_{0}=0.2$ will be used to perform data fits of vortex-nozzle interaction simulations with the proposed lumped element model (section 4.10). In Fig. 4.32 it is shown that scaling the acoustic response due to vortex nozzle interaction for cases involving sharp angle inlet and nozzle cavity geometries results in a fair collapse of the results. In the following, scaling will thus be done with $\rho U^{2} \tilde{\Gamma} M \tilde{L}_{\text {eff }}$.

\subsection{Fit of data using lumped element model}

In the lumped element model of section 4.5, the following time dependent sound sources are considered:

$$
\Delta B_{c}^{\prime}(t)=\left\{\begin{array}{l}
\alpha \tilde{\Gamma} \frac{U^{2}}{2} \sin ^{2}(\omega t) \text { for } 0 \leq \omega t \leq 2 \\
0 \text { for all other } \omega t
\end{array}\right.
$$



SIMULATIONS BASED LUMPED ELEMENT SOUND SOURCE MODEL

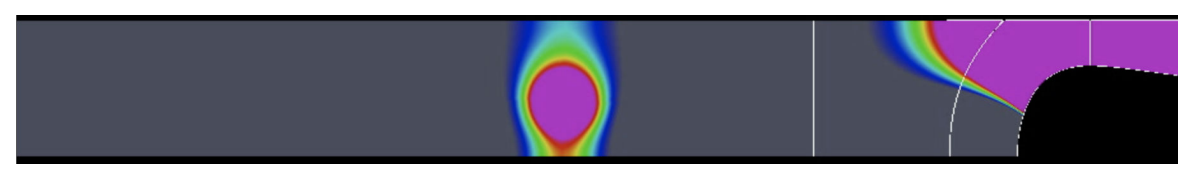

(a)

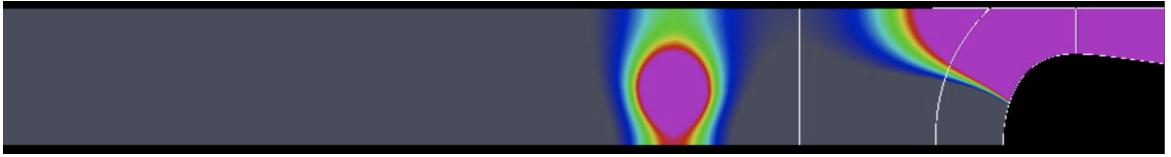

(b)

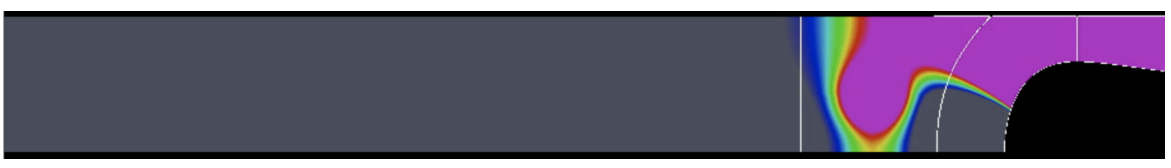

(c)

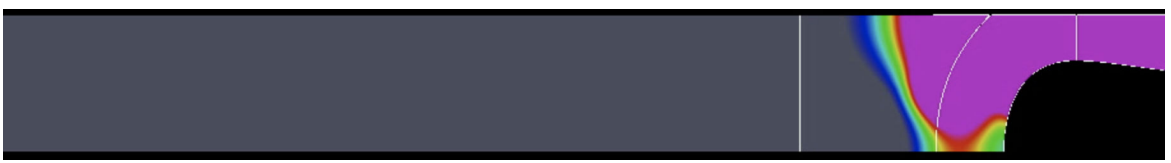

(d)

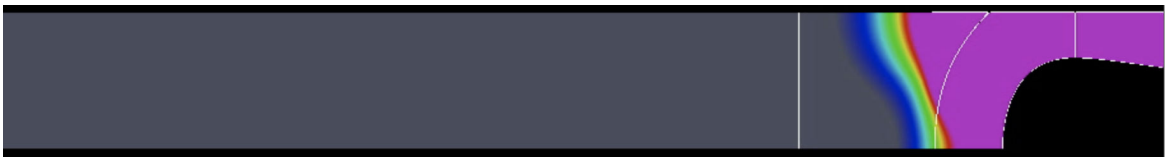

(e)

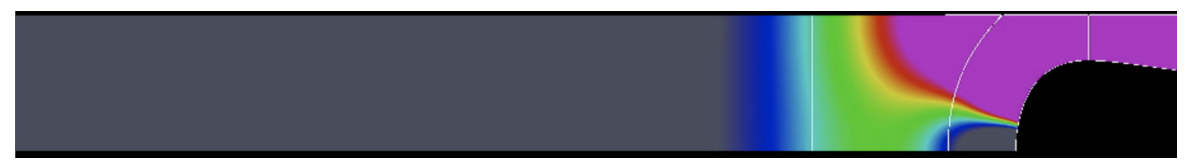

(f)

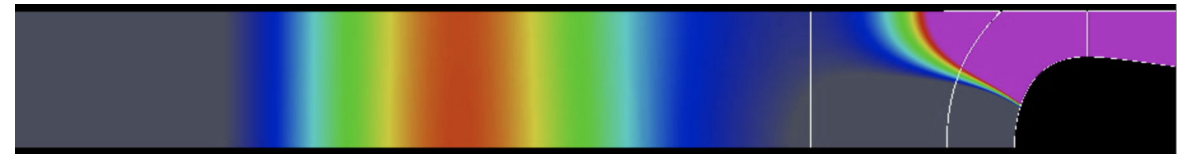

$(\mathrm{g})$

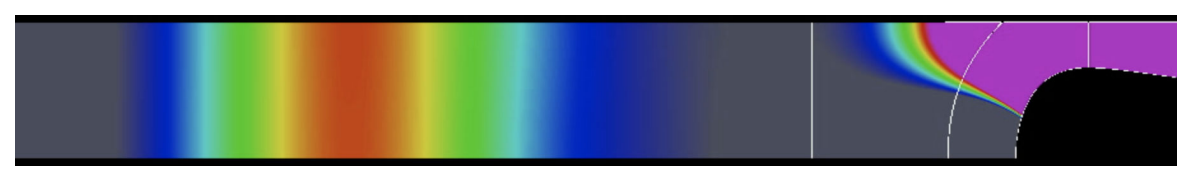

(h)

Figure 4.29: The formation of an upstream-traveling-plane acoustic wave due to compression of gas on the nozzle walls, shown in stages. 
Chapter 4 - Vortex-nozzle interaction: two-dimensional plane Euler SIMULATIONS BASED LUMPED ELEMENT SOUND SOURCE MODEL

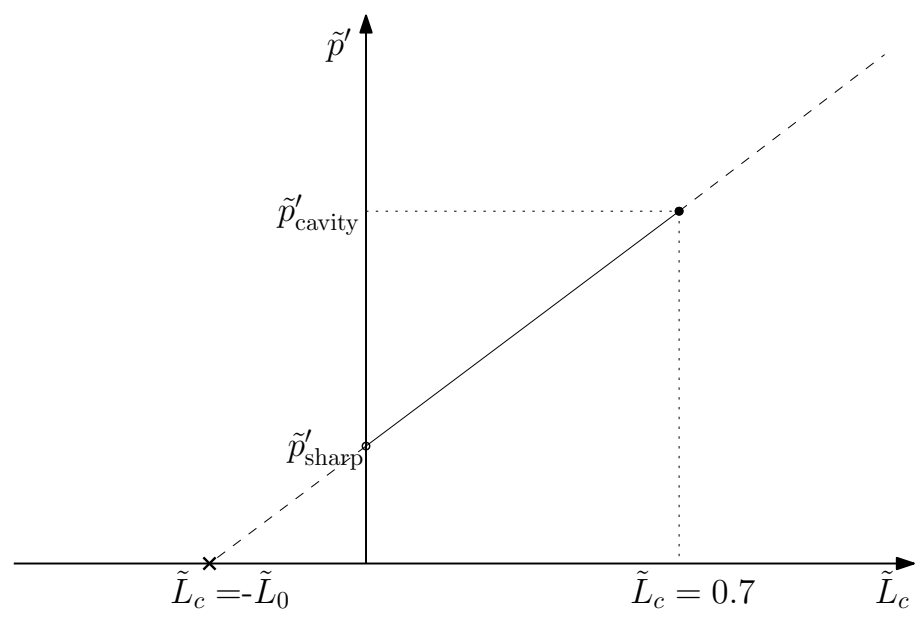

Figure 4.30: Sketch of extrapolation used for the estimation of $\tilde{L}_{0}$.

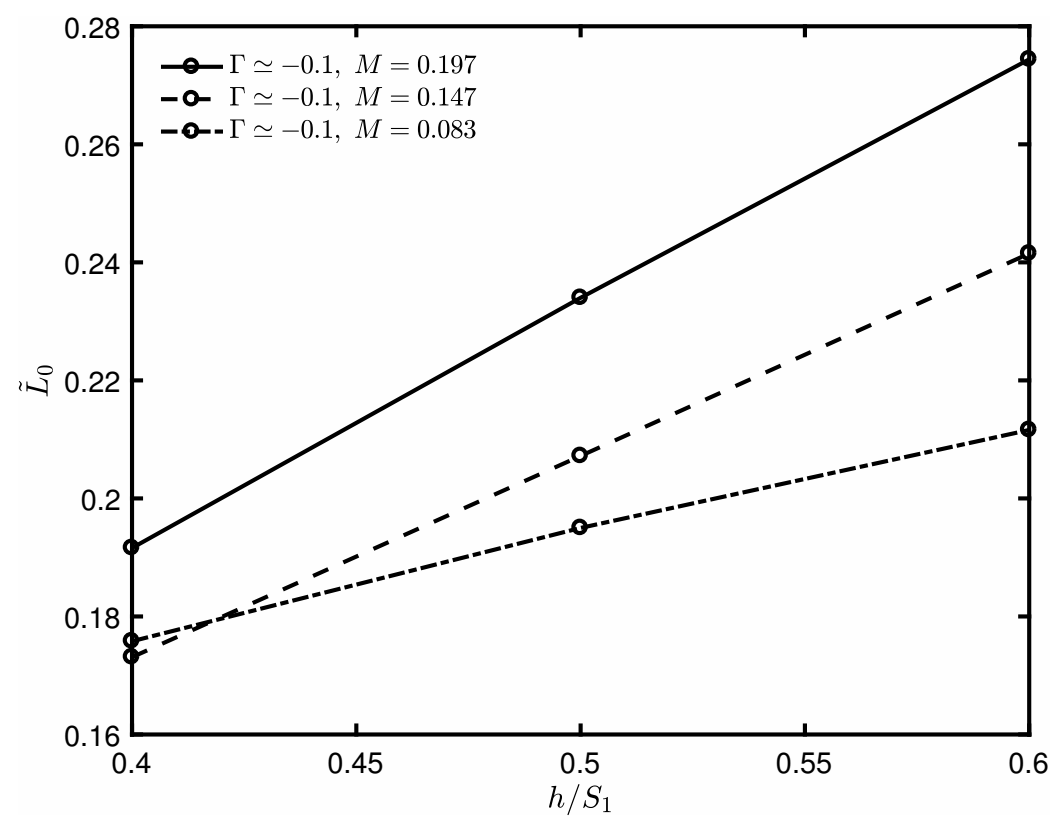

Figure 4.31: The residual length $\tilde{L}_{0}=L_{0} /\left(4 S_{1}^{3}\right)$ as a function of upstream release height $h / S_{1}$. Simulations for upstream Mach numbers $M=0.197,0.147,0.097,0.083$ were carried out. Form these results one can deduce that a taking $\tilde{L}_{0}=0.2$ is a fair approximation for the residual compressibility length $\tilde{L}_{0}$ in the range $0.05 \leq M \leq 0.2$. 
Chapter 4 - Vortex-nozzle interaction: two-dimensional plane Euler SIMULATIONS BASED LUMPED ELEMENT SOUND SOURCE MODEL

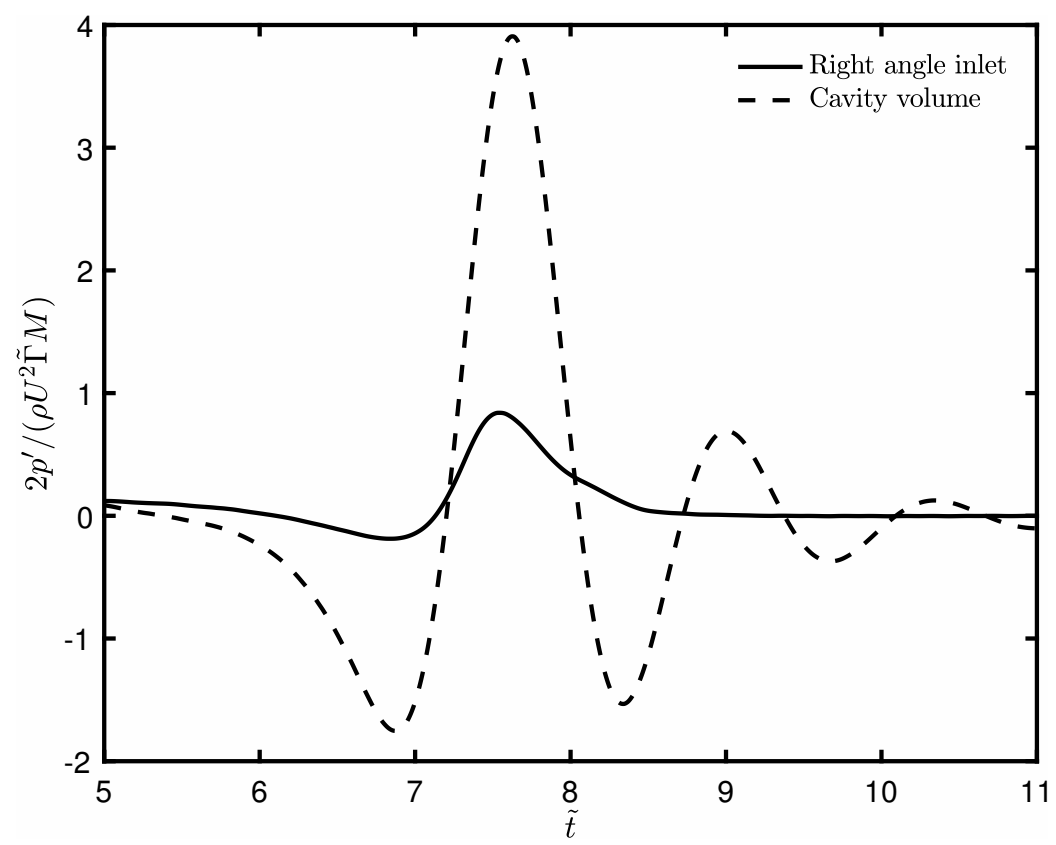

(a) Without $\tilde{L}_{\text {eff }}$ scaling

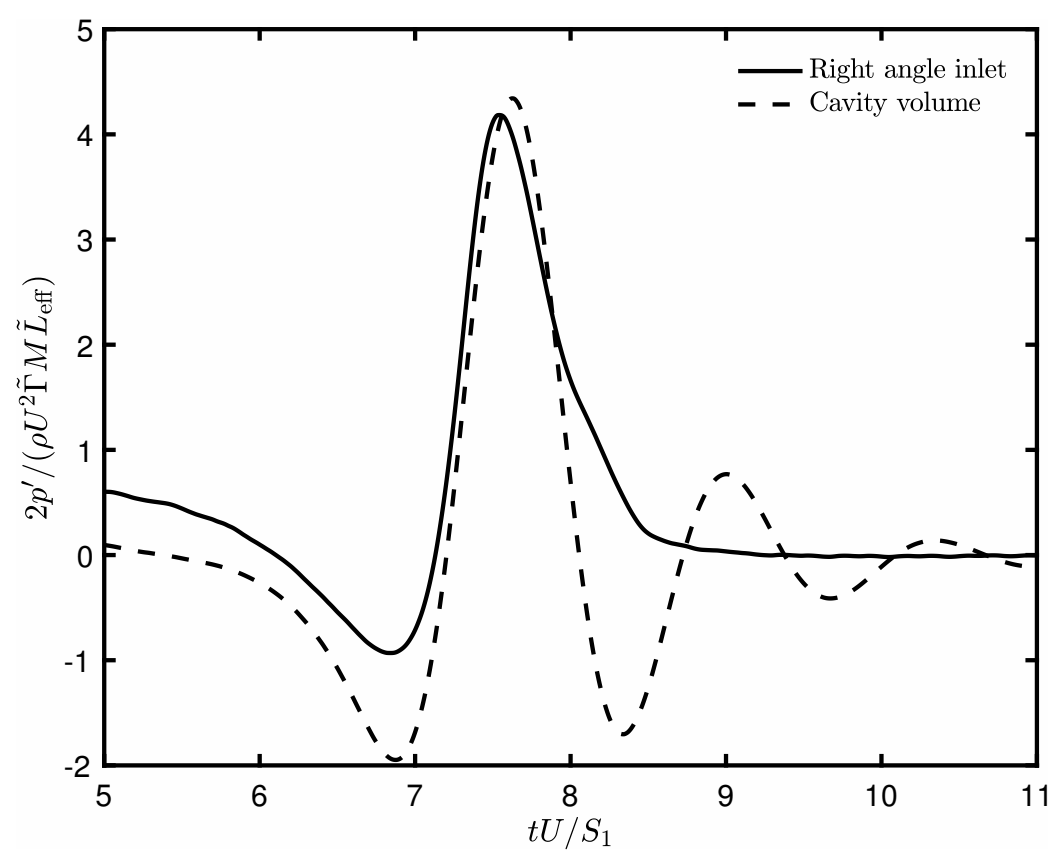

(b) With $\tilde{L}_{\text {eff scaling }}$

Figure 4.32: Influence of scaling with $\tilde{L}_{\mathrm{eff}}$. The acoustic response due to vortexnozzle interaction, obtained for $M=0.197, h=0.4 S_{1}$ and $\tilde{\Gamma} \simeq-0.1$ and the right angle inlet (solid line) and nozzle cavity (dashed line) geometries is shown. In subfigure (a) the signal is scaled by $\rho U^{2} \tilde{\Gamma} M$ and in subfigure (b) by $\rho U^{2} \tilde{\Gamma} M \tilde{L}_{\mathrm{eff}}$. One discerns a better collapse of the results when the effective length $\tilde{L}_{\mathrm{eff}}$ is taken into account in the scaling. 
Chapter 4 - Vortex-nozzle interaction: two-dimensional plane Euler SIMULATIONS BASED LUMPED ELEMENT SOUND SOURCE MODEL

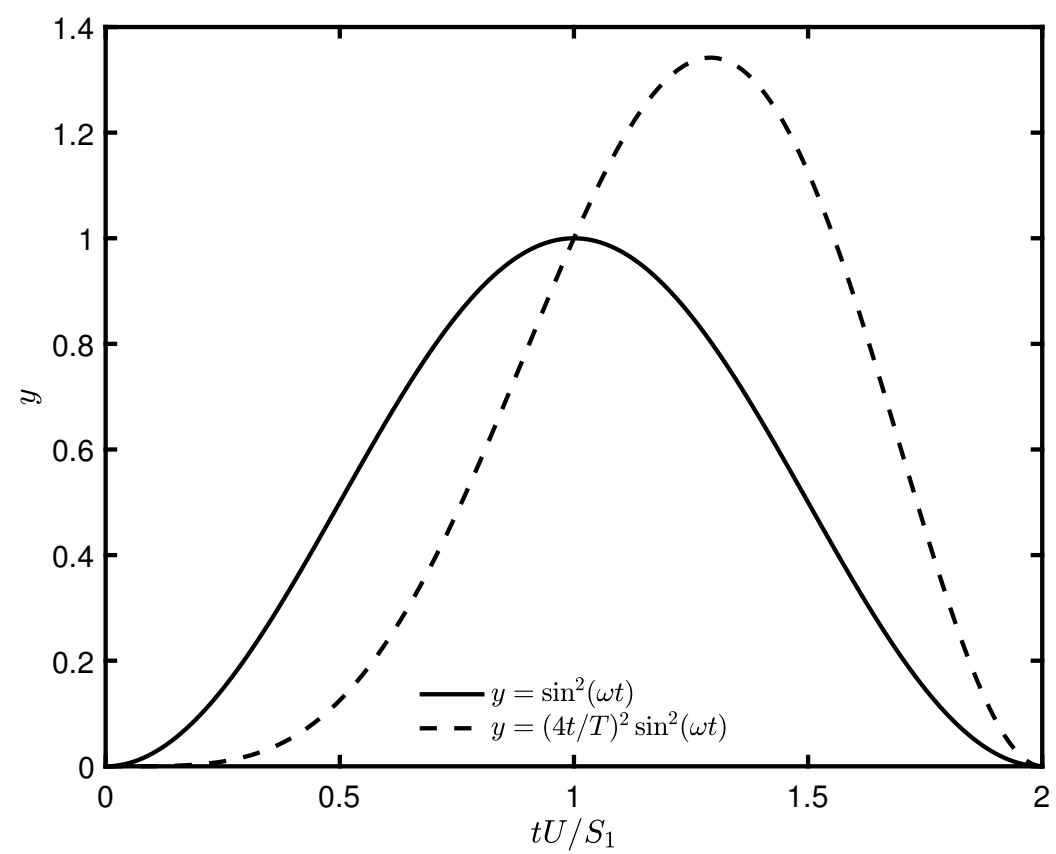

Figure 4.33: Sketch of $y=\sin ^{2}(\omega t)$ and $y=(4 t / T)^{2} \sin ^{2}(\omega t)$ functions on $0 \leq t \leq$ $T / 2$. Where $T=2 \pi / \omega=4 S_{1} / U$ is the period of oscillation, the this period is chosen such that $\omega T=4$. I.e. the first occurrence of a peak for $\sin ^{2}(\omega t)$ is when $t U / S_{1}=1$. Skewness of the pulse associated with the acceleration of the vortex is introduced by the factor $(4 t / T)^{2}$.

and

$$
\Delta B_{r}^{\prime}(t)=\left\{\begin{array}{l}
\beta \tilde{\Gamma}^{2} \frac{U^{2}}{2} \sin ^{2}(\omega(t-\tau)) \text { for } 0 \leq \omega(t-\tau) \leq 2 \\
0 \text { for all other } \omega(t-\tau)
\end{array}\right.
$$

The frequency is chosen $\omega=\pi U /\left(2 S_{1}\right)$, such that the dimensionless period of oscillation $U T / S_{1}=4$, as shown in Fig. 4.33. I.e. the first peak of $\sin ^{2}(\omega t)$ occurs for $t U / S_{1}=1$. The delay between the sources is assumed to be $\tau=S_{1} /(4 U)$.

The coefficients $\alpha$ and $\beta$ in Eq. (4.25) and (4.26) are determined by fit parameters. To take into account the slanted effect due to the acceleration of the vortex as it goes through the contraction, on the acoustic response signal obtained with the Euler model (e.g. Fig. 4.25) the parameters $\alpha$ and $\beta$ are made time dependent. To fit data obtained with both a sharp angle inlet nozzle and an integrated nozzle (with nozzle cavity) these parameters are chosen as follows 


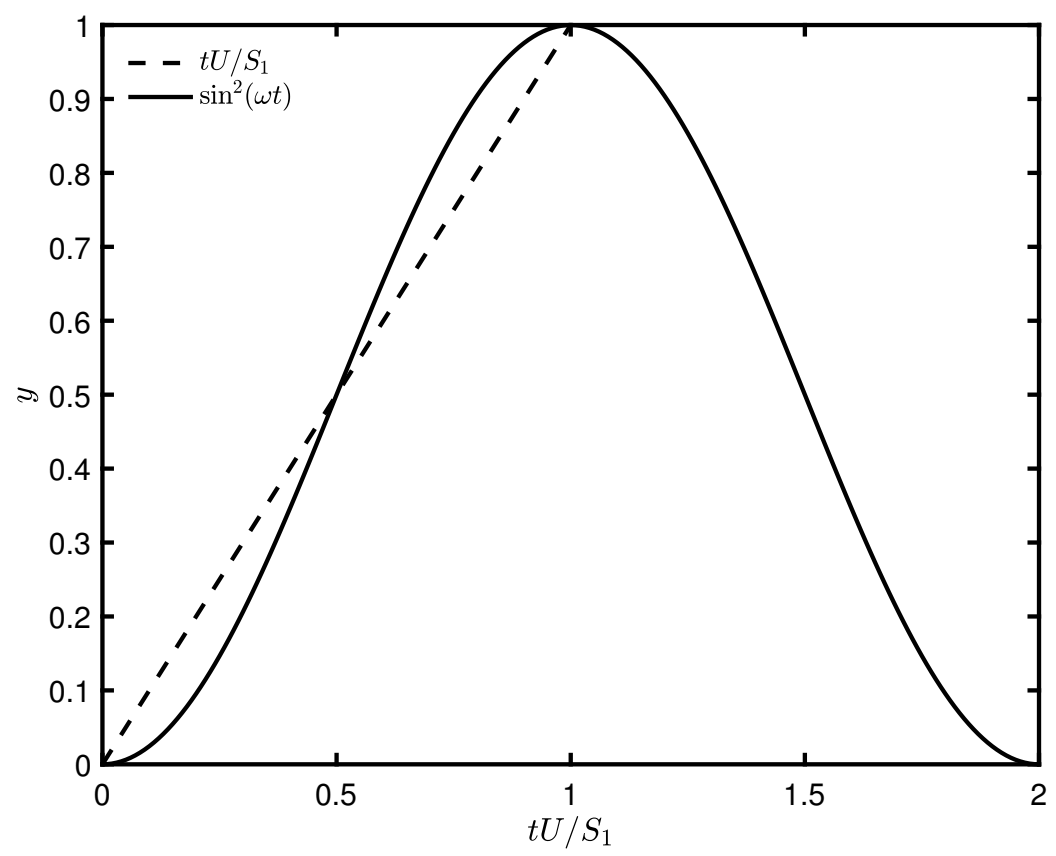

Figure 4.34: Sketch of first peak of $y=\sin ^{2}(\omega t)$ and $y=4 t / T$ on $0 \leq t \leq T / 2$. Where $T=2 \pi / \omega=4 S_{1} / U$ is the period of oscillation. The factor $4 t / T$ is shown as a dashed line. Note that for $t=T / 4$ the product $(4 t / T) \sin ^{2}(\omega t)$ equals one.

$$
\begin{aligned}
\alpha & =\kappa_{1}\left(\frac{4 t}{T}\right)^{l_{1}} \\
\beta & =\kappa_{2}\left(\frac{4 t}{T}\right)^{l_{2}}
\end{aligned}
$$

where $\kappa_{1}, \kappa_{2}, l_{1}$ and $l_{2}$ are fit parameters. The factor $4 t / T$ above is chosen such that when $\kappa_{1}=\kappa_{2}=l_{1}=l_{2}=1$ the product $(4 t / T) \sin ^{2}(\omega t)$ at $t=T / 4=1$ equals one, as shown in Fig. 4.34.

A single set of parameters $\kappa_{1}=1.5, \kappa_{2}=0.5, l_{1}=l_{2}=2$ has been used to fit Euler model results obtained with upstream Mach number $M=0.197$, vortexcore radius $R_{\Gamma}=0.3 S_{1}$ and release height $h=0.4 S_{1}$ for both the integrated nozzle and right angle inlet geometry. In Fig. 4.35 and 4.36 results of this fit are shown for $\tilde{\Gamma} \simeq-0.1$ and $\tilde{\Gamma} \simeq-0.9$ respectively. One observes that this is a fairly good agreement of the fit with the data. The peak values of the acoustic response are reproduced within $25 \%$. The shape of the initial dip in signal associated with the differentiation effect of the effective volume $V_{\text {eff }}$ is not exactly reproduced. This is most probably due to the fact that one should use different values for the skewness parameters $l_{1}$ and $l_{2}$. However, an optimization of the fit parameters has not been pursued, as the fit parameters used offer a qualitatively satisfactory description of the vortex-nozzle interaction sound source as 
Chapter 4 - Vortex-nOzzle interaction: two-dimensional Plane Euler SIMULATIONS BASED LUMPED ELEMENT SOUND SOURCE MODEL

well as a quantitative prediction within $25 \%$ of the acoustic response peak value.

In Fig. 4.37, the lumped model is compared to the Euler model for Mach numbers $M=0.197,0.097$ and 0.058 . The same set of fit parameters $\kappa_{1}=1.5$, $\kappa_{2}=0.5, l_{1}=l_{2}=2$ as above have been used for the lumped model. The results are compared for both the integrated nozzle and the right angle nozzle. The peak acoustic response values are reproduced within 30\%. One observes that the acoustic response peak of the lumped model fit for the right angle inlet geometry is somewhat narrower than for the Euler model results. Over all, however, the behavior is qualitatively reproduced.

\subsection{Response of a gradual ramp inlet nozzle}

In this section parameter studies of vortex-nozzle for the gradual ramp inlet nozzle geometry are presented.

First, the influence of the vortex-core radius $R_{\Gamma}$ was investigated. In Fig. 4.38 the scaled upstream acoustic response $2 p^{\prime} /\left(\rho U^{2} \tilde{\Gamma} M\right)$ due to vortex-nozzle interaction is shown as a function of dimensionless time $t U / S_{1}$ for vortex-core radii $R_{\Gamma}=0.2 S_{1}$ (unevenly dashed line), $R_{\Gamma}=0.3 S_{1}$ (solid line) and $R_{\Gamma}=0.4 S_{1}$ (dashed line). The results were obtained with fixed upstream Mach number $M=0.197$, vortex circulation $\tilde{\Gamma}=-0.860$ and upstream release height $h=0.5 S_{1}$. The same qualitative behavior is observed for the three vortex-core radii. There is a $13 \%$ deviation between the peak values of the acoustic response for $R_{\Gamma}=0.3 S_{1}$ and $R_{\Gamma}=0.2 S_{1}$. The deviation between the response for $R_{\Gamma}=0.3 S_{1}$ and $R_{\Gamma}=0.4 S_{1}$ is $14 \%$. One observes that the amplitude of the maximum peak value of the pulse has been reduced by a factor two compared to the right angle nozzle inlet geometry (Fig. 4.38). However, the pulse duration has been increased by a factor two. The time integral of the pulse seems to be constant. This behavior is consistent with what was predicted with the incompressible point vortex model (Hirschberg et al. 2017). Increasing the nozzle length would thus reduce the pulse height but the integral of the pulse would remain approximately constant. Note, however, that the present results are dominated by compressibility as a linear $\tilde{\Gamma}$ scaling prevails in the $\tilde{\Gamma}$ range considered.

When considering the coupling of the sound source with a longitudinal acoustic mode of the SRM with frequency $f \sim c /(2 L)$ (where $L$ is the length of the combustion chamber) the pulse width is not important as long as it remains short compared to $1 / f$. However, when the pulse length becomes comparable to or larger than $1 / f$, the net sound production will drastically be reduced by destructive interference. Hence in the absence of a nozzle cavity for 
100 Chapter 4 - Vortex-nozzle interaction: two-dimensional plane Euler SIMULATIONS BASED LUMPED ELEMENT SOUND SOURCE MODEL

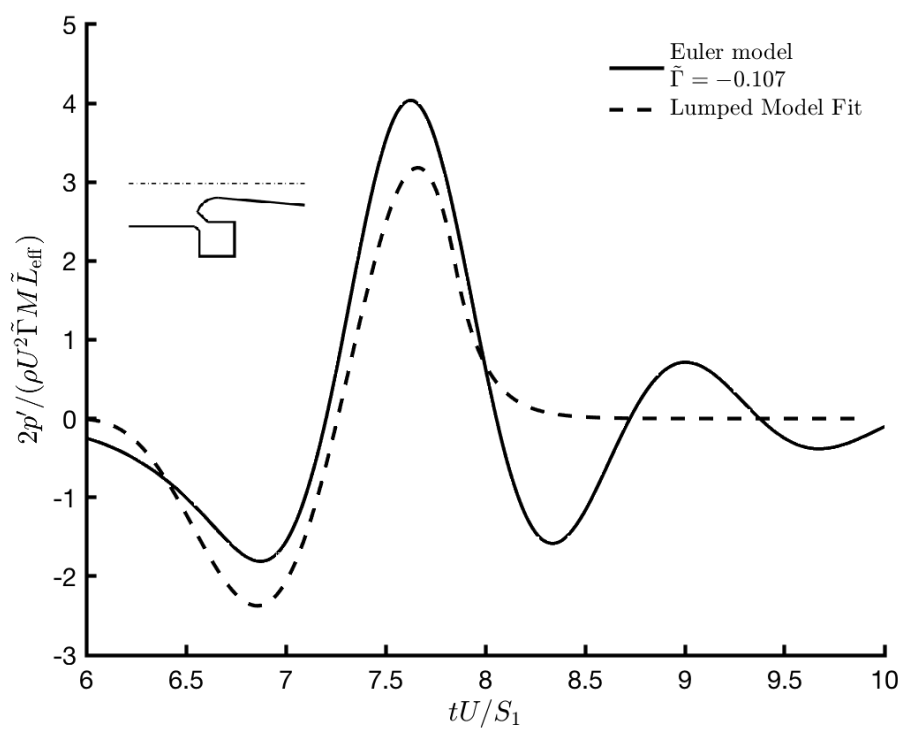

(a) Nozzle cavity $L_{\text {eff }}=0.9$

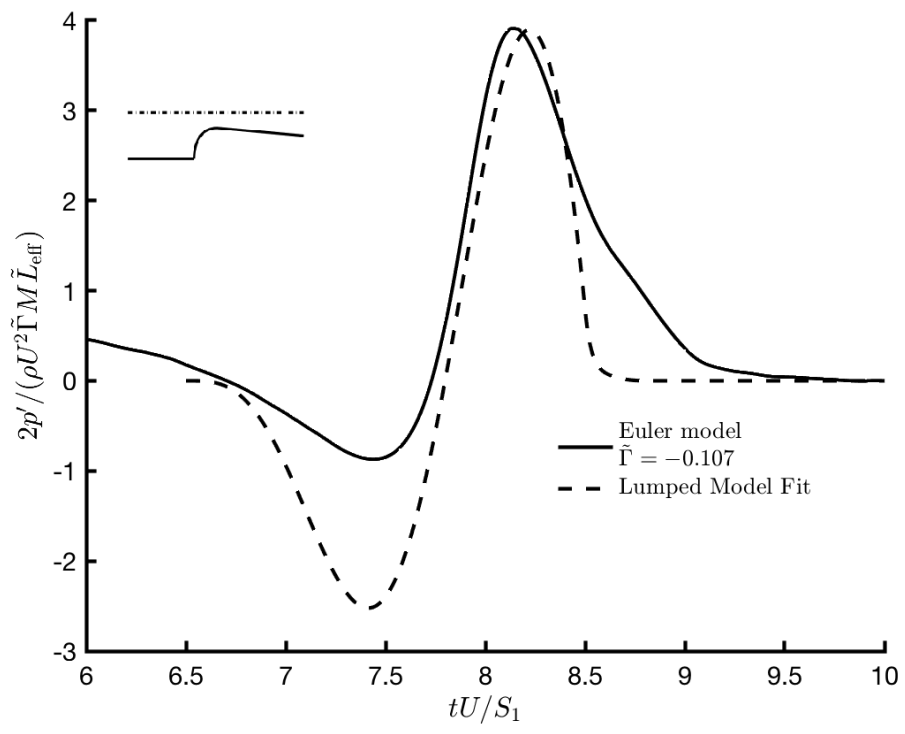

(b) Right angle inlet nozzle $L_{\text {eff }}=0.2$

Figure 4.35: Lumped model data fit with $\tilde{\Gamma}=-0.1, \omega=\pi U /\left(2 S_{1}\right), \alpha=1.5(2 \omega t / \pi)^{2}$ and $\beta=0.5(2 \omega t / \pi)^{2}$ thus a $t^{2} \sin ^{2}(\omega t)$ time dependence of pulses $\Delta B_{c}^{\prime}$ and $\Delta B_{r}^{\prime}$. 
Chapter 4 - Vortex-nozzle interaction: two-dimensional plane Euler 101 SIMULATIONS BASED LUMPED ELEMENT SOUND SOURCE MODEL

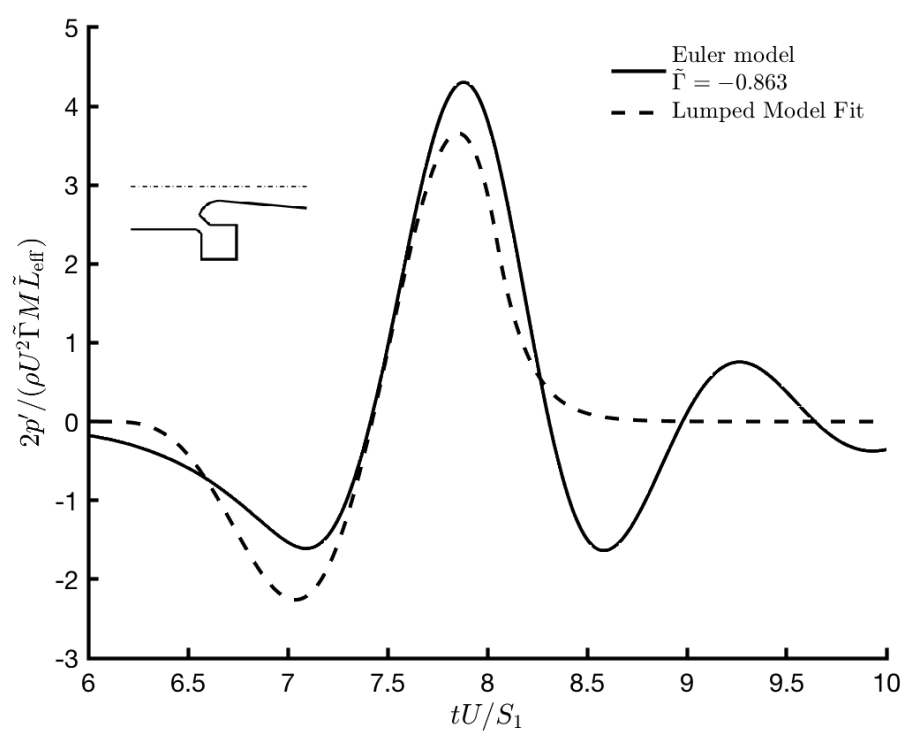

(a) Nozzle cavity volume $L_{\text {eff }}=0.9$

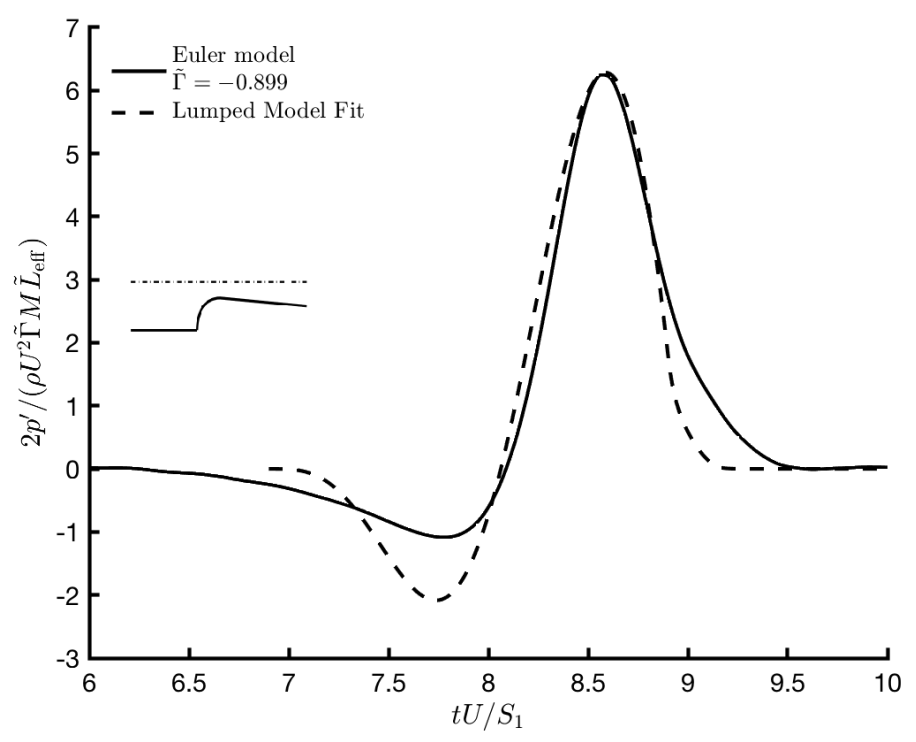

(b) Right angle inlet nozzle $L_{\text {eff }}=0.2$

Figure 4.36: Lumped model data fit with $\tilde{\Gamma}=-0.9, \omega=\pi U /\left(2 S_{1}\right), \alpha=1.5(2 \omega t / \pi)^{2}$ and $\beta=0.5(2 \omega t / \pi)^{2}$ thus a $t^{2} \sin ^{2}(\omega t)$ time dependence of pulses $\Delta B_{c}^{\prime}$ and $\Delta B_{r}^{\prime}$. 
102 Chapter 4 - Vortex-nozzle interaction: two-dimensional plane Euler SIMULATIONS BASED LUMPED ELEMENT SOUND SOURCE MODEL

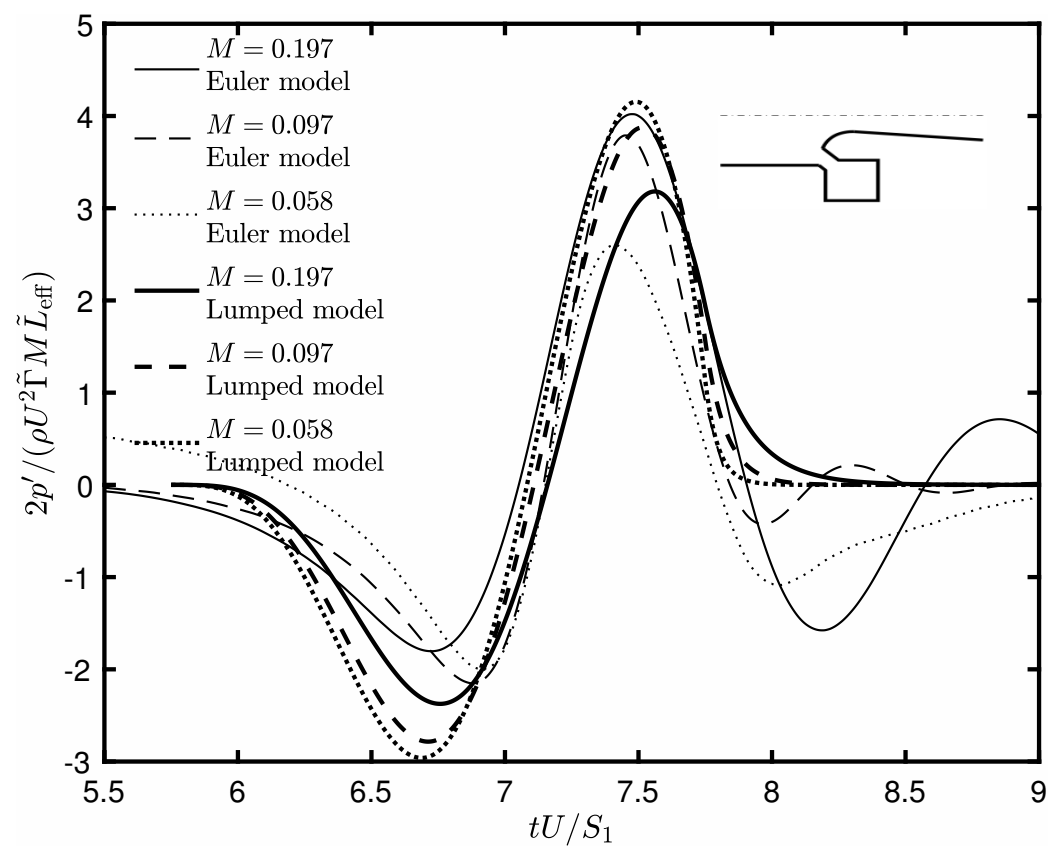

(a) Nozzle cavity $L_{\text {eff }}=0.9$

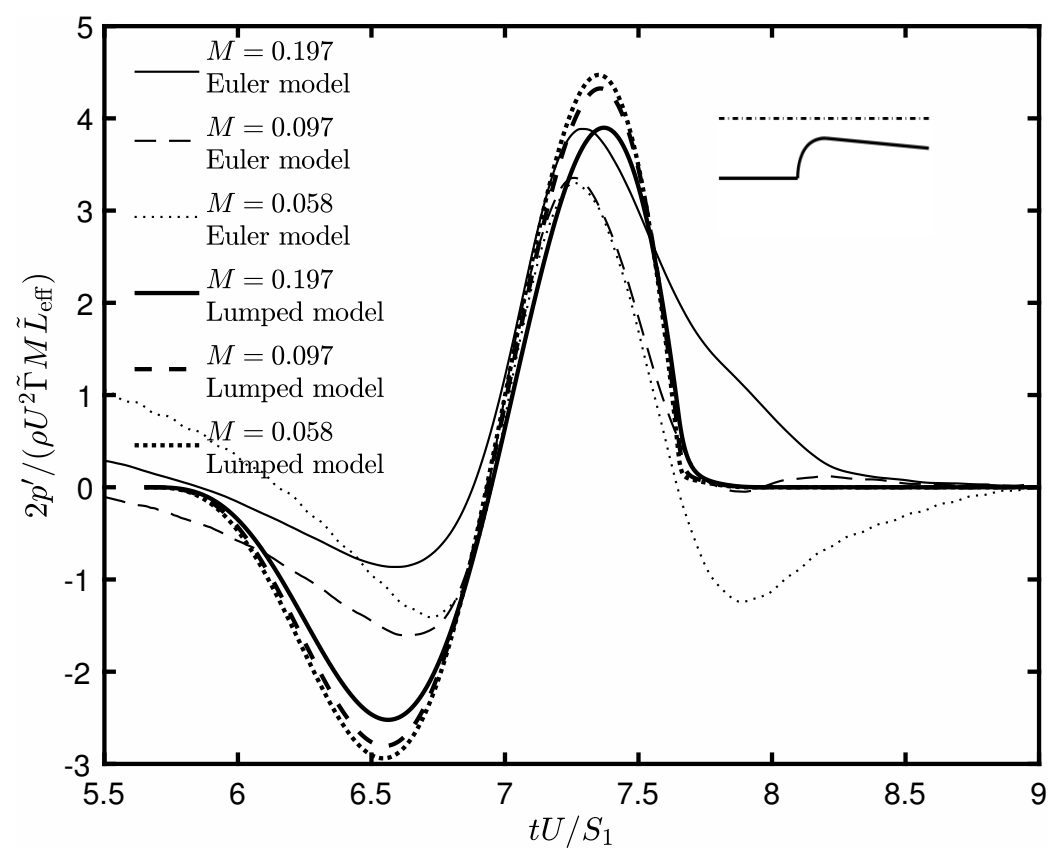

(b) Right angle inlet nozzle $L_{\text {eff }}=0.2$

Figure 4.37: Lumped model data fit varied Mach number $M$ with $\tilde{\Gamma}=-0.1, \omega=$ $\pi U /\left(2 S_{1}\right), \alpha=1.5(2 \omega t / \pi)^{2}$ and $\beta=0.5(2 \omega t / \pi)^{2}$ thus a $t^{2} \sin ^{2}(\omega t)$ time dependence of pulses $\Delta B_{c}^{\prime}$ and $\Delta B_{r}^{\prime}$. 
Chapter 4 - Vortex-nozzle interaction: two-dimensional plane Euler 103 SIMULATIONS BASED LUMPED ELEMENT SOUND SOURCE MODEL

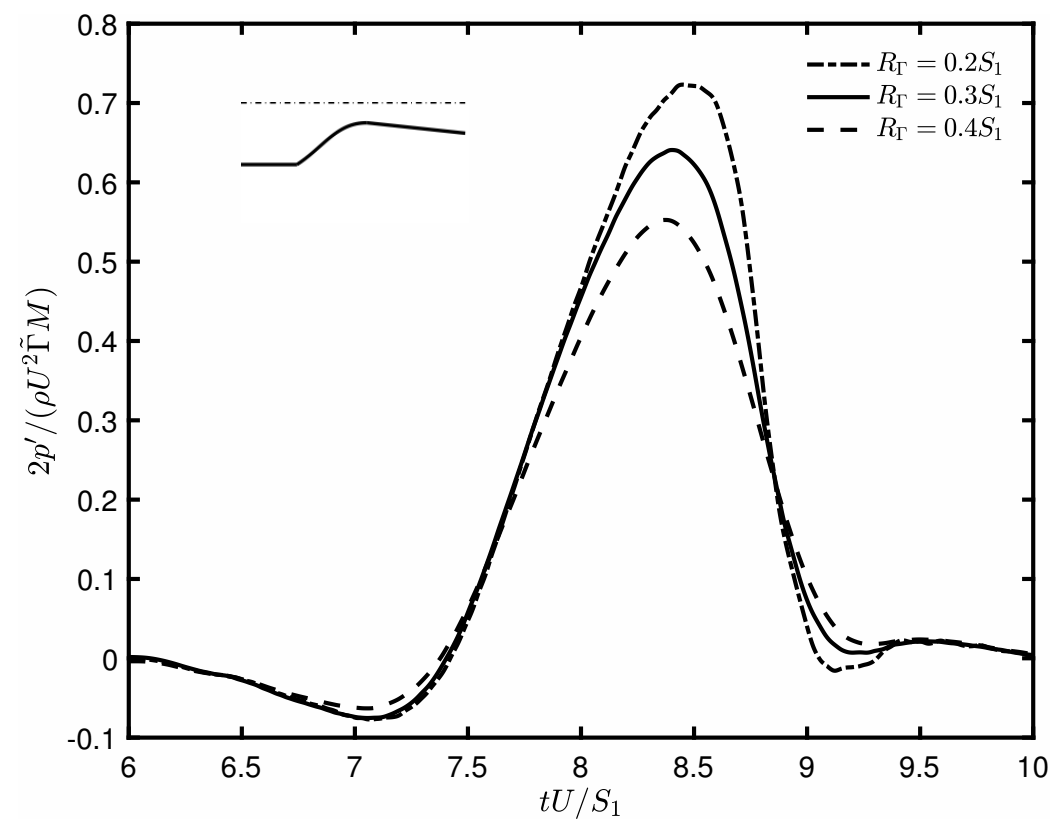

Figure 4.38: Influence of vortex-core radius $R_{\Gamma}$ for $\tilde{\Gamma} \simeq-0.9$. The scaled upstream acoustic response $2 p^{\prime} /\left(\rho U^{2} \tilde{\Gamma} M\right)$ due to vortex-nozzle interaction for varied vortex-core radius $R_{\Gamma}$ as a function of dimensionless time $t U / S_{1}$ is shown. Three vortex-core radii were used $R_{\Gamma}=0.2 S_{1}$ (unevenly dashed line), $R_{\Gamma}=0.3 S_{1}$ (solid line) and $R_{\Gamma}=0.4 S_{1}$ (dashed line). One observes that the upstream acoustic response is qualitatively the same for these three vortex-core radii. There is a $13 \%$ deviation between the peak values of the acoustic response for $R_{\Gamma}=0.3 S_{1}$ and $R_{\Gamma}=0.2 S_{1}$. The deviation between the response for $R_{\Gamma}=0.3 S_{1}$ and $R_{\Gamma}=0.4 S_{1}$ is $14 \%$.

$2 S_{1} f / U=S_{1} /(M L)>1$ the use of a gradual ramp might reduce the limit-cycle pulsation amplitude.

The influence of the upstream vortex release height $h$ on the scaled upstream acoustic response $2 p^{\prime} /\left(\rho U^{2} \tilde{\Gamma} M\right)$ due to vortex nozzle interaction as a function of dimensionless time $t U / S_{1}$ is shown in Fig. 4.39. The results shown have been obtained with dimensionless vortex circulation $\tilde{\Gamma}=-1.72$, vortex-core radius $R_{\Gamma}=0.3 S_{1}$ and upstream Mach number $M=0.197$. Three upstream release heights were used $h=0.4 S_{1}, 0.5 S_{1}$ and $0.6 S_{1}$. One observes that the upstream acoustic response is qualitatively the same for the three release heights. The deviation (14\%) between the peak values of the acoustic response for $h=0.4 S_{1}$ and $h=0.5 S_{1}$ is the same as between $h=0.4 S_{1}$ and $h=0.6 S_{1}$. One concludes that overall the upstream acoustic response due to vortex-nozzle interaction is not strongly influenced by $h$ for $0.4 \leq h / S_{1} \leq 0.6$.

To find the circulation for which a quadratic scaling in dimensionless vortex circulation dominates, simulations with unrealistically large values of $|\tilde{\Gamma}|$ have 
104 Chapter 4 - Vortex-nozzle interaction: two-dimensional plane Euler SIMULATIONS BASED LUMPED ELEMENT SOUND SOURCE MODEL

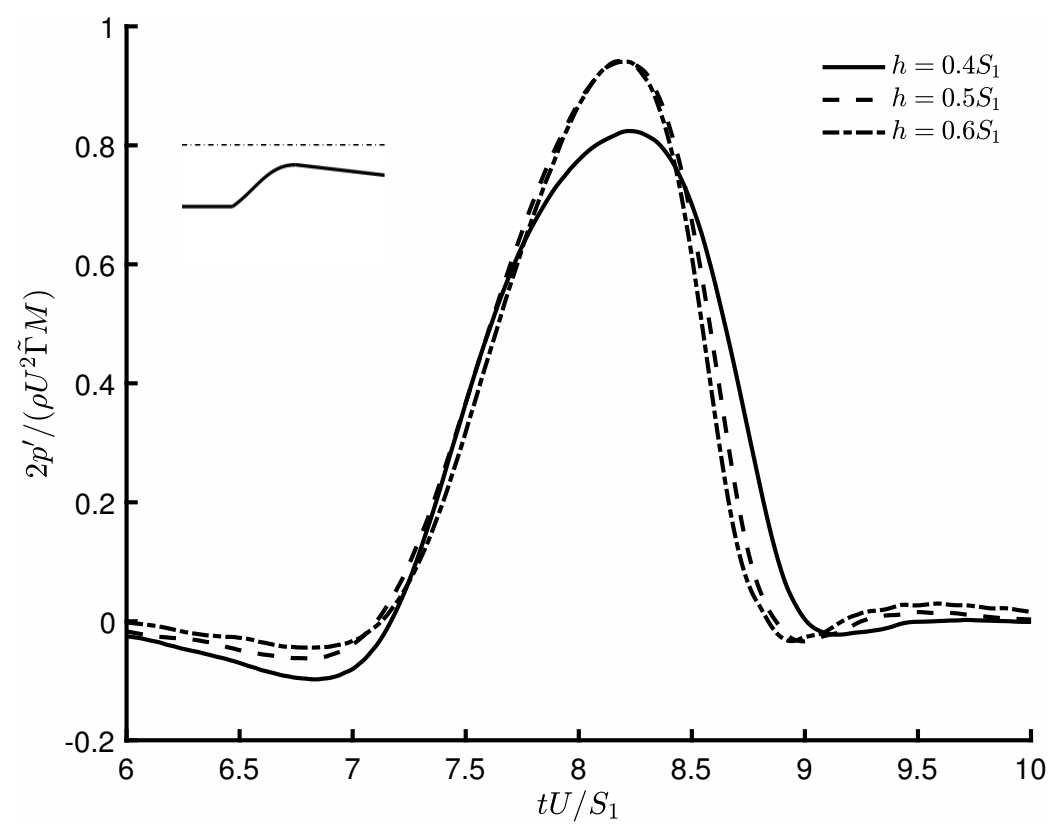

Figure 4.39: Influence of vortex release height $h$ for $\tilde{\Gamma} \simeq-1.7$. The scaled upstream acoustic response $2 p^{\prime} /\left(\rho U^{2} \tilde{\Gamma} M\right)$ due to vortex-nozzle interaction, as a function of dimensionless time $t U / S_{1}$. The influence of the upstream vortex release height $h$ was investigated for the gradual ramp inlet nozzle geometry. Three upstream release heights were used $h / S_{1}=0.4,0.5$ and 0.6. One observes that the upstream acoustic response is qualitatively the same for these three release heights. One concludes that overall the upstream acoustic response due to vortex-nozzle interaction is not influenced significantly for $0.4 \leq h / S_{1} \leq 0.6$. 
Chapter 4 - Vortex-nOzzle interaction: two-dimensional Plane Euler 105 SIMULATIONS BASED LUMPED ELEMENT SOUND SOURCE MODEL

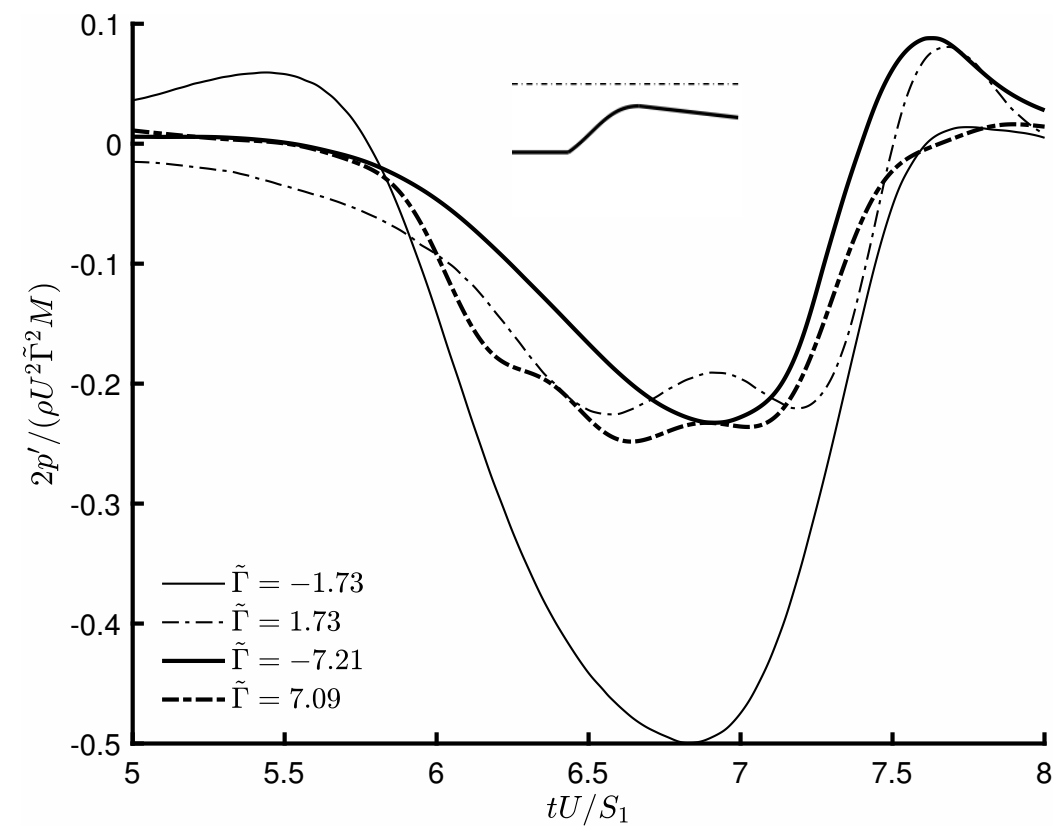

Figure 4.40: Transition form linear to quadratic scaling in vortex circulation $\tilde{\Gamma}$. The scaled upstream acoustic response $2 p^{\prime} /\left(\rho U^{2} \tilde{\Gamma}^{2} M\right)$ due to vortex-nozzle interaction as a function of dimensionless time $t U / S_{1}$ is shown. The transition to scaling quadratic in $\tilde{\Gamma}$ is demonstrated. Thin lines are for $\tilde{\Gamma}= \pm 1.73$, thick lines are for $\tilde{\Gamma}=-7.21$ (solid thick line) and $\tilde{\Gamma}=7.09$ (unevenly dashed thick line). One observes that the scaling behavior quadratic in $\Gamma$ only for unrealistically high $|\tilde{\Gamma}| \simeq=7$.

been carried out. In Fig. 4.40, the dimensionless upstream acoustic response $2 p^{\prime} /\left(\rho U^{2} \tilde{\Gamma}^{2} M\right)$ as a function of dimensionless time $t U / S_{1}$ is shown for $|\tilde{\Gamma}|=1.7$ and $|\tilde{\Gamma}| \simeq 7$. The results were obtained with a fixed upstream Mach number $M=0.197$, vortex release height $h=0.4 S_{1}$ and vortex-core radius $R_{\Gamma}=0.3 S_{1}$. The thin lines indicate $\tilde{\Gamma}= \pm 1.73$, thick line $\tilde{\Gamma}=-7.21$ and unevenly dashed line $\tilde{\Gamma}=7.09$. The transition to a scaling quadratic in vortex circulation $\tilde{\Gamma}$ is observed for $|\tilde{\Gamma}| \simeq 7$. This scaling behavior is quadratic in $\Gamma$ only for very high (unrealistic) absolute values of vortex circulation. For $\tilde{\Gamma}= \pm 1.7$ the linear scaling is still valid.

\subsection{Conclusion}

To investigate the production of sound due to the interaction of a vortex with a choked nozzle, a lumped element model has been developed. This model can be used for parametric studies of self-sustained pressure oscillations in the combustion chamber of a SRM. Two fundamental sound production mechanisms have been identified using the vortex sound theory (section 4.4):

- sound production due to the ingestion of the vortex by the nozzle, 
106 Chapter 4 - Vortex-nozzle interaction: two-dimensional plane Euler SIMULATIONS BASED LUMPED ELEMENT SOUND SOURCE MODEL

- sound produced due to compression of the gas around the nozzle inlet. Both sound sources can be represented by a dipole sound source, which in a plane wave lumped element model is a time dependent discontinuity in the total enthalpy $B$. These sources are referred to as $\Delta B_{r}^{\prime}$ (the nozzle radiation source) and $\Delta B_{c}^{\prime}$ (compression source). While both sources scale with $U^{2} / 2, \Delta B_{c}^{\prime}$ is proportional to $\tilde{\Gamma}$ and $\Delta B_{r}^{\prime}$ to $\tilde{\Gamma}^{2}$, where $\tilde{\Gamma}=\Gamma /\left(U S_{1}\right)$ is the dimensionless vortex circulation (Eq. (4.12) and (4.13)).

Using a two-dimensional Euler model (EIA) a systematic study of the influence of various parameters has been carried out for the radiation of sound in a semi-infinite channel terminated by a choked nozzle. Two nozzle geometries typically found in the literature for SRMs have been studied in detail: a nozzle with surrounding cavity of volume $V_{c}$ (Fig. 4.2 (a)) and a nozzle with an inlet that forms a right angle with the upstream channel (Fig. 4.2 (b)).

For these configurations the sound source $\Delta B_{r}^{\prime}$ radiates an inverse of the pulse $\Delta B_{r}^{\prime}$ multiplied by a factor $(\gamma+1) M / 2$, where $M$ is the nozzle inlet Mach number (Fig. 4.7). The sound source $\Delta B_{c}^{\prime}$ radiates as the time derivative $\frac{d \Delta B_{c}^{\prime}}{d t}$ of the inverse of the pulse $\Delta B_{c}^{\prime}$ multiplied by a factor $L_{\text {eff }} / c$, where $L_{\text {eff }}$ is an effective length and $c$ is the speed of sound at the nozzle inlet (Eq. (4.14)). The effective length $L_{\text {eff }}$ is the ratio $V_{\text {eff }} / S_{p}$ of the effective volume $V_{\text {eff }}=V_{c}+V_{0}$ with $V_{c}$ the nozzle cavity volume, $V_{0}$ the residual compressibility volume and the combustion chamber cross-sectional area $S_{p}$ (section 4.9). The corresponding amplitude scales with the upstream Mach number $M$ as well.

The added volume $V_{0}$ takes the effect of gas compression outside the cavity into account. The numerical simulations indicate that $V_{0}$ is to a small degree dependent on of upstream vortex release height $h$ (Fig. 4.31). This seems at least intuitively plausible as there is a larger amount of gas between the vortex and the contraction wall for vortices released at larger distance $h$ from the lower channel wall. The effect of the Mach number $M$ on $V_{0}$ is minor.

For dimensionless vortex circulations $|\tilde{\Gamma}| \leq 0.5$ the contribution of $\Delta B_{r}^{\prime}$ is negligible. For $|\tilde{\Gamma}|=\mathcal{O}(1)$ in absence of cavity $\left(V_{c}=0\right)$ both sound sources contribute equally to the radiative sound pulse (Fig. 4.26). Simple time dependencies have been proposed for both $\Delta B_{r}^{\prime}$ and $\Delta B_{c}^{\prime}$, which result in fair agreement with the Euler model in the relevant parameter range $0.05 \leq M \leq 0.2$ and $-1 \leq \tilde{\Gamma} \leq-0.1$. In this parameter range the total enthalpy acoustic pulse scales with $\frac{U^{2}}{2} \tilde{\Gamma} M \tilde{L}_{\text {eff }}$.

The lumped element source model could be implemented in an acoustical model of the SRM (e.g. the harmonic oscillator model described in chapter 2) to predict limit-cycle oscillation amplitude as has been done for the incompressible flow model (Hirschberg et al. 2018). Furthermore, the procedure followed here 
Chapter 4 - Vortex-nOzzle interaction: two-dimensional Plane Euler 107 SIMULATIONS BASED LUMPED ELEMENT SOUND SOURCE MODEL

can be used to obtain a lumped element sound source model from other numerical simulations of e.g. axi-symmetrical flows.

Smoothing the inlet geometry (section 4.11) appears to reduce the radiation pulse amplitude by a factor two compared to amplitudes generated with a right angle inlet nozzle. However, the pulse duration increases by a factor two, implying that the time integral $\int B^{\prime} d t$ of the pulse is approximately unchanged (Fig. 4.38 and 4.39). If the duration of the pulse remains short compared to the longitudinal frequency $f \simeq c /(2 L)$ of the combustion chamber, there would then be no befit in using such a long nozzle inlet for the reduction of the limitcycle pulsation amplitude. If the sound pulse length is comparable or larger than $f$, a reduced limit-cycle amplitude is expected. It is furthermore striking that compressibility effects are still quite important for the gradual ramp inlet configuration. This is concluded from the fact that scaling with the square of the dimensionless vortex circulation $\tilde{\Gamma}^{2}$ is only approached for unrealistically large vortex circulations $|\tilde{\Gamma}|>2$ (Fig. 4.40). This implies that $G_{c}$ is the dominant part of the Green's function, $G=G_{r}+G_{c}$.

In any case the presence of a nozzle-cavity volume should be avoided, when low limit-cycle pulsation amplitudes are sought. 



\title{
Chapter 5
}

\section{Analytical energy balance prediction of limit-cycle amplitude}

\begin{abstract}
Cold gas scale model experiments $(1 / 30)$ demonstrate that coupling of vortex shedding with acoustic standing waves can produce pressure oscillations of the same level as observed in large solid rocket motors. An analytical acoustical energy balance model is proposed, in which the system is described as a single mode acoustic resonator and the pulsations are assumed to be purely harmonic. The selected acoustic mode number is an input to the model. Quasi-steady linear models are used to describe losses of acoustic energy by vortex shedding at a thermal inhibitor ring, radiation at the nozzle and friction within the porous injection wall used for gas injection. Sound production is predicted by using a 2-D planar point vortex model combined with vortex sound theory. The model demonstrates that the sound production due to interaction of the vortex with the cavity surrounding the integrated nozzle is dominant, explaining previous results of cold gas and hot-gas scale models. The effect of vortex ingestion by the nozzle is negligible. Aspects of the nozzle geometry, other than the cavity volume, are not critical. The model predicts pressure pulsations within a factor 2 , when the circulation of the vortices is taken one third of the maximum available circulation. This reduction factor of the circulation is assumed to be a consequence of turbulence. The Mach number corresponding to the maximum of pulsation is predicted within $20 \%$ in a range comparable to results obtained by axis-symmetrical numerical flow simulations.
\end{abstract}

This chapter is based on a published article: L. Hirschberg, T. Schuller, J. Collinet, C. Schram, A. Hirschberg, "Analytical model for the prediction of pulsations in a cold-gas scale-model of a solid rocket motor", Journal of Sound and Vibration, vol. 419 (2018) 


\subsection{Introduction}

Solid rocket motors (SRMs) can display strong acoustic oscillations (Culick 2004; Anthoine 2014; Fabignon et al. 2016). For small engines these are often driven by a coupling between the combustion and acoustic standing waves in the engine (Greatrix 2011; Flandro et al. 2007). In large SRMs the oscillations can be sustained by coupling between vortex shedding and acoustic standing waves (Vuillot 1995; Vuillot and Casalis 2004). A modulation of the combustion rate by the acoustic field may also alter these pulsations, but is not absolutely necessary to sustain pulsations.

Detailed numerical simulations (CFD) of these sustained pressure oscillations in SRMs still remain challenging and were recently reviewed in (Fabignon et al. 2016). This makes systematic parametric CFD studies of this phenomenon in SRMs wildly impractical. Thus, there is a need for simplified models to accompany these numerical investigations. These could eventually be used for interpolation or extrapolation of experimental or numerical results.

Most of the past analytical research effort focuses on exponential growth of instabilities (Culick 2006; Flandro 2003; Fischbach and Majdalani 2009). This has been developed using linear theory, and as such is not capable of predicting limit-cycle amplitudes. Furthermore, most existing theories were developed for combustion related instabilities, with limited progress having been made to date for cases instabilities triggered by hydrodynamic interactions (Culick 2006).

Originally (Brown et al. 1981) it was believed that vortex shedding could only occur at thermal inhibitor rings between segments of the propellant. This has been referred to as obstacle vortex shedding (OVS). However, Vuillot (Vuillot 1995) and Dotson et al. (Dotson et al. 1997) have shown that vortices can be formed in the absence of inhibitors. This is due to the intrinsic instability of the so-called Taylor flow generated by the combustion (Casalis and Vuillot 2004; Laboureur et al. 2010) and is referred to as surface vortex shedding (SVS). Vortex shedding can also occur at the edge of an abrupt expansion in the combustion chamber cross-section. This is called Angle Vortex Shedding (AVS) and is similar to OVS. In this chapter, OVS is focused on as a mechanism for vortex shedding and a model is developed to understand how OVS vortices interact with the sound field within a SRM.

Experimental results (Anthoine 2000) display oscillation bursts in specific Mach number ranges corresponding to a particular acoustic mode and a hydrodynamic mode. At the pulsation maximum, the oscillation frequency coincides with the acoustic mode eigenfrequency. Within such a burst the oscillation frequency increases with increasing Mach number as observed in actual SRMs 
(Dotson et al. 1997; Fabignon et al. 2003; Kuentzmann et al. 2004). A famous example of such data is shown in Fig. 5.1.

Fig. 5.1 is a reproduction of the high amplitude pulsations detected in the Titan 4 SRM. One observes bursts of pulsations around the eigenfrequency of the first acoustic mode, corresponding to a standing wave of approximately one half wavelength along the combustion chamber. The Mach number at the nozzle inlet decreases with time due to combustion while the choked nozzle throat diameter remains almost constant. Within one burst, the frequency decreases as a function of time, hence it increases with increasing Mach number. This frequency signature is a consequence of the time delay in the convection of vortices between the vortex shedding position in the combustion chamber and the nozzle, where sound is generated. Data such as shown in Fig. 5.1 have motivated investigations of OVS as a strong source of instabilities (Anthoine 2000; Anthoine et al. 2002; Anthoine et al. 2003; Fabignon et al. 2003; Kuentzmann et al. 2004) in SRMs.

Anthoine et al. (Anthoine 2000; Anthoine et al. 2002; Anthoine et al. 2003) confirmed the importance of vortex-nozzle interaction in sound production. In the cold gas experiments (Anthoine 2000) the combustion is replaced by injection of air through a porous wall to reproduce the flow at a 1/30 scale in a Ariane 5 SRM. Anthoine (Anthoine 2000) found ratios $p_{\text {rms }} / p$ of the root-meansquare (rms) acoustic pulsation amplitude $p_{\text {rms }}$ to static pressure $p$ of the order of $10^{-3}$. This corresponds to the amplitudes observed in full scale firing test (Fabignon et al. 2003) and scale models with combustion (Fabignon et al. 2003; Gallier et al. 2009). Anthoine observed the characteristic frequency signatures observed in SRMs (Dotson et al. 1997; Fabignon et al. 2003; Kuentzmann et al. 2004), i.e. an increasing frequency around the acoustic mode frequency with increasing Mach number around each maximum of pulsation amplitude. This confirms that these signatures can be accounted for by convective delay of vortices.

Anthoine et al. (Anthoine 2000; Anthoine et al. 2002) proposed an analytical model to estimate the pulsation amplitude by balancing the vortex sound power to radiation losses at the nozzle. In the present chapter an improved analytical model is provided based on vortex sound theory in which the losses are estimated using quasi-steady linear models. This includes radiation at the nozzle, the effect of the vortex shedding at the inhibitor (Durrieu et al. 2001) and the sound absorption by the porous wall (Schmidt 2015). The effect of convection in the nozzle radiation model, ignored by Anthoine (Anthoine 2000), is also included using the expressions from Marble and Candel (Marble and Candel 1977) and Culick (Culick 2006).

The objective of this chapter is to develop an analytical nonlinear energy bal- 


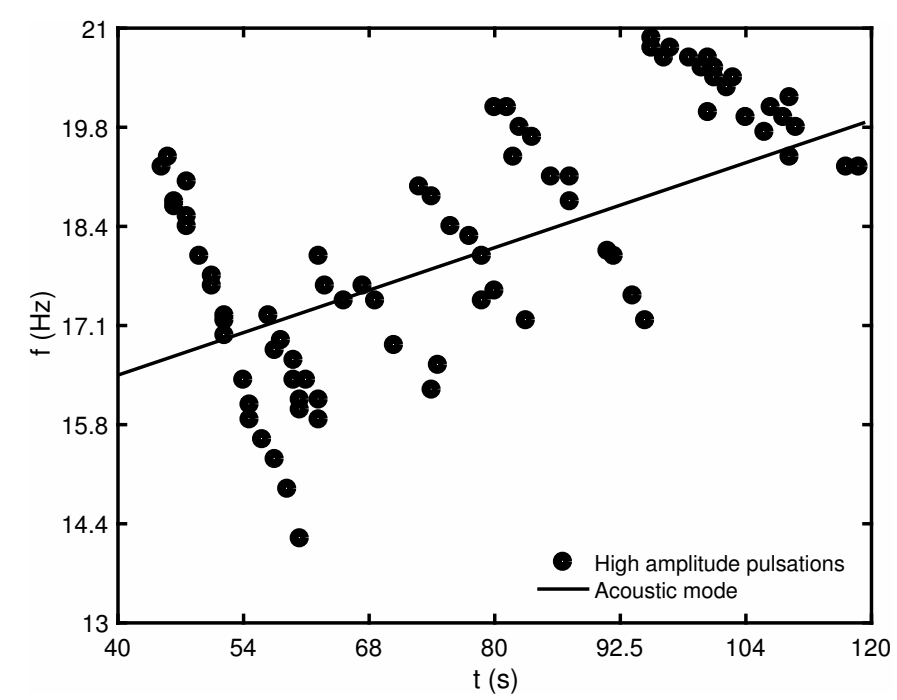

Figure 5.1: Time dependence of the frequency of pulsations observed in a SRM. Titan 4 data, figure after figure 4 in (Dotson et al. 1997). One observes six sets of measurements corresponding to bursts of high amplitude pulsation. Within each burst the frequency of oscillation decreases with increasing time, corresponding to an increase of oscillation frequency with increasing Mach number.

ance model that can be used to simulate limit cycles in SRMs focusing on sources of sound due to vortex dynamics only. Another aim is to use the model for parametric investigations of effects due to changes of the nozzle geometry. As a first validation step the proposed model is compared to cold-gas scale model experimental data (Anthoine 2000; Anthoine and Lema 2009).

The model considered is a nonlinear energy balance, in which saturation of the amplitude is assumed to be reached as a result of the formation of discrete vortical structures. The circulation and path of these vortical structures is assumed to be independent of the acoustical pulsation amplitude, because the acoustically induced velocity perturbations are typically two orders of magnitude smaller than the main flow velocity. Hirschberg et al. (Hirschberg et al. 2016) developed a model using the same nonlinear energy balance but used a crude vortex-dynamics model, with what amounts to three fit parameters. The model presented in this paper includes an improved vortex dynamics description that reduces the number of fit parameters to one. It allows for parametric studies of the influence of the shape of the nozzle inlet which is not possible using the model in (Hirschberg et al. 2016). Moreover, the present model takes into account both the contributions of the nozzle cavity volume, $V_{c}$, and the ingestion of the vortex by the nozzle as sources of sound. This allows for an analytical investigation of the contribution of these effects. Such an investigation that has not been carried out previously. 
The nonlinear energy balance approach does not allow for the variation of the oscillation frequency. That being the case, the lumped element modeling approach is used to develop an equation used to estimate $(M / f)(\mathrm{d} f / \mathrm{d} M)$. The quantity $(M / f)(\mathrm{d} f / \mathrm{d} M)$ is used to verify if the use of linear loss models is satisfactory.

Section 5.2 describes the cold gas experiments of Anthoine (Anthoine 2000) used as a test case for the proposed model. In this section the low amplitude and moderate amplitude regimes are discussed. The model is developed in section 5.3. Succinct descriptions of the acoustic loss model and of the vortex dynamics model which are used are also made in this section. The mathematical details are given in appendix $\mathrm{H}$ and E. Predictions are compared to cold gas experimental data in section 5.4. Changes of the nozzle shape and the cavity volume $V_{c}$ are also investigated in the section. Conclusions are summarized in section 5.5.

\subsection{Cold gas model with axial injection}

Fig. 5.2 shows a sketch of the axial injection (1/30) scale model of the Ariane 5 booster used by Anthoine (Anthoine 2000). The cylindrical pipe of inner radius $r_{p}$ and length $L_{p}$ is terminated at the upstream end by a porous wall through which air is injected. This air is provided by a reservoir at a pressure $p_{r} \simeq 5 \mathrm{bar}$ and room temperature. The static pressure in the pipe is around $p=p_{r} /(1+19 M)$ (Anthoine 2000), where $M$ is the cross-sectional averaged Mach number in the pipe. In the experiments this Mach number varies in the range $0.05 \leq M \leq 0.20$. At the downstream pipe end, a submerged (integrated) nozzle is installed. This integrated nozzle has a cavity volume, $V_{c}$, representative of the acoustically accessible cavity volume which occurs during full-scale firing test. Different nozzle models are used to vary $V_{c}$ (see Table 5.1). The Mach number $M$ in the pipe can be varied by reducing the nozzle throat area by the introduction of a needle. A solid sharp-edged orifice of radius $r_{o}<r_{p}$ is placed at a distance $L_{o}$ from the nozzle inlet. This orifice represents a thermal inhibitor similar to those emerging from the propellant in a full-scale SRM, and acts as a vortex generator.

Anthoine (Anthoine 2000) considered various models of the cavity corresponding to various stages during a firing test. In the present discussion, the focus is on the data for the largest cavities (nozzles 1,7 and 9 in Table 5.1). Anthoine et al. (Anthoine et al. 2002) observed that the pulsation level $p_{\text {rms }} / p$ measured at the upstream head-end wall was proportional to the cavity volume, $V_{c}$. This is predicted by the theory, if it is assumed that the sound is generated when the vortices travel along the cavity opening and not by the ingestion of the vortices 
by the nozzle.

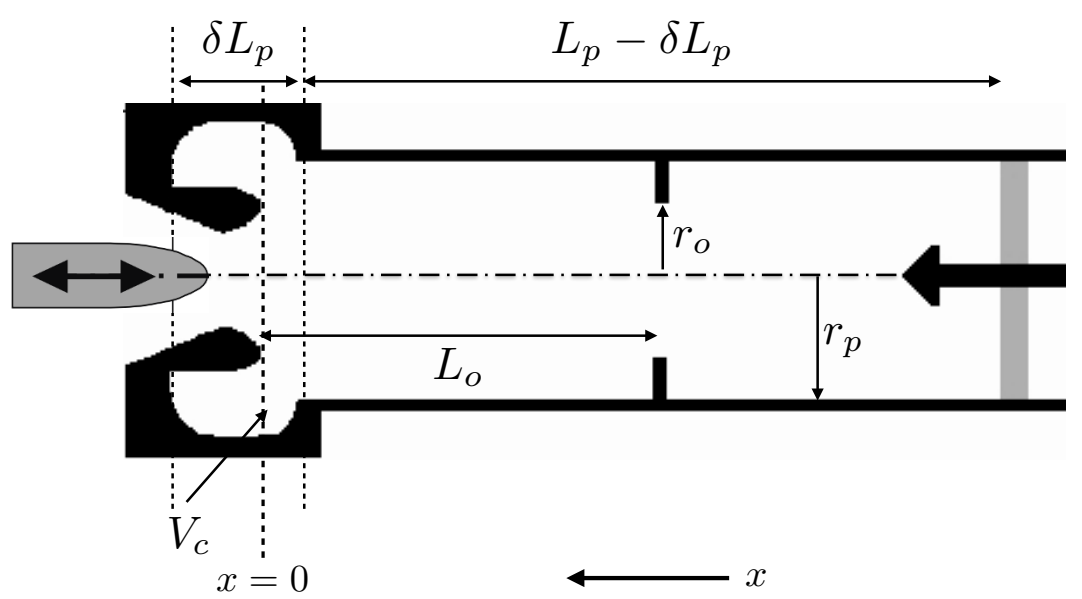

Figure 5.2: Axial injection cold gas scale experimental setup. The nozzle inlet is positioned at $x=0$. The positive flow direction is from right to left. Air is injected through the upstream porous head end wall (light grey), the injection is indicated with a big black arrow pointing to the nozzle on the axis of symmetry. The inlet Mach number $M$ is varied by introduction of a needle (dark grey) in the nozzle throat.

A typical result of experiments by Anthoine (Anthoine 2000) is shown in Fig. 5.3 for $L_{p}=0.393 \mathrm{~m}, L_{0}=0.071 \mathrm{~m}, r_{p}=0.038 \mathrm{~m}$ and $r_{0}=0.029 \mathrm{~m}$. The measured root-mean-square amplitude of the pressure pulsations, $p_{\text {rms }}$, measured at the head end, and normalized with static pressure $p$, is shown as a function of the Mach number $M$. In applications with combustion such, as in (Dotson et al. 1997) the Mach number decreases as a function of time because for a fixed throat diameter, the nozzle inlet diameter increases in time.

The oscillation frequency, $f$, is shown in dimensionless form $f / f_{n}$ in Fig. 5.3. For each acoustic mode, the oscillation frequency increases monotonically with increasing Mach number. The oscillation frequencies are close to the acoustic modes of a closed-closed pipe of length $\mathfrak{L}_{\text {eff }}$ :

$$
f_{n}=n \frac{c}{2 \mathfrak{L}_{\mathrm{eff}}},
$$

with $n=1,2,3 \ldots$ where $\mathrm{c}$ is the speed of sound in the pipe and

$$
\mathfrak{L}_{\text {eff }} \equiv L_{p}-\delta L_{p}+\frac{V_{c}}{\pi r_{p}^{2}}
$$

where $V_{c}$ is the nozzle cavity volume and $\delta L_{p}$ is the depth of the nozzle cavity. Thus, as a first low-frequency approximation, the acoustic effect of the cavity is 


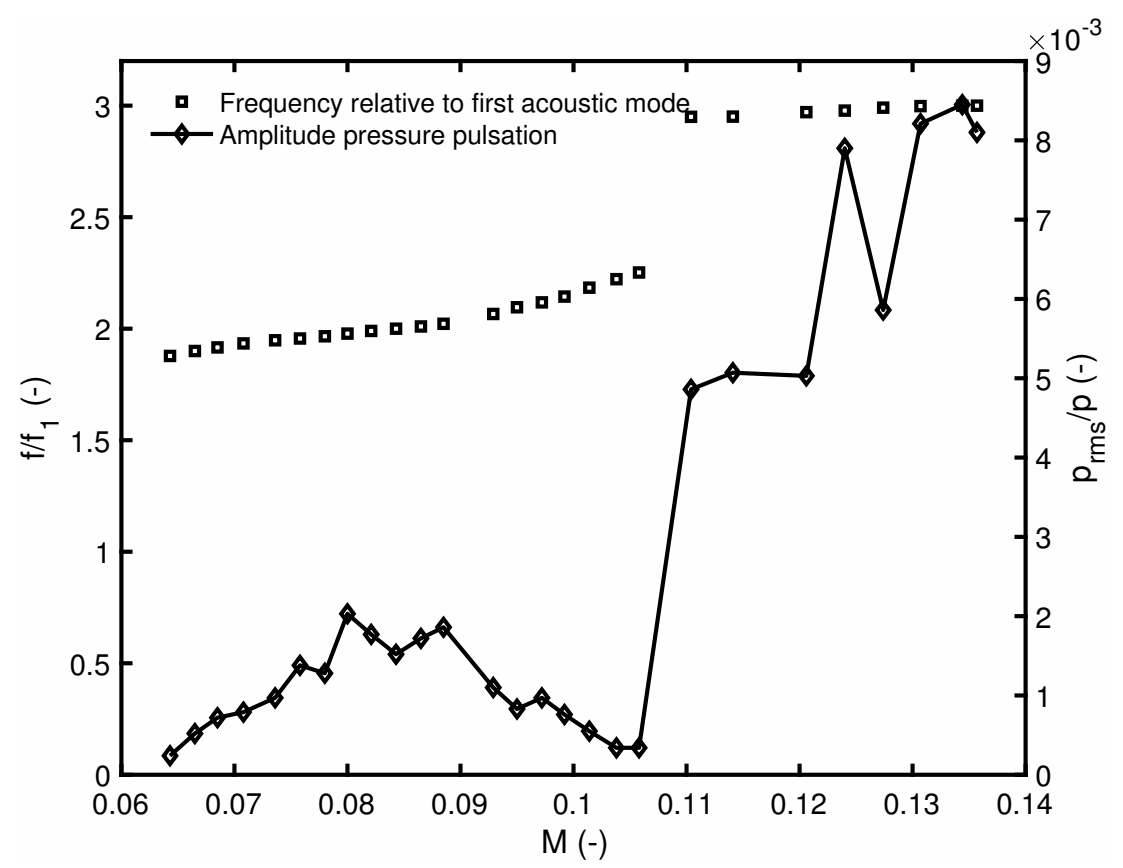

Figure 5.3: Pulsation frequency with respect to the acoustic mode frequency (left) and relative rms pressure oscillation level (right) as a function of the Mach number. Figure after figure 4.27a in (Anthoine 2000).

taken into account by increasing the pipe length by $V_{c} /\left(\pi r_{p}^{2}\right)$. The influence of the orifice on the acoustic mode frequency is neglected here. The mean pressure loss across the porous head end wall is large enough to approximate it as a solid wall for the calculation of the acoustic mode frequency. However, the effect of porosity on the acoustic losses is accounted for in the next section. Note that in the acoustic model the head end wall is at $x=-\mathfrak{L}_{\text {eff }}$.

Due to flow separation at the sharp edges of the orifice, a free jet develops downstream of the orifice with a radius $r_{\text {jet }}$ smaller than the orifice radius $r_{o}$. The ratio $\left(r_{\text {jet }} / r_{o}\right)^{2} \equiv \Upsilon$ is called the vena contracta factor. It is a function of $r_{o} / r_{p}$. Empirical data by Gilbarg (Gilbarg 1960) is used to determine this vena contracta factor. Here dependence of the vena contracta factor on the Mach number is neglected (Hofmans et al. 2001). For the cases considered by Anthoine (Anthoine 2000; Yildiz 2000), the pipe radius is $r_{p}=0.038 \mathrm{~m}$ and three different orifice radii were used $r_{o}=0.029 \mathrm{~m}, 0.031 \mathrm{~m}$ and $0.034 \mathrm{~m}$. The vena contracta factors are in these cases are $\Upsilon=0.68,0.71,0.78$, respectively.

The fluctuation of the acoustic velocity at the edge of the orifice, induces a modulation of the vorticity in the shear layer bounding the jet. This triggers a hydrodynamic instability of the shear layer, and the formation of coherent vortical structures (vortices). An empirical estimate of the convection velocity, 
$U_{\Gamma}$, of these vortices is $U_{\Gamma}=0.4 U_{\text {jet }}=0.4 U\left(r_{p} / r_{\text {jet }}\right)^{2}$ (Bruggeman et al. 1991; Hirschberg et al. 2016) where $U=M c$. Using this estimate, one can estimate the number of vortices, $m_{h}$, between the orifice and the nozzle inlet, as:

$$
m_{h}=\frac{f_{n} L_{o}}{U_{\Gamma}}=\frac{\Upsilon}{0.4 M}\left(\frac{n}{2}\right)\left(\frac{L_{o}}{\mathfrak{L}_{\text {eff }}}\right)\left(\frac{r_{o}}{r_{p}}\right)^{2} .
$$

Maxima in pulsation amplitudes appear to correspond to an integer number of vortices $m_{h}$, which is called a hydrodynamic mode. Each hydrodynamic mode has a Mach number $M=U / c$ for which the pulsation amplitude reaches a maximum and the frequency coincides with an eigenfrequency $f_{n}$ of an acoustic mode $n$ given by Eq. (5.1). Around the eigenfrequency $f_{n}$, the frequency $f$ of the oscillation increases with increasing Mach number as shown by Fig. 5.1 and 5.3.

In the present work, a 2-D incompressible planar potential flow model is used to calculate the vortex velocity and path. Assuming a standing wave, one can estimate the relative acoustic velocity fluctuations, $u_{\mathrm{rms}} / U$, upstream of the orifice using:

$$
\frac{u_{\mathrm{rms}}}{U}=\frac{p_{\mathrm{rms}}}{\gamma p M}\left|\sin \left(k_{n} x_{o}\right)\right|,
$$

where $p_{\text {rms }}$ corresponds to the pressure fluctuations at the anti-node at the upstream head end, and $\gamma=1.4$ is the ratio between the heat capacity at constant pressure and the heat capacity at constant volume. In Eq. (5.3), $k_{n}=2 \pi f_{n} / c$ is the wavenumber and $x_{o}=L_{o}+V_{c} /\left(\pi r_{p}^{2}\right)$ is the effective position of the orifice. The maximum of the observed pressure pulsation level is in the range $10^{-3} \leq p_{\text {rms }} / p \leq 10^{-2}$. Hence this corresponds for $M=\mathcal{O}\left(10^{-1}\right)$ to velocity fluctuations of the order $10^{-2} \leq u_{\mathrm{rms}} / U \leq 10^{-1}$. These are moderate amplitude levels according to the classification of Bruggeman et al. (Bruggeman et al. 1991; Tonon et al. 2011). Further downstream, the shear layer breaks down into discrete vortices. One new vortex is formed each time the acoustic velocity in the flow just upstream of the inhibitor passes through a zero amplitude, while increasing to positive values. This is the phase condition proposed by Nelson et al. (Nelson et al. 1983), and Bruggeman et al. (Bruggeman et al. 1991), predicts moderate-amplitude behavior due to low Mach number self-sustained flow instabilities in cavities and pipe systems.

While the phase of the vortex shedding is controlled by the acoustic field, the magnitude of the circulation of the vortex is independent of the acoustic amplitude. The circulation $\Gamma_{\max }$, corresponding to the amount of vorticity shed during one period of oscillation, is an upper bound for the circulation, 
$\Gamma$, of the vortices that reach the nozzle. Once a new vortex is formed at time $t=\tau$, its circulation $\Gamma$ is assumed to grow linearly in time over one period of oscillation $2 \pi / \omega$, until it reaches maturity and its circulation remains constant. The present model assumes for $t-\tau<2 \pi / \omega$ that:

$$
\Gamma=K \frac{\Gamma_{\max } \omega}{2 \pi}(t-\tau)
$$

where

$$
\Gamma_{\max }=0.5 U_{\text {jet }}^{2} \frac{2 \pi}{\omega}
$$

where $U_{\text {jet }}=M c / \Upsilon$ is the flow velocity in the jet formed by flow separation at the orifice. $K$ is a constant, intended to describe the reduction of the circulation of the coherent structures due to turbulent diffusion.

When using this model with $K=1$, and assuming that all the vorticity was concentrated into a line vortex, Bruggeman et al. (Bruggeman et al. 1991) and Tonon et al. (Tonon et al. 2011) found pulsation amplitudes in pipe systems with closed side branches which were about a factor five too high. In axially symmetric numerical simulations of hot-gas scale-model experiments Gallier et al. (Gallier et al. 2009) found a reduction of $30 \%$ to $50 \%$ of the predicted pulsation amplitude when turbulence was taken into account using a URANS model. Similarly, when considering sound production by a compact cavity in a corrugated pipe, Golliard et al. (Golliard et al. 2013) also found reduction of acoustic power by a factor two when turbulence was taken into account. Hence, one expects that $K$ should be in the range $1 / 5 \leq K \leq 1 / 2$. The parameter $K$ will be fitted to one experiment, and the ability of the resulting model to predict other experiments will be verified.

Linear stability theory for a parallel shear layer flow (Michalke 1965) shows that the growth of linear perturbations of a shear layer are limited to low frequencies, such that the hydrodynamic wavelength of the perturbation is about ten times the shear layer momentum thickness. Due to turbulence, the shear layer width increases linearly with the distance from the separation point. At low pulsation amplitudes, the perturbations may reach the critical shear layer width, for which the layer becomes linearly stable. This occurs before the vorticity has been fully concentrated into a coherent vortical structure. This explains why below a certain pulsation amplitude, the vortices have a lower circulation which is amplitude dependent. The "moderate amplitude" model fails typically for $\left|\mathbf{u}^{\prime}\right| / U<10^{-2}$ where $U=c M$. At these low amplitudes, the moderate amplitude model tends to overestimate the pulsation level. 
In the numerical simulations of Gallier et al. (Gallier et al. 2009) excellent predictions of the pulsation amplitudes are obtained with respect to most experimental results. The only exception is that the model drastically over-predicts or under-predicts the pulsation amplitude in cases for which the observed pulsations were very low. This indicates that in the low amplitude regime the pulsation amplitude is difficult to predict. In experiments, one actually observes that the pulsation amplitude in the low amplitude regime can change by orders of magnitude as a result of small modifications in the setup. At very low amplitudes, the circulation, $\Gamma$, of the vortices is proportional to the amplitude of the local acoustic velocity fluctuations where the vortices are shed. In that case, both losses and sound production depend quadratically on the pulsation amplitude, which implies that the limit-cycle amplitude is undetermined (Tonon et al. 2011).

An overview of the available experimental data is provided in Table 5.2. One observes that the pulsation amplitude is in most cases in the moderate amplitude regime. Experiments with low amplitudes are indicated by an asterisk.

\subsection{Theoretical analysis}

\subsubsection{Single degree of freedom oscillator model}

Following Howe (Howe 1998), for low Mach number flows, the acoustic field can be described in terms of solutions of d'Alembert's equation for the total enthalpy fluctuation $B^{\prime}$. As the frequency of the oscillation is low compared to the cut-on frequency for transversal pipe modes, such solutions take the form of traveling plane acoustic waves. One can also describe the solutions in terms of a series of standing waves (Culick 2004).

Here the crudest approximation is used for which only one dominating standing wave is considered; corresponding to the resonance frequency $f_{n}$. Furthermore the discussion is restricted to the limit cycle, which is assumed to be dominated by a single harmonic oscillation. This implies that the acoustic field described in terms of total enthalpy fluctuations $B^{\prime}$ is

$$
B^{\prime}=-\left|b_{n}\right| \cos \left(k_{n} x\right) \cos \left(\omega t+\Phi_{n}\right),
$$

where $k_{n}=2 \pi f_{n} / c=\omega_{n} / c$ is the wavenumber, $\omega=2 \pi f$ is the angular frequency, $\left|b_{n}\right|$ is the amplitude of the total enthalpy fluctuations and $x$ is the distance from the nozzle inlet. The phase $\Phi_{n}$ is chosen such that vortex shedding occurs at $t=0$. 
For a one-dimensional flow of time averaged velocity $U$ and velocity fluctuations $u^{\prime}$, the total enthalpy fluctuations are related to the pressure fluctuation $p^{\prime}$ by

$$
B^{\prime}=\frac{p^{\prime}}{\rho_{0}}+u^{\prime} U
$$

where $\rho_{0}$ is the density of the unperturbed mean flow. The acoustic power flux (energy per unit time and unit surface area), also called the acoustic intensity $I$, is given by the following relation (Howe 1998)

$$
I=m^{\prime} B^{\prime}
$$

where $m^{\prime}$ is the fluctuation in mass flux, i.e. one has

$$
m^{\prime}=\rho^{\prime} U+\rho_{0} u^{\prime} .
$$

The acoustic velocity $u^{\prime}$ is obtained by using the linearized equation of Euler in the form

$$
\frac{\partial u^{\prime}}{\partial t}=-\frac{\partial B^{\prime}}{\partial x} .
$$

Using Eq. (5.6), one finds:

$$
u^{\prime}=-\frac{\left|b_{n}\right|}{c}\left(\frac{\omega_{n}}{\omega}\right) \sin \left(k_{n} x\right) \sin (\omega t) .
$$

As proposed by Culick (Culick 2004), this system is described as a damped single degree of freedom harmonic oscillator driven by a source term $\mathcal{F}_{\text {vortex }}$

$$
\mathcal{M}_{\text {eff }} \frac{\mathrm{d}^{2} b_{n}}{\mathrm{~d} t^{2}}+\gamma_{\text {eff }} \frac{\mathrm{d} b_{n}}{\mathrm{~d} t}+K_{\text {eff }} b_{n}=\mathcal{F}_{\text {vortex }}
$$

The effective mass, $\mathcal{M}_{\text {eff }}$, is defined such that

$$
\frac{1}{2} \mathcal{M}_{\text {eff }}\left(\frac{\mathrm{d} b}{\mathrm{~d} t}\right)^{2}
$$

corresponds to the kinetic energy in the acoustic standing wave, viz.

$$
\mathcal{M}_{\mathrm{eff}}=\frac{4 \pi r_{p}^{2}}{\left(\omega_{n}\left|b_{n}\right|\right)^{2}}\left\langle\int_{0}^{\mathfrak{L}_{\mathrm{eff}}} \frac{1}{2} \rho_{0}\left(u^{\prime}\right)_{\omega=\omega_{n}}^{2} d x\right\rangle=\frac{\rho_{0} \pi r_{p}^{2} \mathfrak{L}_{\mathrm{eff}}}{2\left(\omega_{n} c\right)^{2}}
$$


where the brackets $\langle\cdots\rangle$ indicate time averaging over a period of oscillation. By definition, the spring constant is related to the resonance frequency $\omega_{n}=2 \pi f_{n}$ by:

$$
K_{\text {eff }}=\mathcal{M}_{\text {eff }} \omega_{n}^{2}
$$

The time-averaged acoustic power $\left\langle\mathcal{P}_{\text {diss }}\right\rangle$ dissipated at the limit cycle is given by:

$$
\left\langle\mathcal{P}_{\text {diss }}\right\rangle=\left\langle\gamma_{\text {eff }}\left(\frac{\mathrm{d} b}{\mathrm{~d} t}\right)^{2}\right\rangle
$$

where $\gamma_{\text {eff }}$ is the effective damping.

The acoustic energy produced by the vortex flow during the interaction with the nozzle is estimated from the energy corollary of Howe (Howe 1980):

$$
\left\langle\mathcal{P}_{\text {vortex }}\right\rangle=-\rho\left\langle\int_{V}(\boldsymbol{\omega} \times \mathbf{v}) \cdot \mathbf{u}^{\prime} d^{3} x\right\rangle
$$

by using a two-dimensional incompressible potential flow model assuming a line vortex $\boldsymbol{\omega}=\left(0,0, \Gamma \delta\left(x-x_{\Gamma}\right) \delta\left(y-y_{\Gamma}\right)\right)$ travelling at a velocity $\mathbf{v}$ with position $\left(x_{\Gamma}, y_{\Gamma}, 0\right)$. In Eq. (5.17), $\mathbf{u}^{\prime}$ denotes the local acoustic velocity.

\subsubsection{Linear damping}

The linear damping, $\gamma_{\mathrm{eff}}$, in Eq. (5.12) is the sum of losses due to sound radiation at the nozzle $\gamma_{\text {nozzle }}$, vortex shedding at the orifice $\gamma_{\text {orifice }}$ and viscous dissipation in the porous wall $\gamma_{\text {porous }}$ :

$$
\gamma_{\text {eff }}=\gamma_{\text {nozzle }}+\gamma_{\text {orifice }}+\gamma_{\text {porous }}
$$

In the axial flow injection case, the visco-thermal damping along the pipe wall appears to be negligible compared to the other contributions. Models for these losses are described in (Hirschberg et al. 2016) and are summarized in appendix $\mathrm{H}$.

\subsubsection{Vortex path}

The flow model used to predict the vortex path and its interaction with the local acoustic field at the nozzle inlet is a two dimensional incompressible potential flow model. For convenience, the flow in a quarter of the channel cross-section 


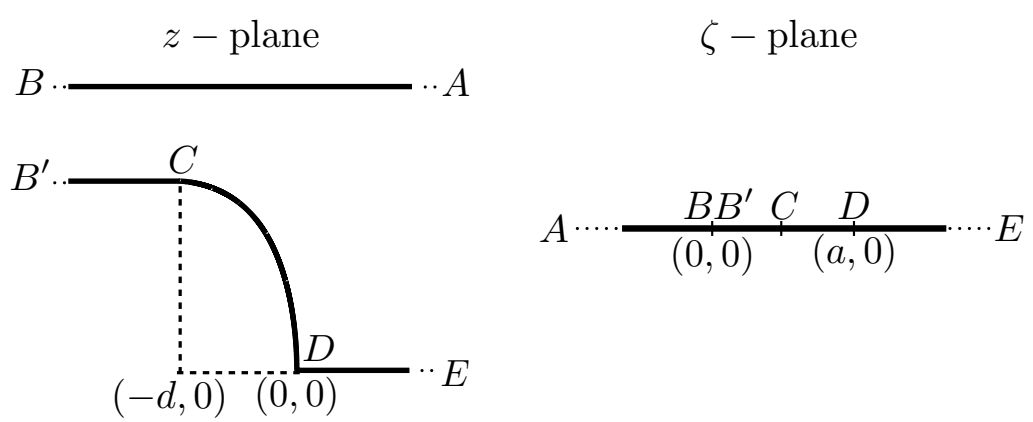

Figure 5.4: Conformal mapping, Schwarz-Christoffel transformation of backward facing step with uniform approach flow. The inner points in z-plane are mapped to the upper two quadrants in the $\zeta$-plane.

is considered. The nozzle is represented as an abrupt contraction in a two dimensional channel from a square section of width $S_{1}=\sqrt{\pi} r_{p} / 2$ to a rectangular channel of height $S_{2}$ and width $S_{1} . S_{2}$ is chosen using the formula:

$$
S_{2}=S_{1} M\left(\frac{\gamma+1}{2\left(1+\frac{\gamma-1}{2} M^{2}\right)}\right)^{\frac{\gamma+1}{2(\gamma-1)}}
$$

such that for an inlet Mach number $M$ in a section of area $S_{1}^{2}$ the channel area $S_{1} S_{2}$ after the two-dimensional contraction corresponds to a area of the choked nozzle throat, as calculated using quasi-one dimensional steady flow gas dynamics (Thompson 1972).

The vortex path is calculated using the following simplifying assumptions:

1. The vortex does not interfere with other vortices that are shed earlier or later.

2. A new vortex is shed each oscillation period when the acoustic velocity $u^{\prime}$ calculated with Eq. (5.11) vanishes at the orifice $x=x_{o}$, while heading in the direction of the main flow towards the nozzle.

3. The vortex circulation, $\Gamma$, increases linearly during the first oscillation period after it is shed, following the model of Eq. (5.4).

4. The vortex circulation, $\Gamma$, remains constant after one period of oscillation.

5. The acoustic flow does not influence the vortex path.

6. The cavity surrounding the nozzle inlet does not influence the vortex path.

7. The vortex path is not influenced by other vortices (shed earlier or later).

By using conformal mapping in the complex plane $z=x+\mathrm{i} y=f(\zeta)$ (where 
$\left.\mathrm{i}^{2} \equiv-1\right)$, the physical plane $(x, y)$ is mapped into the half plane above the real axis in the complex $\zeta$ plane as shown in Fig. 5.4.

A modified Schwarz-Christoffel transformation as proposed by Henrici is used (Henrici 1974). Details of the transformation can be found in chapter 3 and appendix D (Hirschberg et al. 2017). The Henrici transformation introduces the contraction length $d$, in the $z$ plane as shown in Fig. 5.4. Calculations are presented for a sharp square edge with $d=0$ and for a rounded nozzle inlet with $d=S_{1} / 2$. This will provide some insight into the effect of the nozzle geometry on the sound production.

The solution for the potential of the flow is obtained with the method of images. The vortex velocity and path $z_{\Gamma}$ are then obtained by standard methods. Details are provided in appendix E.

\subsubsection{Acoustic field}

The acoustic field in the pipe is approximated by a plane acoustic standing wave Eq. (5.11). A more detailed description of the local acoustic field in the vicinity of the nozzle inlet is needed when applying the energy corollary Eq. (5.17). By definition, the acoustic field is a potential flow (frictionless). In the vicinity of the nozzle inlet, there are two contributions to the acoustic velocity: the flow corresponding to the radiation of sound through the nozzle, $\mathbf{u}_{n}^{\prime}$, and the flow induced by the acoustic compression of the gas in the cavity surrounding the nozzle inlet $\mathbf{u}_{c}^{\prime}$. At the low frequencies considered here, one can assume a quasi-steady acoustic flow through the nozzle. This is, in the incompressible potential flow, model a harmonically oscillating flow induced by a line (2-D point) source positioned at $\zeta=0$ corresponding to points $B$ and $B^{\prime}$ in Fig. 5.4. The source strength $Q_{n}$ is given by :

$$
Q_{n}=\frac{\gamma-1}{2} M \frac{\left|b_{n}\right|}{c} S_{1} \cos \left(\omega t+\Phi_{n}\right),
$$

with a scalar potential

$$
\phi_{n}=\frac{Q_{n}}{\pi} \ln (\zeta)
$$

The acoustic field induced by the cavity is represented by a line source, $Q_{c}$, of length $S_{1}$ positioned at the corner just before the contraction of the nozzle inlet (point $D$ in Fig. 5.4). Fig. 5.4 shows the cross-sectional two dimensional plane out of which the line source protrudes. Point $D$ is at $z=0$ which corresponds to $\zeta=a$ (see E). As the volume of the cavity, $V_{c}$, is small compared to the acoustic 
wavelength, one can assume a uniform pressure in the cavity and estimate $Q_{c}$ from an integral mass balance, neglecting the steady flow velocity

$$
Q_{c}=-\frac{V_{c}}{4 S_{1} \rho_{0} c^{2}} \frac{\mathrm{d} p^{\prime}}{\mathrm{d} t}=-\frac{V_{c}}{4 S_{1} c^{2}} \frac{\mathrm{d} b_{n}}{\mathrm{~d} t}
$$

this is associated with the scalar potential

$$
\phi_{c}=\frac{Q_{c}}{\pi} \ln (\zeta-a) .
$$

In order to investigate the effect of the geometry of the cavity opening, some calculations were carried out in which the volume flux $Q_{c}$ was distributed between two line sources, placed along the real axis in the $\zeta$-plane.

The acoustic velocity (in complex notation) in the $z$-plane is given by:

$$
u^{\prime}=\left[\frac{\mathrm{d}\left(\phi_{n}+\phi_{c}\right)}{\mathrm{d} z}\right]^{*}=\left[\frac{\mathrm{d}\left(\phi_{n}+\phi_{c}\right)}{\mathrm{d} \zeta} \frac{1}{\left(\frac{\mathrm{d} f}{\mathrm{~d} \zeta}\right)_{\zeta_{L}}}\right]^{*}
$$

where $(\ldots)^{*}$ denotes the complex conjugate.

\subsubsection{Energy balance}

The power, $\mathcal{P}_{\text {vortex }}$, (Eq. (5.17)) generated by a line vortex of circulation $\Gamma$, of length $2 S_{1}$ and position $z_{\Gamma}$ is given in complex notation by (Peters 1993)

$$
\mathcal{P}_{\text {vortex }}(t)=-4 S_{1} \operatorname{Re}\left[\mathrm{i} \rho_{0} \Gamma\left(u^{\prime}\right)^{*} \frac{\mathrm{d} z_{\Gamma}}{\mathrm{d} t}\right]=-4 S_{1} \operatorname{Re}\left[\mathrm{i} \rho_{0} \Gamma\left(u^{\prime}\right)^{*} \frac{\mathrm{d} \zeta_{\Gamma}}{\mathrm{d} t}\left(\frac{\mathrm{d} f}{\mathrm{~d} \zeta}\right)_{\zeta_{\Gamma}}\right]
$$

As the vortex position, $\zeta_{\Gamma}$, and its velocity, $\mathrm{d} \zeta_{\Gamma} / \mathrm{d} t$, are known in the $\zeta$-plane as a function of the time, the power generated by a vortex can be obtained. The time-averaged power generated by the vortices is calculated as

$$
\left\langle\mathcal{P}_{\text {vortex }}\right\rangle=\frac{\omega}{2 \pi} \int_{0}^{t_{\text {max }}} \mathcal{P}_{\text {vortex }}(t) \mathrm{d} t .
$$

where the origin of time is taken to be the moment at which the vortex is shed from the orifice at $z_{0}$, and $t_{\max }$ is a time after which the vortex has passed the contraction and no longer produces sound (as its path is parallel to the acoustic 
streamlines in the channel). When there is more than one vortex between the orifice and the nozzle, this integration-time is larger than one period of oscillation $2 \pi / \omega$. The contribution of all vortices within one period of oscillation equals the contribution of a single vortex over its entire active life $0<t<t_{\max }$.

Assuming the oscillation frequency to correspond to the eigenfrequency of a mode, $\omega=\omega_{n}$, the energy balance is given by

$$
\left\langle\mathcal{P}_{\text {vortex }}\right\rangle=\left\langle\gamma_{\text {eff }}\left(\frac{\mathrm{d} b_{n}}{\mathrm{~d} t}\right)^{2}\right\rangle,
$$

where $\left\langle\mathcal{P}_{\text {vortex }}\right\rangle$ is calculated using Eq. (5.25) combined with Eq. (5.26). This yields a first-order equation for the amplitude, $\left|b_{n}\right|$, of the standing wave in the pipe. Indeed the energy losses scale with the square of the amplitude $\left|b_{n}\right|^{2}$, while the sound production scales with the amplitude $\left|b_{n}\right|$. This is a consequence of the assumption that both the circulation, $\Gamma$, and the vortex path are assumed to be independent of the amplitude of the acoustic perturbation. In the model, only the phase of the vortex shedding at the orifice is determined by the acoustic oscillation.

\subsubsection{Influence of Mach number on frequency}

The energy balance model does not allow the prediction of the variation of oscillation frequency with the Mach number $M$ as observed in Figures 5.1 and 5.3. A lumped element model is developed here for the purpose of finding an equation for the deviation of the frequency $\omega$ with respect the resonance frequency $\omega_{n}$. The derived equation will be used to estimate the slope $(M / \omega) \mathrm{d} \omega / \mathrm{d} M$.

For a low Mach number homentropic flow with main steady velocity field, $\mathbf{U}$, in an acoustically closed-closed pipe, the vortex sound analogy (chapter 2 Eq. (2.17)) can be written as

$$
\frac{1}{c^{2}} \frac{\partial^{2} B^{\prime}}{\partial t^{2}}-\nabla^{2} B^{\prime}=\nabla \cdot(\boldsymbol{\omega} \times \mathbf{v})-\frac{1}{\rho_{0}} \mathbf{U} \cdot \nabla\left(\frac{\partial B^{\prime}}{\partial t}\right) .
$$

Using Green's function, $G(\mathbf{x}, \mathbf{y}, t-\tau)$, developed in $\operatorname{modes}^{2} \psi_{n}$ (Eq. (2.19))and neglecting second order terms in the Mach number, one finds a set of ordinary differential equations for the mode amplitudes:

$$
\frac{\mathrm{d}^{2} b_{n}}{\mathrm{~d} t^{2}}+\frac{\gamma_{\mathrm{eff}}}{\mathcal{M}_{\mathrm{eff}}} \frac{\mathrm{d} b_{n}}{\mathrm{~d} t}+\omega_{n}^{2} b_{n}=-\left(\frac{c}{E_{n}}\right)^{2} \int_{V}(\boldsymbol{\omega} \times \mathbf{v}) \cdot \nabla_{\mathbf{y}} \psi_{n} \mathrm{~d}^{3} y .
$$

\footnotetext{
${ }^{2}$ Where $\psi_{n}$ are solutions of the Helmholtz equation Eq. (2.20), with boundary conditions $\nabla \psi_{n} \cdot \mathbf{n}=0$.
} 
For a stable limit cycle, $b_{n}=\left|b_{n}\right| \cos \left(\omega t+\Phi_{n}\right)$. The phase $\Phi_{n}$, is chosen such that $t=0$ corresponds to the moment at which a new vortex is shed. Substitution in Eq. (5.29) and multiplying by $\sin \left(\omega t+\Phi_{n}\right)$ one obtains, by averaging over a period of oscillation:

$$
\left|b_{n}\right|=-\frac{2 \mathcal{M}_{\text {eff }}}{\gamma_{\text {eff }}}\left(\frac{c}{E_{n}}\right)^{2}\left\langle\int_{V}(\boldsymbol{\omega} \times \mathbf{v}) \cdot \nabla_{\mathbf{y}} \psi_{n} \mathrm{~d}^{3} y \sin \left(\omega t+\Phi_{n}\right)\right\rangle .
$$

Multiplying by $\cos \left(\omega t+\Phi_{n}\right)$ and time averaging over one period of oscillation yields a second equation for $\left|b_{n}\right|$. Combining with Eq. 5.30, one finds

$$
\omega_{n}-\omega=\frac{\gamma_{\mathrm{eff}}}{\mathcal{M}_{\mathrm{eff}}\left(\omega+\omega_{n}\right)} \frac{\left\langle\int_{V}(\boldsymbol{\omega} \times \mathbf{v}) \cdot \nabla_{\mathbf{y}} \psi_{n} \mathrm{~d}^{3} y \cos \left(\omega t+\Phi_{n}\right)\right\rangle}{\left\langle\int_{V}(\boldsymbol{\omega} \times \mathbf{v}) \cdot \nabla_{\mathbf{y}} \psi_{n} \mathrm{~d}^{3} y \sin \left(\omega t+\Phi_{n}\right)\right\rangle} .
$$

When substituting the approximation $\omega=\omega_{n}$ into Eq. (5.30), one obtains the same expression for $\left|b_{n}\right|$ as obtained with the energy balance model in the case where sound is only generated by the interaction of a vortex with the acoustic field generated by the cavity $V_{c}$. This implies that when sound production due to ingestion of a vortex by the nozzle is neglected.

The gradient of the eigenfunction, $\nabla \psi_{n}$, describes the acoustic flow in the combustion chamber including the effect of the cavity volume $V_{c}$. However, a very crude approximation for the Green's function, $G$, is used. Namely, a loss free boundary condition $\mathbf{n} \cdot \nabla \psi_{n}=0$ is used for the modes $\psi_{n}$ in the expansion of $G$. This approximation does not account for the local acoustic velocity field induced by radiation through the nozzle. This is a problem for the vortex-nozzle interaction sound source due to the $\nabla G_{r}$ component discussed in chapter 4.4.1. However, as discussed in chapter 4.12 the compressible component $\nabla G_{c}$ is dominant. Furthermore, it should be noted that the energy losses due to radiation are correctly described (Eq. (2.38)) and appear in $\gamma_{\text {eff }}$.

Substituting $\omega=\omega_{n}$ in the right hand side of Eq. (5.31), yields an estimate for the deviation $\omega_{n}-\omega$ of the oscillation frequency $\omega$ from the resonance frequency $\omega_{n}$. This approximation is used to obtain a theoretical value of the slope $(M / \omega) \mathrm{d} \omega / \mathrm{d} M$ of the frequency as a function of the Mach number $M$ around a pulsation maximum. A more accurate solution can be obtained by successive substitution of approximations of $\omega$ (Culick 2006). Only the initial approximation described above is considered here. It is obvious from this approximation that the slope is a measure for the quality factor $\mathcal{Q} \equiv \mathcal{M}_{\text {eff }} \omega_{n} / \gamma_{\text {eff }}$ of the resonator. 
Table 5.1: Nozzle numbers (column 1) and associated cavity volumes $V_{c}$ (column 2) used in cold gas experiments at the VKI (Anthoine 2000; Anthoine and Lema 2009; Yildiz 2000). The scaling factor $\alpha \equiv V_{\text {nozzle }} 7 / V_{c}$ is shown in column 3 .

\begin{tabular}{c|c|c} 
Nozzle & $V_{c}$ & $\alpha$ \\
- & $\mathrm{m}^{3} \times 10^{-5}$ & - \\
\hline 1 & 20.3 & 0.906 \\
2 & 10.0 & 1.84 \\
5 & 6.71 & 2.74 \\
7 & 18.4 & 1.00 \\
9 & 21.8 & 0.844
\end{tabular}

\subsection{Results}

\subsubsection{Comparison of model with experiments}

Table 5.1 contains the nozzle numbers, as defined by Anthoine (Anthoine 2000) and their associated cavity volumes, $V_{c}$, used in cold gas scale experiments carried out at the von Karman Institute (VKI).

In the following tables, experimental data are given for maxima of pulsation levels observed as a function of the Mach number. An overview of the pulsation maxima in all the available axial injection experiments is shown in Table 5.2. For all the experiments, the pipe radius, $r_{p}$, is fixed at $r_{p}=3.8 \times 10^{-2} \mathrm{~m}$. In column 8 of Table 5.2, the estimated acoustic mode number, $n$, of the experiments is shown. The estimated hydrodynamic mode number, $m_{h}$, is calculated using Eq. (5.2). The results for $m_{h}$ are shown in column 9 of Table 5.2. It is noteworthy that most of the experiments are in the second hydrodynamic mode, for which $m_{h} \simeq 2$. This means that in most experiments two vortices, with a phase delay of a period of oscillation between them, are traveling simultaneously from the orifice to the nozzle. For measurements 8 and 19 (Table 5.2 ), both of which are associated with a small distance $L_{o}=4.6 \times 10^{-2} \mathrm{~m}$ between the orifice and the nozzle inlet, a hydrodynamic mode number $m_{h} \simeq 1$ is observed. This means that a single vortex is present between the orifice and the nozzle in these experiments.

The relative acoustic velocity fluctuation $u_{\mathrm{rms}} / U$ at the orifice $\left(x=x_{o}\right)$ is calculated with Eq. (5.3). The results are shown in column 10 of Table 5.2. If $10^{-2} \leq u_{\mathrm{rms}} / U \leq 10^{-1}$, the measurement is in the moderate amplitude regime (Bruggeman et al. 1991). The model described in this paper is valid for this regime. If this relative fluctuation in acoustic velocity is $\mathcal{O}\left(10^{-3}\right)$, it is in the so-called low-amplitude regime (Bruggeman et al. 1991), in which case the proposed model is not valid. These experiments are marked with an asterisk e.g. $5^{*}$. One will indeed observe that the theory fails for these experiments. 
The influence of the cavity volume $V_{c}$ used in experiments is shown in Table 5.3. All experiments in Table 5.3 have a fixed pipe length $L_{p}=0.393 \mathrm{~m}$, distance between the orifice and the nozzle inlet $L_{o}=7.1 \times 10^{-2} \mathrm{~m}$, orifice radius $r_{o}=2.9 \times 10^{-2} \mathrm{~m}$ and vena contracta factor $\Upsilon=0.68$.

It is expected that the relative pressure pulsations are proportional to the cavity volume, $V_{c}$, used in the experiment (Anthoine 2000). Thus the experimental data are scaled by $V_{c}$. The pulsation amplitude $\left(p_{\text {rms }} / p\right)_{\exp }$ is multiplied by the factor:

$$
\alpha \equiv \frac{V_{\text {nozzle } 7}}{V_{c}} .
$$

In column 3 of Table 5.1 the scaling factor $\alpha$ is shown for all nozzles considered in this text. The resulting scaled relative pressure pulsations are shown in column 6 of Table 5.3.

In the last two columns of Table 5.3 the experiments are compared to model, using the fit parameter $K=1 / 3$. To determine the pulsation maximum predicted using the energy balance model, the following steps are taken. An initial estimate of the Mach number is taken near the Mach number that produced a pressure oscillation maximum $\left(p_{\mathrm{rms}} / p\right)_{\exp }$ in the experiments. The relative pulsation amplitude $\left(p_{\text {rms }} / p\right)_{\text {th }}$ is calculated using the energy balance. The Mach number is increased by an increment, then the relative pulsation amplitude is again calculated using the energy balance. This is repeated until a maximum in relative pulsation amplitude is reached. If none is reached, the same process is repeated with a decreasing increment in Mach number starting from the initial estimated Mach number.

In Table 5.3, one observes that experiments taken from (Anthoine 2000) are reproduced with the model for $n=2$ within $10 \%$, for both Mach numbers and relative pressure pulsation amplitudes. For $\mathrm{n}=3$ the deviation of $30 \%$ between the model and experiments is comparable to the deviation between experiments using different nozzles.

Fig. 5.5 shows the measurements from Anthoine (Anthoine 2000) as a function of the Mach number for the second acoustic mode $n=2$. The upright triangles indicate data obtained with nozzle 1 , the circles nozzle 2 , squares correspond to data for nozzle 7 and sideways triangles nozzle 9 . These data are scaled using the multiplication factor $\alpha=V_{\text {nozzle } 7} / V_{c}$ since a proportionality of the pressure pulsations to $V_{c}$ is expected (Anthoine 2000). This scaling allows to collapse the data to some extent. One observes a deviation of approximately $20 \%$ between the maxima of the pulsation amplitude. 
Table 5.2: Overview of the results of experiments, with axial injection obtained with the cold flow scale model of the VKI. In the tables repeating values in columns are replaced by a dashed line.

(a) Analysis and overview of relevant experimental data from (Anthoine 2000).

\begin{tabular}{c|c|ccc|c|ccc} 
Num. & Nozzle & $L_{p}$ & $L_{o}$ & $r_{o}$ & $\Upsilon$ & $n$ & $m_{h}$ & $\frac{u_{\text {rms }}}{U}$ \\
- & - & $\mathrm{m}$ & $\mathrm{m} \times 10^{-2}$ & $\mathrm{~m} \times 10^{-2}$ & - & - & - & $-\times 10^{-2}$ \\
\hline \hline 1 & 7 & 0.393 & 7.1 & 2.9 & 0.68 & 2 & 2.1 & 1.8 \\
2 & $\mid$ & 0.393 & $\mid$ & $\mid$ & $\mid$ & 3 & 1.8 & 2.6 \\
3 & $\mid$ & 0.305 & $\mid$ & $\mid$ & $\mid$ & 2 & 1.9 & 2.0 \\
4 & $\mid$ & 0.188 & $\mid$ & $\mid$ & $\mid$ & 1 & 2.2 & 1.2 \\
$5^{*}$ & $\mid$ & 0.188 & $\mid$ & $\mid$ & $\mid$ & 2 & 2.8 & 0.65 \\
6 & $\mid$ & 0.163 & 7.1 & $\mid$ & $\mid$ & 1 & 2.0 & 1.1 \\
$7^{*}$ & $\mid$ & 0.393 & 12.6 & $\mid$ & $\mid$ & 1 & 2.1 & 0.47 \\
8 & $\mid$ & $\mid$ & 4.6 & $\mid$ & $\mid$ & 2 & 0.85 & 7.2 \\
9 & $\mid$ & $\mid$ & 4.6 & 2.9 & 0.68 & 3 & 2.1 & 1.5 \\
10 & $\mid$ & $\mid$ & 7.1 & 3.1 & 0.72 & 2 & 1.9 & 2.3 \\
$11^{*}$ & $\mid$ & $\mid$ & $\mid$ & 3.4 & 0.79 & 1 & 2.3 & 0.45 \\
12 & 7 & $\mid$ & $\mid$ & 3.4 & 0.79 & 2 & 2.0 & 1.2 \\
13 & 1 & $\mid$ & $\mid$ & 2.9 & 0.68 & $\mid$ & 2.1 & 2.1 \\
14 & 2 & $\mid$ & $\mid$ & $\mid$ & $\mid$ & $\mid$ & 2.3 & 1.2 \\
15 & 9 & $\mid$ & $\mid$ & $\mid$ & $\mid$ & 2 & 2.0 & 1.9 \\
16 & 9 & $\mid$ & $\mid$ & $\mid$ & $\mid$ & 3 & 1.8 & 4.6 \\
$17^{*}$ & 5 & $\mid$ & 7.1 & $\mid$ & $\mid$ & 2 & 2.3 & 0.71 \\
18 & 1 & $\mid$ & 4.6 & $\mid$ & $\mid$ & 3 & 2.3 & 1.9 \\
19 & 1 & 0.393 & 4.6 & 2.9 & 0.68 & 2 & 1.2 & 2.4
\end{tabular}

(b) Overview and analysis of experimental data from (Yildiz 2000).

\begin{tabular}{c|c|ccc|c|ccc} 
Num. & Nozzle & $L_{p}$ & $L_{o}$ & $r_{o}$ & $\Upsilon$ & $n$ & $m_{h}$ & $\frac{u_{\mathrm{rms}}}{U}$ \\
- & - & $\mathrm{m}$ & $\mathrm{m} \times 10^{-2}$ & $\mathrm{~m} \times 10^{-2}$ & - & - & - & $-\times 10^{-2}$ \\
\hline \hline 20 & 9 & 0.393 & 4.6 & 2.9 & 0.68 & 2 & 0.78 & 4.8 \\
21 & $\mid$ & $\mid$ & $\mid$ & 2.9 & 0.78 & 3 & 2.2 & 3.8 \\
22 & $\mid$ & $\mid$ & $\mid$ & 3.1 & 0.72 & 2 & 2.4 & 2.4 \\
23 & $\mid$ & $\mid$ & $\mid$ & 3.1 & 0.72 & 3 & 2.0 & 4.4 \\
24 & $\mid$ & $\mid$ & $\mid$ & 3.4 & 0.79 & 2 & 1.9 & 3.0 \\
25 & 9 & 0.393 & 4.6 & 0.68 & 0.79 & 3 & 1.9 & 5.1
\end{tabular}


Table 5.3: Influence of cavity volume $V_{c}$ for fixed pipe length $L_{p}=0.393 \mathrm{~m}$, orifice length $L_{o}=7.1 \times 10^{-2} \mathrm{~m}$, orifice radius $r_{o}=2.9 \times 10^{-2} \mathrm{~m}$ and vena contracta factor $\Upsilon=0.68$. In this table repeating values in columns are replaced by a dashed line.

\begin{tabular}{c|c|c|ccc|cc} 
Num. & Nozzle & $V_{c}$ & $n$ & $M_{\exp }$ & $\alpha\left(\frac{p_{\text {rms }}}{p}\right)_{\exp }$ & $\frac{M_{t h}}{M_{\exp }}$ & $\frac{\left(p_{\text {rms }}\right)_{t h}}{\left(p_{\mathrm{rms}}\right)_{\exp }}$ \\
- & - & $\mathrm{m}^{3} \times 10^{-5}$ & - & $-\times 10^{-2}$ & $-\times 10^{-3}$ & - & - \\
\hline \hline 1 & 7 & 18.4 & 2 & 8.0 & 2.0 & 0.94 & 1.0 \\
13 & 1 & 20.3 & $\mid$ & 7.9 & 2.2 & 0.95 & 0.91 \\
14 & 2 & 10.0 & $\mid$ & 7.8 & 2.4 & 1.0 & 0.93 \\
15 & 9 & 21.8 & $\mid$ & 8.6 & 1.9 & 0.87 & 1.0 \\
$17^{*}$ & 5 & 6.71 & 2 & 7.9 & 2.2 & 1.0 & 1.0 \\
\hline 2 & 7 & 18.4 & 3 & 14 & 9.3 & 0.83 & 1.1 \\
16 & 9 & 21.8 & 3 & 14 & 17 & 0.81 & 0.69
\end{tabular}

To investigate the influence of the cavity volume, $V_{c}$, two lines are shown in Fig. 5.5. The solid line corresponds to the model with nozzle 7 for which the scaling factor is $\alpha=1$. The dashed line corresponds to the model with nozzle $2, \alpha=V_{\text {nozzle } 7} / V_{\text {nozzle2 }}=1.84$. Both model predictions are made with $K=1 / 3$. Both predict the pressure pulsation quite well. The Mach number of the maximum is reproduced within $10 \%$ and the maximum pressure pulsation amplitude is reproduced within $10 \%$.

In Fig. 5.5, between results for $\alpha=1$ and $\alpha=1.84$ one observes a shift in Mach number where the maximum relative pressure pulsation (where $f=f_{n}$ ) occurs for the model. This is due to the fact that at the pulsation maximum, the hydrodynamic mode number, $m_{h}$, is fixed. As $V_{\text {nozzle7 }}>V_{\text {nozzle2 }}$ the effective length $\mathfrak{L}_{\text {eff }}$ for nozzle 7 is larger than for nozzle 2 . Thus the resonance frequency $f_{n}$ (which is inversely proportional to $\mathfrak{L}_{\text {eff }}$ ) for nozzle 7 is lower than for nozzle 2. Hence, the Mach number predicted for the maximum pressure pulsation is lower for nozzle 7 than for nozzle 2 , which keeps $m_{h}=f_{n} L_{o} /(0.4 c M)$ constant. Note that the predicted shift is not observed in the experiments.

Fig. 5.6 shows scaled data obtained by Anthoine (Anthoine 2000) for the third estimated acoustic mode, $n=3$. In this figure, squares indicate data obtained with nozzle 7 , and sideways triangles those found for nozzle 9 . The solid line in Fig. 5.6 are predictions obtained using the model, with $V_{c}=V_{\text {nozzle } 7}$ and $K=1 / 3$. Both for the empirical data and the model prediction, the dimensions are $L_{p}=0.393 \mathrm{~m}, L_{o}=0.071 \mathrm{~m}, r_{o}=0.029 \mathrm{~m}$ and $\Upsilon=0.68$.

Looking at Fig. 5.6, one observes that the empirical data for $n=3$ do not collapse. There is at least a $50 \%$ deviation between the points obtained with nozzle 7 and nozzle 9 , respectively. The model predicts an intermediate ampli- 


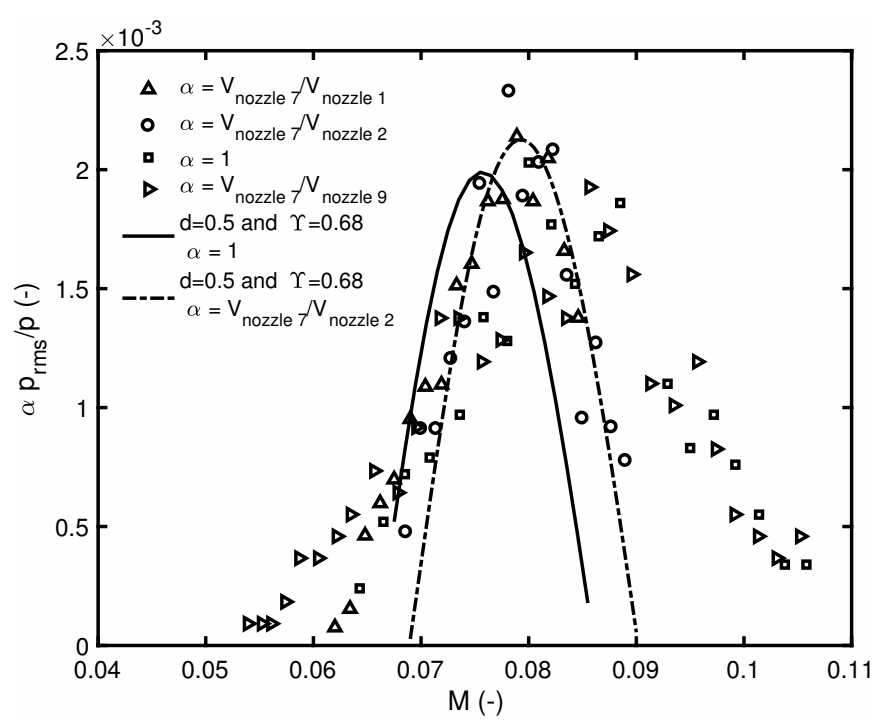

Figure 5.5: Influence of cavity volume $V_{c}$ on the rms pressure as a function of the Mach number for $n=2$.

tude and a rather low Mach number $M_{t h} \simeq 0.116$ compared to the Mach of the measurements, $M_{\mathrm{exp}}$, for the largest amplitudes.

The influence of the pipe length, $L_{p}$, used in the experiments is shown in Table 5.4. The results were obtained using nozzle 7 for a fixed distance between the orifice and the nozzle inlet, $L_{o}=7.1 \times 10^{-2} \mathrm{~m}$, and orifice radius $r_{o}=2.9 \times 10^{-2} \mathrm{~m}$ for which $\Upsilon=0.68$. The Mach numbers observed in the experiments are predicted by the model developed in this work within $20 \%$. For moderate amplitudes, the relative pressure pulsations, $p_{\text {rms }} / p$, are reproduced within a factor two when the factor $K=1 / 3$ is chosen to match the first experiment. When $K$ is varied around this fit value one observes the predicted amplitude to be linearly proportional to $K$. Note that for values of $K$ close or equal to 1 the model can display unphysical behavior, the vortex is not ingested by the nozzle when the circulation becomes too large. This effect is not only observed for a point vortex. It was also observed for a more realistic vorticity distribution by Hulshoff et al. (Hulshoff et al. 2001). Experimental results indicated with an asterisk fall in the so-called low amplitude regime (Bruggeman et al. 1991). The proposed model is not valid in this regime, and drastically overestimates pulsation levels. As indicated earlier, similar behavior is reported by Gallier et al. (Gallier et al. 2009) in detailed numerical flow simulations of hot gas experiments. The slope $((M / f) \mathrm{d} f / \mathrm{d} M)_{\exp }$ determined from the experiments is reproduced within $40 \%$ by the model. The fair prediction of the slope indicates that the losses are well modeled by Eq. (5.18) and the models in appendix $\mathrm{H}$. 


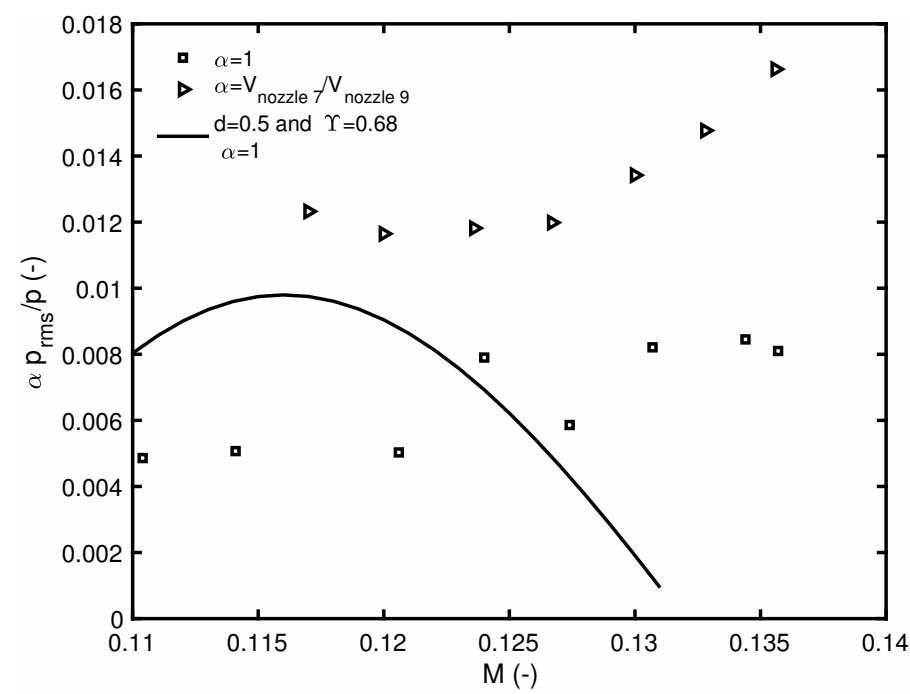

Figure 5.6: Influence of nozzle on the rms pressure as a function of the Mach number for $n=3$.

Table 5.4: Influence of pipe length $L_{p}$.

\begin{tabular}{|c|c|c|c|c|c|c|c|c|}
\hline $\begin{array}{c}\text { Num. } \\
-\end{array}$ & $\begin{array}{l}n \\
-\end{array}$ & $\begin{array}{l}L_{p} \\
\mathrm{~m}\end{array}$ & $\begin{array}{c}M_{\exp } \\
-\times 10^{-2}\end{array}$ & $\begin{array}{l}\left(\frac{p_{\text {rms }}}{p}\right)_{\exp } \\
-\times 10^{-3}\end{array}$ & $\begin{array}{c}\frac{M_{t h}}{M_{\exp }} \\
-\end{array}$ & $\begin{array}{c}\frac{\left(p_{\mathrm{rms}}\right)_{t h}}{\left(p_{\mathrm{rms}}\right)_{\exp }} \\
-\end{array}$ & $\begin{array}{c}\left(\frac{M}{f} \frac{\mathrm{d} f}{\mathrm{~d} M}\right)_{\exp } \\
-\end{array}$ & $\begin{array}{c}\left(\frac{M}{f} \frac{\mathrm{d} f}{\mathrm{~d} M}\right)_{t h} \\
-\end{array}$ \\
\hline 1 & 2 & 0.393 & 8.0 & 2.0 & 0.94 & 1.0 & 0.20 & 0.16 \\
\hline 2 & 3 & 0.393 & 14 & 9.3 & 0.83 & 1.1 & 0.12 & 0.07 \\
\hline 3 & 2 & 0.305 & 11 & 3.7 & 0.88 & 1.1 & 0.17 & 0.14 \\
\hline 4 & 1 & 0.188 & 7.8 & 1.3 & 0.97 & 1.3 & 0.31 & 0.28 \\
\hline $5^{*}$ & 2 & 0.188 & 12 & 4.4 & 1.0 & 4.0 & 0.25 & 0.16 \\
\hline 6 & 1 & 0.163 & 9.8 & 1.6 & 0.88 & 1.8 & 0.39 & 0.30 \\
\hline
\end{tabular}


Table 5.5: Influence of the distance between the orifice and nozzle inlet $L_{o}$. In this table repeating values in columns are replaced by a dashed line.

\begin{tabular}{c|cc|cccc|cc} 
Num. & nozzle & $V_{c}$ & $n$ & $L_{o}$ & $M_{\exp }$ & $\alpha\left(\frac{p_{\mathrm{rms}}}{p}\right)_{\exp }$ & $\frac{M_{t h}}{M_{\exp }}$ & $\frac{\left(p_{\mathrm{rms}}\right)_{t h}}{\left(p_{\mathrm{rms}}\right)_{\exp }}$ \\
- & - & $\mathrm{m}^{3} \times 10^{-5}$ & - & $\mathrm{m} \times 10^{-2}$ & $-\times 10^{-2}$ & $-\times 10^{-3}$ & - & - \\
\hline \hline 18 & 1 & 20.3 & 3 & 4.6 & 7.3 & 2.0 & 0.93 & 0.81 \\
19 & 1 & 20.3 & 2 & 4.6 & 9.0 & 2.8 & 1.1 & 1.1 \\
\hline \hline $7^{*}$ & 7 & 18.4 & 1 & 12.6 & 7.3 & 0.50 & 0.95 & 3.4 \\
1 & $\mid$ & $\mid$ & 2 & 7.1 & 8.0 & 2.0 & 0.94 & 1.0 \\
2 & $\mid$ & $\mid$ & 3 & 7.1 & 14 & 9.3 & 0.83 & 1.1 \\
8 & $\mid$ & $\mid$ & 2 & 4.6 & 13 & 14 & 0.74 & 0.68 \\
9 & 7 & 18.4 & 3 & 4.6 & 8.0 & 1.8 & 0.77 & 1.60 \\
\hline \hline 15 & 9 & 21.8 & 2 & 7.1 & 8.5 & 1.9 & 0.87 & 1.0 \\
16 & 9 & 21.8 & 3 & 7.1 & 14 & 17 & 0.81 & 0.69
\end{tabular}

In Table 5.5, results are shown for cases where the distance between the orifice and the nozzle inlet, $L_{o}$, is varied, and for experiments carried out using nozzles 1, 7 and 9. The pipe length and orifice radius were both fixed at $L_{p}=0.393 \mathrm{~m}$ and $r_{o}=2.9 \times 10^{-2} \mathrm{~m}$, respectively for which $\Upsilon=0.68$. All Mach numbers are reproduced within $25 \%$ using the model described in this study. In column 1 the number of the experiment is shown. For points in the moderate amplitude regime (Bruggeman et al. 1991) the relative pressure pulsations $p_{\mathrm{rms}} / p$ are reproduced within $60 \%$. The low amplitude experiment $7^{*}$ is overestimated by a factor of three, when using the moderate amplitude model.

In Table 5.6, results are shown for various orifice radii, $r_{o}$, and their associated vena contracta factors, $\Upsilon$. Nozzle 7 is used. The pipe length, and orifice length were both fixed at $L_{p}=0.393 \mathrm{~m}$ and $L_{o}=7.1 \times 10^{-2} \mathrm{~m}$, respectively. The measured Mach numbers are reproduced within 30\%. The relative pressure pulsations are reproduced within a factor of two. The parameter $K=1 / 3$ in the model is chosen to match the result of experiment 1 . The variation in vena contracta $\Upsilon$ appears to significantly affect the results.

Table 5.7 is similar to Table 5.6 but for a shorter distance, $L_{o}=4.6 \times 10^{-2} \mathrm{~m}$, between the orifice and the nozzle inlet. The pipe length and orifice length were both fixed at $L_{p}=0.393 \mathrm{~m}$ and $L_{o}=4.6 \times 10^{-2} \mathrm{~m}$, respectively. Most of the experimentally obtained Mach numbers are reproduced within 50\%. The relative pressure pulsations are reproduced within approximately a factor of two. However, measurement 22 deviates by a factor of two in Mach number and more than a factor of two in terms of relative pressure pulsation. Still, the predictions provide the order of magnitude of the pulsation amplitudes. 
Table 5.6: Influence of orifice radius $r_{o}$, for $L_{o}=7.1 \times 10^{-2} \mathrm{~m}$.

\begin{tabular}{c|cc|c|cc|cc} 
Num. & $n$ & $r_{o}$ & $\Upsilon$ & $M_{\exp }$ & $\left(\frac{p_{\mathrm{rms}}}{p}\right)_{\exp }$ & $\frac{M_{t h}}{M_{\exp }}$ & $\frac{\left(p_{\mathrm{rms}}\right)_{t h}}{\left(p_{\mathrm{rms}}\right)_{\exp }}$ \\
- & - & $\mathrm{m} \times 10^{-2}$ & - & $-\times 10^{-2}$ & $-\times 10^{-3}$ & - & - \\
\hline \hline 1 & 2 & 2.9 & 0.68 & 8.0 & 2.0 & 0.94 & 1.0 \\
2 & 3 & 2.9 & 0.68 & 14 & 9.3 & 0.83 & 1.1 \\
10 & 2 & 3.1 & 0.72 & 11 & 3.6 & 0.74 & 0.68 \\
$11^{*}$ & 2 & 3.4 & 0.79 & 6.0 & 0.50 & 0.77 & 1.6 \\
12 & 1 & 3.4 & 0.79 & 14 & 2.3 & 0.65 & 1.4
\end{tabular}

Table 5.7: Influence of orifice radius $r_{o}$, for $L_{o}=4.6 \times 10^{-2} \mathrm{~m}$.

\begin{tabular}{c|cc|c|cc|cc} 
Num. & $n$ & $r_{o}$ & $\Upsilon$ & $M_{\exp }$ & $\left(\frac{p_{\text {rms }}}{p}\right)_{\exp }$ & $\frac{M_{t h}}{M_{\exp }}$ & $\frac{\left(p_{\text {rms }}\right)_{t h}}{\left(p_{\mathrm{rms}}\right)_{\exp }}$ \\
- & - & $\mathrm{m} \times 10^{-2}$ & - & $-\times 10^{-2}$ & $-\times 10^{-3}$ & - & - \\
\hline \hline 20 & 2 & 2.9 & 0.68 & 14 & 9.5 & 0.73 & 0.38 \\
21 & 3 & 2.9 & 0.68 & 7.5 & 4.7 & 0.91 & 0.43 \\
22 & 2 & 3.1 & 0.72 & 5.4 & 1.8 & 2.1 & 2.5 \\
23 & 3 & 3.1 & 0.72 & 10 & 7.2 & 0.74 & 0.32 \\
24 & 2 & 3.4 & 0.79 & 9.0 & 3.8 & 1.4 & 1.8 \\
25 & 3 & 3.4 & 0.79 & 14 & 11 & 1.4 & 1.7
\end{tabular}

\subsubsection{Predictions of the model}

As it has been shown that the model predicts moderate amplitude pulsations in axial injection experiments fairly well, the model is used to investigate the influence of modifications in nozzle geometry. The results are presented in Fig. 5.7. The dotted line is for a contraction with a sharp square edge $(d=0)$ with the source concentrated in a single point at $z=0$. The solid line is for a smooth contraction $\left(d / S_{1}=0.5\right)$, with the source concentrated in a single point at $z=0$. The dashed line is for a smooth contraction for which the source is spread out viz. half the cavity volume is placed at $z=0$ and the other half on a point of the contraction for which $\operatorname{Im}\{z\}=0.25 S_{1}$. Spreading out the cavity volume between these two points simulates the finite size of the opening of the nozzle cavity. The Mach number of the predicted maximum pressure pulsation for the sharp contraction is displaced approximately $5 \%$ to the left with respect to the smoother contractions. There is, however, no appreciable impact on the predicted pressure pulsation amplitude for all the geometric variations. This leads one to conclude that the exact geometry of the nozzle does not have a major influence on the generation of pressure pulsations in SRMs.

The model also allows for comparison between the sound produced without cavity, $V_{c}=0$, and sound produced when a nozzle is cavity present. This is illustrated in Fig. 5.8. One observes that the interaction of the vortex with the 


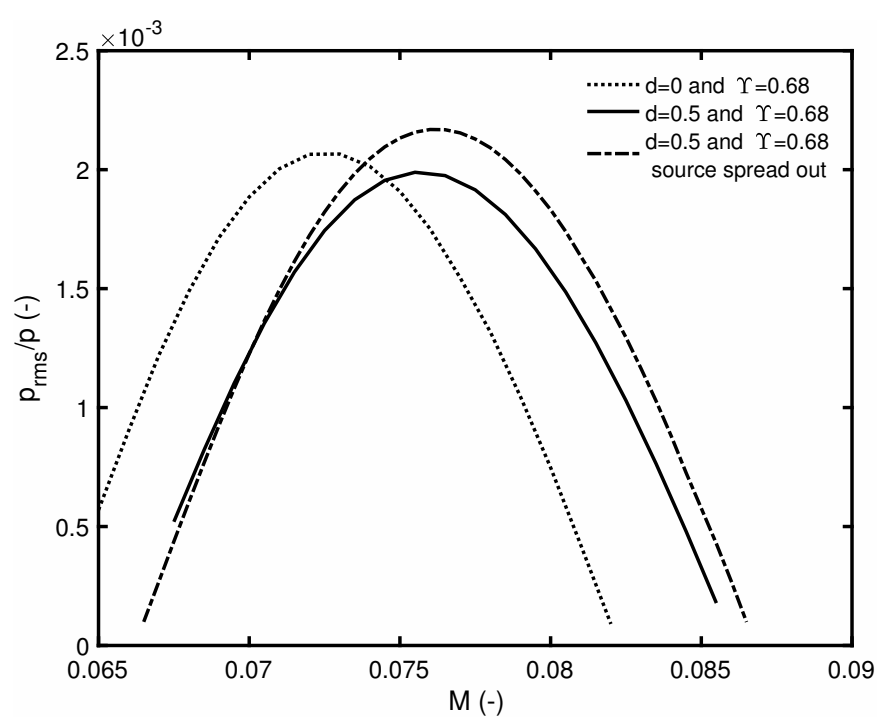

Figure 5.7: Influence of geometric parameters of the nozzle on model predictions.

cavity produces a response two orders of magnitude greater than produced by the ingestion of the vortex by the nozzle. Here the residual compressibility effect of the fluid around the nozzle inlet described in chapter 4 (section 4.9) was not considered. If it had been, the difference between model predictions for the case with and without nozzle cavity, would have been smaller. One would have to replace $V_{c}$ in the present chapter by $V_{\text {eff }}=V_{c}+V_{0}$ as discussed in chapter 5 . Note that experiments carried out by Anthoine (Anthoine 2000) with $V_{c}=0$ did not show significant pulsation amplitudes, as such the pulsations are in the low amplitude regime and cannot be predicted by the present model.

\subsection{Conclusion}

An analytical model based on an energy balance has been proposed to predict the amplitudes of pulsations in a cold gas model of a SRM with axial flow injection and vortex shedding at a thermal inhibitor. The acoustic energy losses are described by linear quasi-steady models for acoustic radiation at the nozzle, vortex shedding at the inhibitor and viscous dissipation in the porous wall used for injection (appendix H). The energy balance model predicts losses increasing quadratically with the pulsation amplitude. These losses are balanced by the sound production due to the interaction of vortices shed at the inhibitor with the critical nozzle at the downstream end of the SRM.

The magnitude of the circulation of the vortices, $\Gamma$, is estimated as a fraction $K$ of the vorticity shed within one period of oscillation concentrated in a line 


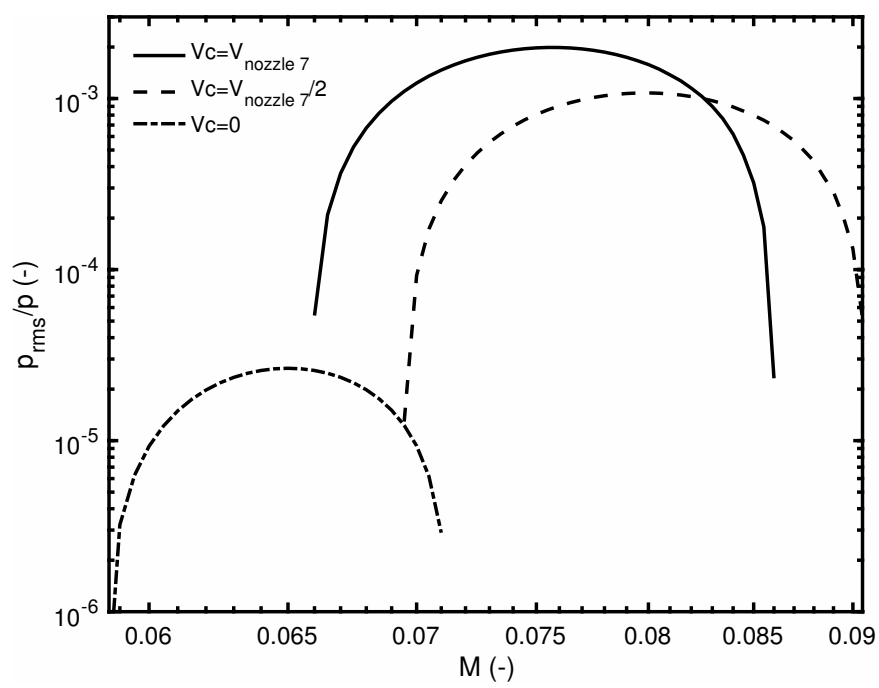

Figure 5.8: Influence of the cavity volume $V_{c}$.

vortex. The acoustic mode number, $n$, is a user provided input to the energy balance model. The oscillation frequency, $f$, is assumed to be equal to the corresponding eigenfrequency, $f_{n}$.

The factor $K=1 / 3$ is fitted to one of the experimental results and it is assumed to account for the effect of turbulence in the flow, as well as other differences between the model and the actual flow. This is in accord with previous numerical simulations, which indicate that including turbulence reduces the predicted pulsations by about a factor two, compared to a laminar flow simulation.

The dynamics of the vortex is described by a planar incompressible potential flow model. It is assumed that the acoustic field only determines the moment (phase) of vortex shedding at the inhibitor. The vortex circulation and path are independent of the amplitude of pulsation. The acoustic power generated by the vortex is calculated using Howe's energy corollary. This power scales linearly with the pulsation amplitude. This allows for a prediction of the pulsation amplitude for moderate acoustic velocity amplitudes $10^{-2} \leq\left|u^{\prime}\right| / U \leq 10^{-1}$.

At lower acoustic velocity amplitudes $\left|u^{\prime}\right| / U<10^{-2}$, the energy balance model severely overestimates the pulsation level. Predicting low amplitude pulsations appears to be difficult even when using sophisticated numerical models (Gallier et al. 2009). At low amplitudes, the vortex circulation becomes amplitudedependent. Furthermore, if the linear regime is approached, the power predicted by Howe's corollary scales quadratically with the amplitude. Hence, the balance between acoustic losses and vortex sound generation does not allow accurate amplitude prediction. A minor increase in losses can reduce predicted 
pulsation levels by orders of magnitude.

In the moderate amplitude regime, the energy balance model predicts data of Anthoine et al. (Anthoine 2000; Yildiz 2000) quite well. The pulsation amplitudes are predicted within a factor two and the Mach number for the maximum pulsation level within $20 \%$ for orifice position $L_{o} / r_{p}=1.9$ and orifice radius $r_{o}=2.9 \times 10^{-2} \mathrm{~m}$. For $L_{o} / r_{p}=1.2$ and orifice radius $r_{o}=2.9 \times 10^{-2} \mathrm{~m}$, the model predicts the pulsation levels within a factor 3 and the Mach number within a factor two.

The influence of the orifice radius, $r_{o}$, has been investigated. The predictions agree with Anthoine (Anthoine 2000) and Yildiz (Yildiz 2000). The exception is measurement 22, for which the prediction deviates by a factor two in Mach number and more than a factor two in terms of relative pulsation amplitude. Even in that case, the energy balance model provides the order of magnitude of the pulsation amplitudes.

By applying the theory of Culick (Culick 2006) to the vortex sound analogy of Howe (Howe 1998; Howe 2007) one can obtain the Mach number dependence of the oscillation frequency around a pulsation maximum. The slope $(M / f) \mathrm{d} f / \mathrm{d} M$ is determined by the losses. Comparison between theory and experiments indicates that larger deviations are due to inaccuracies in the source model, in particular the description of the vortex flow, rather than inaccuracies in the losses.

An important result (Anthoine 2000) is that the pulsation amplitude is proportional to the volume $V_{c}$ of the cavity around the inlet of the integrated nozzle. The energy balance model confirms this and predicts that pulsation amplitudes generated by the ingestion of the vortex are two order of magnitude lower than those generated by the interaction of the nozzle with the cavity. Here the model could be improved by replacing $V_{c}$ by $V_{\text {eff }}=V_{c}+V_{0}$ is introduced in chapter 4 .

The energy balance model also indicates that only minor differences result due the use of nozzle with a sharp inlet compared to a rounded inlet. A variation in the position of the nozzle cavity inlet has only a small effect on the pulsation amplitude. This illustrates that the model provides insight in the influence of geometric parameters on the pulsation levels.

A correction to the energy balance model taking into account the axis-symmetrical geometry of the vortices and the effect of turbulence should be considered as a next step. This would involve sophisticated numerical simulations. Although the present model is crude, relative to numerical simulations, it is almost completely analytical and well suited for parametric analysis. 


\title{
Chapter 6
}

\section{Entropy indirect noise compared to vortex driven indirect noise in large solid rocket motors: a numerical investigation of the influence of a nozzle cavity}

\begin{abstract}
Numerical simulations based on a two-dimensional plane frictionless compressible flow model are used to study the effect of the cavity surrounding the inlet of an integrated nozzle on indirect noise radiated upstream. This indirect noise provides a feedback mechanism which can induce detrimental self-sustained pressure pulsations in Solid Rocket Motors (SRMs). Two types of indirect noise sources are considered: the interaction of the nozzle with vortices generated by hydrodynamic instabilities triggered by the acoustic oscillation, and the ingestion by the nozzle of entropy spots resulting from modulation of the combustion process. A novel scaling law for entropy-spot-nozzle interaction is proposed. Published cold-gas scale model experiments have demonstrated that vortex-nozzle interaction noise is proportional to the volume of the cavity around the integrated nozzle inlet of a SRM. Hot-gas-scale models with combustion have also demonstrated that the nozzle cavity volume is crucial. The following question arises: what is the contribution by entropy-spot-nozzle interaction to self-sustained pressure pulsations in SRMs? Evidence is provided that entropy noise generation is marginally affected by the nozzle cavity. From this it is inferred that vortex noise likely dominates the establishment of self-sustained pressure pulsations in scale experiments of large SRMs with combustion.
\end{abstract}


Chapter 6 - Entropy indirect noise Compared to VORTEX Driven 138 INDIRECT NOISE IN LARGE SOLID ROCKET MOTORS: A NUMERICAL INVESTIGATION OF THE INFLUENCE OF A NOZZLE CAVITY

\subsection{Introduction}

Indirect sound sources can lead to the establishment of a feedback loop in a combustion chamber leading to self-sustained pressure pulsations (Morgans and Duran 2016; Anthoine 2000; Anthoine et al. 2002; Gallier et al. 2009). This is true for both entropy-spot and vortex-driven sound sources. An entropy spot is a density inhomogeneity in a flow due to unsteady heat release or non-uniform combustion. It produces sound when accelerated through the nozzle while leaving the combustion chamber (Morgans and Duran 2016; Howe 1975; Tao et al. 2017). This process will be referred to here as entropy-spot-nozzle interaction. Vortex-driven indirect combustion noise is generated when a vortex (vorticity patch), shed upstream, makes its way out of the combustion chamber. This process will be referred to as vortex-nozzle interaction (Hulshoff et al. 2001; Hirschberg et al. 2017).

In both cases the resulting acoustic wave travels upstream, where it maintains an acoustic standing wave, which in turn causes the generation of new entropy spots or vortices (i.e. a feedback loop is established). The presence of a cavity around the nozzle inlet (as in integrated nozzles) has been demonstrated to have a major influence on vortex-driven self-sustained pressure pulsations in Solid Rocket Motors (SRMs) (Anthoine 2000; Anthoine et al. 2002; Gallier et al. 2009; Hirschberg et al. 2018). Indeed, cold-gas experiments using a scale model of the Ariane 5 SRM show that the limit-cycle amplitude of vortex-driven self-sustained pressure pulsations is proportional to the nozzle cavity volume $V_{c}$ (Anthoine et al. 2002). The role and importance of this cavity volume $V_{c}$ for vortex-driven pulsations has been analyzed in chapters 4 and 5 .

In this chapter, the influence of a nozzle cavity surrounding the nozzle inlet on upstream indirect entropy noise radiation is reported for the first time. These results are used to provide a new interpretation the of results of the hot-gasscale experiments (involving combustion of non-metalized propellant), reported by Gallier et al. (Gallier et al. 2009).

In the existing literature, the typical length scale of an entropy spot is often assumed to be the channel height, $S_{1}$. The interaction with the nozzle is often described by assuming a plane entropy wave (Marble and Candel 1977). In contrast with these studies, circular entropy spots will be considered here, with a radius smaller than the channel height. The idea is that in this case the theory of Ffowcs Williams-Howe (Ffowcs Williams and Howe 1975) for small entropy spots can be used.

In section 6.2 a brief description of the computational approach is provided. In order to assess the sensitivity of the results the problem parameters are varied within the range expected in SRMs. As a by product, this study also 
Chapter 6 - Entropy indirect nOISE COMPARED TO VORTEX DRIVEN INDIRECT NOISE IN LARGE SOLID ROCKET MOTORS: A NUMERICAL INVESTIGATION 139 OF THE INFLUENCE OF A NOZZLE CAVITY

establishes dimensionless representations, allowing an efficient representation of the data. For the acoustic pulse produced by vortex-nozzle interactions, scaling laws were already established in chapter 4 (Hirschberg et al. 2018). For entropy-nozzle interaction, a scaling law will be proposed. The influence of the upstream Mach number on the acoustic pulse produced by entropy spot interaction is described given in section 6.3. In section 6.4, the influence of the core radius of the entropy spot is described. The influence of the upstream release height is discussed in section 6.5. In section 6.6, an original interpretation of hot-gas-scale experiments reported by Gallier et al. (Gallier et al. 2009) is provided.

\subsection{Description of the computational approach}

Systematic studies of vortex-nozzle and entropy-spot-nozzle interaction have been carried out for the present study using the two-dimensional plane Euler Internal Aeroacoustics code (EIA) described by Hulshoff et al. (Hulshoff et al. 2001; Hulshoff 2016b) (see appendix I for a description based on the EIA manual (Hulshoff 2016b)). Vortex cores with radius $R_{\Gamma}$ and circulation $\tilde{\Gamma} \equiv \Gamma /\left(S_{1} U\right)$ (chapter 4 ) or a circular entropy spot of radius $R_{s}$ and maximum density difference $\delta \rho$ with respect to their surroundings are generated upstream from a critical nozzle. These structures travel downstream exiting through the nozzle and resulting in an upstream traveling acoustic pulse.

The two nozzle inlet geometries, as sketched in Fig. 6.1, were considered. Fig. 6.1(a) shows the integrated nozzle geometry with a cavity surrounding its inlet, and Fig. 6.1(b) shows the nozzle with the inlet forming a right angle corner with the combustion chamber side wall. The volume $V_{c}$ chosen in the simulations corresponds to the volume of an upstream duct segment of length $0.7 S_{1}$, in this two-dimensional plane geometry. This corresponds to nozzle 2 in the cold-gas experiments of Anthoine (Anthoine 2000; Hirschberg et al. 2018) and is, at a reduced scale, representative of the nozzle cavity volume of a Ariane 5 SRM (Anthoine 2000).

For the EIA simulations, a second-order total-variation-diminishing scheme with Roe's approximate Riemann solver was used in combination with a five stage Runge-Kutta time marching method (Venkatakrishnan and Jameson 1988; Hulshoff et al. 2001). Convergence tests for the reported results demonstrated an observed order of accuracy of 1.8 with an associated discretization error of approximately $1 \%$. A pressure probe was placed at a distance $6 S_{1}$ upstream from the nozzle inlet and used to record the resulting upstream traveling acoustic wave $\left(S_{1}\right.$ is half the combustion chamber height, measured from the outer wall to the symmetry axis upstream from the nozzle inlet see Fig. 6.1(a)). The 
Chapter 6 - Entropy indirect noise Compared to VOrTex driven 140 INDIRECT NOISE IN LARGE SOLID ROCKET MOTORS: A NUMERICAL INVESTIGATION OF THE INFLUENCE OF A NOZZLE CAVITY

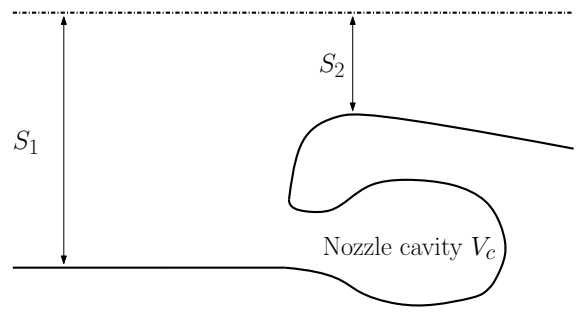

(a) Integrated nozzle

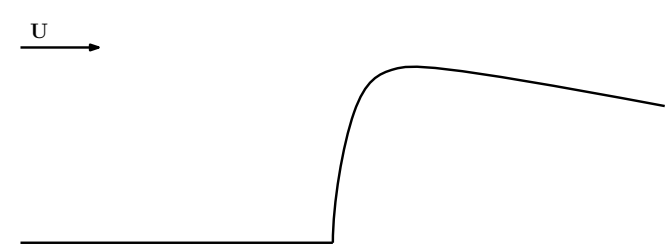

(b) Right angle inlet nozzle

Figure 6.1: Nozzle inlet geometries considered respectively with nozzle cavity 6.1(a) and with a right angle nozzle inlet 6.1(b). For both $S_{1}$ is the upstream channel height and $S_{2}$ is the height in the throat, both measured with respect to the symmetry line (dash dotted).

upstream boundary condition was anechoic (non-reflecting for acoustic waves), corresponding to a semi-infinite upstream duct.

\subsection{Influence of upstream Mach number on upstream acoustic response}

To investigate the influence of the upstream Mach number $M$ on the acoustic response due to entropy-spot-nozzle interaction, simulations with $M=0.053$, 0.097 and 0.197 have been carried out, both with (Fig. 6.1(a)) and without (Fig. 6.1(b)) the presence of a nozzle cavity volume. Entropy spots were considered with maximum relative density differences $\delta \rho / \rho=-0.03,-0.06$ and -0.12 for $M=0.053,0.097$ and 0.197 , respectively.

In cold-gas scale experiments, there are naturally occurring entropy differences carried by vortices. These are of the order $(\gamma-1) M^{2} / 2 \simeq 0.2 M^{2}$. Thus, taking the upper bound for mach number as $M \simeq 0.2, \delta \rho / \rho \simeq 0.01$ was considered as the lower bound for the relative density variation. Very large density variations (of order unity or larger) are not considered, as these are expected not to follow the base flow, hindering a potential comparison with simplified models which consider the acceleration of a an entropy spot going to through the contraction to be equal to the stationary base flow acceleration (Ffowcs Williams and Howe 1975). Furthermore, as typical pressure fluctuations in large SRMs or three orders of magnitude lower than the static pressure in the combustion chamber, one does not expect large relative density fluctuations.

The results of the entropy-spot-nozzle interaction simulations are shown in Fig. 6.2. There the scaled acoustic response $S_{1} p^{\prime} /\left(R_{s} c^{2} M \delta \rho\right)^{1}$ is shown as a func-

\footnotetext{
${ }^{1}$ the $R_{s} / S_{1}$ scaling factor is discussed in section 6.4
} 
Chapter 6 - Entropy indirect noise COMPAREd to Vortex DRIVEN INDIRECT NOISE IN LARGE SOLID ROCKET MOTORS: A NUMERICAL INVESTIGATION 141 OF THE INFLUENCE OF A NOZZLE CAVITY

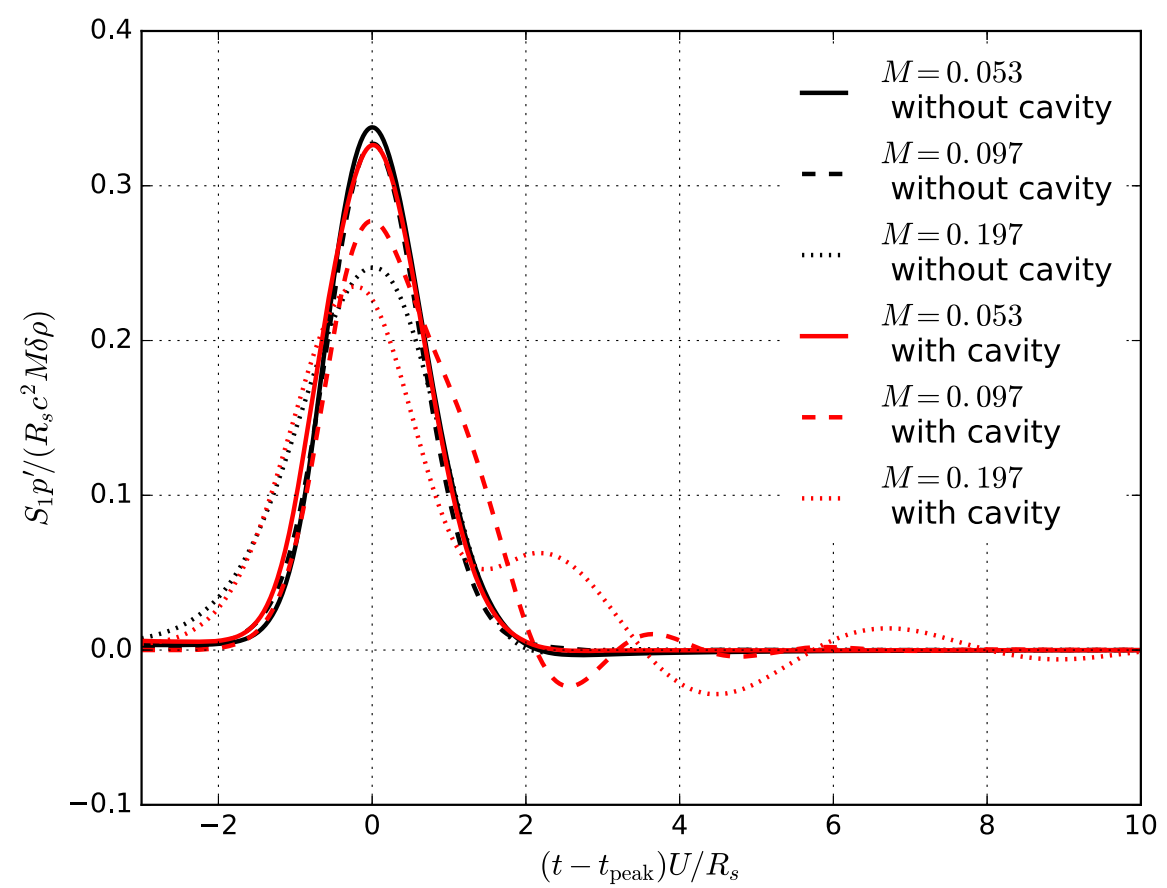

Figure 6.2: Influence upstream Mach number $M$ on entropy-spot-nozzle interaction with and without nozzle cavity. The scaled acoustic response $S_{1} p^{\prime} /\left(R_{s} c^{2} M \delta \rho\right)$ is shown as a function of the dimensionless time $t U / R_{s}$ for upstream Mach numbers $M=0.053$, 0.097 and 0.197. Results obtained with nozzle cavity are shown in red, those obtained without nozzle cavity are shown in black. The relative maximum density difference with respect to its surroundings of the entropy spots was $\delta \rho / \rho=-0.03,-0.06$ and -0.12 for upstream Mach numbers $M=0.053$, 0.097 and 0.197 respectively. The upstream release height $h$ and the entropy-spot-core radius $R_{s}$ were fixed at $h=0.4 S_{1}$ and $R_{s}=0.3 S_{1}$. 
Chapter 6 - Entropy indirect noise COMPAREd TO VORTEX Driven 142 INDIRECT NOISE IN LARGE SOLID ROCKET MOTORS: A NUMERICAL INVESTIGATION OF THE INFLUENCE OF A NOZZLE CAVITY

tion of $\left(t-t_{\text {peak }}\right) U / R_{s}$. The time axes has been scaled by the entropy-spot-core radius, $R_{s}$, for reasons that will be made clear in section 6.4. Due to the difference in upstream Mach number, $M$, the pulses occur at different points in dimensionless time $U t / R_{s}$. To compare the pulses directly, the results are shifted by dimensionless time $U t_{\text {peak }} / R_{s}$ where $t_{\text {peak }}$ is the time at the maximum of the pulse amplitude. The results of simulations without nozzle cavity are shown in black, while the results obtained with the presence of a nozzle cavity are shown in red.

For Mach numbers $M=0.097$ and 0.197 , note the pronounced presence of the second cavity-channel transversal mode as observed for vortex-nozzle interaction (section 4.7). This deforms the main pulse (e.g. for $M=0.097$ the downward going slope on $\left.0<\left(t-t_{\text {peak }}\right) U / R_{s}<2\right)$. The presence of a cavity appears to slightly reduce the pulse amplitude. This reduction is more pronounced for $M=0.097$ and 0.197 , compared with the $M=0.053$ case. For these two higher Mach numbers, the transverse mode oscillation is strong. Hence, one suspects the peak reduction to be a consequence of the transverse oscillation. From the data of Fig. 6.2 one concludes that the nozzle cavity does not enhance sound radiation during entropy spot nozzle interaction.

Both in the presence and in absence of a nozzle cavity volume, a linear dependence on upstream Mach number $M$ of $S_{1} p^{\prime} /\left(R_{s} c^{2} \delta \rho\right)$ due to entropy-spotnozzle interaction has been found. It is interesting that the entropy-spot-nozzle interaction pressure pulse scales with the static pressure difference $\delta p \sim c^{2} \delta \rho$, rather than the dynamic pressure $\left(\frac{1}{2} \rho U^{2}\right)$, used to scale the pulse due to vortexnozzle interaction (chapter 4).

\subsection{Influence of core radius on upstream acoustic re- sponse}

Simulations have been carried out to investigate the influence of the entropy spot-core radius $R_{s}$, on the acoustic response due to entropy-spot-nozzle interaction. These were performed for nozzles both with and without a cavity. The upstream Mach number was fixed at $M=0.053$. The entropy spots were generated upstream from the contraction and released at height $h=0.4 S_{1}$ from the channel wall. Results are shown in Fig. 6.3 for entropy spot-core radii $R_{s}=0.2 S_{1}, 0.3 S_{1}$ and $0.4 S_{1}$. Those for a nozzle with a cavity volume are shown in red, and those for a nozzle without cavity are shown in black. The dimensionless acoustic response $S_{1} p^{\prime} /\left(R_{s} M c^{2} \delta \rho\right)$ is shown as a function of dimensionless time $\left(t-t_{\text {peak }}\right) U / R_{s}$ where $U$ is the average upstream velocity in the axial direction and $R_{s}$ is the entropy-spot-core radius.

The upstream acoustic response due to entropy-spot-nozzle interaction was 
Chapter 6 - Entropy indirect noise compared to Vortex DRIVEN INDIRECT NOISE IN LARGE SOLID ROCKET MOTORS: A NUMERICAL INVESTIGATION 143 OF THE INFLUENCE OF A NOZZLE CAVITY

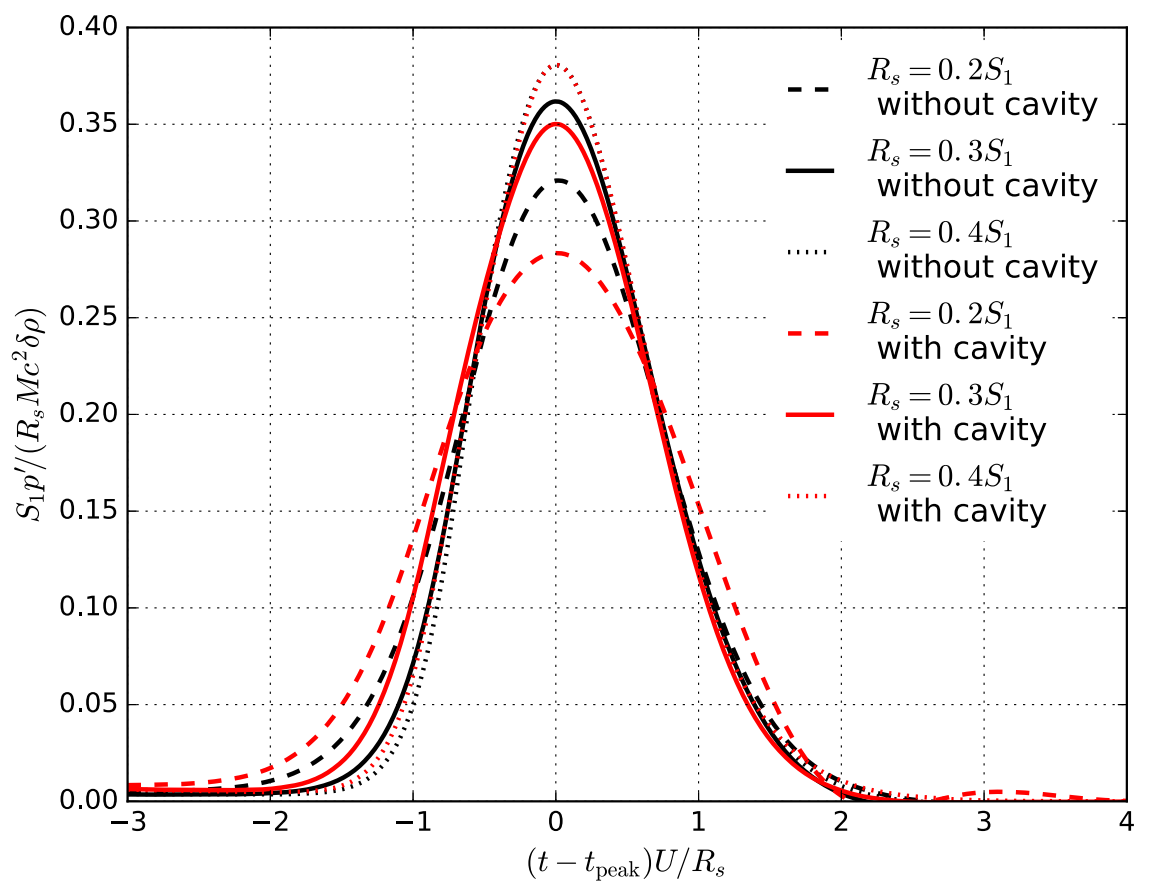

Figure 6.3: Influence of the entropy-spot-core radius on upstream acoustic response: entropy-spot-nozzle interaction with and without nozzle cavity. The scaled acoustic response $S_{1} p^{\prime} /\left(R_{s} c^{2} M \delta \rho\right)$ is shown as a function of the dimensionless time $t U / R_{s}$ for entropy-spot-core radii $R_{s}=0.2,0.3$ and 0.4 . Results obtained without the presence of a nozzle cavity are shown in black, while results obtained with the presence of a nozzle cavity are shown in red. The upstream Mach number $M$ and upstream release height were fixed at $M=0.053$ and $h=0.4 S_{1}$ respectively. 
Chapter 6 - Entropy indirect noise COMPAREd TO VORTEX Driven 144 INDIRECT NOISE IN LARGE SOLID ROCKET MOTORS: A NUMERICAL INVESTIGATION OF THE INFLUENCE OF A NOZZLE CAVITY

found to scale linearly with entropy-spot-core radius $R_{s}$. As can be expected the pulse time length is proportional to $R_{s}$, and for the two-dimensional plane model discussed here $\delta m_{s}$, scales with $R_{s}^{2}$. This means that time integral $\int p^{\prime} \mathrm{d} t$ divided by the mass of the entropy spot $\delta m_{s}$ is independent of the entropy-spot-core radius $R_{s}$ (viz., $\int p^{\prime} \mathrm{d} t / \delta m_{s}$ is independent of $R_{s}$ ). One has $\int p^{\prime} \mathrm{d} t \sim p^{\prime} R_{s} / U$ and $\delta m_{s} \sim R_{s}^{2}$. From this one finds that $p^{\prime} \sim R_{s}$. The physical explanation for this scaling behavior remains to be elucidated.

In Fig. 6.3 one observes, that the presence of a nozzle cavity has little influence on the dimensionless upstream acoustic response $S_{1} p^{\prime} /\left(R_{s} M c^{2} \delta \rho\right)$. Indeed, the largest deviation of approximately $13 \%$ between peak values for simulations with and without the presence of a nozzle cavity occurs for $R_{s}=0.2 S_{1}$. This result will be used later.

\subsection{Influence of release height on upstream acoustic response}

An investigation of entropy-spot-nozzle interaction simulations with varied upstream release heights has been carried out. The simulations were done with fixed entropy-spot-core radius $R_{s}=0.3 S_{1}$ and upstream Mach number $M=$ 0.053, for both nozzle inlet geometries Fig. 6.1 (i.e. with and without a nozzle cavity).

Results for upstream release heights of $h=0.4 S_{1}, 0.5 S_{1}$ and $0.6 S_{1}$ are shown in Fig. 6.4. The red lines are for simulations for a nozzle with a cavity and the black lines are for simulations without a nozzle cavity. One observes that the upstream release height has a some influence on the dimensionless upstream acoustic response $S_{1} p^{\prime} /\left(R_{s} M c^{2} \delta \rho\right)$. Between the results obtained with release heights $h=0.4 S_{1}$ and $0.6 S_{1}$, there are deviations of approximately $18 \%$ (nozzle without cavity) and $15 \%$ (nozzle with cavity).

However, one observes that the presence of a nozzle cavity does not significantly influence the dimensionless upstream acoustic response $S_{1} p^{\prime} /\left(R_{s} M c^{2} \delta \rho\right)$. Indeed for the upstream release height $h=0.6 S_{1}$, the largest deviation observed between simulation with and without cavity is approximately $7 \%$. 
Chapter 6 - Entropy indirect noise COMpared to Vortex DRIVEN INDIRECT NOISE IN LARGE SOLID ROCKET MOTORS: A NUMERICAL INVESTIGATION 145 OF THE INFLUENCE OF A NOZZLE CAVITY

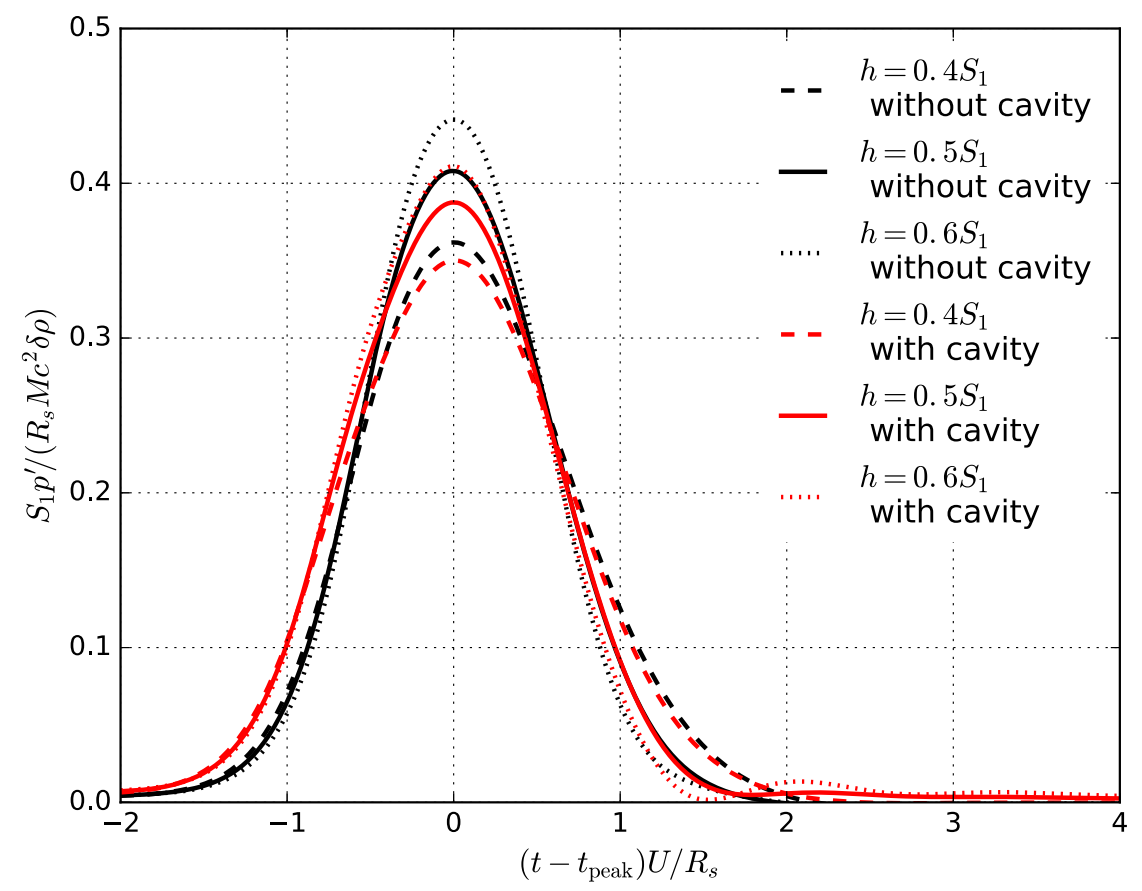

Figure 6.4: Influence of the upstream release height on entropy-spot-nozzle interaction acoustic response: with and without nozzle cavity. The scaled acoustic response $S_{1} p^{\prime} /\left(R_{s} c^{2} M \delta \rho\right)$ is shown as a function of the dimensionless time $t U / R_{\text {s }}$ for upstream release heights $h / S_{1}=0.4,0.5$ and 0.6. Results obtained without the presence of a nozzle cavity are shown in black, while results obtained with the presence of a nozzle cavity are shown in red. The upstream Mach number $M$ and entropy-spot-core radius $R_{s}$ were fixed at $M=0.053$ and $h=0.4 S_{1}$ respectively. 
Chapter 6 - Entropy indirect noise compared to Vortex DRIVEn

146 INDIRECT NOISE IN LARGE SOLID ROCKET MOTORS: A NUMERICAL INVESTIGATION OF THE INFLUENCE OF A NOZZLE CAVITY

\subsection{Comparison of the effect of a nozzle cavity vol- ume on vortex-nozzle and entropy-spot-nozzle in- duced indirect noise}

Until now, the focus of the discussion was on the establishment of scaling laws. In this section, simulation results are used to discuss the role of the integrated nozzle (nozzle with cavity Fig. 6.1(a)) on upstream-traveling indirect sound due to entropy-spot-nozzle and vortex-nozzle interactions.

Fig. 6.5 compares the results of the entropy-spot-nozzle interaction simulations (Fig. 6.5(a)) and vortex-nozzle interaction simulations (Fig. 6.5(b)) of upstream Mach number $M \equiv U / c=0.053$ and 0.058 , respectively. The choice of the low Mach number reduces transverse channel-cavity mode oscillations. The entropy-spot-nozzle interaction simulations were carried out with $\delta \rho / \rho=-0.03$, while for the vortex-nozzle interaction simulations a dimensionless vortex circulation of $\tilde{\Gamma} \equiv \Gamma /\left(U S_{1}\right)=-0.9$ was used.

The entropy-spot-nozzle and vortex-nozzle interaction obey different scaling rules, as described in the previous sections for entropy-spot-nozzle interaction and chapter 4 for vortex-nozzle interaction. On the axes of Fig. 6.5(a) and Fig. 6.5(b) the same dimensionless quantities are shown. Namely, $p^{\prime} /\left(\rho U^{2}\right)$ is shown as a function of $t U / S_{1}$.

It is striking that the entropy-spot-nozzle interaction is only marginally affected by the presence of the cavity. This was found to be true for a range of values of the density inhomogeneity $0.03 \leq \delta \rho / \rho \leq 0.12$ (section 6.3), higher upstream Mach numbers $0.05 \leq M \leq 0.20$ (section 6.3), entropy-spot-core radii $0.2 \leq R_{s} / S_{1} \leq 0.4$ (section 6.4) and upstream release heights $0.3 \leq h / S_{1} \leq 0.5$ (section 6.5). In all cases, the deviations of dimensionless peak heights were less than 20\%. In contrast, one observes in Fig. 6.5(b) that the vortex-nozzle interaction pulse amplitude is increased by a factor three by the presence of the nozzle cavity. Furthermore, the shape of the pulse is also drastically modified. The strong influence due to the presence of a nozzle cavity on vortex-nozzle interaction induced upstream traveling sound persists, for different values of upstream Mach number $M$ (with cavity Fig. 4.22 and without cavity Fig. 4.28) vortex-core radius $R_{\Gamma}$ (with cavity Fig. 4.17 and without cavity Fig. 4.25 ) and upstream release height $h$ (with cavity Fig. 4.18 and without cavity Fig. 4.25).

For both cases the sound source can, using the aeroacoustic analogy (Howe 1975), be qualitatively understood as a force density field $\mathbf{f}$ acting on a reference potential flow i.e. the acoustic field. In the case of vortex sound, this force field is $\mathbf{f}_{v}=-\rho(\boldsymbol{\omega} \times \mathbf{v})$ where $\rho$ is the fluid density, $\mathbf{v}$ is the local flow velocity 
Chapter 6 - Entropy indirect noise compared to Vortex driven INDIRECT NOISE IN LARGE SOLID ROCKET MOTORS: A NUMERICAL INVESTIGATION 147 OF THE INFLUENCE OF A NOZZLE CAVITY

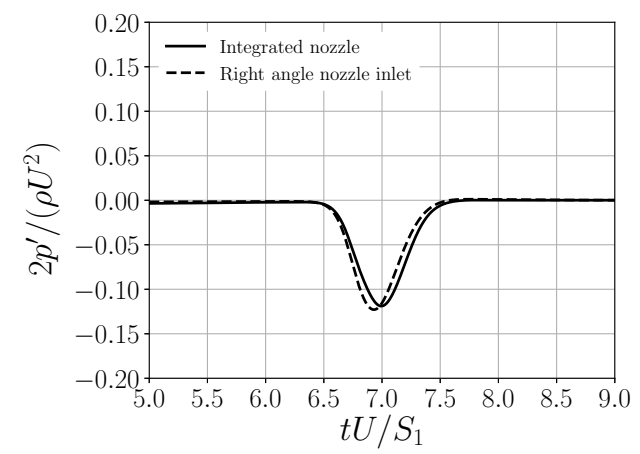

(a) entropy-spot-nozzle interaction

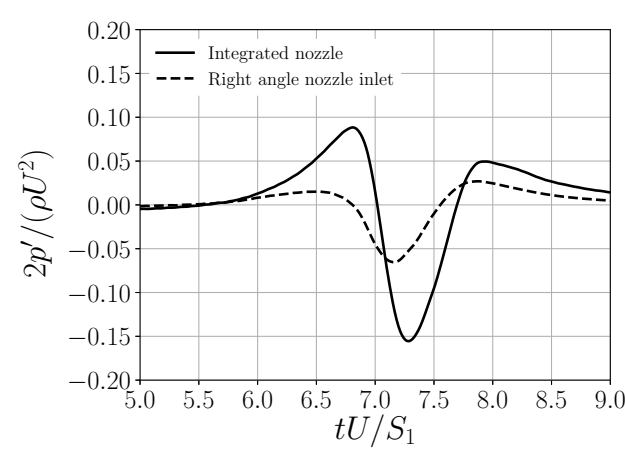

(b) Vortex-nozzle interaction

Figure 6.5: Upstream acoustic response, for both a right angle nozzle inlet geometry and an integrated nozzle, due to entropy-spot-nozzle 6.5(a) and vortex-nozzle interaction $6.5(b)$ respectively.

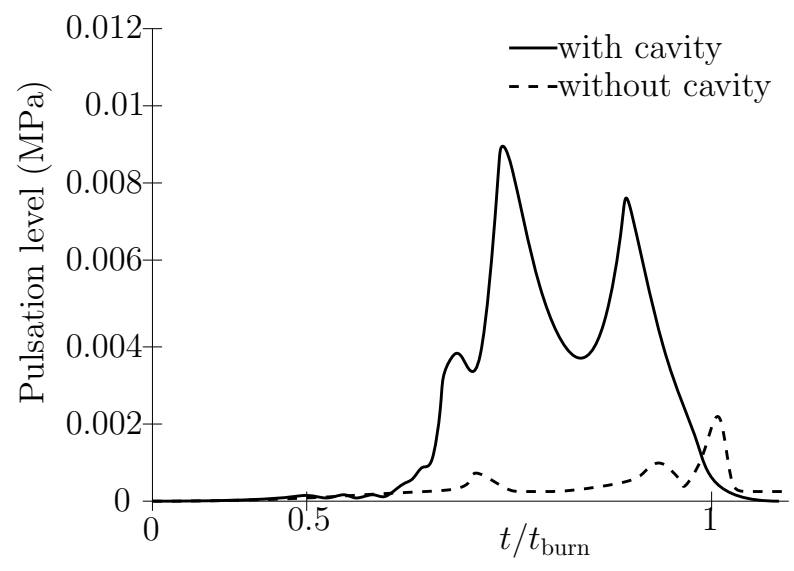

Figure 6.6: LP9 scale experiments with combustion, with and without a nozzle cavity after (Gallier et al. 2009). Here $t_{\mathrm{burn}}$ is the duration of a firing i.e. $t / t_{\mathrm{burn}}=1$ is when all the fuel has been depleted. 
Chapter 6 - Entropy indirect noise COMPAREd TO VORTEX Driven 148 INDIRECT NOISE IN LARGE SOLID ROCKET MOTORS: A NUMERICAL INVESTIGATION OF THE INFLUENCE OF A NOZZLE CAVITY

and $\boldsymbol{\omega} \equiv \nabla \times \mathbf{v}$ is the vorticity. In the case of entropy sound, this force is $\mathbf{f}_{s}=\delta \rho \mathrm{Dv} / \mathrm{D} t$ (Howe 1975), where $\delta \rho$ is the density difference between the local density in the entropy spot and the density of the surrounding homentropic flow. Let $G$ designate the acoustic response due to a point source generated pulse i.e. $G$ is the Green's function. The gradient of the Green's function $G$, $\nabla G$ has two components. The first, $\nabla G_{r}$, is the acoustic flow due to radiation through the nozzle, which in first approximation is parallel to the flow velocity $\mathbf{v}$ along the path of the vortex. The latter is close to a particle path. The second component, $\nabla G_{c}$, is the acoustic flow generated by compression of the fluid around the nozzle inlet. This cavity induced acoustic flow, $\nabla G_{c}$, is in first order approximation normal to $\mathbf{v}$ along the path of the vortex and entropy spot (Anthoine et al. 2002; Hirschberg et al. 2018). Consequently $\mathbf{f}_{v}$ mostly radiates noise thanks to the enhanced compressibility component $\nabla G_{c}$ due to the presence of nozzle cavity. This effect appears to be important, as shown in Fig. 6.5(b). As explained in chapter 4, the cavity induces a delayed reflection of the sound pulse in addition to the initially radiated pulse. This results in an acoustic response which is the time derivative of the original pulse (induced by $\mathbf{f}_{v}$ ). The normal component of $\mathbf{f}_{s}$ aligned with $\nabla G_{c}$ is due to centrifugal acceleration. This effect appears to be negligible, as observed in Fig. 6.5(a). The added compressibility due to the nozzle cavity does not significantly affect the acoustic radiation caused by the entropy spot. This indicates that the acceleration normal to the flow (centrifugal acceleration) is negligible. This might be due to the fact that the particle path displays an inflection point as the fluid enters the nozzle.

It is striking that in the LP9 hot-gas-scale experiments (with combustion of non-metalized propellant) reported by Gallier et al. (Gallier et al. 2009) (Fig. 6.6), the self-sustained pressure pulsation amplitude increases by an order of magnitude in the presence of a nozzle cavity. Combined with the current observations, this, suggests that indirect sound sources are dominated by vortexnozzle interaction. Furthermore, one expects that direct combustion noise is not affected by the relatively small nozzle cavity. Therefore, one concludes that direct noise is not dominant in these hot-gas-scale experiments.

\subsection{Conclusion}

Results have been presented which suggest that in contrast to vortex-nozzle interaction, entropy-spot-nozzle interaction is not significantly influenced by the presence of a nozzle cavity.

Systematic parameter studies varying the upstream Mach number $M$ and entropyspot-core radius $R_{s}$ have led to the discovery of a new scaling rule. The acoustic response due to entropy-spot-nozzle interaction was found to scale with the 
Chapter 6 - Entropy indirect noise Compared to Vortex driven INDIRECT NOISE IN LARGE SOLID ROCKET MOTORS: A NUMERICAL INVESTIGATION 149 OF THE INFLUENCE OF A NOZZLE CAVITY

static pressure difference $c^{2} \delta \rho$, the entropy-spot-core radius $R_{s}$, and the upstream Mach number $M$. The time scale of the duration of the response peak due to entropy-spot-nozzle interaction was found to be $R_{s} / U$. The entropyspot-nozzle interaction results imply that the time integral of the pressure pulse scales with the mass, $\delta m_{s}$, of the entropy spot. A physical explanation for this result has not been found.

The result that the presence of a nozzle cavity does not significantly influence entropy-spot-nozzle interaction induced upstream traveling pulse, in combination with the cold-gas scale experiments of Anthoine (Anthoine 2000) and the LP9 scale model experiments with combustion reported by Gallier et al. (Gallier et al. 2009), implies that the self-sustained pressure pulsations are primarily driven by vortex-nozzle indirect noise. This underscores the importance of the nozzle cavity volume, $V_{c}$, reported by (Anthoine et al. 2002; Gallier et al. 2009; Hirschberg et al. 2018). It is expected that in spite of the simplified model used in the simulations, viz. frictionless two-dimensional plane, the results described here illustrate the first-order contributions to sustained pressure pulsations in SRMs (at least in the LP9 scale model experiments). Further research is needed to confirm this expectation. However, the variation of parameters carried out in the present study indicates that the results are robust. 



\section{Conclusion}

\section{Summary of conclusions}

During the research described in this thesis, two reduced-order modeling strategies for limit-cycle pulsation amplitude prediction in large Solid Rocket Motors (SRMs) have been explored. These are a global lumped element approach and an energy balance approach.

The establishment of a limit cycle implies a nonlinear saturation process. In small SRM direct combustion noise is dominant and limit-cycle pulsations are on the order $p^{\prime} / p=\mathcal{O}\left(10^{-1}\right)$ (Fig. 1.8) (Flandro et al. 2007; Greatrix 2011). In this case dissipation in shockwaves is the main dissipation mechanism leading to limit-cycle saturation. In large SRMs, with pulsation amplitudes on the order $p^{\prime} / p=\mathcal{O}\left(10^{-3}\right)$, indirect noise due to vortex-nozzle interaction appears to be an important source of sound. The nonlinear saturation of this source is responsible for the attainment of a limit cycle. This is the result of the formation of discreet vortices with a circulation independent of the pulsation amplitude. When $p^{\prime} / p=\mathcal{O}\left(10^{-4}\right)$ this nonlinear saturation becomes weak and the amplitude of the limit cycle is very sensitive to small changes. Here even "high-fidelity" numerical simulations cannot predict limit-cycle amplitudes accurately. The models proposed here assume a strong saturation of vortex formation as encountered for $10^{-3} \leq p^{\prime} / p \leq 10^{-2}$, i.e. in the moderate amplitude regime. Such models overestimate the amplitude of pulsations when used in the low amplitude regime $p^{\prime} / p \leq 10^{-4}$.

In chapter 2, a system of harmonic oscillator equations for the pulsation amplitude due to vortex driven indirect sound has been derived. These harmonic oscillator equations are the first step in the direction of the aforementioned global lumped element model for the limit-cycle amplitude. The derivation uses a vortex sound analogy, for which the total enthalpy fluctuation $B^{\prime}$ is the natural aeroacoustic variable, similar that used by Howe (Howe 1998). The difference is that an extra source term, neglected by Howe, is used to correctly model the nozzle convection losses. The relevance of this term is confirmed by the analyzed results. 
High fidelity limit-cycle simulations and scale model experiments are onerous. Therefore, it was decided to do systematic dedicated investigations of the sound source due to vortex-nozzle interaction. From these scaling laws reduced-order models of the sound source were derived.

As a first step, an analytical incompressible plane two-dimensional model for vortex-nozzle interaction as a sound source was derived (chapter 3). This model is based on Howe's compact low-frequency Green's function method (Howe 2014). Howe proposes this method to model sound produced due to interaction of entropy spots, turbulence patches and vortices with contractions in pipe and channel flows. In the model derived in chapter 3, the nozzle is represented as a contraction. The shape of the contraction can be varied, by changing the contraction length $d / S_{1}$ where $S_{1}$ is the height of the upstream channel. A Green's function for a locally incompressible source region was derived.

Using this analytical incompressible model, a scaling law $B^{\prime} \propto \tilde{\Gamma}^{2}$ was found, where $\tilde{\Gamma} \equiv \Gamma /\left(U S_{1}\right)$ is dimensionless vortex circulation with $U$ and $S_{1}$ the upstream velocity and channel height, respectively. The interpretation of this scaling behavior can be summarized as follows: sound is produced on approach to the contraction in a locally incompressible acoustic velocity field, and occurs due to the vortex trajectory crossing the acoustic velocity field lines under the influence of an effective image vortex (representing the interaction with the wall). When compared to low Mach number compressible vortex-nozzle interaction Internal Aeroacoustics code (EIA) simulations reported by Hulshoff et al. (Hulshoff et al. 2001), satisfactory agreement of the results is found.

Hulshoff's simulations (Hulshoff et al. 2001) were carried out for cases without the presence of a nozzle cavity, mainly for subcritical nozzles. When selfsustained pressure pulsations occur in SRMs, the nozzle is critical (choked). Furthermore, commonly used SRMs such as the Ariane 5 P230 and the Titan 4 SRM have a so-called integrated nozzle (a nozzle with a surrounding cavity volume). The importance of this cavity volume, $V_{c}$, for vortex driven indirect sound was established by Anthoine (Anthoine 2000) using cold-gas-scale model experiments, and by Gallier et al. (Gallier et al. 2009) using a scale model with combustion of a non-metalized propellant.

Dedicated vortex-nozzle interaction simulations have been performed for nozzle inlet geometries with and without a nozzle cavity (chapter 4). In these simulation the nozzle is choked as it is in SRMs. The results of systematic parameter studies for vortex-nozzle interaction confirm the importance of the nozzle cavity volume. The simulations show that the acoustic response peak magnitude is linearly proportional to the nozzle cavity volume $V_{c}$. The simulations point to the importance of local compressibility in the source region. Namely, the compressibility of the fluid around the nozzle inlet and in the nozzle cavity 
volume when present. A scaling behavior $B^{\prime} \propto M \tilde{\Gamma}$, where $M \equiv U / c$ is the nozzle inlet Mach number, was discovered, indicating that, the local compressibility contribution to the acoustic response is linearly dependent on the vortex circulation. This is in contrast to the scaling behavior found for a locally incompressible source region, which scales with the square of the dimensionless vortex circulation. The linear scaling behavior dominates the acoustic response in the relevant range of vortex circulation $0.1 \leq|\tilde{\Gamma}|<1$ for SRM vortex-nozzle interaction. In the case of an integrated nozzle, it persists up to unrealistically high vortex circulations. Thus, Howe's compact low-frequency Green's function is insufficient to describe vortex-nozzle interaction as a source of sound.

In the following a qualitative explanation of vortex-nozzle interaction is provided. The general integral form of the aeroacoustic analogy due to the interaction of an inhomogeneity, such as a vortex or entropy spot, has the following form

$$
B^{\prime}=-\int_{-\Theta}^{\Theta} \int_{V}\left[\frac{\mathbf{f}}{\rho}\right] \cdot \nabla_{\mathbf{y}} G \mathrm{~d}^{3} y \mathrm{~d} \tau
$$

where $\mathbf{f}$ is a force density. When vortex-nozzle interaction is considered $\mathbf{f}=$ $\mathbf{f}_{v}=\rho[\boldsymbol{\omega} \times \mathbf{v}]$. Here $G$ is the Green's function, which Howe (Howe 1998) approximates as a compact low-frequency Green's function $G_{r}$. Motivated by the conclusion that local compressibility effects are dominant for indirect sound due to vortex-nozzle interaction, we proposed the following correction $G=G_{r}+G_{c}$, where $G_{c}$ is the Green's function contribution due to local compressibility effects. On approach to the nozzle a vortex interacts with the local acoustic velocity field $\nabla G$. For vortex circulations in the range of interest, sound production is mainly due to the fact that in first approximation, $\boldsymbol{\omega} \times \mathbf{v}$ has a large parallel component to $\nabla G_{c}$.

To model the local compressibility contribution due to fluid around the nozzle inlet, viz. outside the nozzle cavity, a residual volume $V_{0}$ is proposed (chapter 4). In the incompressible two-dimensional plane model, compressibility can be taken into account by placing a point source at the nozzle inlet. This induces an acoustic flux equivalent to the flux generated by a cavity of volume $V_{\text {eff }}=$ $V_{0}+V_{c}$. The scaling behavior of vortex-nozzle interaction sound in the range of interest is then: $B^{\prime} \propto \tilde{L}_{\text {eff }} M \tilde{\Gamma}$, where $\tilde{L}_{\text {eff }} \equiv V_{\text {eff }} /\left(4 S_{1}^{3}\right)=\left(V_{c}+V_{0}\right) /\left(4 S_{1}^{3}\right)$.

The scaling laws for the incompressible and compressible contributions to vortexnozzle interaction sound have been used to develop a lumped element model of the source with six fit parameters (chapter 4). For a roughly chosen set of fit parameters, satisfactory agreement with EIA (Euler Internal Aeroacoustics code appendix I) simulations is found. The lumped element source model is 
universally valid and could be implemented in an acoustical model of the SRM (e.g. the harmonic oscillator model described in chapter 2) to predict limitcycle oscillation amplitude, as has been done for the incompressible flow model (Hirschberg et al. 2018). Furthermore, the procedure followed here can be used to obtain a lumped element sound source model from more realistic numerical simulations e.g. axis-symmetrical flows or eventually three-dimensional turbulent flow.

In an attempt to reduce sound production, a gradual ramp inlet geometry has been considered (section 4.11). The change in geometry appears to reduce the radiation pulse amplitude by a factor two compared to amplitudes generated with a right angle inlet nozzle. However, the pulse duration increases by a factor two, implying that the time integral of the pulse, $\int B^{\prime} d t$, is approximately unchanged. For large SRMs, the duration of the pulse remains short compared to the longitudinal oscillation period of the combustion chamber $2 L / c$ (here $L$ is the length of the combustion chamber). Thus, there would be little benefit in using such a longer nozzle inlet for the reduction of the limit-cycle pulsation amplitude in SRMs. However, for configurations where the sound pulse length is comparable or larger than $2 L / c$, limit-cycle amplitude could be reduced. It is furthermore striking that compressibility effects are still quite important for the gradual ramp inlet configuration. Even in this case, scaling with the square of the dimensionless vortex circulation $\tilde{\Gamma}^{2}$ is only approached for unrealistically large vortex circulations $|\tilde{\Gamma}|>2$.

From the EIA simulations it appears that sound due to vortex-nozzle interaction is mainly generated during the approach phase.

In chapter 5, a semi-analytical model based on the energy-balance approach is proposed. The maximum vortex circulation is estimated using a moderate amplitude approximation (Nelson et al. 1983; Bruggeman 1987; Bruggeman et al. 1991). A single fit parameter is used to adjust the vortex circulation to a lower value, as circulation is expected to be diffused by turbulence when it makes its way from the point where it is shed to the source region. The model was compared to cold-gas axial injection scale experiments carried out by Anthoine (Anthoine 2000). Agreement within a factor three was found for the pulsation amplitudes in the moderate amplitude regime $10^{-3} \leq \frac{p^{\prime}}{p} \leq 10^{-2}$. It was also noted that when pulsation amplitudes are lower than the moderate amplitude regime, prediction using analytical methods becomes very difficult if not impossible. Even with high-fidelity numerical simulations the prediction of low amplitude limit-cycles is problematic (Gallier et al. 2009).

Entropy inhomogeneity (entropy-spot) driven indirect sound has been reported as a source of self-sustained pressure pulsations in turbines (Dowling and Mahmoudi 2015; Morgans and Duran 2016). To investigate the role of entropy 
spot-nozzle indirect sound on self-sustained pressure pulsations in SRMs, dedicated EIA simulations with and without the presence of a nozzle cavity have been carried out (chapter 6). The results of these simulations show that the nozzle cavity does not have a significant influence on entropy spot driven indirect sound.

Scale experiments with combustion of non-metalized solid propellant are reported by Gallier et al. (Gallier et al. 2009). These show that sustained pressure pulsations are very strongly influenced by the presence of a nozzle cavity. This information combined with Anthoine's finding for cold-gas-scale experiment (Anthoine 2000) and the dedicated EIA simulations reported in this work leads to the conclusion that in large SRMs, self-sustained pressure pulsations are dominated by vortex-nozzle interaction as a source of indirect sound.

\section{Suggestions for the application of results}

The scaling laws obtained for sound production by vortex-nozzle (chapter 4) and entropy-spot-nozzle (chapter 6) interaction can be used to analyze, interpolate or extrapolate results from detailed numerical simulations, scale experiments and full scale tests. As stated in the summary of conclusions these scaling laws are based on two-dimensional plane simulations performed with a frictionless model (EIA). To confirm their pertinence, in the future it will be necessary to investigate the effect of three-dimensional flow effects such as turbulence and self-induced vortex velocity. Also the interaction between successive vortices should be considered.

An important result is that sound production by vortex-nozzle interaction is mainly due to a local compressibility effect on approach of the vortex to the nozzle, rather than the final ingestion (interaction with the transonic throat region). This implies that modifications of the nozzle geometry are not crucial, except for the volume, $V_{c}$, of the nozzle cavity surrounding the nozzle inlet of an integrated nozzle.

Using the proposed quasi-steady linear models for losses of acoustic energy combined with the nonlinear sound source model in a global energy balance (as illustrated in chapter 5) one can predict pulsation amplitudes as a function of Mach number for specified acoustic modes. A detailed description of the acoustic field is not necessary. Fair results are obtained (order of magnitude estimates) assuming a simple acoustically closed-closed pipe model. Also the calculations involve a very limited numerical expense and can thus be repeated for the few expected longitudinal acoustic modes (first and second in P230 boosters (Fabignon et al. 2003)). An important uncertainty here is the 
estimate of the vortex circulation, $\Gamma$. A simplified model valid in the moderate amplitude regime has been used to find an upper bound $\Gamma_{\max }$. Thus, unless a more precise way of modeling $\Gamma$ is developed in the future, using this model as an engineering predesign tool will only give an order magnitude estimate or upper bound of expected pulsation amplitudes.

The first steps in the development of a global lumped element model to predict time evolution and acoustic mode selection in large SRMs due to vortex driven indirect sound are presented in chapter 2. This modeling approach corresponds to the one taken by Culick (Culick 2006) for direct combustion noise driven instabilities. It could actually be used to investigate the influence of vortexnozzle interaction and direct combustion noise in large SRMs using a single reduced order model.

When considering small SRMs for which direct combustion driven instabilities are assumed to be dominant, one has to account for amplitude limitation due to nonlinear wave steepening and eventually shockwave formation. In that case nonlinear vortex shedding and vortex-nozzle interaction has been ignored. We indicate in chapter 1 that nonlinear damping by vortex shedding scales with the third power of the pulsation amplitude (as does shock damping) (Howe 1984). It seems advisable to take this into account in such models. The proposed vortex-nozzle sound production lumped element model (chapter 4) could be used to asses the role of this sound production mechanism in these models for small SRM limit-cycle behavior. The lumped element source model could be integrated in one-dimensional gas dynamical models such as those proposed by Levine and Baum (Levine and Baum 1982; Baum and Levine 1986), Greatrix (Greatrix 2011) and Ferretti et al. (Ferretti et al. 2011; Ferretti 2011). These models include nonlinear wave steepening effects and eventual shockwaves. The fact that such models are quite successful indicates that the impact of details of the geometry on acoustical response of the combustion chamber is not crucial.

When considering hybrid rocket engines or delayed combustion of metal particles in SRMs, one can expect entropy non-uniformities significantly larger than the minimal characteristic inhomogeneity $\delta \rho / \rho \simeq(\gamma-1) M^{2} / 2$ which will always be present in vortices. The scale rule obtained in chapter 6 allows to estimate the effect of interaction with the nozzle of entropy inhomogeneities smaller than the combustion chamber radius. For larger inhomogeneities, with length scale of the order of the diameter of the chamber, one can use the theory of Marble and Candel (Marble and Candel 1977).

Globally, the work presented in this thesis should be viewed as the initial steps taken towards the development of engineering tools for the prediction of the influence of vortex shedding and vortex-nozzle interaction on the pressure pulsations in combustion chambers of SRMs. 


\section{Future research perspectives}

Below, a short list of possible future avenues of research is provided.

- Axis-symmetrical simulations of indirect noise due to both vortex-nozzle and entropy spot-nozzle interaction would be a first step. This would serve to verify the range over which two-dimensional plane model results remain pertinent.

- Interaction between successive vortices.

- Systematic numerical investigation of vortex shedding processes.

- Investigation of turbulence-nozzle interaction as a source of indirect noise.

- Study of viscous flow effects, such as possible boundary layer separation on the nozzle inlet wall.

- Implementation of lumped element source model in pulsation amplitude harmonic oscillator model (global lumped element model).

- Investigation of the influence of turbulence on vortex-nozzle and entropy spot-nozzle interaction. 



\section{Appendix A}

\section{Orthogonality condition}

We consider an acoustic mode $\psi_{m}$ with wave number $k_{m}$ satisfying the Helmholtz equation

$$
\nabla^{2} \psi_{m}=-k_{m}^{2} \psi_{m}
$$

with boundary conditions $\psi_{m}=0$ or $\nabla \psi_{m} \cdot \mathbf{n}=0$ on the control surface $S$ enclosing a volume $V$, with outer unit normal vector $\mathbf{n}$. In this appendix the orthogonality condition Eq. (2.21) is derived. Due to Eq. (2.20), it is obvious that

$$
\int_{V}\left(\psi_{m}\left(\nabla^{2} \psi_{n}+k_{n}^{2} \psi_{n}\right)-\psi_{n}\left(\nabla^{2} \psi_{m}+k_{m}^{2} \psi_{m}\right)\right) \mathrm{d}^{3} x=0
$$

consequently

$$
\int_{V}\left(\psi_{m} \nabla^{2} \psi_{n}-\psi_{n} \nabla^{2} \psi_{m}\right) \mathrm{d}^{3} x=\left(k_{n}^{2}-k_{m}^{2}\right) \int_{V} \psi_{n} \psi_{m} \mathrm{~d}^{3} x
$$

Integrating the lefthand side above by parts, yields

$$
\begin{aligned}
\int_{V}\left(\psi_{m} \nabla^{2} \psi_{n}-\psi_{n} \nabla^{2} \psi_{m}\right) \mathrm{d}^{3} x & =\int_{S}\left(\psi_{m} \nabla \psi_{n}-\psi_{n} \nabla \psi_{m}\right) \cdot \mathbf{n d} \mathrm{d}^{2} x \\
& -\int_{V}\left(\nabla \psi_{m} \cdot \nabla \psi_{n}-\nabla \psi_{n} \cdot \nabla \psi_{m}\right) \mathrm{d}^{3} x .
\end{aligned}
$$

The volume integral on the righthand side obviously vanishes and surface integral vanishes due to the imposed boundary conditions of the problem viz. 
$\psi_{n}=0$ or $\mathbf{n} \cdot \nabla \psi_{n}=0$ on the $S$. Thus form Eq. (A.3) it follows that

$$
\int_{V} \psi_{n} \psi_{m} \mathrm{~d}^{3} x=E_{n}^{2} \delta_{n m},
$$

where $E_{n}^{2}=\int_{V} \psi_{n}^{2} d^{3} x$ is normalization factor and $\delta_{n m}$ is a Kronecker delta $\left(\delta_{n m}=0\right.$ for $n \neq m$ and $\delta_{n m}=0$ for $\left.n=m\right)$. Eq. A.5 is identical to Eq. 2.21 in the main text. 


\section{Appendix B}

\section{Details derivation harmonic oscillator model}

In this appendix detailed algebraic steps for the derivation of the global lumped model omitted in the text are shown. The integral Eq. 2.25 for the total enthalpy fluctuation $B^{\prime}$ in terms of the Greens function $G$ is the starting point of the derivation. We expand both $G$ and $B^{\prime}$ in modes (Eg. (2.19) and (2.22), respectively) to obtain:

$$
\begin{aligned}
& B^{\prime}=\sum_{n} b_{n} \psi_{n}(\mathbf{x})= \\
& \sum_{n}\left(\frac{c_{0}}{E_{n}}\right)^{2} \int_{-\Theta}^{\Theta} \int_{S}\left[\mathcal{H}(t-\tau) \frac{\psi_{n}(\mathbf{x}) \psi_{n}(\mathbf{y}) \sin \left(\omega_{n}(t-\tau)\right)}{\omega_{n}} \nabla_{\mathbf{y}} B^{\prime}\right] \cdot \mathbf{n} \mathrm{d}^{2} y \mathrm{~d} \tau \\
&-\sum_{n}\left(\frac{c_{0}}{E_{n}}\right)^{2} \int_{-\Theta}^{\Theta} \int_{S}\left[B^{\prime} \mathcal{H}(t-\tau) \frac{\psi_{n}(\mathbf{x}) \nabla_{\mathbf{y}} \psi_{n}(\mathbf{y}) \sin \left(\omega_{n}(t-\tau)\right)}{\omega_{n}}\right] \cdot \mathbf{n} \mathrm{d}^{2} y \mathrm{~d} \tau \\
&-\sum_{n}\left(\frac{c_{0}}{E_{n}}\right)^{2} \int_{-\Theta}^{\Theta} \int_{V} \mathcal{H}(t-\tau) \frac{\psi_{n}(\mathbf{x}) \nabla_{\mathbf{y}} \psi_{n}(\mathbf{y}) \sin \left(\omega_{n}(t-\tau)\right)}{\omega_{n}} \cdot(\boldsymbol{\omega} \times \mathbf{v}) \mathrm{d}^{3} y \mathrm{~d} \tau \\
&-\sum_{n}\left(\frac{c_{0}}{E_{n}}\right)^{2} \int_{-\Theta}^{\Theta} \int_{S} \mathcal{H}(t-\tau) \frac{\psi_{n}(\mathbf{x}) \psi_{n}(\mathbf{y}) \sin \left(\omega_{n}(t-\tau)\right)}{\omega_{n}}\left(\frac{\mathbf{U} \cdot \mathbf{n}}{c_{0}^{2}} \frac{\partial B^{\prime}}{\partial \tau}\right) \mathrm{d}^{2} y \mathrm{~d} \tau
\end{aligned}
$$

here $\boldsymbol{\omega}$ is the vorticity, $\mathcal{H}(t-\tau)$ is a Heaviside function, $c_{0}$ is the observer state value of the speed of sound, $\mathbf{U}$ is the average main flow velocity, $\psi_{n}(\mathbf{x})$ is the acoustic mode and $\omega_{n}$ its frequency. The normalization coefficient $E_{n}^{2}$ is discussed in appendix A. Multiplying Eq. (B.1) by $\psi_{n}(\mathbf{x})$ ( $n^{\text {th }}$ acoustic mode) and integrating over the control volume $V$, using the orthogonality condition (Eq. (A.5) in the main text Eq. 2.21), one can find an equation for the amplitude $b_{n}=b_{n}(t)$ of the $n^{\text {th }}$ mode viz. 


$$
\begin{aligned}
b_{n} & =\left(\frac{c_{0}}{E_{n}}\right)^{2} \int_{-\Theta}^{\Theta} \int_{S}\left[\mathcal{H}(t-\tau) \frac{\psi_{n}(\mathbf{y}) \sin \left(\omega_{n}(t-\tau)\right)}{\omega_{n}} \nabla_{\mathbf{y}} B^{\prime}\right] \cdot \mathbf{n} \mathrm{d}^{2} y \mathrm{~d} \tau \\
& -\left(\frac{c_{0}}{E_{n}}\right)^{2} \int_{-\Theta}^{\Theta} \int_{V} \mathcal{H}(t-\tau) \frac{\nabla_{\mathbf{y}} \psi_{n}(\mathbf{y}) \sin \left(\omega_{n}(t-\tau)\right)}{\omega_{n}} \cdot(\boldsymbol{\omega} \times \mathbf{v}) \mathrm{d}^{3} y \mathrm{~d} \tau \\
& -\left(\frac{c_{0}}{E_{n}}\right)^{2} \int_{-\Theta}^{\Theta} \int_{S} \mathcal{H}(t-\tau) \frac{\psi_{n}(\mathbf{y}) \sin \left(\omega_{n}(t-\tau)\right)}{\omega_{n}}\left(\frac{\mathbf{U} \cdot \mathbf{n}}{c_{0}^{2}} \frac{\partial B^{\prime}}{\partial \tau}\right) \mathrm{d}^{2} y \mathrm{~d} \tau .
\end{aligned}
$$

To find the above result $\mathbf{n} \cdot \nabla_{\mathbf{y}} \psi_{n}(\mathbf{y})=0$ on $S$ was used.

Taking the the partial derivative with respect to time of Eq. (B.2), one finds

$$
\begin{array}{cl}
\frac{\partial B^{\prime}}{\partial t} & =\sum_{n}\left(\frac{c_{0}}{E_{n}}\right)^{2} \int_{-\Theta}^{\Theta} \int_{S}\left[\psi _ { n } ( \mathbf { x } ) \psi _ { n } ( \mathbf { y } ) \left(\delta(t-\tau) \frac{\sin \left(\omega_{n}(t-\tau)\right)}{\omega_{n}}\right.\right. \\
+ & \left.\left.\mathcal{H}(t-\tau) \cos \left(\omega_{n}(t-\tau)\right)\right)\right] \nabla_{\mathbf{y}} B^{\prime} \cdot \mathbf{n} \mathrm{d}^{2} y \mathrm{~d} \tau \\
-\sum_{n}\left(\frac{c_{0}}{E_{n}}\right)^{2} & \int_{-\Theta}^{\Theta} \int_{S}\left[B ^ { \prime } \psi _ { n } ( \mathbf { x } ) \left(\delta(t-\tau) \frac{\sin \left(\omega_{n}(t-\tau)\right)}{\omega_{n}}\right.\right. \\
+ & \left.\left.\mathcal{H}(t-\tau) \cos \left(\omega_{n}(t-\tau)\right)\right)\right] \nabla_{\mathbf{y}} \psi_{n}(\mathbf{y}) \cdot \mathbf{n} \mathrm{d}^{2} y \mathrm{~d} \tau \\
-\sum_{n}\left(\frac{c_{0}}{E_{n}}\right)^{2} & \int_{-\Theta}^{\Theta} \int_{V}(\boldsymbol{\omega} \times \mathbf{v}) \cdot \nabla_{\mathbf{y}}\left(\delta(t-\tau) \frac{\psi_{n}(\mathbf{x}) \psi_{n}(\mathbf{y}) \sin \left(\omega_{n}(t-\tau)\right)}{\omega_{n}}\right) \mathrm{d}^{3} y \mathrm{~d} \tau \\
-\sum_{n}\left(\frac{c_{0}}{E_{n}}\right)^{2} & \int_{-\Theta}^{\Theta} \int_{V}(\boldsymbol{\omega} \times \mathbf{v}) \cdot \nabla_{\mathbf{y}}\left(\mathcal{H}(t-\tau) \psi_{n}(\mathbf{x}) \psi_{n}(\mathbf{y}) \cos \left(\omega_{n}(t-\tau)\right)\right) \mathrm{d}^{3} y \mathrm{~d} \tau \\
-\sum_{n}\left(\frac{c_{0}}{E_{n}}\right)^{2} & \int_{-\Theta}^{\Theta} \int_{S} \delta(t-\tau) \frac{\psi_{n}(\mathbf{x}) \psi_{n}(\mathbf{y}) \sin \left(\omega_{n}(t-\tau)\right)}{\omega_{n}}\left(\frac{\mathbf{U} \cdot \mathbf{n}}{c_{0}^{2}} \frac{\partial B^{\prime}}{\partial \tau}\right) \mathrm{d}^{2} y \mathrm{~d} \tau \\
-\sum_{n}\left(\frac{c_{0}}{E_{n}}\right)^{2} & \int_{-\Theta}^{\Theta} \int_{S} \mathcal{H}(t-\tau) \psi_{n}(\mathbf{x}) \psi_{n}(\mathbf{y}) \cos \left(\omega_{n}(t-\tau)\right)\left(\frac{\mathbf{U} \cdot \mathbf{n}}{c_{0}^{2}} \frac{\partial B^{\prime}}{\partial \tau}\right) \mathrm{d}^{2} y \mathrm{~d} \tau
\end{array}
$$

Using the fact that under the integral $\delta(t-\tau) \sin \left(\omega_{n}(t-\tau)\right)$ terms vanish, the above equation reduces to 


$$
\begin{aligned}
\frac{\partial B^{\prime}}{\partial t} & =\sum_{n}\left(\frac{c_{0}}{E_{n}}\right)^{2} \int_{-\Theta}^{\Theta} \int_{S} \mathcal{H}(t-\tau) \psi_{n}(\mathbf{x}) \cos \left(\omega_{n}(t-\tau)\right)\left[\psi_{n}(\mathbf{y}) \nabla_{\mathbf{y}} B^{\prime}\right. \\
& \left.-B^{\prime} \nabla_{\mathbf{y}} \psi_{n}(\mathbf{y})\right] \cdot \mathbf{n} \mathrm{d}^{2} y \mathrm{~d} \tau \\
& -\sum_{n}\left(\frac{c_{0}}{E_{n}}\right)^{2} \int_{-\Theta}^{\Theta} \int_{V} \mathcal{H}(t-\tau) \psi_{n}(\mathbf{x}) \cos \left(\omega_{n}(t-\tau)\right)(\boldsymbol{\omega} \times \mathbf{v}) \cdot \nabla_{\mathbf{y}} \psi_{n}(\mathbf{y}) \mathrm{d}^{3} y \mathrm{~d} \tau \\
& -\sum_{n}\left(\frac{c_{0}}{E_{n}}\right)^{2} \int_{-\Theta}^{\Theta} \int_{S} \mathcal{H}(t-\tau) \psi_{n}(\mathbf{x}) \psi_{n}(\mathbf{y}) \cos \left(\omega_{n}(t-\tau)\right) \frac{\mathbf{U} \cdot \mathbf{n}}{c_{0}^{2}} \frac{\partial B^{\prime}}{\partial \tau} \mathrm{d}^{2} y \mathrm{~d} \tau .
\end{aligned}
$$

Taking the second partial derivative of the stagnation enthalpy fluctuation $B^{\prime}$ with respect to time, yields

$$
\begin{aligned}
\frac{\partial^{2} B^{\prime}}{\partial t^{2}} & =\sum_{n}\left(\frac{c_{n}}{E_{0}}\right)^{2} \psi_{n}(\mathbf{x}) \frac{\mathrm{d}^{2} b_{n}}{\mathrm{~d} t^{2}}= \\
- & \sum_{n}\left(\frac{c_{0}}{E_{n}}\right)^{2} \psi_{n}(\mathbf{x}) \omega_{n}^{2} \int_{-\Theta}^{\Theta} \int_{S} \mathcal{H}(t-\tau) \frac{\sin \left(\omega_{n}(t-\tau)\right)}{\omega_{n}}\left(\psi_{n}(\mathbf{y}) \nabla_{\mathbf{y}} B^{\prime}\right. \\
& \left.-B^{\prime} \nabla_{\mathbf{y}} \psi_{n}(\mathbf{y})\right) \cdot \mathbf{n} \mathrm{d}^{2} y \mathrm{~d} \tau \\
+ & \sum_{n}\left(\frac{c_{0}}{E_{n}}\right)^{2} \psi_{n}(\mathbf{x}) \omega_{n}^{2} \int_{-\Theta}^{\Theta} \mathcal{H}(t-\tau) \frac{\sin \left(\omega_{n}(t-\tau)\right)}{\omega_{n}}(\boldsymbol{\omega} \times \mathbf{v}) \cdot \nabla_{\mathbf{y}} \psi_{n}(\mathbf{y}) \mathrm{d}^{3} y \mathrm{~d} \tau \\
+ & \sum_{n}\left(\frac{c_{0}}{E_{n}}\right)^{2} \psi_{n}(\mathbf{x}) \omega_{n}^{2} \int_{-\Theta}^{\Theta} \int_{S} \mathcal{H}(t-\tau) \frac{\sin \left(\omega_{n}(t-\tau)\right)}{\omega_{n}} \psi_{n}(\mathbf{y}) \frac{\mathbf{U} \cdot \mathbf{n}}{c_{0}^{2}} \frac{\partial B^{\prime}}{\partial \tau} \mathrm{d}^{2} y \mathrm{~d} \tau \\
+ & \sum_{n}\left(\frac{c_{0}}{E_{n}}\right)^{2} \psi_{n}(\mathbf{x}) \int_{S}\left(\psi_{n}(\mathbf{y}) \nabla_{\mathbf{y}} B^{\prime}-B^{\prime} \nabla_{\mathbf{y}} \psi_{n}(\mathbf{y})\right) \cdot \mathbf{n} \mathrm{d}^{2} y \\
- & \sum_{n}\left(\frac{c_{0}}{E_{n}}\right)^{2} \psi_{n}(\mathbf{x}) \int_{V}(\boldsymbol{\omega} \times \mathbf{v}) \cdot \nabla_{\mathbf{y}} \psi_{n}(\mathbf{y}) \mathrm{d}^{3} y \\
& -\sum_{n}\left(\frac{c_{0}}{E_{n}}\right)^{2} \psi_{n}(\mathbf{x}) \int_{S} \psi_{n}(\mathbf{y}) \frac{\mathbf{U} \cdot \mathbf{n}}{c_{0}^{2}} \frac{\partial B^{\prime}}{\partial \tau} \mathrm{d}^{2} y
\end{aligned}
$$

Recognizing that the first three sums equal $\sum_{n}-\omega_{n}^{2} b_{n} \psi_{n}(\mathbf{x})$ (see Eq. (B.2)), one rewrites to find 


$$
\begin{aligned}
& \sum_{n}\left(\frac{c_{0}}{E_{n}}\right)^{2} \psi_{n}(\mathbf{x})\left(\frac{\mathrm{d}^{2} b_{n}}{\mathrm{~d} t^{2}}+\omega_{n}^{2} b_{n}\right)= \\
+ & \sum_{n}\left(\frac{c_{0}}{E_{n}}\right)^{2} \psi_{n}(\mathbf{x}) \int_{S}\left(\psi_{n}(\mathbf{y}) \nabla_{\mathbf{y}} B^{\prime}-B^{\prime} \nabla_{\mathbf{y}} \psi_{n}(\mathbf{y})\right) \cdot \mathbf{n} \mathrm{d}^{2} y \\
- & \sum_{n}\left(\frac{c_{0}}{E_{n}}\right)^{2} \psi_{n}(\mathbf{x}) \int_{V}(\boldsymbol{\omega} \times \mathbf{v}) \cdot \nabla_{\mathbf{y}} \psi_{n}(\mathbf{y}) \mathrm{d}^{3} y \\
- & \sum_{n}\left(\frac{c_{0}}{E_{n}}\right)^{2} \psi_{n}(\mathbf{x}) \int_{S} \psi_{n}(\mathbf{y}) \frac{\mathbf{U} \cdot \mathbf{n}}{c_{0}^{2}} \frac{\partial B^{\prime}}{\partial \tau} \mathrm{d}^{2} y
\end{aligned}
$$

This result is identical to Eq. (2.33) in the main text. 


\section{Appendix C}

\section{Ratio of volume integral to surface integral}

Estimation of ratio of volume integral and surface integral in Eq. (2.30):

$$
I_{4}=\int_{-\Theta}^{\Theta} \int_{V} \nabla_{\mathbf{y}} G \cdot \frac{\mathbf{U}}{c_{0}^{2}} \frac{\partial B^{\prime}}{\partial \tau} d^{3} y d \tau-\int_{-\Theta}^{\Theta} \int_{S} \frac{G(\mathbf{U} \cdot \mathbf{n})}{c_{0}^{2}} \frac{\partial B^{\prime}}{\partial \tau} \mathrm{d}^{2} y \mathrm{~d} \tau
$$

here $B^{\prime}$ is the total enthalpy fluctuation, $G$ Greens function, $c_{0}$ is the observer state value of the speed of sound, $\mathbf{U}$ the average main flow velocity in the observer state. The ratio $\Xi$ is defined

$$
\Xi \equiv \frac{\int_{-\Theta}^{\Theta} \int_{V} \nabla_{\mathbf{y}} G \cdot \frac{\mathbf{U}}{c_{0}^{2}} \frac{\partial B^{\prime}}{\partial \tau} \mathrm{d}^{3} y \mathrm{~d} \tau}{\int_{-\Theta}^{\Theta} \int_{A} \frac{G(\mathbf{U} \cdot \mathbf{n})}{c_{0}^{2}} \frac{\partial B^{\prime}}{\partial \tau} \mathrm{d}^{2} y \mathrm{~d} \tau}
$$

The surface integral is limited to the nozzle inlet surface A. Hence the volume integral is compared to the convection losses at the nozzle inlet. For the Green's function $G$ the expansion in orthogonal modes Eq. (2.19):

$$
G=\sum_{n}\left(\frac{c_{0}}{E_{n}}\right)^{2} \frac{\mathcal{H}(t-\tau) \psi_{n}(\mathbf{x}) \psi_{n}(\mathbf{y}) \sin \left(\omega_{n}(t-\tau)\right)}{\omega_{n}}
$$

is substituted. Here $\psi_{n}(\mathbf{x})$ and $b_{n}$ are the $n^{\text {th }}$ acoustic mode and the amplitude of the $n^{t h}$ mode, respectively. The solution $B^{\prime}$ is expanded in orthogonal modes as well

$$
B^{\prime}=\sum_{n} b_{n}(t) \psi_{n}(\mathbf{x})
$$

furthermore the flow is assumed quasi-parallel $\mathbf{U} \simeq(U, 0,0)$ with $|\mathbf{U}|=U$. At low frequencies the Green's function and the acoustic fields are both uniform 
over the cross-section of the combustion chamber viz. plane acoustic waves prevail. For simplicity one assumes $U$ is self-similar i.e. $U=F(x) V(r)$ where $x$ and $r$ the axial and radial coordinates respectively. Radial injection is a linear function of the distance $x$ from the headend: $F(x)=M c_{0} x / L$ with $L$ the axial length of the chamber. While $V(r)$ is a cosine flow profile (Anthoine 2000; Griffond and Casalis 2000) (Taylor-Culick flow).

A single dominant mode $n$ is assumed (as observed empirically). Both integrands in $\Xi$ have the same time dependence. Thus the time integrals cancel out, the integrals over the radial coordinate $r$ cancel too because of self-similarity of the velocity profile. One is left with

$$
\Xi=\frac{\int_{0}^{L} \frac{\partial \psi_{n}(y)}{\partial y} \psi_{n}(y) U(y) \mathrm{d} y}{\psi_{n}^{2}(L) U(L)} .
$$

For a combustion chamber of length $L$ with uniform cross-section one has: $\psi_{n}=\cos (n \pi y / L)$ while $\frac{\partial \psi_{n}(y)}{\partial y} \sim \sin (n \pi / L)$. Thus $\Xi=0$ when $U$ is independent of the position $y$ (source coordinate along the axis of the combustion chamber), this is the case for axial injection.

In the case of radial injection one has $U(y)=M c_{0} y / L$, with $M$ the Mach number of the flow at the inlet of the chocked nozzle at $y=L$. Assuming a single mode $n$ to be dominant, one finds

$$
\begin{aligned}
\Xi & =-n \frac{\pi}{L} \int_{0}^{L} \frac{y}{L} \sin \left(n \frac{\pi y}{L}\right) \cos \left(n \frac{\pi y}{L}\right) \mathrm{d} y \\
& =-\frac{1}{2(2 n)^{2} \pi} \int_{0}^{2 n \pi} \xi \sin \xi d \xi=\frac{1}{4 n}
\end{aligned}
$$

where $\xi=2 n \pi y / L$ and $\sin \xi=2 \sin (\xi / 2) \cos (\xi / 2)$ is used.

The integral

$$
\int_{-\Theta}^{\Theta} \int_{A} \frac{G(\mathbf{U} \cdot \mathbf{n})}{c_{0}^{2}} \frac{\partial B^{\prime}}{\partial \tau} \mathrm{d}^{2} y \mathrm{~d} \tau
$$

corresponds to the convective radiation losses at the nozzle. This is the main loss term. Hence, for $n=1$, neglecting the volume integral corresponds to neglecting a term of the order of $25 \%$ of the convective radiation losses at the nozzle. This is a crude but reasonable approximation in view of uncertainties in the sound source model. However, with increasing mode number $n$, the approximation becomes much better. For $n=2$ e.g. one neglects a term of the order of $10 \%$. 


\section{Appendix D}

\section{Schwartz-Christoffel transformation}

In this appendix we derive the explicit mapping function for a backward facing step. To do this we follow the derivation described by Milne-Thomson's (MilneThomson 1968). Using the result obtained for the backward facing step we will take Henrici's (Henrici 1974) approach and derive the explicit mapping function for a contraction with a rounded edge.

We seek to find a mapping function of a backward facing step in the $z$-plane. Point $A$ at $z=\infty+i S_{1}$ is mapped at $\zeta \rightarrow-\infty$. We choose to map point $B$ at $z \rightarrow-\infty$ to the origin $\zeta=0$ in the $\zeta$-plane, see figure D.1. Points $C$ and $D$ are mapped to $\zeta=1$ and $\zeta=a$ respectively. To do that we use the following the Schwartz-Christoffel transformation:

$$
\begin{aligned}
\frac{\mathrm{d} z}{\mathrm{~d} \zeta} & =K \frac{1}{\zeta}\left[\frac{1}{(\zeta-1)^{-1 / 2}}\right] \frac{1}{(\zeta-a)^{1 / 2}} \\
& =K \frac{1}{\zeta} \sqrt{\frac{\zeta-1}{\zeta-a}}
\end{aligned}
$$

Thus at $\zeta=0$, we now have a sink for which, we have the following complex potential

$$
F=-\frac{U S_{1}}{\pi} \ln (\zeta)
$$

The complex conjugate of the velocity is 
168

$$
\begin{aligned}
V^{*} & =\frac{\mathrm{d} F}{\mathrm{~d} z} \\
& =\frac{1}{\frac{\mathrm{d} z}{\mathrm{~d} \zeta}} \frac{\mathrm{d} F}{\mathrm{~d} \zeta} \\
& =\frac{-U S_{1}}{\pi K} \sqrt{\frac{\zeta-a}{\zeta-1}} .
\end{aligned}
$$

At point E (see figure D.1) corresponding to $z \rightarrow \infty$ and $\zeta \rightarrow \infty$, we have

$$
V^{*}=-U \text {. }
$$

Which for $\zeta \rightarrow \infty$ by virtue of equation (D.6) is equal to

$$
V^{*}=-\frac{U S_{1}}{\pi K}
$$

from which it follows that

$$
K=\frac{S_{1}}{\pi} .
$$

The complex conjugate of the velocity thus becomes

$$
V^{*}=-U \sqrt{\frac{\zeta-a}{\zeta-1}}
$$

At point $\mathrm{B}$ corresponding to $z \rightarrow-\infty$ and $\zeta=0$, we have

$$
V^{*}=-U \frac{S_{1}}{S_{2}}=-U \sqrt{a}
$$

i.e.

$$
a=\left(\frac{S_{1}}{S_{2}}\right)^{2} .
$$

The following variables $\tau$ and $b$ are introduced

$$
\begin{aligned}
\tau^{2} & =\frac{\zeta-a}{\zeta-1} \\
b^{2} & =a
\end{aligned}
$$


such that

$$
\zeta=\frac{b^{2}-\tau^{2}}{1-\tau^{2}}
$$

Taking the natural logarithm on both sides and differentiating, yields

$$
\frac{\mathrm{d} \zeta}{\zeta}=2 \tau\left(\frac{1}{1-\tau^{2}}-\frac{1}{a-\tau^{2}}\right) \mathrm{d} \tau
$$

Using the chain rule, we have

$$
\frac{\mathrm{d} z}{\mathrm{~d} \zeta}=\frac{\mathrm{d} z}{\mathrm{~d} \tau} \frac{\mathrm{d} \tau}{\mathrm{d} \zeta}
$$

i.e.

$$
\begin{aligned}
\frac{\mathrm{d} z}{\mathrm{~d} \tau} & =\frac{\mathrm{d} z}{\mathrm{~d} \zeta} \frac{\mathrm{d} \zeta}{\mathrm{d} \tau} \\
& =2 \frac{S_{1}}{\pi}\left(\frac{1}{1-\tau^{2}}-\frac{1}{b^{2}-\tau^{2}}\right) \\
& =\frac{S_{1}}{\pi}\left(\frac{1}{1-\tau}+\frac{1}{1+\tau}-\frac{1}{b}\left(\frac{1}{b+\tau}-\frac{1}{b-\tau}\right)\right) .
\end{aligned}
$$

We integrate the result above to find

$$
z=\frac{S_{1}}{\pi}\left(\ln \left(\frac{1+\tau}{1-\tau}\right)-\frac{1}{b} \ln \left(\frac{b+\tau}{b-\tau}\right)\right)+L
$$

where $L$ is an integration constant. We determine $L$ by setting $\zeta=a=b^{2}$ or equivalently $\tau=0$ (equation D.13), we have

$$
z=0
$$

i.e.

$$
L=0 .
$$

The explicit mapping for a backward facing step is thus

$$
z=\frac{S_{1}}{\pi}\left(\ln \left(\frac{1+\tau}{1-\tau}\right)-\frac{1}{b} \ln \left(\frac{b+\tau}{b-\tau}\right)\right) .
$$


An explicit mapping for a rounded contraction edge is needed. To find one Henrici's approach (Henrici 1974) is used. This consists of replacing the factor $\sqrt{\zeta-1}$ (between square brackets in equation (D.1)) in equation for the differential form of the transformation for a sharp contraction, by $\alpha \sqrt{\zeta-1}+\beta \sqrt{\zeta-a}$, where $\alpha$ and $\beta$ are constants. We have

$$
\begin{aligned}
\frac{\mathrm{d} z}{\mathrm{~d} \zeta} & =\frac{1}{\zeta}[\alpha \sqrt{\zeta-1}+\beta \sqrt{\zeta-a}] \frac{1}{(\zeta-a)^{1 / 2}} \\
& =\left(\frac{\alpha}{\zeta} \sqrt{\frac{\zeta-1}{\zeta-a}}+\frac{\beta}{\zeta}\right) .
\end{aligned}
$$

Which after integration, yields

$$
z=\alpha\left[\ln \left(\frac{1+\tau}{1-\tau}\right)-\frac{1}{b} \ln \left(\frac{b+\tau}{b-\tau}\right)\right]+\beta \ln (\zeta)+L
$$

Where $L$ is an integration constant, which we determine by setting $\zeta=a=b^{2}$ or equivalently $\tau=0$, we find

$$
L=-\beta \ln \left(b^{2}\right)
$$

Hence, an explicit mapping for a smooth contraction is

$$
z=\alpha\left[\ln \left(\frac{1+\tau}{1-\tau}\right)-\frac{1}{b} \ln \left(\frac{b+\tau}{b-\tau}\right)\right]+\beta \ln \left(\frac{\zeta}{b^{2}}\right)
$$

The complex flow potential in absence of vortices is for this case (same as for backward step)

$$
F=-\frac{U S_{1}}{\pi} \ln (\zeta)
$$

I.e. the complex conjugate of the velocity is

$$
\begin{aligned}
V^{*} & =\frac{\mathrm{d} F}{\mathrm{~d} z} \\
& =-\frac{U S_{1}}{\pi \zeta} \frac{\mathrm{d} \zeta}{\mathrm{d} z} \\
& =-\frac{U S_{1}}{\pi}\left(\alpha \sqrt{\frac{\zeta-1}{\zeta-a}}+\beta\right)^{-1}
\end{aligned}
$$

To determine the constants $\alpha$ and $\beta$, consider $\zeta \rightarrow \infty(z \rightarrow \infty)$ and $\zeta=0$ (point $z \rightarrow-\infty$ ), for which one has respectively 


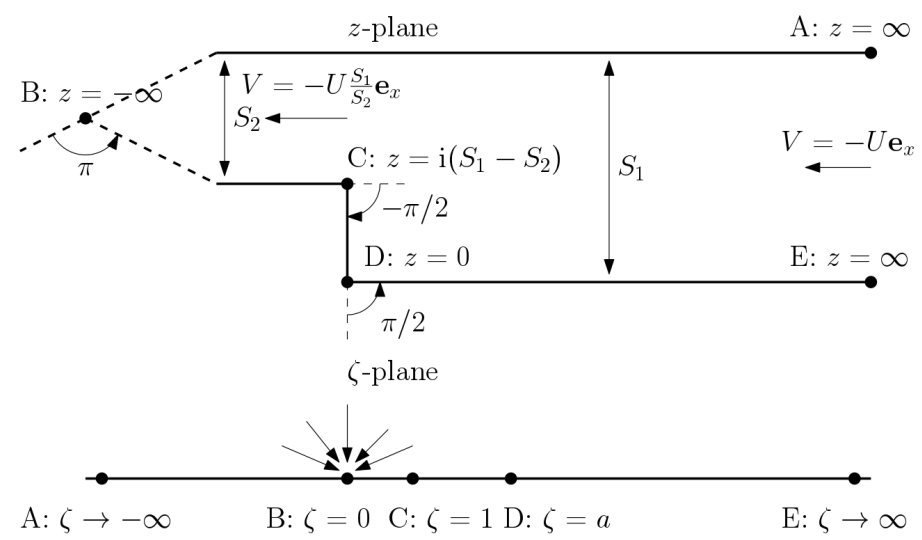

Figure D.1: Mapping of an abrupt contraction of a channel from cross section $S_{1}$ to $S_{2}$ in the z-plane to the real axis of the $\zeta$-plane.

$$
\begin{aligned}
V^{*}=-U & =-\frac{U S_{1}}{\pi}(\alpha+\beta)^{-1} \\
V^{*}=-U \frac{S_{1}}{S_{2}} & =-\frac{U S_{1}}{\pi}\left(\frac{\alpha}{b}+\beta\right)^{-1} .
\end{aligned}
$$

This is rewritten to find

$$
\begin{aligned}
\alpha+\beta & =\frac{U S_{1}}{\pi} \\
\frac{\alpha}{b}+\beta & =\frac{U S_{2}}{\pi} .
\end{aligned}
$$

Solving for $\alpha$ and $\beta$, yields

$$
\begin{gathered}
\alpha=\frac{S_{1}-S_{2}}{\pi}\left(\frac{b}{b-1}\right) \\
\beta=\frac{b S_{2}-S_{1}}{\pi(b-1)} .
\end{gathered}
$$





\section{Appendix E}

\section{Vortex path in two-dimensional incompressible flow theory}

In all cases considered one assumes a sink flow, with $Q=-U S_{1}$ at $\zeta=0$. A point vortex of circulation $\Gamma$ is placed at position $z_{\Gamma}$ corresponding to $\zeta_{\Gamma}$ as well as its mirror image in the real axis of the $\zeta$-plane at $\zeta_{\Gamma}^{*}$. The potential $\mathcal{F}(\zeta)$ of the flow in the $\zeta$-plane becomes

$$
\begin{aligned}
F(z) & =\mathcal{F}(\zeta) \\
& =-\frac{U S_{1}}{\pi} \ln (\zeta)-\mathrm{i} \frac{\Gamma}{2 \pi} \ln \left(\frac{\zeta-\zeta_{\Gamma}}{\zeta-\zeta_{\Gamma}^{*}}\right)
\end{aligned}
$$

The vortex is convected with the local flow velocity $V_{\Gamma}$ at the position $z_{\Gamma}$. To calculate $V_{\Gamma}$ using $\mathcal{F}(\zeta)$ take the own potential of the vortex into account, which becomes undetermined as $z$ approaches $z_{\Gamma}$. Viz. one should use the potential

to find

$$
\begin{aligned}
\mathcal{G}(\zeta) & \equiv G(z) \\
& =F(z)+\mathrm{i} \frac{\Gamma}{2 \pi} \ln \left(z-z_{\Gamma}\right)
\end{aligned}
$$

$$
\begin{aligned}
V_{\Gamma}^{*} & =\lim _{z \rightarrow z_{\Gamma}} \frac{\mathrm{d} G}{\mathrm{~d} z} \\
& =\lim _{z \rightarrow z_{\Gamma}} \frac{\mathrm{d} \mathcal{G}}{\mathrm{d} \zeta} \frac{\mathrm{d} \zeta}{\mathrm{d} z} \\
& =\lim _{z \rightarrow z_{\Gamma}}\left\{\frac{\mathrm{d} \zeta}{\mathrm{d} z}\left[-\frac{U S_{1}}{\pi \zeta}-\mathrm{i} \frac{\Gamma}{2 \pi} \frac{\mathrm{d}}{\mathrm{d} z} \ln \left(\frac{\zeta-\zeta_{\Gamma}}{z-z_{\Gamma}}\right)+\mathrm{i} \frac{\Gamma}{2 \pi\left(\zeta-\zeta_{\Gamma}^{*}\right)}\right]\right\} .
\end{aligned}
$$

The conformal mapping is defined by $\zeta=f(z)$. Expanding $f(z)$ in a Taylor series around $z_{\Gamma}$, yields 


$$
\begin{aligned}
\zeta-\zeta_{\Gamma} & =f(z)-f\left(z_{\Gamma}\right) \\
& =\left(z-z_{\Gamma}\right)\left(\frac{\mathrm{d} f}{\mathrm{~d} z}\right)_{z_{\Gamma}}+\frac{1}{2}\left(z-z_{\Gamma}\right)^{2}\left(\frac{\mathrm{d}^{2} f}{\mathrm{~d} z^{2}}\right)_{z_{\Gamma}}+\ldots
\end{aligned}
$$

Which can be rewritten to find

$$
\frac{\zeta-\zeta_{\Gamma}}{z-z_{\Gamma}}=\left(\frac{\mathrm{d} f}{\mathrm{~d} z}\right)_{z_{\Gamma}}+\frac{1}{2}\left(z-z_{\Gamma}\right)\left(\frac{\mathrm{d}^{2} f}{\mathrm{~d} z^{2}}\right)_{z_{\Gamma}}+\ldots
$$

In the limit $z \rightarrow z_{\Gamma}$, one finds

$$
\begin{aligned}
\lim _{z-z_{\Gamma}} \frac{\mathrm{d}}{\mathrm{d} z} \ln \left(\frac{\zeta-\zeta_{\Gamma}}{z-z_{\Gamma}}\right) & =\lim _{z-z_{\Gamma}} \frac{\frac{\mathrm{d}}{\mathrm{d} z}\left(\frac{\zeta-\zeta_{\Gamma}}{z-z_{\Gamma}}\right)}{\left(\frac{\zeta-\zeta_{\Gamma}}{z-z_{\Gamma}}\right)} \\
& =\frac{\left[\frac{\mathrm{d}}{\mathrm{d} z}\left(\frac{\mathrm{d} \zeta}{\mathrm{d} z}\right)\right]_{z_{\Gamma}}}{\left(\frac{\mathrm{d} \zeta}{\mathrm{d} z}\right)_{z_{\Gamma}}}=\frac{\left(\frac{\mathrm{d}^{2} \zeta}{\mathrm{d} z^{2}}\right)_{z_{\Gamma}}}{\left(\frac{\mathrm{d} \zeta}{\mathrm{d} z}\right)_{z_{\Gamma}}}
\end{aligned}
$$

This yields an expression for the complex conjugate of the vortex velocity $V_{\Gamma}^{*}$ with the so-called Routh correction (Milne-Thomson 1968; Saffman 1992; Clements 1973) (correction for the velocity at the position of the vortex)

$$
\begin{aligned}
V_{\Gamma}^{*} & =\left(\frac{\mathrm{d} z_{\Gamma}}{\mathrm{d} t}\right)^{*} \\
& =\left(\frac{\mathrm{d} \zeta}{\mathrm{d} z}\right)_{z_{\Gamma}}\left(-\frac{U S_{1}}{\pi \zeta_{\Gamma}}+\mathrm{i} \frac{\Gamma}{2 \pi\left(\zeta_{\Gamma}-\zeta_{\Gamma}^{*}\right)}\right)-\mathrm{i} \frac{\Gamma}{4 \pi} \frac{\left(\frac{\mathrm{d}^{2} \zeta}{\mathrm{d} z^{2}}\right)_{z_{\Gamma}}}{\left(\frac{\mathrm{d} \zeta}{\mathrm{d} z}\right)_{z_{\Gamma}}} .
\end{aligned}
$$

To evaluate the Routh correction (last term on the lefthand side in the equation above) in the $\zeta$-plane, one uses the following identity

$$
\begin{aligned}
\frac{\left(\frac{\mathrm{d}^{2} \zeta}{\mathrm{d} z^{2}}\right)}{\left(\frac{\mathrm{d} \zeta}{\mathrm{d} z}\right)} & =\left(\frac{\mathrm{d}}{\mathrm{d} \zeta} \ln \left(\frac{\mathrm{d} \zeta}{\mathrm{d} z}\right)\right) \frac{\mathrm{d} \zeta}{\mathrm{d} z} \\
& =-\frac{\left(\frac{\mathrm{d}}{\mathrm{d} \zeta} \ln \left(\frac{\mathrm{d} z}{\mathrm{~d} \zeta}\right)\right)}{\frac{\mathrm{d} z}{\mathrm{~d} \zeta}} \\
& =-\frac{\frac{\mathrm{d}^{2} z}{\mathrm{~d} \zeta^{2}}}{\left(\frac{\mathrm{d} z}{\mathrm{~d} \zeta}\right)^{2}}
\end{aligned}
$$

Using the conformal mapping of Henrici (Henrici 1974)

$$
\frac{\mathrm{d} z}{\mathrm{~d} \zeta}=\zeta^{-1}\left[\alpha(\zeta-1)^{1 / 2}+\beta(\zeta-a)^{1 / 2}\right](\zeta-a)^{-1 / 2}
$$


with

$$
\frac{\mathrm{d}^{2} z}{\mathrm{~d} \zeta^{2}}=-\left(\frac{1}{\zeta}+\frac{1}{2(\zeta-a)}\right) \frac{\mathrm{d} z}{\mathrm{~d} \zeta}+\frac{1}{2 \zeta}\left(\frac{\alpha}{\sqrt{\zeta-a} \sqrt{\zeta-1}}+\frac{\beta}{\zeta-a}\right) .
$$

From which on can find an explicit expression for the Routh correction as a function of $\zeta_{\Gamma}$.

The vortex is convected with the following velocity

$$
V_{\Gamma}=\frac{\mathrm{d} z_{\Gamma}}{\mathrm{d} t}
$$

Which is the conjugate of equation (E.13). To obtain the path in the $\zeta$-plane we write

$$
\begin{aligned}
\frac{\mathrm{d} \zeta_{\Gamma}}{\mathrm{d} t} & =\frac{\frac{\mathrm{d} z_{\Gamma}}{\mathrm{d} t}}{\frac{\mathrm{d} z}{\mathrm{~d} \zeta}} \\
& =\frac{\left(\frac{\mathrm{d} G}{\mathrm{~d} z}\right)_{\Gamma}^{*}}{\frac{\mathrm{d} z}{\mathrm{~d} \zeta}} \\
& =\frac{\left(\frac{\mathrm{d} G}{\mathrm{~d} \zeta}\right)_{\Gamma}^{*}}{\left|\frac{\mathrm{d} z}{\mathrm{~d} \zeta}\right|^{2}} \\
\frac{\mathrm{d} \zeta_{\Gamma}}{\mathrm{d} t} & =\frac{1}{\left|\frac{\mathrm{d} z}{\mathrm{~d} \zeta}\right|^{2}}\left[\left(-\frac{U S_{1}}{\pi \zeta_{\Gamma}}+\mathrm{i} \frac{\Gamma}{2 \pi\left(\zeta_{\Gamma}-\zeta_{\Gamma}^{*}\right)}\right)+\mathrm{i} \frac{\Gamma}{4 \pi} \frac{\left(\frac{\mathrm{d}^{2} z}{\mathrm{~d} \zeta^{2}}\right)_{\zeta_{\Gamma}}}{\left(\frac{\mathrm{d} z}{\mathrm{~d} \zeta}\right)_{\zeta_{\Gamma}}}\right]^{*} .
\end{aligned}
$$

Clements (Clements 1973) compares a simple first order Newton integration with a second order numerical integration. Howe (Howe 2014) proposes a 4th order Runge-Kutta integration to determine the vortex path by numerical integration in the $\zeta$-plane.

$$
\frac{\mathrm{d} \zeta_{\Gamma}}{\mathrm{d} t}=P\left(\zeta_{\Gamma}\right)
$$

Using an integration step $\Delta t$ and assuming that at time $t_{n}$ the vortex position $\zeta_{\Gamma}^{n}$ is known, the position $\zeta_{\Gamma}^{n+1}$ at time $t_{n+1}=t_{n}+\Delta t$ is calculated by using:

$$
\zeta_{\Gamma}^{n+1}=\zeta_{\Gamma}^{n}+\frac{1}{6}\left(k_{1}+2 k_{2}+2 k_{3}+k_{4}\right)
$$

where: 


$$
\begin{gathered}
k_{1}=\Delta t P\left(\zeta_{\Gamma}^{n}\right) \\
k_{2}=\Delta t P\left(\zeta_{\Gamma}^{n}+\frac{k_{1}}{2}\right) \\
k_{3}=\Delta t P\left(\zeta_{\Gamma}^{n}+\frac{k_{2}}{2}\right) \\
k_{4}=\Delta t P\left(\zeta_{\Gamma}^{n}+k_{3}\right) .
\end{gathered}
$$

Using the proposed integration scheme, we can at each time step determine for a given position $\zeta_{\Gamma}$ in the $\zeta$-plane the new vortex position $z_{\Gamma}$ in the $z$-plane by using an explicit conformal mapping e.g. equation (3.13) or equation (3.17). As a test the path of a vortex around the edge at $x=0$ of a semi-infinite plane $(x<0)$ is considered. The conformal mapping is

$$
z=\zeta^{2}
$$

i.e.

$$
\begin{aligned}
\frac{\mathrm{d} z}{\mathrm{~d} \zeta} & =2 \zeta \\
\frac{\mathrm{d}^{2} z}{\mathrm{~d} \zeta^{2}} & =2 .
\end{aligned}
$$

We have

$$
F_{\Gamma}=-\frac{i}{2 \pi} \Gamma \ln \left(\frac{\zeta-\zeta_{\Gamma}}{\zeta+\zeta_{\Gamma}^{*}}\right)
$$

Crighton (Crighton 1972) provides the analytical solution for the path of a vortex passing at $t=0$ through $z=L$. Where $L$ is the distance from the edge in polar coordinates

$$
r=L \sqrt{1-\left(\frac{\Gamma t}{8 \pi L^{2}}\right)^{2}}
$$

and

$$
\theta=2 \arctan \left(-\frac{\Gamma t}{8 \pi L^{2}}\right) .
$$

Viz. we have

$$
z_{\Gamma}=r(\cos \theta+\mathrm{i} \sin \theta)
$$

Figure E.1 compares the numerical solution with the analytical solution. The relative difference in the vortex position is of the order of the machine accuracy $10^{-15}$. 


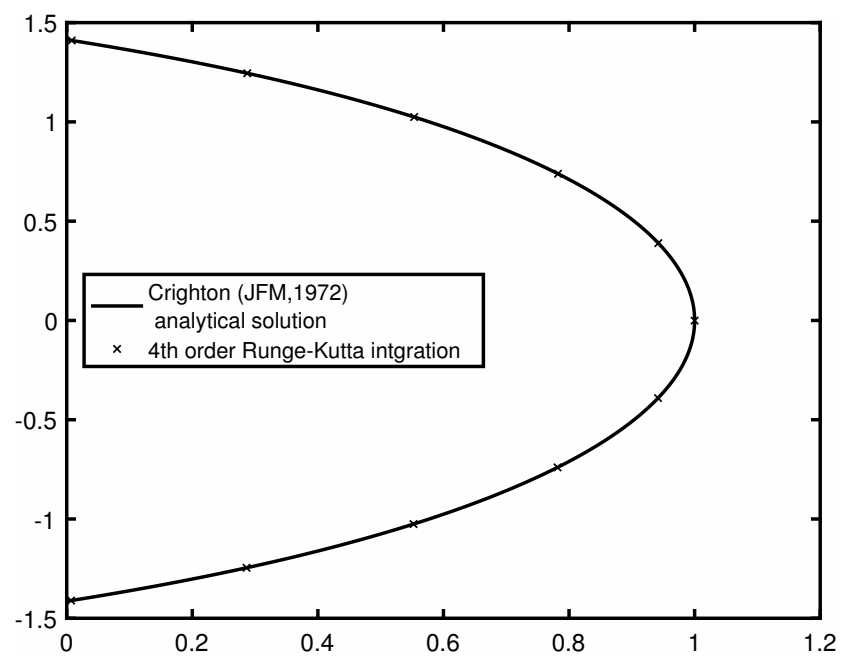

Figure E.1: Vortex path around a semi-infinite plane (around the negative real axis): comparison of 4 th order Runge-Kutta integration with analytical solution of Crighton (Crighton 1972). 



\section{Appendix F}

\section{Derivation of equation used to determine the order of accuracy}

In the following the equation used to determine the order of accuracy of numerical simulations is provided. When one has verified that the calculated order of accuracy corresponds, within a satisfactory degree of accuracy, to the order of the discretization method used in simulations one ascertains the results to be grid independent (Hulshoff 2016a).

Assuming one uses a $n^{\text {th }}$ order accurate numerical method, viz with a numerical error

$$
\epsilon_{i}=A \Delta x_{i}^{n} .
$$

The difference between the computed quantity of interest on a coarse mesh $p_{i+1}^{\prime}$ and the exact solution $p_{\infty}^{\prime}$ is then

$$
p_{i}^{\prime}-p_{\infty}^{\prime}=A \Delta x_{i}^{n}
$$

for a finer mesh $p_{i+1}^{\prime}$ this is

$$
p_{i+1}^{\prime}-p_{\infty}^{\prime}=A \Delta x_{i+1}^{n} .
$$

The difference in computed value between the coarse mesh and fine mesh is

$$
p_{i}^{\prime}-p_{i+1}^{\prime}=A\left(\frac{\Delta x_{i}^{n}}{\Delta x_{i+1}^{n}}-1\right) \Delta x_{i+1}^{n} .
$$

The difference between the computed value of the fine mesh $p_{i+1}^{\prime}$ and the value obtained with an even finer mesh $p_{i+2}^{\prime}$

$$
p_{i+1}^{\prime}-p_{i+2}^{\prime}=A\left(\frac{\Delta x_{i+1}^{n}}{\Delta x_{i+2}^{n}}-1\right) \Delta x_{i+2}^{n} .
$$


Combining Eq. F.4 and (F.5), one obtaines

$$
\frac{p_{i}^{\prime}-p_{i+1}^{\prime}}{p_{i+1}^{\prime}-p_{i+2}^{\prime}}=\left(\frac{\Delta x_{i+1}}{\Delta x_{i+2}}\right)^{n}
$$

Solving for $n$, yields

$$
n=\frac{\ln \left(\frac{p_{i}^{\prime}-p_{i+1}^{\prime}}{p_{i+1}^{\prime}-p_{i+2}^{\prime}}\right)}{\ln \left(\frac{\Delta x_{i+1}}{\Delta x_{i+2}}\right)}
$$

where $\frac{\Delta x_{i+1}}{\Delta x_{i+2}}$ is the ratio of refinement between meshes. Fixing the ratio $\frac{\Delta x_{i+1}}{\Delta x_{i+2}}$, one can use Eq. F.7 to verify that the solutions obtained on three different mesh are on the convergence domain. I.e. one calculates the observed order of accuracy. N.b. this observed order of accuracy should be close to the order of the numerical method used.

For the vortex-nozzle and entropy spot nozzle interaction this observed order of accuracy was found to be 1.8. Simulation were carried out using a second order TVD Roe method with a van Leer limiter, as such the observed order of accuracy of 1.8 is satisfactory. 


\section{Appendix G}

\section{Vortex passing through a nozzle with cavity}

In this appendix a figure similar to Fig. 4.15 is provided. It shows that, as is the case without a nozzle cavity (Fig. 4.15), sound is produced when the vortex is on approach to the nozzle with cavity. Note the presence of the second channel-cavity mode on the signal, see chapter 4.7 for an explanation. 


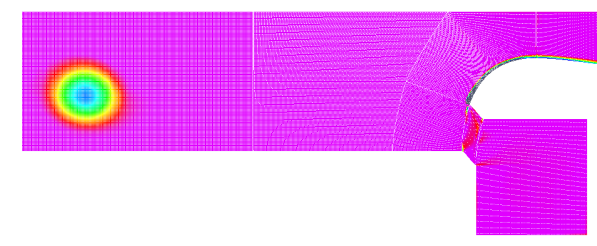

(a) $\left(t-t_{\text {travel }}\right) U / S_{1} \simeq 4.0$

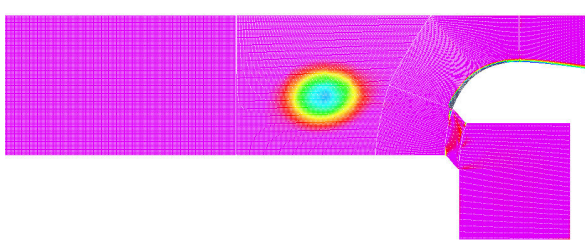

(c) $\left(t-t_{\text {travel }}\right) U / S_{1} \simeq 5.9$

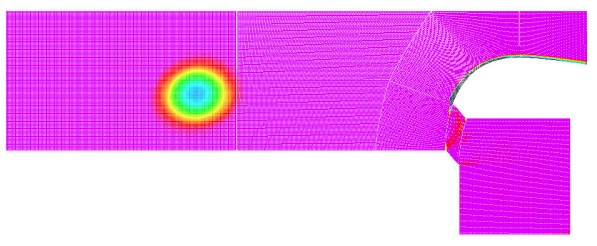

(b) $\left(t-t_{\text {travel }}\right) U / S_{1} \simeq 4.9$

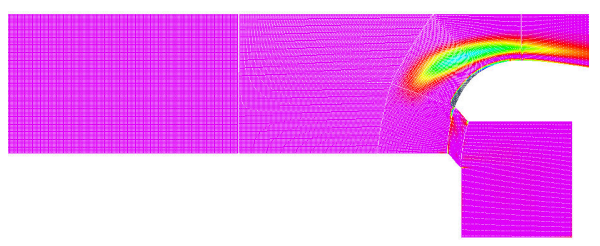

(d) $\left(t-t_{\text {travel }}\right) U / S_{1} \simeq 6.9$

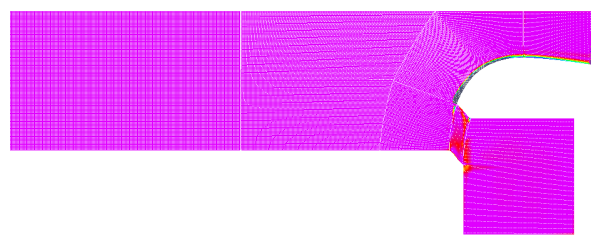

(e) $\left(t-t_{\text {travel }}\right) U / S_{1} \simeq 7.9$

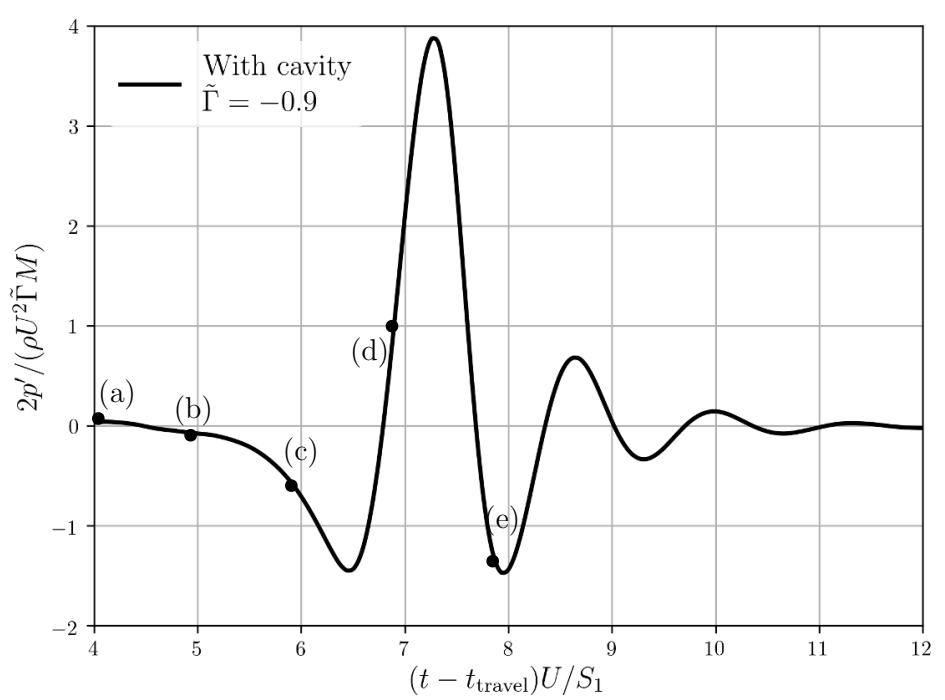

(f) Recorded signal at upstream probe

Figure G.1: Vortex of $\tilde{\Gamma}=-0.9$ and $R_{\Gamma}=0.3 S_{1}$ passing through a nozzle with cavity. The vortex was released from $(-2,0.4) S_{1}$ i.e. the vortex release height used was $h=0.4 S_{1}$. 


\section{Appendix $\mathrm{H}$}

\section{Acoustic loss models}

This appendix contains a summary of the loss models used for the model. More detail on these models can be found in (Hirschberg et al. 2016).

\section{H.1 Nozzle radiation}

The losses due to the radiation of the plane waves at the nozzle is estimated by using the quasi-steady isentropic theory of Marbel and Candel (Marble and Candel 1977). The key idea is that in this approximation the Mach number at the nozzle inlet is fixed by the geometry as long as the nozzle remains chocked. This implies for the fluctuations $c^{\prime}$ in speed of sound $c$ at the nozzle inlet:

$$
\frac{c^{\prime}}{c}=\frac{u^{\prime}}{U} .
$$

Using the isentropic gas relations for a perfect gas, combined with $p^{\prime}=c^{2} \rho^{\prime}=$ $\left(p^{+}+p^{-}\right)$and $u^{\prime}=\left(p^{+}-p^{-}\right) /\left(\rho_{0} c\right)$, one finds the pressure reflection coefficient:

$$
R_{\mathrm{nozzle}}=\frac{p^{-}}{p^{+}}=\frac{1-\frac{\gamma-1}{2} M}{1+\frac{\gamma-1}{2} M} .
$$

This is the result of Marble and Candel (Marble and Candel 1977). Thus the stagnation enthalpy reflection coefficient is

$$
\frac{B^{-}}{B^{+}}=R_{\text {nozzle }} \frac{1-M}{1+M}
$$

and the time averaged power losses $\left\langle\mathcal{P}_{\text {nozzle }}\right\rangle$ due to this radiation are given by

$$
\left\langle\mathcal{P}_{\text {nozzle }}\right\rangle=\frac{\rho_{0}\left|b_{n}\right|^{2}}{8 c}\left[1-R_{\text {nozzle }}^{2}\left(\frac{1-M}{1+M}\right)^{2}\right] \pi r_{p}^{2},
$$


where it is assumed that $\left|B^{+}\right|=\left|B^{-}\right|=\left|b_{n}\right| / 2$ in accordance with the assumption that the acoustic field is dominated by a standing wave, described by Eq. (5.6). Alternatively, based on Eq. (5.12), the nozzle radiation can be written in terms of $\gamma_{\text {nozzle }}$ as

$$
\left\langle\mathcal{P}_{\text {nozzle }}\right\rangle=\frac{\gamma_{\text {nozzle }}}{2} \omega^{2}\left|b_{n}\right|^{2},
$$

from which the nozzle damping coefficient is deduced

$$
\gamma_{\text {nozzle }}=\frac{\rho_{0} \pi r_{p}^{2}}{4 \omega^{2} c}\left[1-R_{\text {nozzle }}^{2}\left(\frac{1-M}{1+M}\right)^{2}\right]
$$

\section{H.2 Vortex shedding at the orifice}

Due to flow separation at the sharp edges of the orifice a free jet will be formed downstream of the orifice with a smaller radius $r_{\text {jet }}$ than the orifice radius $r_{o}$. The ratio $\left(r_{\text {jet }} / r_{o}\right)^{2} \equiv \Upsilon$ is called the vena contracta factor. It is a function of $r_{o} / r_{p}$. Empirical data by Gilbarg (Gilbarg 1960) is used to determine this vena contracta factor in Section 5.2. Neglecting the pressure recovery downstream of the orifice one has a pressure in the free jet equal to the downstream acoustic pressure. This implies a loss of total acoustical enthalpy $B^{\prime}$ across the orifice given, for a quasi-steady flow, by

$$
\text { section } \Delta B_{\text {orifice }}^{\prime}=u_{\text {jet }}^{\prime} U_{\text {jet }}-u^{\prime} U=u^{\prime} U\left[\left(\frac{r_{p}}{r_{\text {jet }}}\right)^{4}-1\right]
$$

where $u^{\prime} U$ is evaluated just upstream of the orifice. Terms of order $M^{2}$ are neglected and it is assumed that the surface averaged velocities $u^{\prime}$ and $U$ are equal upstream and downstream of the orifice.

The corresponding acoustic power losses are given by

$$
\left\langle\mathcal{P}_{\text {orifice }}\right\rangle=\left\langle m^{\prime} \Delta B_{\text {orifice }}^{\prime}\right\rangle \pi r_{p}^{2}
$$

From which, in a similar way as for the nozzle radiation, one finds:

$$
\gamma_{\text {orifice }}=\frac{\rho_{0} \pi r_{p}^{2}}{\omega^{2} c}\left(\frac{\omega_{n}}{\omega}\right) M\left[\left(\frac{r_{p}}{r_{\text {jet }}}\right)^{4}-1\right] \sin ^{2}\left(k_{n} x_{o}\right),
$$

where the orifice position is $x_{o}=L_{o}+V_{c} / \pi r_{p}^{2}$. 


\section{H.3 Viscous damping at the porous wall}

A quasi-steady isothermal flow through the porous wall is assumed. By continuity the mass flux $m=\rho_{0} u$ remains independent of the position $x$ in the porous wall. As for $M^{2}<<1$ the temperature remains uniform in the wall and the viscosity $\mu$ of the air is a function of the temperature only (ideal gas), one finds that the Reynolds number $R e_{d}=\rho_{0} u \bar{d} / \mu$ based on the characteristic pore width $\bar{d}$ is constant in the porous wall. The pressure gradient in the wall is determined by a friction coefficient $C_{D}$, which is assumed to be a function of $R e_{d}$ only (Schmidt 2015) :

$$
\frac{\mathrm{d} p}{\mathrm{~d} x}=-\frac{\left(\rho_{0} u\right)^{2}}{\rho_{0} \bar{d}} C_{D}\left(R e_{d}\right)
$$

As $\rho_{0}=p /(R T)$ with $T$ the temperature and $R$ the specific gas constant one finds by integration $p_{r}^{2}-p^{2}=C\left(\rho_{0} u\right)^{2}$, with $C$ a constant. Neglecting the acoustic perturbations in the high pressure reservoir and using $\rho^{\prime} / \rho_{0}=p^{\prime} / p$ for isothermal flow, one finds:

$$
\frac{p^{\prime}}{p}=\left[1-\left(\frac{p_{r}}{p}\right)^{2}\right]\left(\frac{p^{\prime}}{p}+\frac{u^{\prime}}{U}\right)
$$

Using $p^{\prime}=p^{+}+p^{-}, \rho^{\prime}=p^{\prime} / c^{2}, u^{\prime}=\left(p^{+}-p^{-}\right) /\left(\rho_{0} c\right)$, after some algebra, one finds

$$
\gamma_{\text {porous }}=\frac{\rho_{0} \pi r_{p}^{2}}{4 \omega^{2} c}\left[1-R_{\text {porous }}^{2} \frac{(1+M)^{2}}{(1-M)^{2}}\right]
$$

with

$$
R_{\text {porous }}=\frac{p^{+}}{p^{-}}=\frac{\left(p_{r} / p\right)^{2}(1-\gamma M)-1}{\left(p_{r} / p\right)^{2}(1+\gamma M)-1} .
$$





\section{Appendix I}

\section{Euler Internal Aeroacoustics code}

In this appendix a brief description of the Euler internal Aeroacoustics code (EIA), copied form the EIA manual (Hulshoff 2016b) is provided.

\section{I.1 Euler equations}

In EIA (Euler internal Aeroacoustics code) the flow is modeled using Euler equations, which describe the conservation of mass, momentum and energy for a compressible non-heat conducting inviscid fluid. The Euler equations may be written in integral form for an arbitrary control volume $V$, with enclosing surface $S$ and outer normal n (Fig. I.2) as (Hulshoff 2016b):

$$
\frac{\mathrm{d}}{\mathrm{d} t} \int_{V} \mathbf{W} \mathrm{d}^{3} x+\int_{S} \mathbf{F} \cdot \mathbf{n} \mathrm{d}^{2} x=\int_{V} \boldsymbol{\Phi} \mathrm{d}^{3} x
$$

where $\mathbf{W}$ is the vector of conserved quantities

$$
\mathbf{W}=\left[\begin{array}{c}
\rho \\
\rho \mathbf{u} \\
\rho e_{\text {total }}
\end{array}\right]
$$

with $\rho$ the mass density, $\mathbf{u}=(u, v)$ is the velocity vector (two dimensional planar flow), and $e_{\text {total }}$ is the total energy per unit mass. The normal component of the fluxes through the surface of the control volume is:

$$
\mathbf{F} \cdot \mathbf{n}=\left[\begin{array}{c}
\rho(\mathbf{u} \cdot \mathbf{n}) \\
\rho \mathbf{u}(\mathbf{u} \cdot \mathbf{n})+p \mathbf{n} \\
\left(\rho e_{\text {total }}+p\right)(\mathbf{u} \cdot \mathbf{n})
\end{array}\right]
$$




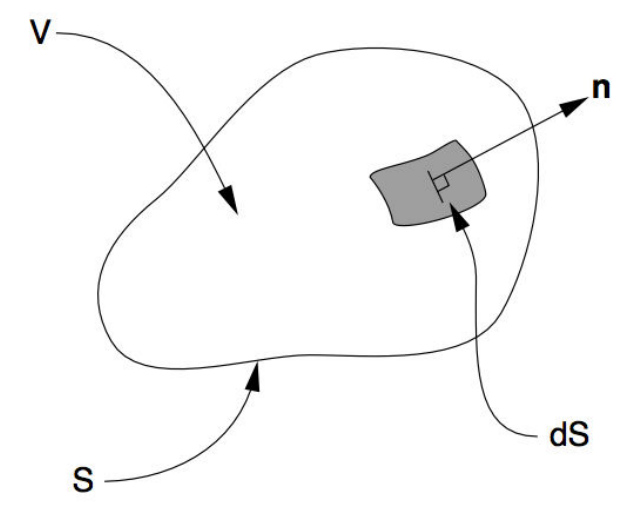

Figure I.1: Arbitrary control volume, drawing taken from (Hulshoff 2016b).

where $p$ is the static pressure. In EIA, it is assumed that the fluid behaves as a calorically ideal gas with constant specific heat (Hulshoff 2016b). The sources are in vector $\boldsymbol{\Phi}$ :

$$
\boldsymbol{\Phi}=\left[\begin{array}{c}
0 \\
\mathbf{f} \\
\mathbf{f} \cdot \mathbf{u}+\dot{Q}
\end{array}\right]
$$

f is an external force density (optional), $\dot{Q}$ is the rate of heat produced per unit volume by external agencies (optional).

\section{I.2 Advantages and disadvantages applying Euler equa- tions}

Application of the Euler equations to internal Aeroacoustics has several main advantages (Hulshoff 2016b):

- As all inviscid compressibility effects are included, their application is not limited to low-Mach number or low-frequency conditions.

- When solved in their integral form, they admit discontinuities such as shock waves.

- The interaction and propagation of acoustic waves is directly represented without the use of additional models.

- Comparison of accurate Euler solutions with experimental results can be used to unambiguously evaluate the relative importance of inviscid and viscous effects.

Since viscous effects are neglected this presents some limitations, mainly:

- Flow features strongly affected by viscous effects, such as separations from a smooth surface cannot be represented. 


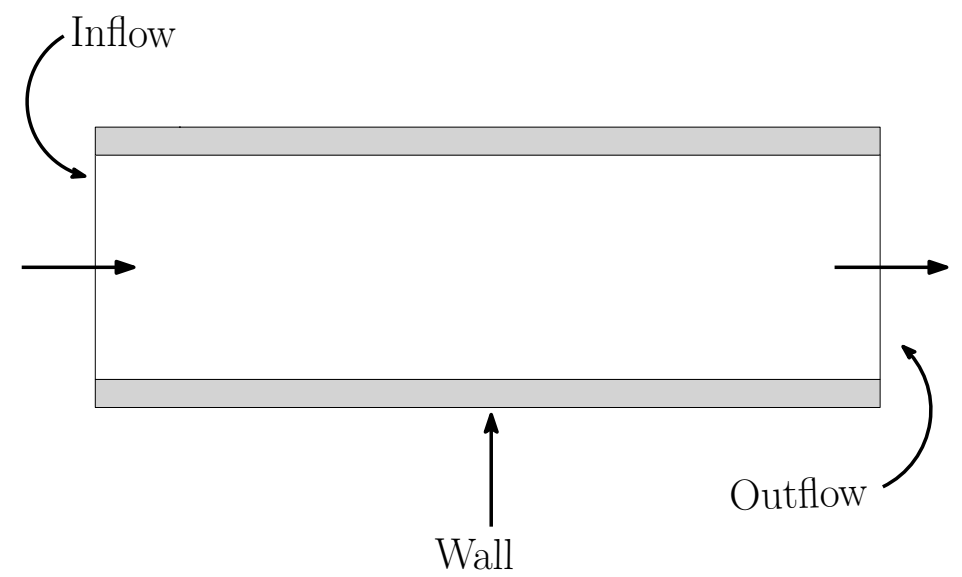

Figure I.2: Example of numerical domain which is a truncated region of the physical domain with Inflow/Outflow and Wall boundary conditions.

- Noise due to turbulence cannot be predicted ${ }^{1}$.

Although it is possible to solve the Navier-Stokes equations to overcome the first of these deficiencies. It would present a significantly greater computational effort than for the Euler solutions. This is due to the order of magnitude increase in grid resolution necessary for the accurate computation of viscous forces at higher Reynolds numbers. This requires both an increase in work per time step and a larger number of time steps due to the increased numerical stiffness (Hulshoff 2016b).

Overcoming the second deficiency, the computation of noise due to turbulence, has even greater resolution requirements. Particular computations of this type require application of approximate models, the effectiveness of which remains a topic of research (Hulshoff 2016b; Colonius and Lele 2004).

\section{I.3 Boundary conditions}

The solution of the Euler equations is tied to a particular physical situation through specification of the boundary conditions. This is not necessarily straight-forward, as the directions of information propagation inherent in the Euler equations must be respected to ensure well-posed solutions. This means that only certain combinations of boundary conditions are valid, placing limits on what may be directly specified by the user. A number of different types of boundary are be applied using EIA. These are

\footnotetext{
${ }^{1}$ As a matter of fact, a two dimensional planar model as considered here cannot be used to describe turbulence.
} 
- Inflow/Outflow conditions,

- Wall/Symmerty conditions.

An example of the application of these boundary conditions is shown in Fig. I.2

\section{I.3.1 Inflow/Outflow conditions}

To limit computational expense, the numerical domain is defined as a truncated region of the physical domain. This requires the definition of artificial inflow and outflow boundaries which allow the flow to enter and leave the numerical domain. When applying conditions on inflow and outflow boundaries, care must be taken to ensure that correct directions of information propagation inherent in the Euler equations are preserved.

The Euler equations are a hyperbolic system which express the convection of entropic, vortical and acoustic waves (Hulshoff 2016b).

Consider the case of plane waves propagating normal to a boundary surface. This can be illustrated using a one-dimensional $x-t$ diagram. The case for a subsonic outflow is shown in Fig. I.3. There are four characteristics in Fig. I.3:

1. right traveling acoustic wave (coarsely dashed line) with convection speed $u+c$,

2. entropy wave (dash dotted line) with convection speed $u$,

3. vortical waves (dash dotted line) with convection speed $u$,

4. left traveling acoustic wave (finely dashed line) with convection speed $u-c$.

To determine the state at point $\mathrm{A}$ (where these characteristics meet in Fig. I.3), information is required from each of the characteristics. For the boundary considered in Fig. I.3, the $u+c$ and the two $u$ characteristics are all emanating from within the numerical domain. The local strength at A of the entropic, vortical and right traveling acoustic waves is thus determined by the evolving numerical solution. For a subsonic flow $u<c$, the left traveling wave is emanating from outside the numerical domain, hence, the strength of the left traveling acoustic wave must be determined by a physical model of what occurs outside of the numerical domain, which the user must to specify.

Examples of these user specified models include:

- no incoming waves (anechoic exit ${ }^{2} p_{2}^{-}=0$ ),

- an acoustic wave which maintains constant pressure (a quasi-steady model for an exit into an infinite reservoir: $\left.p_{2}^{+}+p_{2}^{-}=0\right)$,

- a left traveling harmonic acoustic wave $\left(p_{2}^{-}=\mathfrak{A} \sin \left(\omega\left(t-\frac{x}{u-c}\right)\right)\right)$.

It should be noted that although a system for four unknown variables is being solved, the user is in fact only free to specify one physical condition in the case of subsonic outflow (Fig. I.3(b)). The situation for each of the subsonic

\footnotetext{
${ }^{2}$ The subscript 2 indicates that the left traveling (- superscript) acoustic wave $p_{2}^{-}$comes from outside of the numerical domain.
} 


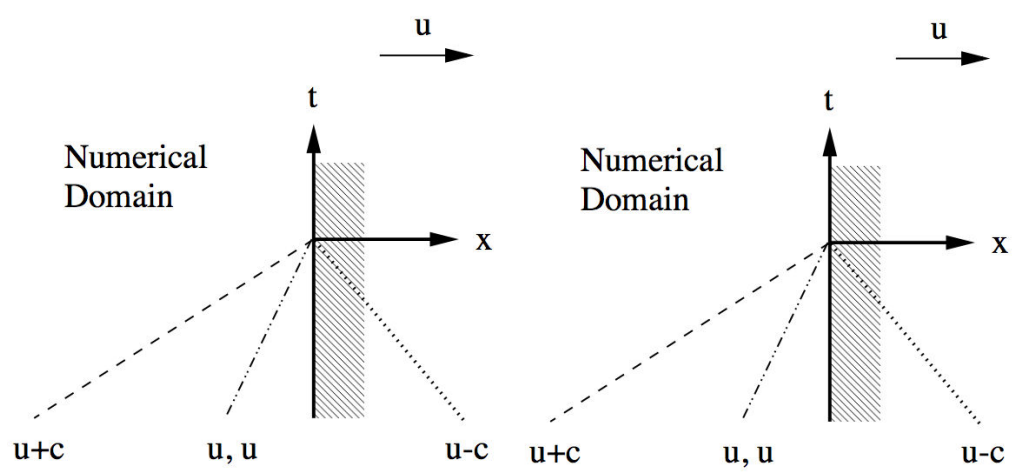

(a) One dimensional space time (b) One dimensional space time $(x-$ $(x-t)$ diagram for the case of a $t$ ) diagram for the case of a subsubsonic inflow boundary. The $u$, sonic outflow boundary. Only the $u$ and $u-c$ characteristics enter the $u-c$ characteristic enters the numernumerical domain, thus three phys- ical domain, thus one physical paramical parameters can be user speci- eters can be user specified. Figure fied. Figure taken from (Hulshoff taken from (Hulshoff 2016b). 2016b).

Figure I.3: Subsonic inflow and outflow cases.

inflow/outflow is summarized in Fig. I.3. The supersonic inflow/outflow cases are summarized in Fig. I.4.

\section{I.3.2 Wall/Symmetry conditions}

A wall condition is one which defines a surface across which the normal velocity is zero viz. $\mathbf{u} \cdot \mathbf{n}=0$. Such a condition may be used to define a channel boundary or a symmetry plane used to reduce the size of the numerical domain for symmetrical problems (Fig. I.5).

For a wall/symmetry boundary condition only one characteristic enters from outside the numerical domain. The physical condition that $\mathbf{u} \cdot \mathbf{n}=0$ be maintained is thus the only one that can be user defined. It is thus not possible for to specify additional conditions at the wall such as the zero tagential velocity which would be present in the viscous flow case.

\section{I.4 Numerical techniques}

The numerical solution technique employed by EIA makes use of separate space and time discretization. The system of integral governing equations (Eq. (I.1)) is first discretised in space using a finite volume method in the interior of the numerical domain. This results in a large system of ordinary differential equations for the time rates of change of state variables in each computational 


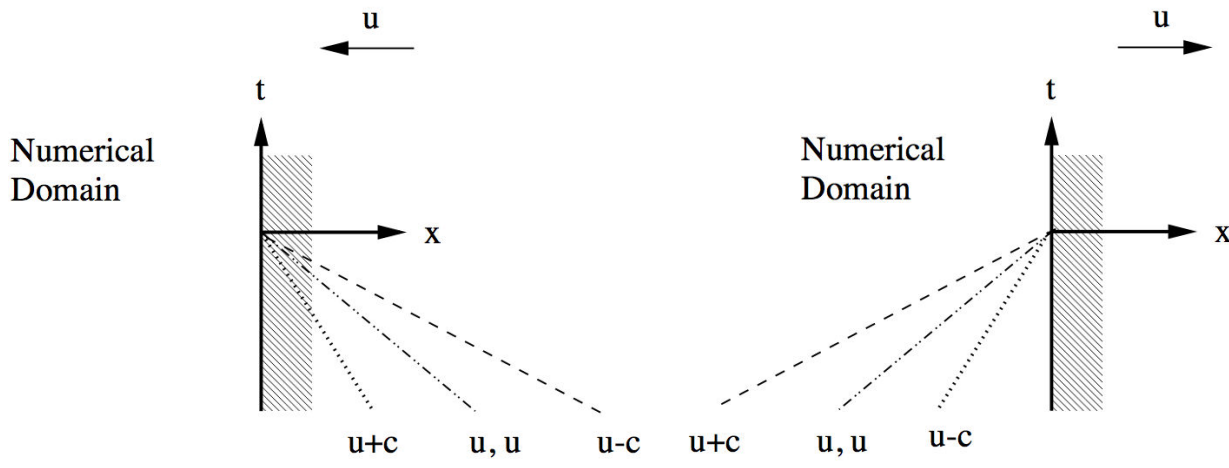

(a) One dimensional space time $(x-t)$ diagram (b) One dimensional space time $(x-t)$ difor the case of a supersonic inflow boundary. agram for the case of a supersonic outAll the characteristics enter the numerical do- flow boundary. None of the characterismain, thus four physical parameters can be user tics enters the numerical domain, thus no specified. Figure taken from (Hulshoff 2016b). physical parameters can be user specified.

Figure taken from (Hulshoff 2016b).

Figure I.4: Supersonic inflow and outflow cases.

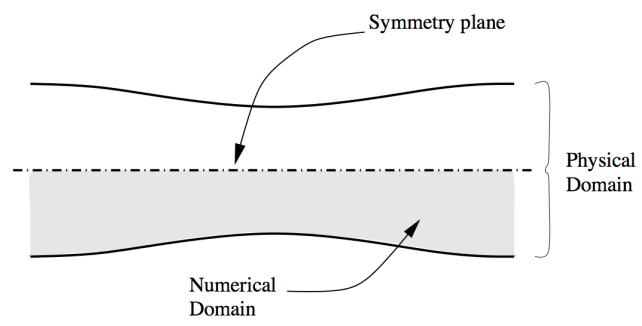

Figure I.5: Example of a symmetrical problem in which symmetry boundary conditions are used to reduce the size of the numerical domain. Figure taken from (Hulshoff 2016b). 


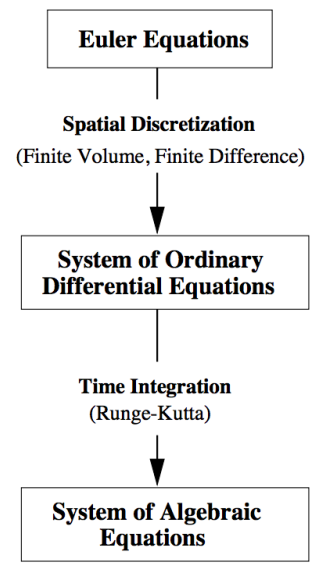

Figure I.6: Overview of the numerical discretization technique. Figure taken from (Hulshoff 2016b).
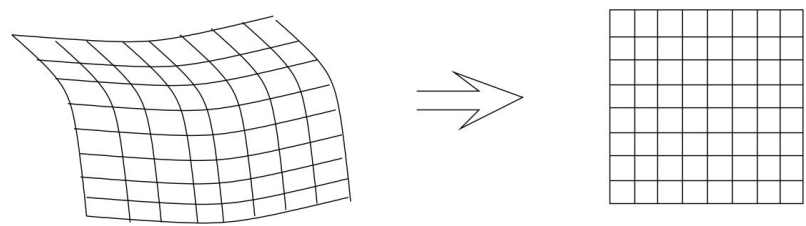

Figure I.7: Structured grid block (left) and mapping to a Cartesian domain (right). Figure taken from (Hulshoff 2016b).

cell. These equations are in turn transformed to a fully discrete algebraic system for the unknowns at each time step by the application of the Runge-Kutta time integration method. In Fig. I.6 an overview of the discretization technique is shown.

In this section a brief review of how these numerical solution techniques are implemented is given.

\section{I.4.1 Spatial discretization}

\section{I.4.1.1 Grid definition}

Before applying a spatial discretization procedure, it is necessary to define a grid for the numerical domain. EIA employs multiple structured grid blocks for this purpose. A structured grid block consists of families of regular and non crossing coordinate lines, which permit the mapping of the grid to a simplified Cartesian domain (Fig. I.7). 


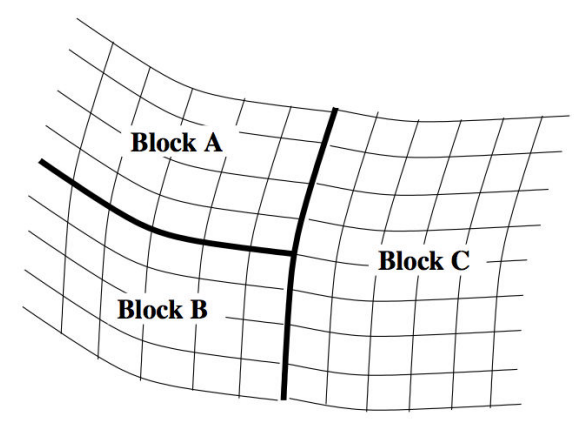

(a) Two neighboring blocks (left) bordering a single block side (right).

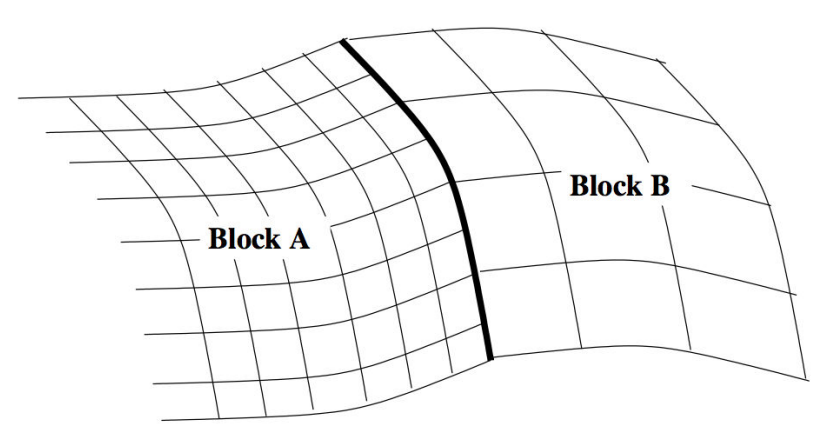

(b) Two neighboring blocks with different numbers of cells.

Figure I.8: Examples of neighboring blocks allowed in EIA, Figures taken from (Hulshoff 2016b).

EIA allows the use of multiple structured grid blocks connected with arbitrary orientations. Each side of a structured block may border more than one neighboring block, see Fig. I.8(a). The number of cells across a block interface may also differ, provided that the number of finer cells which border each coarse cell remains an integer constant, as shown in Fig. I.8(b).

\section{I.4.1.2 Interior discretization}

The Euler equations are discretised in EIA in the interior of the numerical domain using a cell centred finite volume technique. This is a conservative discretization technique which allows discontinuities such as shocks to be directly computed as part of the solution. The technique applies the integral form of the conservation laws (Eq. (I.1)) to each grid cell in the computational domain, as shown in Fig. I.9.

For a grid of $N$ cells this results in a system of $4 N$ ordinary differential equations 


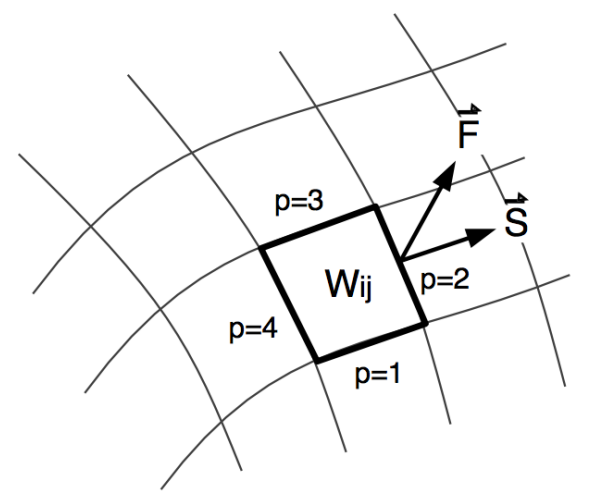

Figure I.9: Cell centred finite volume discretization. Figure taken from (Hulshoff 2016b).

for the time derivatives of the volume averaged state variables in each cell:

$$
\frac{\mathrm{d}}{\mathrm{d} t} W_{i j} V_{i j}+\sum_{p=1}^{P} \mathbf{F}_{p} \cdot \mathbf{S}_{p}=0
$$

here $W_{i j}$ is the volume averaged state variable, $V_{i j}$ is the cell volume, $\mathrm{P}$ is the number of sides which define the faces of the cell, $P=4$ in Fig. I.9., $\mathbf{F}_{p}$ is the flux of the considered variables on face $p$, and $\mathbf{S}_{p}$ is the area vector on face $p$ While the unknowns $W_{i j}$ are associated with the cell centers, the fluxes are defined at the faces and must be reconstructed from the surrounding state variables. Finite volume methods differ on how this is done. If simple averaging is used, a method with zero dissipation for linear systems will be obtained. In the nonlinear case of Euler equations, this is insufficient to ensure numerical stability.

In EIA $\mathbf{F}_{p}$ can be evaluated using an upwind scheme based on Roe's approximate Riemann solver. The implementation is total variation diminishing (TVD), which minimises oscillatory behaviour, making the scheme more robust. The Roe-TVD scheme also provides more accurate predictions of solution jumps across discontinuities. Its disadvantage is its relatively high computational cost per time step. The behaviour of the Roe TVD scheme is controlled by the choice of limiter function. When no limiter function is used a first-order upwind scheme is obtained. The remaining limiter functions (minmod, van Leer, superbee) all produce second-order accurate schemes, with minmod being the most dissipative and superbee the least.

The results of vortex nozzle interaction simulations described later in this text were obtained with a second order Roe-TVD scheme with a van Leer limiter. 


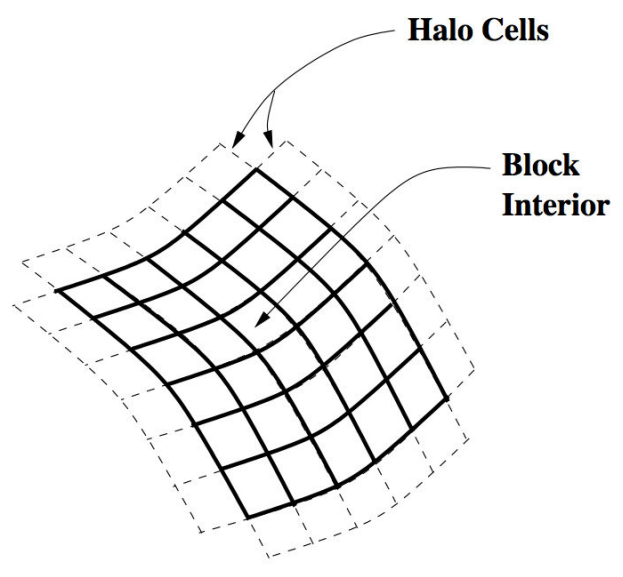

Figure I.10: Halo cells used for the enforcement of boundary conditions. Figure taken from (Hulshoff 2016b).

\section{I.4.1.3 Boundary discretization}

In EIA, boundary conditions are applied by specifying the state of halo cells just outside the numerical domain, Fig. I.10, and including these cells in the normal interior discretization procedure.

For wall boundaries, the halo cell states are determined from the evolving numerical solution in a manner which ensures satisfaction of $\mathbf{v} \cdot \mathbf{n}=0$ at the wall.

In the case of inflow and outflow boundaries, halo cell states are determined using a finite discretization of the characteristic form of the Euler equations (Hulshoff 2016b). When discretizing an inflow or outflow boundary, only the characteristic relations which describe the information propagating from within the numerical domain are retained. The remaining necessary equations to determine the boundary state are provided by the physical model of the region outside the numerical domain.

The most simple of these models sets the incoming acoustic waves to zero or specifies their their magnitudes in time. There also exists conditions which specify the acoustic waves indirectly through the specification of primitive such as pressure and velocity.

Finally there exists a class of "soft constant" boundary condition, which approximately maintain a constant state, by slowly feeding in acoustic waves proportional to the difference between the instantaneous state and the desired one. 


\section{I.4.2 Time integration}

The time integration method employed in EIA is based on a multi-stage low storage Runge-Kutta technique. For the $K$-stage integration of a single variable it can be expressed as:

$$
\begin{aligned}
w_{n+1}^{1} & =w_{n}+\alpha^{1} \Delta t\left[\left(\frac{\partial w_{n}}{\partial t}\right)_{c}+\left(\frac{\partial w_{n}}{\partial t}\right)_{d}\right]_{c} \\
w_{n+1}^{2} & =w_{n}+\alpha^{2} \Delta t\left[\left(\frac{\partial w_{n+1}^{1}}{\partial t}\right)_{c}+\left(\frac{\partial w_{n+1}^{1}}{\partial t}\right)_{d}\right] \\
\vdots & \\
w_{n+1}^{k} & =w_{n}+\alpha^{2} \Delta t\left[\left(\frac{\partial w_{n+1}^{k-1}}{\partial t}\right)_{c}+\left(\frac{\partial w_{n+1}^{M}}{\partial t}\right)_{d}\right] \\
\vdots & \\
w_{n+1} & =w_{n}+\alpha^{K} \Delta t\left[\left(\frac{\partial w_{n+1}^{K-1}}{\partial t}\right)_{c}+\left(\frac{\partial w_{n+1}^{M}}{\partial t}\right)_{d}\right]
\end{aligned}
$$

here the subscript $n$ denotes the current time step, and the superscript denotes the Runge-Kutta stage. The time derivative operator is broken into a central component and a component due to artificial dissipation. The artificial dissipation contribution remains fixed after $M$ stages, where $M \leq K$. The $\alpha$ coefficients can be chosen to control the stability and accuracy of the time integration. In most applications, the coefficients are chosen to maintain second-order accuracy in time.

The vortex nozzle interaction results latter described in this text were obtained using a five stage Runge-Kutta scheme. 



\section{Appendix J}

\section{Entropy spot generation}

To study entropy spot-nozzle interaction a entropy spot needs to be generated. This is done on a entropy spot-less base flow, the establishment of which is described in subsection 4.6.3. The stationary base flow is used to initialize the domain in which entropy spot-nozzle interactions are carried out. An example of a mesh, without nozzle cavity, on which entropy spot-nozzle interaction simulations are carried out is shown in Fig. J.1.

The boundary conditions (Hulshoff 2016b) used in entropy spot-nozzle interaction simulations are as follows.

- An acoustically non-reflective condition on the upstream wall (on the lefthand side of Fig. J.1) this boundary condition:

- mimics a connection to an infinite upstream channel,

- it maintains the local average inflow velocity, density and sound speed imposed by the base flow as an initial condition.

- Wall boundary conditions on the lower and upper walls of the channel.

- Connection boundary conditions on all the interfaces connecting the constituent blocks of the channel.

- Non-reflective boundary condition on the downstream end boundary.

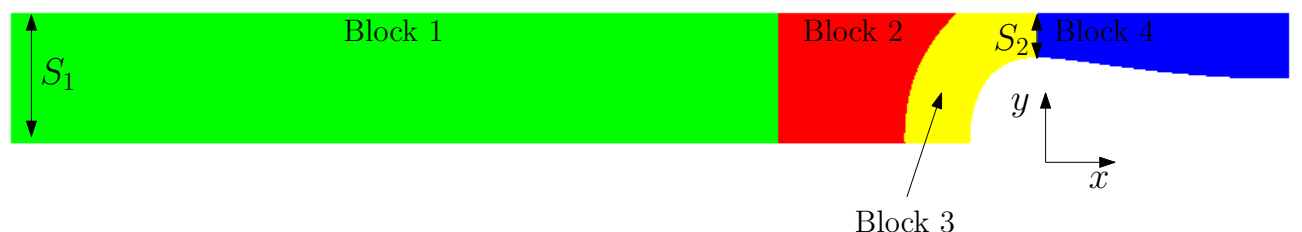

Figure J.1: Example of mesh for entropy spot-nozzle interaction. For a nozzle without a nozzle cavity. 


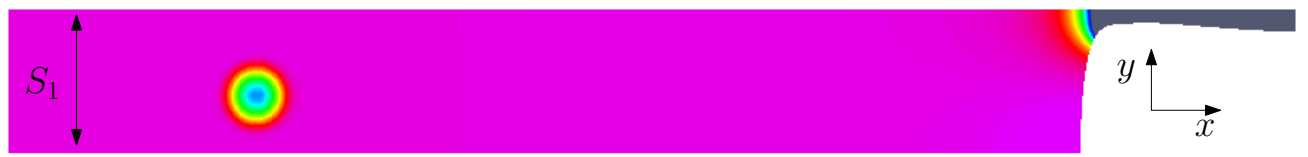

(a) $t U / S_{1} \simeq 0.64$

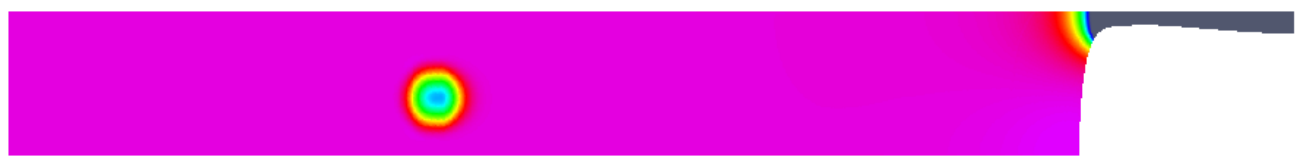

(b) $t U / S_{1} \simeq 1.9$

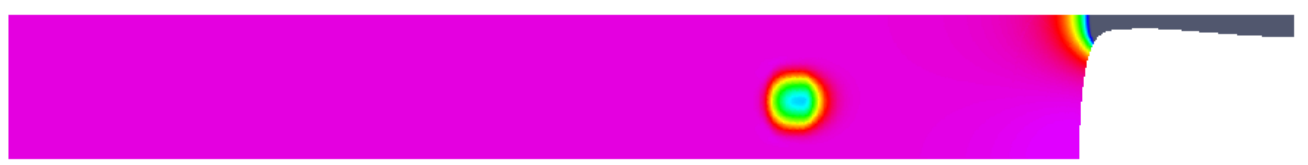

(c) $t U / S_{1} \simeq 4.5$

Figure J.2: Snapshots of entropy spot during and after the generation process. The results shown here were obtained with upstream Mach number $M=0.053$, release height $h=0.4 S_{1}$, entropy-spot-core radius $R_{s}=0.3 S_{1}$. The generation time interval is $0 \leq t / U / S_{1} \leq 0.80$. In subfigure (a) the entropy spot is still being generated, whilst in subfigures $(b)$ and $(c)$ a fully mature entropy spot with maximum density deviation $(\delta \rho / \rho)_{\max }=-0.03$ is shown.

A few snapshots of a entropy spot during the generation process are shown in Fig. J.2. The snapshots were obtained with upstream Mach number $M=$ 0.053 , release height $h=0.4 S_{1}$, entropy-spot-core radius $R_{s}=0.3 S_{1}$. The generation process of this entropy spot was started a distance $S_{1}$ from the upstream wall, at $\mathbf{x}_{0}=\left(x_{0}, y_{0}\right)=(-6.5,0.4) S_{1}$.

To generate a fully mature entropy spot like the one shown in Fig. J.2 (b) and (c), a energy source term $\mathcal{Q}_{E}$ is used. The entropy spot is generated by means of local energy injection around a point, which is advected with the base flow. The energy source $\mathcal{Q}_{E}$ is a function of the the distance $r$ from the source center which moves with the fluid. This function is defined as follows

$$
\mathcal{Q}_{E}=\left\{\begin{array}{l}
\frac{\mathcal{A}_{E}}{2}\left(1+\cos \left(\pi \frac{r}{R_{s}}\right)\right) \text { if } r \in\left[0, R_{s}\right] \\
0 \forall r \notin\left[0, R_{s}\right]
\end{array}\right.
$$

where $r \geq 0$ by definition. The entropy-spot-core radius $R_{s}$ is set by the user. 
The amplitude $\mathcal{A}_{E}$ varies as a function of time

$$
\frac{2 \mathcal{A}_{E}}{\mathcal{A}_{\max }}=\left\{\begin{array}{l}
1-\cos \left(\frac{\pi t}{\tau_{\text {start }}}\right) \text { if } t \in\left[0, \tau_{\text {start }}\right] \\
2 \text { if } t \in\left(\tau_{\text {start }}, \tau_{\text {start }}+\tau_{\max }\right] \\
1+\cos \left(\frac{\pi\left(t-\left(\tau_{\text {start }}+\tau_{\max }\right)\right)}{\tau_{\text {end }}}\right) \text { if } t \in\left(\tau_{\text {start }}+\tau_{\max }, \tau_{\text {start }}+\tau_{\text {max }}+\tau_{\text {end }}\right] \\
0 \text { for } t \in\left(\tau_{\text {start }}+\tau_{\max }+\tau_{\text {end }}, t_{\text {end }}\right)
\end{array}\right.
$$

where the maximum amplitude $\mathcal{A}_{\text {max }}$, the startup time $\tau_{\text {start }}$ of generation, the amount of time $\tau_{\max }$ at which the generation is done with the maximum global amplitude, the time $\tau_{\text {end }}$ during which the generation process is ramped down are chosen by the user, $t=t_{\text {end }}$ is the time at which the simulation ends. I.e. entropy spot generation comprises three phases of duration $\tau_{\text {start }}, \tau_{\max }$ and $\tau_{\text {end }}$.

In Fig. J.3, the normalized density distribution on the generation block is shown at different times, along a line at $y=0.4 S_{1}$. For the case shown in Fig. J.3 the upstream Mach number was set to $M=0.053$ and the upstream release height was $h=0.4 S_{1}$. The maximum generation amplitude was set to $\mathcal{A}_{\max }=10^{-2}$, the entropy-spot-core radius $R_{s}=0.3 S_{1}, \tau_{\text {start }} U / S_{1}=0.32, \tau_{\max } U / S_{1}=0.16$ and $\tau_{\text {end }} U / S_{1}=0.32$. The resulting spot has a maximum density deviation ${ }^{1}$ of $(\delta \rho / \rho)_{\max }=-0.03$.

The shape of the entropy spot is shown plotted along a line which cuts trough the spot in Fig. J.4, for various entropy-spot-core radii. The relative density profiles shown in Fig. J.4, are for entropy spots which are close to leaving the generation block. One observes, that the entropy-spot-core radius $R_{s}$ set is in good approximation preserved, particularly for $R_{s}=0.3$ and 0.4 . The maximum density deviation, within two significant digits, is for all three radii $(\delta \rho / \rho)_{\max }=-0.03$.

The shape of the the entropy spot, generated with entropy-spot-core radius $R_{s}=0.3$ and upstream Mach numbers $M=0.053,0.097$ and 0.197 is shown in Fig. J.5. In Fig. J.5 the density deviation $\delta \rho$ with respect to its maximum density deviation $(\delta \rho)_{\max }$, is shown around the the peak. One observes that the relative shape is in good approximation the same for all three upstream Mach numbers, and that the initially imposed entropy-spot-core-radius is to a satisfactory degree maintained.

\footnotetext{
${ }^{1}$ In the main text (Chapter 6 ) the notation $\delta \rho / \rho$ is used for the maximum density deviation of the entropy spot. Here $(\delta \rho / \rho)_{\max }$ is used to differentiate with a density distribution $\delta \rho / \rho$, which in the main text is not allude to.
} 


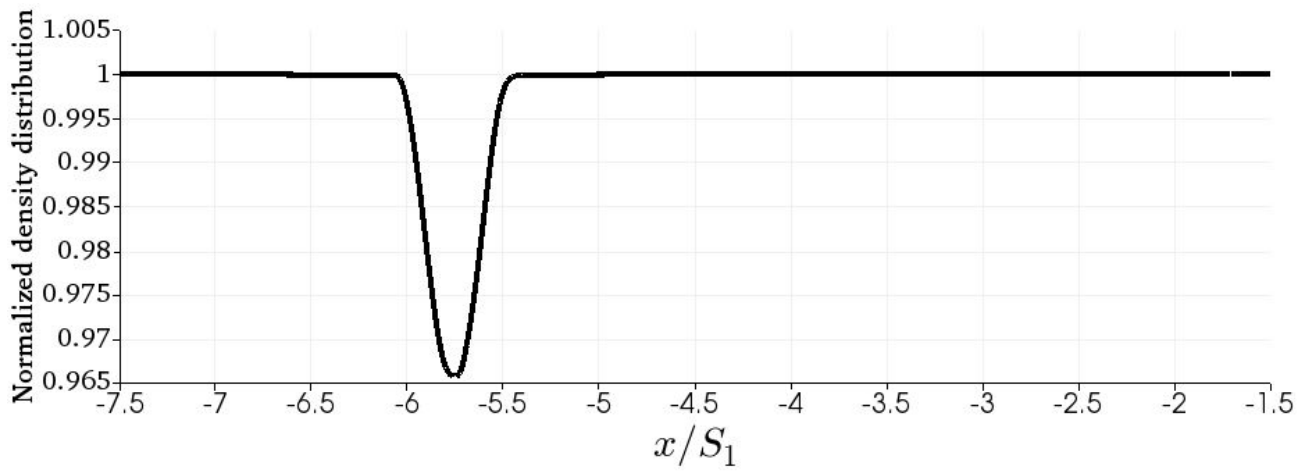

(a) $t U / S_{1} \simeq 0.64$ during the generation phase

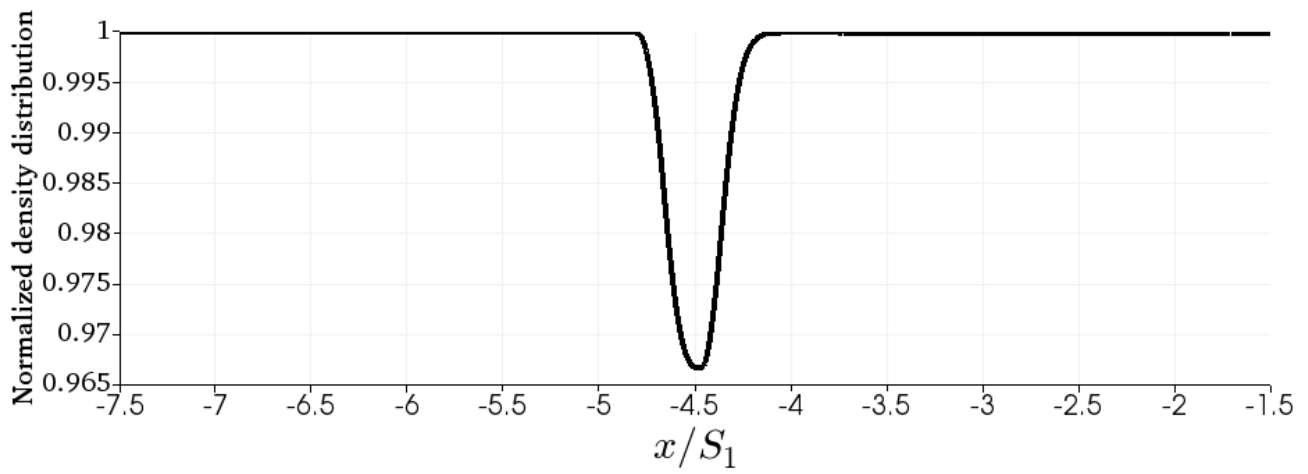

(b) $t U / S_{1} \simeq 1.9$ after generation phase

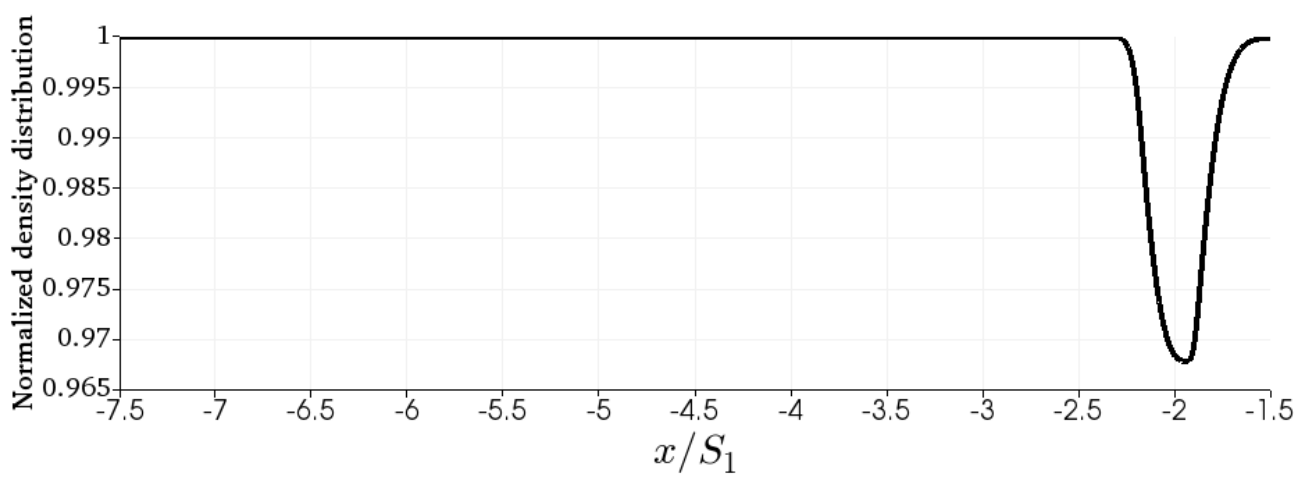

(c) $t U / S_{1} \simeq 4.5$ after generation phase close to texting the generation block

Figure J.3: The normalized density distribution along a line at $y=0.4 S_{1}$ at different stages on entropy spot generation block. The maximum density deviation of the spot in this is $(\delta \rho / \rho)_{\max }=-0.03$. The upstream Mach number during for this case was $M=0.053$. 


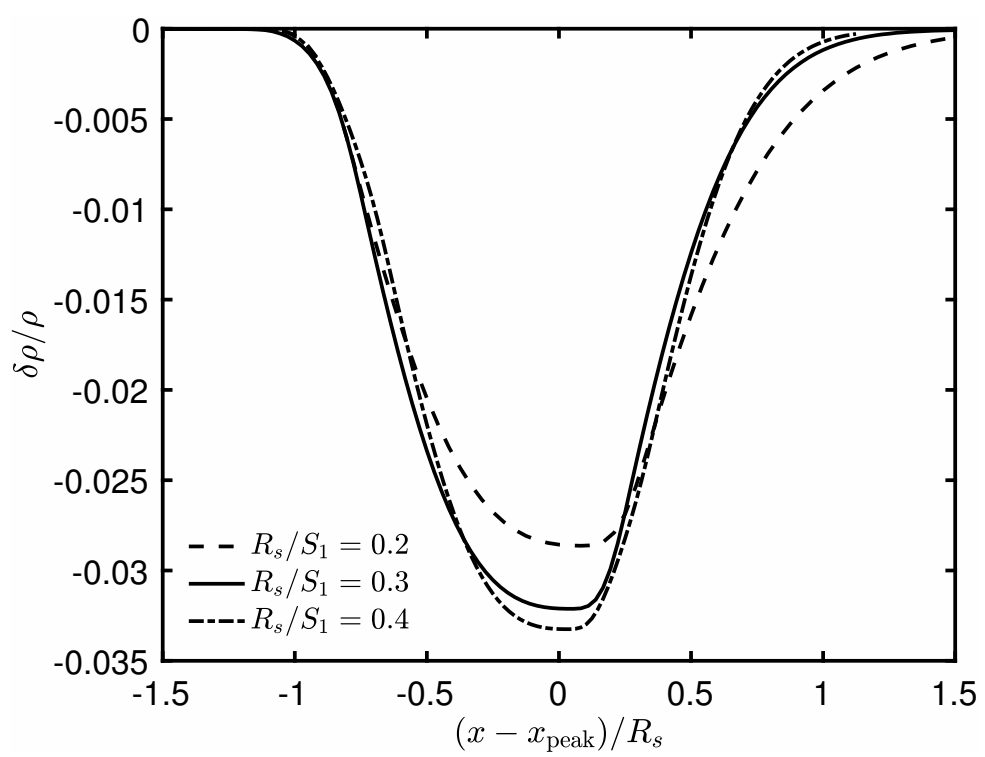

Figure J.4: Relative density profile $\delta \rho / \rho$ as a function of dimensionless position $\left(x-x_{\text {peak }}\right) / R_{s}$ relative to the peak along a line through the spot, for different core radii.

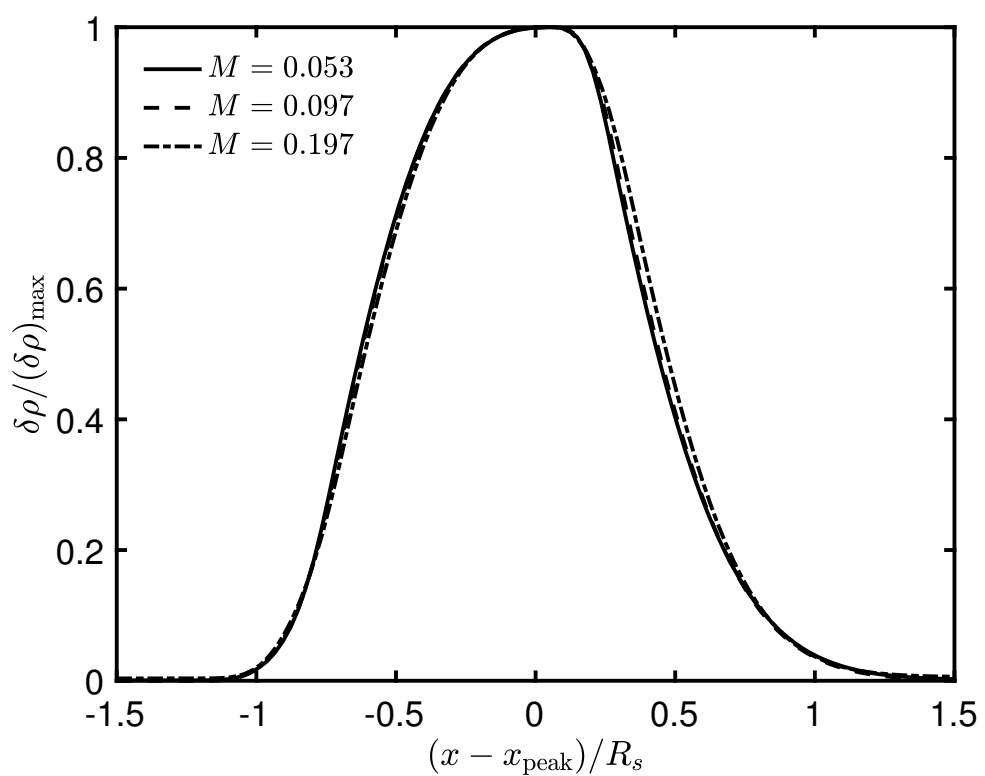

Figure J.5: Dimensionless shape of entropy spot, with entropy-spot-core radius $R_{s}=$ $0.3, \delta \rho /(\delta \rho)_{\max }$ as a function of dimensionless position $\left(x-x_{\text {peak }}\right) / R_{s}$, for different upstream Mach numbers. 



\section{Appendix K}

\section{List of publications}

\section{K.1 Peer reviewed conference proceedings}

CFA L. Hirschberg, C. Schram, and A. Hirschberg, "Self-sustained vortex shedding in a cold-gas scaled model of a large solid rocket motor", Congrès Français d'Acoustique in Le Mans, 2016

AIAA/CEAS L. Hirschberg, C. Schram, and A. Hirschberg, "Prediction of pulsations in a cold-gas scale-model of a solid rocket motor", 22nd AIAA/CEAS in Lyon, 2016

AIAA/CEAS L. Hirschberg, T. Schuller, C. Schram, J. Collinet, M. Yiao, and A. Hirschberg, "Interaction of a vortex with a contraction in a 2-dimensional channel: incompressible flow prediction of sound pulse", 23rd AIAA/CEAS in Denver, 2017

AIAA/CEAS L. Hirschberg, T. Schuller, C. Schram and J. Collinet, "Lumped model for vortex sound in large solid rocket motors", 24th AIAA/CEAS in Atlanta, 2018

\section{K.2 Peer reviewed published journal articles}

JSV L. Hirschberg, T. Schuller, J. Collinet, C. Schram and A. Hirschberg, "Analytical model for the prediction of pulsations in a cold-gas scale-model of a Solid Rocket Motor". Journal of Sound and Vibration, 419:452-468, 2018

JASA-EL L. Hirschberg, S. J. Hulshoff, J. Collinet, C. Schram and T. Schuller, "Vortex nozzle interaction in solid rocket motors: a scaling law for upstream acoustic response", Journal of the Acoustical Society of America, 144: EL46-EL51, 2018 


\section{K.3 Manuscript submitted for peer review}

AIAA/CEAS L. Hirschberg, S. J. Hulshoff, T. Schuller, C. Schram, J. Collinet, "Numerical simulations based evidence of impingement free sound production during vortex-nozzle interaction in solid rocket motors", 25th AIAA/CEAS in Delft, 2018

\section{K.4 Manuscript being prepared}

L. Hirschberg, S. J. Hulshoff, J. Collinet, C. Schram, T. Schuller, "Influence of nozzle cavity on indirect vortex and entropy sound production in solid rocket combustion chambers" 


\section{Appendix L}

\section{Résumé en Français}

Les moteurs de fusées à ergols solides (SRMs) sont sensibles aux instabilités hydrodynamiques qui peuvent déclencher des oscillations auto-entretenues de pression de grandes amplitudes lorsqu'elles se couplent à l'un des modes acoustiques du système. Le moteur de ces instabilités est la formation de structures tourbillonnaires cohérentes synchronisées par des ondes acoustiques longitudinales. Pour certaines conditions de fonctionnement, les ondes acoustiques générées par l'interaction de ces tourbillons avec la tuyère amorcée du moteur renforcent l'oscillation acoustique. La plupart des travaux précédents sur ce sujet traitent du seuil de déclenchement de ces instabilités à partir d'approches linéaires. L'objectif des travaux menés dans cette thèse est de déterminer l'amplitude et la fréquence des oscillations de pression au cycle limite des instabilités. Celui-ci est atteint par saturation non linéaire des sources, qui est la conséquence de la formation de grosses structures cohérentes. Dans ce cas l'interaction tourbillon tuyère devient insensible à l'amplitude de l'onde du mode acoustique établi dans le foyer. Dans ces conditions, on peut se concentrer sur l'interaction d'un tourbillon avec la tuyère dans le mécanisme de production sonore. En considérant un écoulement incompressible et l'absence de frottement, un premier modèle analytique est développé permettant de déterminer la production sonore d'un tourbillon ingéré par une tuyère bidimensionnelle plane, lorsque le tourbillon est traité comme une ligne vorticité. Des expériences précédentes indiquent que le volume de la cavité autour de l'entrée d'une tuyère intégrée a une grande influence sur l'amplitude des oscillations de pression dans les grands SRMs. On montre que ceci est dû au champ de vitesse acoustique induit par la compressibilité du gaz dans la cavité qui produit une fluctuation de vitesse transverse à la trajectoire du tourbillon. Une seconde alternative au modèle analytique incompressible est développée en considérant toujours l'absence de frottement, mais un modèle compressible de l'interaction tourbillon-tuyère. Celui-ci repose sur un code aéroacoustique pour les écoulements internes basé sur les équations d'Euler (EIA) qui est utilisé ici pour la simulation de l'interaction tourbillon-tuyère. Une étude systéma- 
tique de cette interaction a été menée pour une tuyère amorcée en variant le nombre de Mach de l'écoulement à l'approche de la tuyère, le rayon du coeur visqueux, le point amont d'où le tourbillon est lâché et la circulation du tourbillon. Les résultats ont permis de proposer un modèle de sources localisées pour des ondes planes basé sur une analyse théorique des lois d'échelles de ces phénomènes. Ce modèle apporte une compréhension physique et permet de prédire l'amplitude du pulse acoustique dû à l'interaction tourbillon-tuyère avec une erreur de moins de $25 \%$. Les simulations de ces interactions tourbillonstuyères ont été réalisées pour différents types de tuyères intégrées avec cavité ou sans cavité. Le code EIA est aussi utilisé pour explorer le potentiel d'une tuyère avec une modification graduelle de l'entrée pour réduire le bruit. Ce type de géométrie permettrait de réduire l'amplitude des oscillations de pression du cycle limite. Les travaux précédents font souvent référence au son produit par l'impact des tourbillons avec la paroi à l'entrée de la tuyère. Il est parfois fait référence à une annihilation du tourbillon. Les résultats obtenus dans le cadre des simulations Euler montrent que la production sonore a principalement lieu à l'approche de l'entrée de la tuyère, longtemps avant l'ingestion du tourbillon et sans collision avec la paroi. Dans le modèle analytique incompressible, l'effet de la cavité est représenté par une ligne de sources acoustiques. En employant un bilan énergétique, un modèle avec un seul paramètre de contrôle est formulé, qui permet de reproduire qualitativement le comportement du cycle limite d'oscillations de pression observées dans des expériences réalisées avec des gaz froids décrites dans la littérature. Finalement le modèle Euler est utilisé pour comparer la production de son par interaction tourbillon-tuyère avec celle due à l'ingestion d'une onde d'entropie, appelée aussi tache d'entropie. Contrairement au cas des tourbillons, le bruit produit par ingestion de taches d'entropie n'est pas sensible au volume de la cavité d'une tuyère intégrée. Ces résultats indiquent que le bruit produit par les tourbillons est dominant dans le cas des SRMs étudiés. L'ensemble de ces travaux permet d'améliorer la compréhension des phénomènes d'interaction entre des non-homogénéités de l'écoulement et la tuyère. Elle permet surtout de déterminer quels sont les facteurs de l'écoulement et les éléments géométriques importants qui pilotent le niveau sonore produit par ces interactions. Les modèles développés dans ces travaux, avec divers degrés d'approximation et de complexité permettent d'enrichir la gamme des outils de conception des SRMs. 


\section{References}

Achenbach, E. and E. Heinecke (1981). On vortex shedding from smooth and rough cylinders in the range of reynolds numbers $6 \times 10^{3}$ to $5 \times 10^{6}$. Journal of Fluid Mechanics 10(9), 239-251. (p. 6, 7)

Anthoine, J. (2000). Experimental and numerical study of aeroacoustic phenomena in large solid propellant boosters, with application to the Ariane 5 solid rocket motor. $\mathrm{Ph}$. D. thesis, Université Libre de Bruxelles, Belgium. (p. xii, 1, 2, 3, 4, 5, 7, 8, 11, 12, 13, 14, 15, 23, 36, 54, 55, 56, 57, 58, $60,66,110,111,112,113,114,115,126,127,128,129,134,136,138$, 139, 149, 152, 154, 155, 166)

Anthoine, J. (2014). Solid Propellant Pressure Oscillations. STO-EN-AVT206. RTO/NATO. (p. 4, 8, 13, 17, 18, 110)

Anthoine, J., J.-M. Buchlin, and J.-F. Guery (2003). Effect of nozzle cavity on resonance in large srm: Numerical simulations. Journal of Propulsion and Power 19(3), 374-384. (p. 5, 111)

Anthoine, J., J.-M. Buchlin, and A. Hirschberg (2002). Effect of nozzle cavity on resonance in large srm: Theoretical modeling. Journal of Propulsion and Power 18(2), 304-311. (p. 111, 113, 138, 148, 149)

Anthoine, J., P. Jézéquel, M. Prévost, and P. Prévot (2015). Variable fuel grain burn velocities to reduce solid-rocket-motor pressure oscillations. Journal of Propulsion and Power 31(1), 342-351. (p. 5, 7, 13, 14)

Anthoine, J., P. Jézéquel, M. Prévost, P. Prévot, and G. Casalis (2015). Variable fuel grains burn velocities to reduce solid-rocket-motor pressure oscillations. Journal of Propulsion and Power 31(1), 342-351. (p. 23)

Anthoine, J. and M. R. Lema (2009). Passive Control of Pressure Oscillations in Solid Rocket Motors: Cold-Flow Experiments. Journal of Propulsion and Power 25(3), 792-800. (p. 8, 112, 126)

Bake, F., N. Kings, A. Fischer, and I. Roehle (2009). Indirect combustion noise: Investigations of noise generated by the acceleration of flow inhomogeneities. Acta Acustica united with Acustica 95(3), 461-469. (p. 16)

Bake, F., C. Richter, B. Mühlbauer, N. Kings, I. Röhle, F. Thiele, and B. Noll (2009). The Entropy Wave Generator (EWG): A reference case on entropy noise. Journal of Sound and Vibration 326(3-5), 574-598. (p. 5, 9, 16)

Baum, J. D. and J. N. Levine (1986). Modeling of nonlinear longitudinal instability in solid rocket motors. Acta Astronautica 13(6-7), 339-348. 
(p. 11, 156)

Boyer, G., G. Casalis, and J.-L. Estivalèzes (2013). Stability analysis and numerical simulation of simplified solid rocket motors. Physics of Fluids 25(8), 084109. (p. 17)

Boyer, G., G. Casalis, and J.-L. Estivalèzes (2013). Stability and sensitivity analysis in a simplified solid rocket motor flow. Journal of Fluid Mechanics 722, 618-644. (p. 17)

Brown, J. W. and R. V. Churchill (2009). Complex Variables and Aplications (8th ed.). McGraw-Hill. (p. 39)

Brown, R. S., R. Dunlap, S. Young, and R. Waugh (1981). Vortex shedding as a source of acoustic energy in segmented solid rockets. Journal of Spacecraft 18(4), 312-319. (p. 110)

Bruggeman, J. C. (1987). Flow induced pulsations in pipe systems. Ph. D. thesis, Techinische Universiteit Eindhoven. (p. 154)

Bruggeman, J. C., A. Hirschberg, M. van Dongen, and A. Wijnands (1991). Self-sustained aero-acoustic pulsations in gas transport systems: Experimental study of the influence of closed side branches. Journal of Sound and Vibration 150(3), 371-393. (p. 6, 10, 12, 21, 29, 30, 62, 116, 117, $126,130,132,154)$

Buffum, F. G., G. L. Dehority, and R. O. S. E. W. Proce (1966). Acoustic losses of a subscale, cold-flow rocket motor for various " $\mathrm{j}$ " values. NAWEPS report 8971, NCTS TP 3932, US naval ordinace test station, China lake, CA, USA. (p. 28, 34)

Carmincino, C. (2009). Acoustics, vortex shedding, and low-frequency dynamics interaction in unstable hybrid rocket. Journal of Propulsion and Power 25, 1322-1335. (p. 26)

Casalis, G., G. Avalon, and J.-P. Pineau (1998). Spatial instability of planar channel flow with fluid injection through porous walls. Physics of Fluids 10(10), 2558-2568. (p. 9, 17)

Casalis, G. and F. Vuillot (2004). Motor flow instabilities - part 2: intrinsic linear stability of the flow induced wall injection. In Internal aerodynamics in solid rocket propulsion RTO-EN-023. NATO-RTO. (p. 13, 17, 54, 110)

Cerqueira, S. and D. Sipp (2014). Eigenvalue sensitivity, singular values and discrete frequency selection mechanism in noise amplifiers: the case of flow induced by radial wall injection. Journal of Fluid Mechanics 75\%, 770-799. (p. 9)

Chedevergne, F. and G. Casalis (2006, July). Detailed analysis of the thrust oscillations in reduced scale solid rocket motors. In 42nd AIAA/ASME/SAE/ASEE Joint Propulsion Conference and Exhibit, Sacramento. AIAA. (p. 17, 18, 19, 20)

Chedevergne, F., G. Casalis, and T. Féraille (2006). Biglobal linear stability analysis of the flow induced by wall injection. Physics of Fluids 18, 014103. (p. 17, 18)

Chedevergne, F., G. Casalis, and J. Majdalani (2012). Direct numerical sim- 
ulation and biglobal stability investigations of the gaseous motion in solid rocket motors. Journal of Fluid Mechanics 706, 190-218. (p. 17)

Clements, R. R. (1973). An inviscid model for two-dimensional vortex shedding. Journal of Fluid Mechanics 57, 321-336. (p. 41, 174, 175)

Colonius, T. and S. K. Lele (2004). Computational aeroacoustics: progress on nonlinear problems ofsoundgeneration. Progress in Aerospace Science 40, 345-416. (p. 9, 189)

Crighton, D. G. (1972). Radiation from vortex filament motion near a half plane. Journal of Fluid Mechanics 51, 357-362. (p. 37, 42, 176, 177)

Crighton, D. G., A. P. Dowling, J. E. F. Williams, M. Heckl, and F. G. Leppington (1992). Modern methods in analytical acoustics. Springer-Verlag. (p. 23)

Culick, F. E. C. (2004). Combustion instabilities in solid propellant rocket motors. In Internal aerodynamics in solid rocket propulsion RTO-EN-023. RTO/NATO. (p. 22, 110, 118, 119)

Culick, F. E. C. (2006). Unsteady Motions in Combustion Chambers for Propulsion Systems, Volume RTO-AG-AVT-039 of RTO AGARDograph. RTO/NATO. (p. 5, 9, 11, 12, 22, 28, 34, 110, 111, 125, 136, 156)

Curle, N. (1953). Influence of solid boundaries upon aerodynamic sound. Proceedings of the Royal Society Series A-Mathematical and Physical Sciences 216, 412-424. (p. 16)

Doisneau, F. (2013). Eulerian modeling and simulation of polydisperse moderately dense coalescing spray ows with nanometric-to-inertial droplets: application to Solid Rocket Motors. Ph. D. thesis, Ecole Centrale Paris. (p. 3)

Dotson, K., S. Koshigoe, and K. Pace (1997). Vortex shedding in a large solid rocket motor without inhibitors at the segmented interfaces. Journal of Propulsion and Power 13(2), 197-206. (p. 5, 11, 13, 14, 17, 23, 110, 111, 112, 114)

Dowling, A. P. and Y. Mahmoudi (2015). Combustion noise. Proceedings of the Combustion Institute 35, 65-100. (p. 5, 154)

Dupays, J. (2002). Two-phase unsteady flow in solid rocket motors Ecoulement diphasique instationnaire dans les moteurs à propergol solide. Aerospace Science and Technology 6, 413-422. (p. 5)

Durrieu, P., G. Hofmans, G. Ajello, R. J. J. Boot, Y. Auregan, and A. Hirschberg (2001). Quasisteady aero-acoustic response of orifices. Journal of Acoustical Society of America 110(2), 1859-1872. (p. 111)

Fabignon, Y., J. Anthoine, D. Davidenko, R. Devillers, J. Dupays, D. Gueyffier, Hijlkema, N. Lupoglazoff, J. M. Lamnet, L. Tessé, A. Guy, and C. Erades (2016, June). Recent advances in research on solid rocket propulsion. Journal AerospaceLab 11, 1-15. (p. 6, 8, 12, 13, 21, 110)

Fabignon, Y., J. Dupays, G. Avalon, F. Vuillot, N. Lupoglazoff, G. Casalis, and M. Prévost (2003). Instabilities and pressure oscillations in solid rocket motors. Aerospace Science and Technology 7(3), 191-200. (p. 4, 
8, 12, 13, 15, 23, 111, 155)

Ferretti, V. (2011). Numerical Simulations of Acoustic Resonance of Solid Rocket Motor. Ph. D. thesis, La Sapienza, University of Rome. (p. 11, 156)

Ferretti, V., B. Favini, E. Cavallini, F. Serraglia, and M. D. Giacinto (2011). Vortex-sound generated pressure oscillations simulation in internal flow by means of q-1d model. In 4 th European conference for aerospace sciences. (p. 11, 156)

Ffowcs Williams, J. E. and M. S. Howe (1975). The generation of sound by density inhomogeneities in low Mach number nozzle flows. Journal of Fluid Mechanics 70(03), 605. (p. 9, 16, 22, 138, 140)

Fischbach, S. R. and J. Majdalani (2009). Volume-to-surface reduction of vorticoacoustic stability integrals. Journal of Sound and Vibration 321(35), 1007-1025. (p. 110)

Flandro, G. A. (2003). Aeroacoustic Instability in Rockets. AIAA Journal 41(3), 485-497. (p. 110)

Flandro, G. A., S. R. Fischbach, and J. Majdalani (2007). Nonlinear rocket motor stability prediction: Limit amplitude, triggering, and mean pressure shift. Physics of Fluids 19(9), 094101. (p. 11, 110, 151)

Gallier, S. and F. Godfroy (2009). Aluminum Combustion Driven Instabilities in Solid Rocket Motors. Journal of Propulsion and Power 25 (2), 509-521. (p. 5, 9, 17, 26)

Gallier, S., M. Prevost, J. Hijlkema, , and M. Roumy (2009). Effects of cavity on thurst oscillations in subscale solid rocket motors. In 45th AIAA/ASME/ASEE Joint Propulsion Conference \& Exhibit, Number 2009-5253. AIAA. (p. xiii, 5, 6, 8, 13, 21, 23, 36, 54, 60, 111, 117, $118,130,135,138,139,147,148,149,152,154,155)$

Gazanion, B., F. Chedevergne, and G. Casalis (2013). On the laminarturbulent transition in injection-driven porous chambers. Experiments in Fluids 55(1). (p. 8, 9)

Genot, A., S. Gallier, and T. Schuller (2017). A numerical analysis of the aluminium combustion driven instability in solid rocket motors. In EUCASS Paper EUCASS2017-064. EUCASS. (p. 5, 9, 17, 60)

Genot, A., S. Gallier, and T. Schuller (2018). Thermo-acoustic instabilities driven by fuel droplet lifetime oscillations. In Proceedings of the Combustion Institute. (p. 5, 9, 17)

Gilbarg, D. (1960). Handbuch der Physik: Strömungsmechanik III, Volume IX, Chapter Jets and Cavities. Berlin, Germany: Springer-Verlag. (p. 115, 184)

Golliard, J., S. Belfroid, A. Hirschberg, and G. Nakiboglu (2013). U-Rans model for the prediction of the acoustic sound power generated in a whistling corrugated pipe. In ASME 2013 Pressure Vessels and Piping Conference, pp. 1-6. (p. 117)

Greatrix, D. R. (2011). Scale Effects on Solid Rocket Combustion Instability 
Behaviour. Energies 4(1), 90-107. (p. 11, 110, 151, 156)

Griffond, J. and G. Casalis (2000). On the dependence on the formulation of some nonparallel stability approaches applied to the Taylor flow. Physics of Fluids 12(2), 466-468. (p. 17, 166)

Griffond, J. and G. Casalis (2001). On the nonparallel stability of the injection induced two-dimensional Taylor flow. Physics of Fluids 13(6), 1635-1644. (p. 17)

Griffond, J., G. Casalis, and J. P. Pineau (2000). Spatial instability of flow in a semiinfinite cylinder with fluid injection through its porous walls. European Journal of Mechanics, B/Fluids 19(1), 69-87. (p. 17)

Guery, J., F. Godfroy, S. Ballereau, S. Gallier, P. Della Pieta, O. Orlandi, E. Robert, and N. Cesco (2008, 2018/09/29). Thrust Oscillations in Solid Rocket Motors. American Institute of Aeronautics and Astronautics. (p. 17)

Hart, R. W. and F. T. McClure (1959). Combustion instability: Acoustic interaction with a burning surface. Journal of Chemical Physics 30, 15011414. (p. 9)

Henrici, P. (1974). Applied and Computational Complex Analysis, Volume I. NY, USA: Wiley-Interscience. (p. 36, 39, 60, 69, 122, 167, 170, 174)

Hijlkema, J., M. Prevost, and G. Casalis (2011). On the importance of reduced scale ariane 5 p230 solid rocket motor models in the comprehension and prevention of thrust oscillations. G. CEAS Space J 1(99). (p. 5, 6, $8,17)$

Hirschberg, L., S. J. Hulshoff, J. Collinet, C. Schram, and T. Schuller (2018, July). Vortex nozzle interaction in solid rocket motors: A scaling law for upstream acoustic response. The Journal of the Acoustical Society of America 144(1), EL46-EL51. (p. 139)

Hirschberg, L., C. Schram, and A. Hirschberg (2016). Prediction of pulsations in a cold-gas scale-model of a solid rocket motor. In 22nd AIAA/CEAS in Lyon. (p. 22, 112, 116, 120, 183)

Hirschberg, L., T. Schuller, J. Collinet, C. Schram, and A. Hirschberg (2018). Analytical model for the prediction of pulsations in a cold-gas scale-model of a solid rocket motor. Journal of Sound and Vibration 419, 452-468. (p. 22, 54, 57, 60, 106, 138, 139, 148, 149, 154)

Hirschberg, L., T. Schuller, C. Schram, J. Collinet, M. Yiao, and A. Hirschberg (2017). Interaction of a vortex with a contraction in a 2-dimensional channel: incompressible flow prediction of sound pulse. In 23rd AIAA/CEAS Aeroacoustics conference. (p. 54, 60, 88, 99, 122, 138)

Hofmans, G. C. J., R. J. J. Boot, P. P. J. M. Durrieu, Y. Auregan, and A. Hirschberg (2001). Aeroacoustic response of a slit-shaped diaphragm in a pipe at low helmholtz number, 1: quasi-steady results. Journal of Sound and Vibration 244(1), 35-56. (p. 115)

Howe, M. (1980). The dissipation of sound at an edge. Journal of Sound and 
Vibration 70(3), 407-411. (p. 120)

Howe, M. S. (1975). Contributions to the theory of aerodynamic sound, with application to excess jet noise and the theory og the flute. Journal of Fluid Mechanics 71, 625-673. (p. 45, 138, 146, 148)

Howe, M. S. (1984). On the Absorption of Sound by Turbulence and Other Hydrodynamic Flows. IMA Journal of Applied Mathematics 32(1-3), 187-209. (p. 12, 156)

Howe, M. S. (1998). Acoustics of fluid-structure interactions. Cambridge, UK: Cambridge university press. (p. 28, 42, 118, 119, 136, 151, 153)

Howe, M. S. (2007). Hydrodynamics and sound. Cambridge, UK: Cambridge university press. (p. 136)

Howe, M. S. (2014). Acoustics and Aerodynamic Sound. UK: Cambridge University Press. (p. 16, 22, 23, 36, 37, 41, 42, 45, 61, 62, 152, 175)

Hulshoff, S. J. (2016a). Computational modelling: lecture notes. TU Delft. (p. 8, 69, 179)

Hulshoff, S. J. (2016b, October). EIA an Euler Code for Internal Aeroacoustics: method description and user's guide. Delft, the Netherlands: Faculty of Aerospace Engineering, Delft University of Technology. (p. xiii, xiv, 69, 70, 72, 139, 187, 188, 189, 190, 191, 192, 193, 194, 195, 196, 199)

Hulshoff, S. J. (2016c, July). Private comunication. (p. 47)

Hulshoff, S. J. (2018, February 15th). Private communication. During a visit at TU Delft. (p. 68, 69)

Hulshoff, S. J., A. Hirschberg, and G. C. J. Hofmans (2001). Sound production of vortex nozzle interaction. Journal of Fluid Mechanics 439, 335-352. (p. 9, 36, 45, 47, 49, 50, 55, 60, 130, 138, 139, 152)

Kings, N. (2014). Indirect combustion noise: Experimental investigation of the vortex sound generation in nozzle flows. Ph. D. thesis, Technische Universität Berlin. (p. 5, 9)

Kings, N. and F. Bake (2010). Indirect combustion noise: noise generation by accelerated vorticity in a nozzle flow. International Journal of Spray and Combustion Dynamics 2(3), 253-266. (p. 5, 9)

Kuentzmann, P., V. Yang, J. W. Murdock, W. A. Johnston, G. Lengelle, J. Duterque, J. F. Trubert, M. W. Beckstead, M. Salita, F. Vuillot, J. Guery, and F. E. C. Culick (2004). Internal Aerodynamics in Solid Rocket Propulsion. RTO EDUCATIONAL NOTES EN-023 AVT-096. RTO NATO. (p. 5, 8, 9, 12, 21, 111)

Kundu, P. K. (1990). Fluid Mechanics. Academic Press, Inc. (p. 6)

Laboureur, D., B. Tóth, and J. Anthoine (2010). Investigation of the TaylorCulick Flow Through Particle Image Velocimetry and Numerical Simulation. AIAA Journal 48(6), 1077-1084. (p. 110)

Lacassagne, L. (2017). Simulations et analyses de stabilité linéaire du d'etachement tourbillonnaire d'angle dans les moteurs á propergol solide. $\mathrm{Ph}$. D. thesis, INP Toulouse. (p. 9, 60)

Landau, L. D. and E. M. Lifshitz (1987). Fluid Mechanics (2nd ed.). Elsevier 
Science Ltd. (p. 6)

Levine, J. A. Y. N. and J. D. Baum (1982). Modeling of nonlinear combustion instability in solid propellantrocket motors. In Nineteenth Symposium on Combustion/The Combustion Institute, pp. 769-776. (p. 11, 156)

Leyko, M., S. Moreau, F. Nicoud, and T. Poinsot (2011). Numerical and analytical modelling of entropy noise in a supersonic nozzle with a shock. Journal of Sound and Vibration 330(16), 3944-3958. (p. 9)

Majdalani, J., S. R. Fischbach, and G. A. Flandro (2006). Improved energy normalization function in rocket motor stability calculations. Aerospace Science and Technology 10(6), 495-500. (p. 22)

Marble, F. and S. Candel (1977, November). Acoustic disturbance from gas non-uniformities convected through a nozzle. Journal of Sound and Vibration 55(2), 225-243. (p. 9, 33, 46, 61, 65, 111, 138, 156, 183)

Michalke, A. (1965). On spatially growing disturbances in an inviscid shear layer. Journal of Fluid Mechanics 23, 521-544. (p. 117)

Milne-Thomson, L. M. (1968). Theoretical Hydrodynamics (5th ed.). London, UK: MacMillan Education. (p. 39, 167, 174)

Morfey, C. (1971). Sound transmission and generation in ducts with flow. Journal of Sound and Vibration 14(1), $37-55$. (p. 28)

Morgans, A. S. and I. Duran (2016). Entropy noise: a review of theory, progress and challenges. International Journal of Spray and Combustion Dynamics 8(4), 285-298. (p. 5, 138, 154)

Nelson, P., N. Halliwell, and P. Doak (1983). Fluid dynamics of a flow excited resonance, part ii; flow acoustic interaction. Journal of Sound and Vibration 91, 375-402. (p. 116, 154)

Nicoud, F. and K. Wieczorek (2009). About the zero Mach number assumption in the calculation of thermoacoustic instabilities. International Journal of Spray and Combustion Dynamics 1(1), 67-111. (p. 36)

Peters, M. C. A. M. (1993). Aeroacoustic sources in internal flows. Ph. D. thesis, Technische Universiteit Eindhoven, Eindhoven, NL. (p. 123)

Pierce, A. (1989). Acoustics: An introduction to its physical princi- pals and applications. Acoustical Society of America. (p. 11)

Piomelli, U., E. Balaras, P.-A. Fernando, P. Sagaut, and R. Verzicco (2018). Large Eddy Simulations. Theory and Applications. Von Karman Insitute for Fluid Dynamics. (p. 6, 21)

Powell, A. (1961). On the degetone. Journal of the Acoustical Society of America 216, 412-424. (p. 16)

Prandtl, L. (1904). über fl|'ussigkeitsbewegung bei sher kleiner reibung. Intern. Math. Kongr. Heidelberg 2, 484-491. (p. 9)

Prandtl, L. and O. G. Tiejens (1934). Fundamentals of Hydro- and Aeromechanics. NY: Dover Pub. (p. 5, 37)

Price, E. and J. Sofferis (1958). Combustion instability in solid propellant rocket motors. Jet Propulsion 28, 190-192. (p. 9)

Ribereau, D., T. Pevergne, P. Le Helley, L. Jacques, M. Prevost, and 
N. Cesco (2005, 2018/09/28). Thrust Oscillations Passive Control on Large Solid Rocket Motors. American Institute of Aeronautics and Astronautics. ( $p .7)$

Rienstra, S. W. and A. Hirschberg (2015). An Introduction to Acoustics (Revised ed.). Technische Universiteit Eindhoven. (p. 23)

Saffman, P. G. (1992). Vortex Dynamics. UK: Cambridge University Press. (p. 5, 72, 174)

Schlichting, H. (1979). Boundary layer theory (7th ed.). McGraw-Hill. (p. 9)

Schmidt, B. (2015). Compressible Flow Through Porous Media with Application to Injection. Technical report, California Institute of Technology. (p. 111, 185)

Stella, F. and F. Paglia (2009). Pressure oscillations in solid rocket motors: Effect of nozzle cavity. Aerotecnica 88(1/2), 30-41. (p. 21)

Stella, F. and F. Paglia (2011). Pressure oscillations in solid rocket motors: Numerical study. Aerospace Science and Technology 15(1), 53-59. (p. 8, 21)

Tao, W., T. Schuller, M. Huet, and R. F. (2017). Coherent entropy induced and acoustic noise separation in compact nozzles. Journal of Sound and Vibration 394, 237-255. (p. 9, 138)

Thompson, P. A. (1972). Compressible-Fluid Dynamics. NY, USA: MacGraw Hill. (p. 4, 10, 26, 56, 70, 121)

Tonon, D., A. Hirschberg, J. Golliard, and S. Ziada (2011). Aeroacoustics of pipe systems with closed branches. International Journal of Aeroacoustics 10(2-3), 201-276. (p. 20, 21, 63, 116, 118)

Tonon, D., J. Willems, and A. Hirschberg (2011). Self-sustained oscillations in pipe systems with multiple deep side branches: Prediction and reduction by detuning. Journal of Sound and Vibration 330, 5894-5912. (p. 117)

van Dyke, M. (1964). Perturbation methods in fluid mechanics. Academic Press, Inc. (p. 9)

Varapaev, V. N. and V. I. Yagodkin (1969). Flow stability in a channel with porous wall. Izv. AN SSSR. Mekhanika Zhidkosi i Gaza 4(5). (p. 17)

Venkatakrishnan, V. and A. Jameson (1988). Computation of unsteady transonic flows by the solution of euler equations. AIAA Journal 26(8), 974981. (p. 69, 72, 139)

Verge, M. P., A. Hirschberg, and R. Caussé (1997). Sound production in recorderlike instruments. ii. a simulation model. Journal of Acoustical Society of America 101, 2925. (p. 63)

Vervisch, L., D. Veynante, R. Koch, S. Richard, A. Dreizler, C. Hasse, and D. Haworth (2017). Lecture Series on Turbulent Combustion. Von Karman Insitute for Fluid Dynamics. (p. 5)

Vuillot, F. (1995). Vortex- shedding phenomena in solid rocket motors. Journal of Propulsion and Power 11(4), 626-639. (p. 5, 9, 12, 110)

Vuillot, F. and G. Casalis (2004). Motor flow instabilities - part 1. In Inter- 
nal aerodynamics in solid rocket propulsion RTO-EN-023. NATO-RTO. (p. 5, 13, 14, 15, 110)

Wang, M. and D. R. Kassoy (1992, 2018/10/04). Standing acoustic waves in a low mach number shear flow. AIAA Journal 30(7), 1708-1715. (p. 12, 28)

Waxing, S., L. Shipeng, Z. Qiao, L. Junwei, Y. Qingqing, and W. Ningfei (2013). Influence of thermal inhibitor position and temperature on vortexshedding-driven pressure oscillations. Chinese Journal of Aeronautics 26, 544-553. (p. 21)

Yildiz, D. (2000). Influence of radial injected flow on the aeroacoustic coupling in solid propellant boosters. Technical report, VKI, Rhode-StGenèse, Belgium. (p. 115, 126, 128, 136)

Zhang, Q., Z. Wei, W. Su, J. Li, and N. Wang (2012). Theoretical Modeling and Numerical Study for Thrust-Oscillation Characteristics in Solid Rocket Motors. Journal of Propulsion and Power 28(2), 312-322. (p. 21) 



\title{
universite் PARIS-SACLAY
}

\section{Titre : Modélisation des oscillations de pression auto-entretenues induites par des tourbillons dans les moteurs à propergol solide \\ Mots clés : Modélisation d'ordre reduit, Oscillations auto-entrenues, Bruit tourbillonnaire, Moteurs à propergol solide, Bruit indirect, Loi d'échelle}

Résumé : Les moteurs de fusées à ergols solides (SRMs) sont sensibles aux instabilités hydrodynamiques qui peuvent déclencher des oscillations autoentretenues de pression de grandes amplitudes lorsqu'elles se couplent à l'un des modes acoustiques du système. Le moteur de ces instabilités est la formation de structures tourbillonnaires cohérentes synchronisées par des ondes acoustiques longitudinales. Pour certaines conditions de fonctionnement, les ondes acoustiques générées par l'interaction de ces tourbillons avec la tuyère amorcée du moteur renforcent l'oscillation acoustique. La plupart des travaux précédents sur ce sujet traitent du seuil de déclenchement de ces instabilités à partir d'approches linéaires. L'objectif des travaux menés dans cette thèse est de déterminer l'amplitude et la fréquence des oscillations de pression au cycle limite des instabilités. Celui-ci est atteint par saturation non linéaire des sources, qui est la conséquence de la formation de grosses structures cohérentes. Dans ce cas l'interaction tourbillon tuyère devient insensible à l'amplitude de l'onde du mode acoustique établi dans le foyer. Dans ces conditions, on peut se concentrer sur l'interaction d'un tourbillon avec la tuyère dans le mécanisme de production sonore. Une étude systématique de cette interaction a été menée pour une tuyère amorcée. Les résultats ont permis de proposer un modèle de sources localisées pour des ondes planes basé sur une analyse théorique des lois d'échelles de ces phénomènes. Les simulations de ces interactions tourbillons-tuyères ont été réalisées pour différents types de tuyères intégrées avec cavité ou sans cavité. En employant un bilan énergétique, un modèle avec un seul paramètre de contrôle est formulé, qui permet de reproduire qualitativement le comportement du cycle limite d'oscillations de pression observées dans des expériences réalisées avec des gaz froids décrites dans la littérature. L'ensemble de ces travaux permet d'améliorer la compréhension des phénomènes d'interaction entre des non-homogénéités de l'écoulement et la tuyère. Elle permet surtout de déterminer quels sont les facteurs de l'écoulement et les éléments géométriques importants qui pilotent le niveau sonore produit par ces interactions.

Title : Low order modeling of vortex driven self-sustained pressure pulsations in solid rocket motors

Keywords : Reduced-order modeling, Vortex Sound, Solid Rocket Motor, Indirect sound, Scaling law, Sustained pressure oscillations

\begin{abstract}
Solid Rocket Motors (SRMs) can display self-sustained acoustic oscillations driven by coupling between hydrodynamic instabilities of the internal flow and longitudinal acoustic standing waves. The hydrodynamic instabilities are triggered by the acoustic standing wave and results in the formation of coherent vortical structures. For nominal ranges of flow conditions the sound waves generated by the interaction between these vortices and the choked nozzle at the end of the combustion chamber reinforces the acoustic oscillation. Most available literature on this subject focuses on the threshold of instability using a linear model. The focus of this work is on the prediction of the limit-cycle amplitude. The limit cycle is reached due to nonlinear saturation of the source, as a consequence of the formation of large coherent vortical structures. In this case the vortex-nozzle interaction becomes insensitive to the amplitude of the acoustic standing wave. Hence, one can focus on the sound generation of a vortex with the nozzle. A dedicated numerical simulation study focusing on ele-

mentary processes such as vortex-nozzle and entropy spot-nozzle interaction allows a systematic variation of relevant parameters and yields insight which would be difficult by means of limit cycle studies of the full engine. A systematic study of the vortex-nozzle interaction in the case of a choked nozzle has been undertaken by varying. The results are summarized by using a lumped element model for plane wave propagation. Simulations are made for integrated nozzles with a surrounding cavity and nozzle inlets mounted flush in a wall normal to the combustion chamber wall. Using an energy balance approach, a single fitparameter model is formulated which qualitatively predicts limit-cycle observations in cold gas-scale experiments reported in the literature. In addition to insight, this study provides a systematic procedure to develop a lumped element model for the sound source due to non-homogeneous flow-nozzle interactions in SRMs. Such lumped models based on experimental data or a limited number of flow simulations can be used to ease the design of SRMs.
\end{abstract}

\section{Université Paris-Saclay}

Espace Technologique / Immeuble Discovery

Route de l'Orme aux Merisiers RD 128 / 91190 Saint-Aubin, France 